\title{
A Dependable Anisotropic \\ Magnetoresistance Sensor System for Automotive Applications
}

\author{
Andreina Zambrano
}




\section{A DEPENDABLE ANISOTROPIC MAGNETORESISTANCE SENSOR SYSTEM FOR AUTOMOTIVE APPLICATIONS}

Andreina Claret Zambrano Costantini 
Members of the graduation committee:

Dr. ir.

H. G. Kerkhoff

Prof. dr. ir.

Prof. dr. ir.

Prof. dr. ir.

Prof. dr.

Prof. dr.

Dr.

Prof. dr.
G. J. M. Smit

P. J. M. Havinga

G. J. M. Krijnen

J. Figueras

J. Machado da Silva

K. Reimann

J. N. Kok
University of Twente (Supervisor)

University of Twente

University of Twente

University of Twente

Polytechnic University of Catalonia

University of Porto

NXP Semiconductors (Expert)

University of Twente (Chairman and secretary)

\section{UNIVERSITY OF TWENTE.}

Faculty of Electrical Engineering, Mathematics and Computer Science (EEMCS), Computer Architecture for Embedded Systems (CAES) group

Ph.D. Thesis Series No. 18-009

Institute on Digital Society

P.O. Box 217, $7500 \mathrm{AE}$

Enschede, The Netherlands

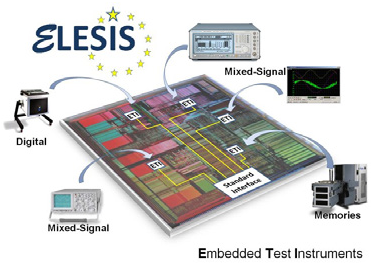

This research has been conducted within the ENIAC project European Library-based flow of Embedded Silicon and test Instruments (ELESIS) which is financially supported by the European Commission (EC) and the Netherlands Enterprise Agency (RVO)

Copyright (c) 2018 Andreina Zambrano, Enschede, The Netherlands.

All rights reserved. No part of this book may be reproduced or transmitted, in any form or by any means, electronic or mechanical, including photocopying, recording, or by any information storage or retrieval system, without prior written permission of the author.

This thesis was typeset using $\mathrm{AT}_{\mathrm{E}} \mathrm{X}$.

The cover page was designed by Ipskamp printing. Image from istockphoto.com.

This thesis was printed by Ipskamp printing, Enschede, The Netherlands.

ISBN 978-90-365-4599-0

ISSN 2589-7721; IDS Ph.D Thesis Series No. 18-009

DOI $\quad 10.3990 / 1.9789036545990$ 


\title{
A DEPENDABLE ANISOTROPIC MAGNETORESISTANCE SENSOR SYSTEM FOR AUTOMOTIVE APPLICATIONS
}

\author{
DISSERTATION \\ to obtain \\ the degree of doctor at the University of Twente \\ on the authority of the rector magnificus, \\ prof. dr. T.T.M. Palstra, \\ on account of the decision of the graduation committee, \\ to be publicly defended \\ on Wednesday 5th of September 2018 at 12:45
}

by

Andreina Claret Zambrano Costantini

born on 20th of February 1984, in Caracas, Venezuela 
This dissertation has been approved by:

Supervisor: Dr. ir. H. G. Kerkhoff

Copyright (c) 2018 Andreina Zambrano ISBN 978-90-365-4599-0 


\begin{abstract}
The increasing usage of electronic systems in automotive applications aims to enhance passenger safety as well as the performance of the cars. In modern vehicles mechanical and hydraulic systems traditionally used have been replaced by X-by-wire systems in which the functions are performed by electronic components. However, the components required should be reliable, have a high-performance, low-cost and capable of operating for a long time in a highly dependable manner despite the harsh operating conditions in automotive applications. Dependability represents the reliance that a user justifiably poses on the service offered by a system, being this especially important in safety-critical applications in which a failure can constitute a threat to people or the environment.
\end{abstract}

Magnetic sensors represent an excellent option to replace the wear-affected potentiometers which have been traditionally used. They offer several key advantages including mechanical robustness due to the non-contact measurement principle, extensive operating temperature range and low manufacturing cost. An Anisotropic Magnetoresistance (AMR) sensor is a type of magnetic sensor often used for angle measurements in applications such as steering or engine control which are considered to be safety-critical. The angle is obtained from two sinusoidal signals at the sensor outputs, which in theory should be perfect signals. However, in practice, they include undesired parameters such as offset voltage, amplitude imbalance and additional harmonics that affect the accuracy of the calculated angle and hence the performance of the sensor. Until now the offset voltage is the parameter mainly compensated for by calibration under factory conditions. Although the undesirable parameters drift over time, the sensor performance remains currently within the tolerance band permitted, and therefore aging compensation is usually not applied. However, this will change in the future because the tolerance band for drifting is expected to decrease as better performance of the sensor will be required over time, especially with the current trend of X-by-wire systems and autonomous cars.

In order to have a better understanding of the aging effects on AMR sensors because to the best of our knowledge there has been little published data on this issue, it was decided to perform a set of accelerated degradation tests. The results show that aging compensation will be required in the future, especially for the offset voltage that turns out to be the largest contributor to angle errors at the start of the sensor's life, but it also shows the most significant drift due to aging effects. In the second place the amplitude imbalance between the sinusoidal signals at the sensor outputs is causing angle errors and as last the harmonics.

Besides performance degradation, AMR sensors can also be affected by catastrophic faults that in principle cause the sensor to suddenly stop working. Therefore, the sensor dependability should be improved in order to guarantee that it will satisfy the continuous increasing dependability as well as accuracy requirements demanded by automotive applications. This research proposes an AMR sensor system that 
includes a fault-tolerant approach to handle catastrophic faults and self-X properties to maintain the performance of the sensor during its lifetime. Additionally, an interface with the IEEE 1687 standard has been considered, so the sensor is able to communicate with other components of the system in which it is integrated.

Self-X properties represent the capability of a system to perform certain functions on its own without any external help. In our system, self-monitoring of the maximum angle error is proposed to determine whether aging compensation is required in which case our self-calibration method allows updating the compensation factors for offset voltage as well as amplitude imbalance. Our fault-tolerant approach allows the sensor to continue operating although a catastrophic fault occurs in any of its magnetoresistances or the connections to the power supply or ground. All this is aimed to be executed online based on digital processing during the sensor lifetime. The system has been verified using data obtained from an analytical model of the sensor but also with data measured in commercial AMR sensors. The dependability assessment has been performed focussed on the dependability attributes, reliability, safety, maintainability and availability in order to verify the dependability improvement that can be obtained with the proposed system.

In conclusion, an AMR sensor system for angle measurements in automotive applications has been proposed which allows guaranteeing the correct service of the sensor despite unexpected failures or undesired aging effects. This is more than ever important with the current trend of reliance in electronic systems for the correct service of cars. 


\section{Samenvatting}

Het toenemende gebruik van elektronische systemen in de auto-industrie heeft ten doel de veiligheid van de passagiers evenals de prestaties van de auto's te verbeteren. In moderne voertuigen zijn de van oudsher gebruikte mechanische en hydraulische systemen vervangen door X-by-wire-systemen, waarbij de functies door elektronische componenten worden uitgevoerd. De vereiste componenten moeten echter bedrijfszeker zijn, hoge prestaties leveren, weinig kosten en in staat zijn om ondanks de zware bedrijfsomstandigheden in de auto langdurig op een zeer betrouwbare manier te functioneren. Betrouwbaarheid staat voor het vertrouwen dat een gebruiker terecht stelt in de door een systeem geboden service. Dit is vooral van belang bij veiligheidskritische toepassingen, waarbij een storing een bedreiging kan vormen voor mens of omgeving.

Magnetische sensoren vormen een uitstekende optie ter vervanging van de traditioneel toegepaste potentiometers, die aan slijtage onderhevig zijn. Zij bieden een aantal opvallende voordelen, waaronder mechanische robuustheid dankzij het contactloze meetprincipe, een groot omgevingstemperatuurbereik, en geringe productiekosten. De anisotrope magnetoresistentie (AMR)-sensor is een type magnetische sensor, dat vaak wordt ingezet voor hoekmetingen bij toepassingen die als veiligheidskritisch worden beschouwd, zoals de stuurinrichting of het motormanagement. De hoek wordt verkregen uit twee sinusvormige signalen op de sensoruitgangen, die in theorie perfecte signalen zouden moeten zijn. In de praktijk bevatten ze echter ongewenste parameters, zoals offsetvoltage, amplitude-onbalans en bijkomende harmonischen, die de nauwkeurigheid van de berekende hoek en dus de prestaties van de sensor aantasten. Tot nu toe wordt de parameter van het offsetvoltage hoofdzakelijk door kalibratie onder fabrieksomstandigheden gecompenseerd. Hoewel de ongewenste parameterwaarden in de loop van de tijd wel verlopen, blijven de sensorprestaties momenteel binnen het toegestane tolerantiebereik en wordt er daarom meestal geen verouderingscompensatie toegepast. Dit zal evenwel in de toekomst gaan veranderen. Naar verwachting zal het tolerantiebereik voor de verlopende waarden afnemen, omdat betere prestaties van de sensor vereist zullen worden, vooral in het kader van de huidige trend naar X-by-wire-systemen en autonome voertuigen.

Om beter inzicht te krijgen in de gevolgen van veroudering voor AMR-sensoren, omdat er naar ons beste weten maar weinig gepubliceerde gegevens over dit onderwerp bestaan, werd besloten een set versnelde degradatietesten uit te voeren. De resultaten tonen aan dat de verouderingscompensatie in de toekomst vereist zal zijn. Dit is vooral van toepassing voor het offsetvoltage, dat aan het begin van de sensorlevensduur de grootste oorzaak voor hoekfouten blijkt, maar dat ook het

grootste verloop ten gevolge van veroudering vertoont. In de tweede plaats veroorzaakt de amplitude-onbalans tussen de sinusvormige signalen op de sensoruitgangen harmonischen en hoekmeetfouten.

Behalve aan prestatievermindering kunnen AMR-sensoren ook onderhevig zijn 
aan catastrofale storingen, die er in principe voor zorgen dat de sensor plotseling uitvalt. Daarom moet de betrouwbaarheid van de sensor worden verbeterd om te garanderen dat deze voldoet aan de voortdurend toenemende vereisten voor betrouwbaarheid en nauwkeurigheid die door de automobieltoepassingen gevergd worden. Dit onderzoek stelt een AMR-sensorsysteem voor, met een fouttolerante benadering voor compensatie van catastrofale fouten en self-X-eigenschappen om de sensorprestaties tijdens de gehele levensduur constant te houden. Daarnaast is een interface conform de IEEE 1687-standaard overwogen, die de sensor in staat stelt te communiceren met andere componenten van het systeem waarin deze is geïntegreerd.

Self-X-eigenschappen omvatten het vermogen van een systeem om bepaalde functies zelfstandig uit te voeren, zonder assistentie van buiten het systeem. In ons systeem wordt een self-monitoringfunctie van de maximale hoekfout voorgesteld om te bepalen of verouderingscompensatie nodig is. In dat geval maakt onze zelfkalibratiemethode het bijwerken van de compensatiefactoren voor offsetvoltage en amplitude-onbalans mogelijk. Dankzij onze fouttolerante benadering kan de sensor blijven functioneren, zelfs als er een catastrofale storing optreedt in de magnetoweerstanden of de voedings- of aardeaansluitingen. Dit alles vindt online plaats op grond van digitale dataverwerking gedurende de sensorlevensduur. Het systeem werd geverifieerd aan de hand van gegevens die uit een analysemodel van de sensor verkregen zijn, maar ook aan de hand van gegevens die in commerciële AMR-sensoren gemeten werden. De betrouwbaarheidsbeoordeling was voornamelijk gericht op de betrouwbaarheidskenmerken, bedrijfszekerheid, veiligheid, onderhoudbaarheid en beschikbaarheid om na te gaan welke verbetering van de betrouwbaarheid kan worden gerealiseerd met het voorgestelde systeem.

Samenvattend, wordt er een AMR-sensorsysteem voor hoekmetingen in automobieltoepassingen voorgesteld, waarmee een juiste werking van de sensor gegarandeerd kan worden, zelfs als er onverwachte storingen of ongewenste verouderingseffecten optreden. Dit is meer dan ooit van belang, gezien de huidige trend waarbij men voor het juiste onderhoud aan auto's voornamelijk van elektronische systemen gebruikmaakt. 


\section{Acknowledgment}

This dissertation represents the end of a journey that started in 2013 when I saw a call for a PhD position at the University of Twente in the Netherlands. I would like to express my sincere gratitude to those who supported me in one way or the other during this amazing journey.

First, I would like to thank my supervisor Dr. Hans Kerkhoff for his valuable guidance during my research and writing of this dissertation. My sincere thanks also go to Prof. Gerard Smit for giving me the opportunity to join the CAES group and for helping me with the revision of my dissertation. In addition, I would like to thank Frans de Jong and Klaus Reimann for their guidance and support during the time I made measurements in NXP Semiconductors.

I would also like to thank my former colleagues for the time shared in the CAES group and in especial to Alireza Rohani, Jinbo Wan, Ahmed Ibrahim, Yong Zhao, and Hassan Ebrahimi. Very special thanks to Marlous Weghorst, Thelma Nordholt, and Nicole Baveld for their valuable support in all administrative tasks in the CAES group.

Last but not the least; I would like to thank my family for the unconditional support. To my parents, I could never achieve this goal without you.

Andreina Zambrano, September 2018. 
This page intentionally left blank. 


\section{Contents}

$\begin{array}{ll}\text { Acronyms } & \text { iii }\end{array}$

1 Introduction $\quad 1$

1.1 Problem statement and research question . . . . . . . . . . . . . . 4

1.2 Thesis organization . . . . . . . . . . . . . . . 7

References ...................... 8

2 Background and State-of-the-Art $\quad 11$

2.1 Introduction . . . . . . . . . . . . . . . . . . . . 11

2.2 AMR sensors . . . . . . . . . . . . . . . . . . . 12

2.2.1 Introduction . . . . . . . . . . . . . . . . . . . 12

2.2.2 AMR sensors for angle measurements . . . . . . . . . . . . . 14

2.3 Dependability . . . . . . . . . . . . . . . . . . . 18

2.3.1 Self-X properties .................... 20

2.3.2 Fault-tolerance . . . . . . . . . . . . . . . . 21

2.4 Dependability requirements in automotive applications . . . . . . . 24

2.5 Conclusions . . . . . . . . . . . . . . . . . . 25

References . . . . . . . . . . . . . . . . 27

3 Characterization of AMR sensors 31

3.1 Introduction . . . . . . . . . . . . . . . . . . . . 31

3.2 Parameters to characterize the AMR sensors . . . . . . . . . . . . 33

3.2.1 Anisotropic Magnetoresistance . . . . . . . . . . . . . . 33

3.2 .2 Offset Voltage . . . . . . . . . . . . . . . . . . 37

3.2.3 Amplitude of the sinusoidal signals at the outputs of AMR sensors . . . . . . . . . . . . . . . . 38

3.2.4 Angle error . . . . . . . . . . . . . . . . . . . . 40 40

3.3 Effects of aging on AMR sensors . . . . . . . . . . . . . . . . 42

3.3.1 The first aging test . . . . . . . . . . . . . . . . 44

3.3.2 The second aging test . . . . . . . . . . . . . . . . 48

3.3.3 Performance degradation . . . . . . . . . . . . . . . 66

3.4 Conclusions . . . . . . . . . . . . . . . . . . . . 67

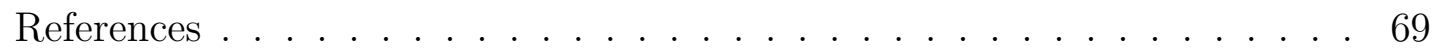

4 Self-X properties of AMR sensors $\quad 71$

4.1 Introduction . . . . . . . . . . . . . . . . . . 71

4.2 Self-Monitoring . . . . . . . . . . . . . . . . . . 72 
4.2 .1 Introduction . . . . . . . . . . . . . . . . . 72

4.2.2 MATLAB implementation . . . . . . . . . . . . . . . 78

4.3 Self-Calibration . . . . . . . . . . . . . . . . . . . 82

4.3 .1 Introduction . . . . . . . . . . . . . . 82

4.3 .2 Proposed method . . . . . . . . . . . . . . . 82

4.3.3 MATLAB implementation . . . . . . . . . . . . . . 90

4.3.4 FPGA implementation .................. 94

4.4 Conclusions . . . . . . . . . . . . . . . . . . . . . . . . . . . . . . . . . . . . . . . . . . . . . .

References . . . . . . . . . . . . . . . . . 105

5 Fault-tolerance in AMR sensors $\quad 107$

5.1 Introduction . . . . . . . . . . . . . . . . . . . 107

5.2 Catastrophic faults in AMR sensors . . . . . . . . . . . . . . . 109

5.2.1 The effect of catastrophic faults in AMR sensors . . . . . . . . 111

5.3 Fault-tolerant system for AMR sensors . . . . . . . . . . . . . . . 115

5.3 .1 Introduction . . . . . . . . . . . . . . . . . . . . . . . 115

5.3.2 Proposed fault-tolerant system . . . . . . . . . . . . . 116

5.4 Conclusions . . . . . . . . . . . . . . . . . . . . 130

References . . . . . . . . . . . . . . . . . . 132

6 A dependable AMR sensor system $\quad \mathbf{1 3 5}$

6.1 Introduction . . . . . . . . . . . . . . . . . . . 135

6.2 The dependability approach . . . . . . . . . . . . . . . . . 136

6.3 Architecture . . . . . . . . . . . . . . . . . . 137

6.3.1 The compensation module . . . . . . . . . . . . . 139

6.3.2 The manager . . . . . . . . . . . . . . . . . . . . . . . 139

6.3.3 The performance monitoring module . . . . . . . . . . . 140

6.3.4 The fault-tolerance module . . . . . . . . . . . . . . . . 141

6.3.5 The IEEE 1687 module . . . . . . . . . . . . . . . . . . . . . 143

6.4 Dependability assessment . . . . . . . . . . . . . . . . . . . 147

6.4 .1 Introduction . . . . . . . . . . . . . . . . . 147

6.4.2 Dependability assessment of the proposed AMR sensor system 149

6.5 Conclusions . . . . . . . . . . . . . . . . . . . . . 158

References . . . . . . . . . . . . . . . . . . . 159

7 Conclusions and Recommendations $\quad 161$

7.1 Introduction . . . . . . . . . . . . . . . . . . . . . . 161

7.2 Contributions . . . . . . . . . . . . . . . . . 161

7.2.1 Aging effects on AMR sensors for angle measurements . . . . 161

7.2.2 Aging compensation in AMR sensors . . . . . . . . . . . . . 162

7.2.3 Fault-tolerant system for AMR sensors . . . . . . . . . . . . 162

7.2.4 Architecture of the proposed AMR sensor system . . . . . . . 163

7.3 Conclusions . . . . . . . . . . . . . . . . . . . . . . . . . 163

7.4 Recommendations for future work . . . . . . . . . . . . . 165

List of Publications . . . . . . . . . . . . . . . . 166

$\begin{array}{ll}\text { Appendices } & 167\end{array}$ 


\section{Acronyms}

ADC Analog-to-Digital Converter.

ADT Accelerated Degradation Test.

AMR Anisotropic MagnetoResistance.

ASIL Automotive Safety Integrity Levels.

BISCA Built-In Self-Counter Actions.

BISD Built-In Self-Diagnostics.

CAT Configurable Analog Transistors.

CDM Cluster Dependability Manager.

CORDIC Coordinate Rotation Digital Computer.

CSADT Constant Stress Accelerated Degradation Test.

CSIB Cluster Segment Insertion Bit.

DM Dependability Manager.

DMM Digital Multimeter.

DMR Dual Modular Redundancy.

DRAM Dynamic Random Access Memory.

DSIB Dependability Manager Segment Insertion Bit.

ECU Electronic Control Unit.

FFT Fast Fourier Transform.

FO Failt-operational.

FPGA Field-Programmable Gate Array.

JTAG Joint Test Action Group.

MTTF Mean Time to Failure. 
NPI Non-Preemtive Interrupt.

PI Preemtive Interrupt.

RWA Road-Wheel Angle.

SBI Segment Insertion Bit.

SBW Steer-by-Wire.

SMU Source Measurement Unit.

SDM System Dependability Manager.

SSADT Step Stress Accelerated Degradation Test.

STD Standard Deviation

TC Temperature coefficient.

TDI Test Data Input.

TDO Test Data Output.

TDR Test Data Register.

TMR Triple Modular Redundancy.

VHDL VHSIC (Very High Speed Integrated Circuit) Hardware Description Language.

VI Virtual Instrument. 


\section{Chapter 1}

\section{Introduction}

The usage of technology is continually increasing in everyday life of human beings. Electronic systems currently support applications ranging from industrial, medical, household to automotive. Modern cars have been transformed from primarily mechanical entities to complex embedded systems running on four wheels [Cha15], [Buj04]. Figure 1.1 shows the percentual growth of the cost of electronic components compared with the total cost of the car. The proportion of the cost of electronic components was $35 \%$ in 2010, but for 2030 it is expected it will increase up to $50 \%$ at least. Eighty percent of innovation in the automotive field is driven by electronic inventions, which is projected to continue, especially with the current trend of autonomous electric cars [Nel10].

The growing usage of electronic components in automotive is driven by several concurrent forces including energy efficiency, comfort, performance improvement of the car and safety. The World Health Organization (WHO) states that 1.2 million people are killed on world roads every year; the number is expected to rise to 1.9 million by 2020 [WHO15]. The goal is that in the future, car manufacturers will put even more effort in techniques to avoid accidents, protect and entertain its occupants, as well as be more friendly with the environment [Cho10].

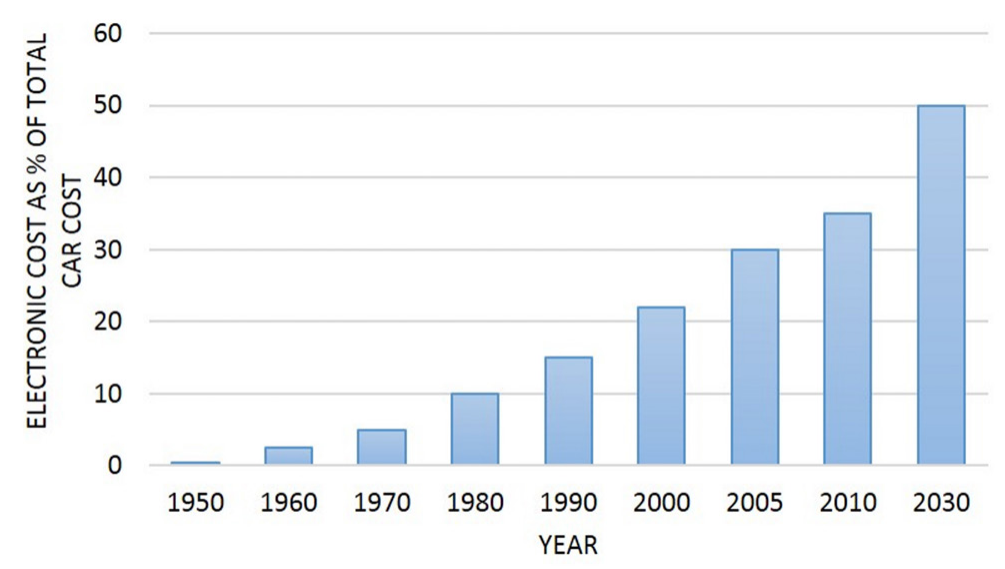

Figure 1.1: Growth of electronic content in automotive [Nel10]. 
In general, allowing electronic systems to control the engine and other automotive systems has helped to make today's cars and trucks safer, more fuel efficient and even more reliable. Nowadays, high-end cars include several electronic systems, as shown in Figure 1.2. They can be directly related to the movement of the car. Traditionally used mechanical and hydraulic systems, have been replaced by electronic systems called X-by-wire systems, such as brake-by-wire and steer-by-wire system. These are considered safety-critical because faults can represent a threat to humans or the environment [Kop99]. Consequently, these electronic systems should be trusted to perform the correct service or take the required actions to stay in a safe state in case a failure occurs. The standard ISO 26262 is focussed on the design of electronic systems for automotive applications, which can prevent critical failures or take the required actions to stay in a safe state when a failure occurs [Sin11].

As the number of electronic components capable of taking control of the throttle, brakes and other safety-critical systems increases, it is more important than ever to be sure that these elements will work together safely and predictably. Therefore ensuring dependability is of increasing importance, as it represents the trust that a user justifiably poses on the service offered by the system [Buj04], [Cha04]. X-by-wire systems consist of sensors, electronic control units (ECU) and front-end electronics (analog amplifier, analog-to-digital converter,etc) connected through bus networks, as presented in Figure 1.3. It shows a steering-by-wire system and a brake-by-wire system with an ECU per wheel [Kaw17], [Pel17]. Hence, to have a dependable system it is required high-performance electronic components capable of operating for a long time in a highly dependable manner.

The dependability of a system could be enforced by techniques able to prevent, tolerate and remove failures [Buj04], [Ise02]. However, the development of a dependable X-by-wire system is more challenging compared to other safety-critical applications due to the specific characteristics of the automotive field, as detailed below [Plo08], [Man01], [Kop99]:

- Harsh operational conditions with temperatures from -40 to $+175{ }^{\circ} \mathrm{C}$, temperature shocks, vibration, moisture, etc.

- The system design is driven by the often conflicting demands of superior functionality, high dependability, and minimal cost.

- Automotive electronics, unlike consumer electronics, need to remain in operation for 15 years.

- As the accuracy and dependability requirements are continually increasing, semiconductor technologies used in cars are becoming more sophisticated and more exposed to reliability and aging issues. Consequently, the need for self-configuration and plug-and-play components is ever increasing. 


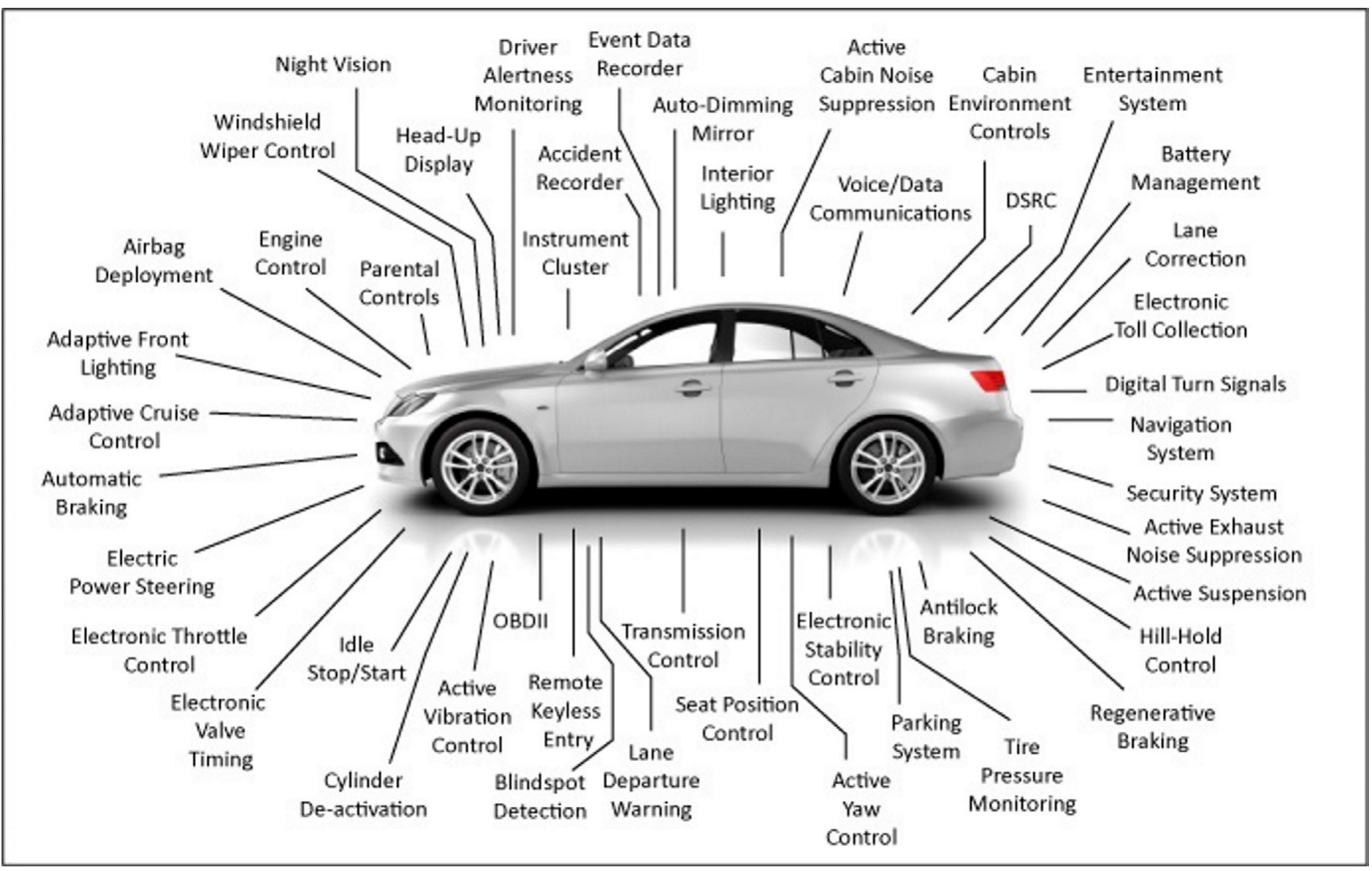

Figure 1.2: Electronic systems in a car [Inn15]. 


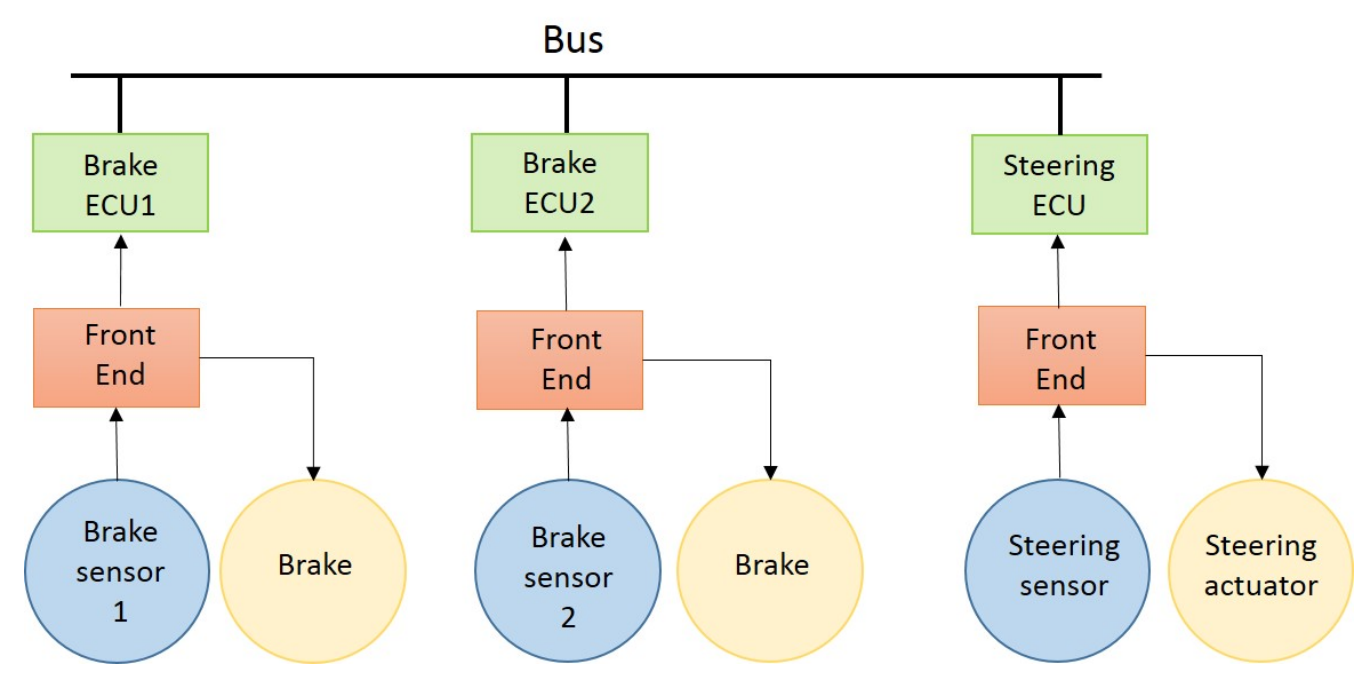

Figure 1.3: Possible architecture of X-by-wire systems.

\subsection{Problem statement and research question}

The increasing usage of electronic systems in automotive applications also implies an increasing number of automotive sensors. An average car on the road today has around 30 sensors for gathering information regarding speed, temperature, pressure, torque, oxygen levels, and more as shown in Figure 1.4. This information is sent to ECUs for processing and determining the following actions that should be performed [Kaw17]. Safety-critical systems demand dependable, high-performance and low cost sensors. Magnetic sensors represent an excellent option to be applied in almost all cases in which wear-affected potentiometric techniques are traditionally used; examples are pedal position, engine control and transmission control [Tre01], [Buj04]. They offer several key advantages including mechanical robustness due to their non-contact measurement principle, a very wide operating temperature range, and low manufacturing costs [Bar06].

Magnetic sensors based on the Anisotropic MagnetoResistance effect (AMR sensors) are often used for angle measurements. The sensor is configured with two Wheatstone bridges placed under an angle of $45^{\circ}$ with respect to each other. The bridge outputs should be two perfect sinusoidal signals that depend on the angle to be measured, which is generated by a permanent magnet on top of the sensor. However, the actual outputs include undesired parameters such as offset voltage, amplitude imbalance and additional harmonics that affect the accuracy of the calculated angle and hence the performance of the sensor [Die00]. 


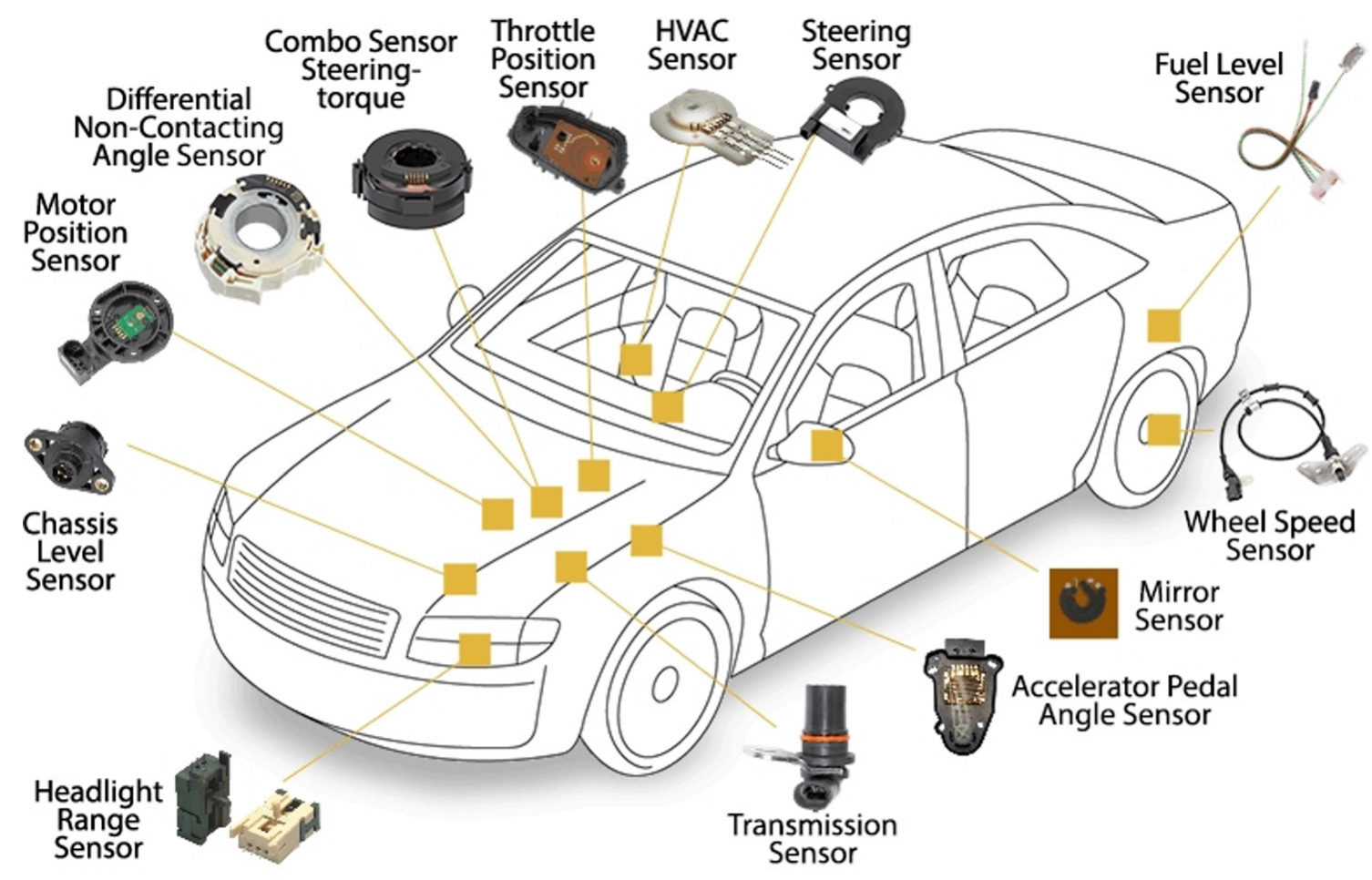

Figure 1.4: Some automotive sensors in a car [Bou15]. 
Until now, the offset voltage is the parameter mainly compensated during final test because it is the largest source of angle error. Most of the proposed compensation methods are aimed to calculate the required compensation factors in factory conditions, as detailed in chapter 2. The compensation factors are estimated at the start of the sensor life but are not updated during its lifetime. Although the offset voltage drifts due to wearing and aging effects, it remains within the allowed tolerance band nowadays. Isler stated in [Is110] that the drift of the offset voltage over a thousand hours at high temperature should be in the range of some tens of $\mu \mathrm{V} / \mathrm{V}$, which can be considered as less than $100 \mu \mathrm{V} / \mathrm{V}$ to guarantee an accuracy better than $1^{\circ}$ over the sensor's lifetime. However, the dependability and accuracy requirements in automotive applications are continually increasing, especially with the current trend towards autonomous vehicles [Ham03]. This means that the tolerance band for drifting will become increasingly narrow (less than $50 \mu \mathrm{V} / \mathrm{V}$ ). Nevertheless, is not yet clear whether it will be necessary to compensate the aging effects to guarantee the performance of the sensor. This also applies to the undesired parameters of amplitude imbalance and additional harmonics, as will be discussed later. To the best of our knowledge, little data has been published regarding the performance degradation of AMR sensors for angle measurements and the aging effects.

Besides performance degradation, an AMR sensor can also be affected by catastrophic faults resulting from a short or broken condition at any of the magnetoresistances of the sensor, loss of the magnet that generates the magnetic field which angle is to be measured or broken sensor connections to the power supply or ground [Die00]. This brings as a consequence that the sensor stops working because the angle cannot be calculated any longer.

Dependability, as well as the accuracy requirements demanded by automotive applications are constantly increasing [Cha15]. Therefore, to guarantee that AMR sensors will satisfy these requirements, it is necessary to embrace strategies that allow the sensor to handle performance degradation as well as catastrophic faults. This thesis will focus on the development of a dependable AMR sensor system for angle measurements. The problem statements can be specified in more detail as follows:

1. Wearing and aging effects will affect the accuracy of the sensors in the future. Therefore it is important to have a better understanding of the aging effects to define a proper methodology to perform aging compensation. Hence, an aging test focused on the performance degradation of the sensor should be developed.

2. In order to define the best method to perform aging compensation, it is important to consider strategies that allow the sensor to keep its performance over time as well as the operational conditions of the sensor. During its lifetime a particular setup is defined and limited computational processing power should be available.

3. The sensor operation should be guaranteed despite the occurrence of a catas- 
trophic fault occurs. Therefore, the proposed system should include a faulttolerant method.

4. The electronic systems included in a car should cooperate and interact smoothly to provide the expected transportation service. Therefore, it is important to provide the sensor with the capability to communicate with other components of the X-by-wire system in which it is integrated.

\subsection{Thesis organization}

The remainder of this thesis has been organised as follows. Chapter 2 presents the background as well as the state-of-the-art regarding AMR sensors, dependability, its attributes, dependability means used in this research and dependability requirements in automotive applications. Chapter 3 focusses on the study of aging effects on the performance of the sensor. Therefore a set of commercial AMR sensors made by NXP has been characterised at the start of their life but also during the execution of aging tests. Chapter 4 proposes the use of self-X properties to keep the sensor performance over time. Methods for self-monitoring and self-calibration are suggested to update online the compensation factors when required during the sensor lifetime. In Chapter 5 a fault-tolerant method is introduced to avoid the sensor from stopping its operation in case a catastrophic fault occurs. Chapter 6 proposes an architecture for the dependable AMR sensor system, which includes the methods proposed in the previous sections, but also a dependability manager to control all the activities of the system and an interface to the IEEE 1687 standard that handles the connection with external components. Besides, it is presented the dependability improvement can be achieved with the proposed system regarding the dependability attributes. Chapter 7 concludes the thesis with a summary of the presented work and gives some suggestions for future work. 


\section{References}

[Bar06] A. Bartos, A. Meisenberg, and R. Noetzel. "Novel Redundant Magnetoresistive Angle Sensors". Sensoren und Messysteme, pp. 99-102, 2006.

[Bou15] Bourns. "http://www.bourns.com/products/automotive/automotivesensors", 2015. Accessed 2017-03-20.

[Buj04] G. Buja, S. Castellan, R. Menis, and A. Zuccollo. "Dependability of safety-critical systems". In IEEE International Conference on Industrial Technology, volume 3, pp. 1561-1566, Dec 2004.

[Cha04] F. Charfi and F. Sellami. "Overview on dependable embedded systems in modern automotive". In IEEE International Conference on Industrial Technology, volume 2, pp. 781-786, Dec 2004.

[Cha15] S. Chakraborty and S. Ramesh. "Guest Editorial Special Section on Automotive Embedded Systems and Software". In IEEE Transactions on Computer-Aided Design of Integrated Circuits and Systems, volume 34(11), pp. 1701-1703, Nov 2015.

[Cho10] A. Chong. "The growth of automotive electronic in APAC, the next frontier". In Driving Asia - As Automotive Electronics Transforms a Region. Infineon Technologies Asia Pacific Pte Ltdr, 2010.

[Die00] K. Dietmayer and M. Weser. "Contactless Angle Measurement using KMZ41 and UZZ9000". Application Note. AN00023, Philips Semiconductors, Germany, 2000.

[Ham03] R. Hammett and P. Babcock. "Achieving 10-9 Dependability with Driveby-Wire Systems". SAE Technical Paper 2003-01-1290, pp. 157-170, March 2003.

[Inn15] InnovationIntellienge. "http://innovationintelligence.com/thought-leaderthursday-model-based-embedded-design/", 2015. Accessed 2017-03-20.

[Ise02] R. Isermann, R. Schwarz, and S. Stolzl. "Fault-tolerant drive-by-wire systems". IEEE Control Systems, volume 22(5), pp. 64-81, Oct 2002.

[Isl10] M. Isler, B. Christoffer, G. Schoer, B. Philippsen, et al. "Optimisation of surface passivation for highly reliable angular AMR sensors". Physica status solidi (c), volume 7(2), pp. 436-439, 2010.

[Kaw17] N. Kawahara and K. Hashmi. "Automotive Applications". In Reference Module in Materials Science and Materials Engineering. Elsevier, 2017.

[Kop99] H. Kopetz. "Automotive electronics". Proceedings of the 11th Euromicro Conference on Real-Time Systems, pp. 132-140, 1999. 
[Man01] A. Manzone, A. Pincetti, and D. D. Costantini. "Fault tolerant automotive systems: an overview". Proceedings Seventh International On-Line Testing Workshop, pp. 117-121, 2001.

[Nel10] S. Nelson. "Automotive Market and Industry Update". Technical report, Freescale Semiconductor, 2010.

[Pel17] P. Pelliccione, E. Knauss, R. Heldal, S. M. Ågren, et al. "Automotive Architecture Framework: The experience of Volvo Cars". Journal of Systems Architecture, volume 77, pp. 83-100, 2017.

[Plo08] R. Ploss, A. Mueller, and P. Leteinturier. "Solving automotive challenges with Electronics". In International Symposium on VLSI Technology, Systems and Applications (VLSI-TSA), pp. 1-2, April 2008.

[Sin11] P. Sinha. "Architectural design and reliability analysis of a fail-operational brake-by-wire system from ISO 26262 perspectives". Reliability Engineering and System Safety, volume 96, pp. 1349-1359, 2011.

[Tre01] C. Treutler. "Magnetic sensors for automotive applications". Sensors and Actuators A: Physical, volume 91(1-2), pp. 2-6, 2001.

[WHO15] WHO. "Global status report on road safety". Technical report, World Health Organization, 2015. 
This page intentionally left blank. 


\title{
Chapter 2
}

\section{Background and State-of-the-Art}

\begin{abstract}
In this chapter, AMR sensors are explained in detail, including previous research focussed on the improvement of the sensor accuracy and the study of the sensor performance over time. Next, the background and state-of-the-art are presented regarding dependability, and its attributes. Furthermore, the means that can be used to improve the dependability of a system are explained as well as the dependability requirements of automotive applications.
\end{abstract}

\section{$2.1 \quad$ Introduction}

The increasing introduction of electronic systems in the automotive field will further improve driver safety, as well as comfort, engine efficiency and the performance of cars. However, these systems demand reliable, high performance, and low-cost electronic components that are capable of operating for a long time in a highly dependable manner [Man01]. Therefore, the sensors required in a car should be reliable to provide the correct service despite the sometimes harsh operating conditions. AMR sensors represent an excellent option to be applied in cases in which wear-affected potentiometers are traditionally used [Tre01]. This chapter is focussed on presenting the background as well as previous research regarding AMR sensors and dependability means which can be applied to improve the dependability of this type of sensors.

The rest of the chapter is organized as follows. Section 2.2 introduces the AMR sensor, its working principle and components. Furthermore, it explains how an AMR sensor is configured for angle measurements, and the characteristics of the sensor outputs and the sensor performance are presented. Section 2.3 briefly discusses the concept of dependability, its attributes and dependability means used in our research. Section 2.4 focusses on the dependability requirements of current and future automotive applications and section 2.5 provides the conclusions. 


\section{$2.2 \quad$ AMR sensors}

\subsubsection{Introduction}

An anisotropic magnetoresistance (AMR) sensor is a type of magnetic sensor. Its working principle is based on the magnetoresistive effect, a property of some magnetic materials, in which the resistance value changes due to a varying magnetic field [Car98]. The sensor has been introduced in a wide range of applications in automotive, consumer electronics and biotechnology. This is a result of the simplicity of its design, low cost, robustness and temperature stability [Bat07].

The sensor is made of a simple layered stack consisting of a thin magnetic material layer on a semiconductor substrate, a metal layer for bondpads and connections, and a passivation layer for protection. The most common magnetic material used is permalloy, an alloy of $80 \%$ nickel and $20 \%$ iron (NiFe). It is characterised by its mechanical robustness and magnetic properties of near-zero magnetostriction, and significant anisotropic magnetoresistance value [Hau00]. In automotive applications, the passivation layer $(\mathrm{SiN})$ on top of the NiFe layer should be moisture resistant to fulfil the demanding and continuously growing automotive reliability and accuracy requirements [Len06], [Isl10].

The ohmic resistance value of the permalloy material depends on the angle $(\theta)$, between its internal magnetisation vector and the direction of the current flow. The magnetisation vector refers to the direction of the magnetic dipoles of the material. During the sensor fabrication, the permalloy is deposited in a strong magnetic field that sets the preferred orientation, or easy axis of the magnetization vector parallel to the length of the permalloy resistor. However, if an external magnetic field $\mathrm{H}$ is applied parallel to the permalloy, the internal magnetisation vector rotates towards the direction of the magnetic field, as shown in Figure 2.1 [Car98], [Len06].

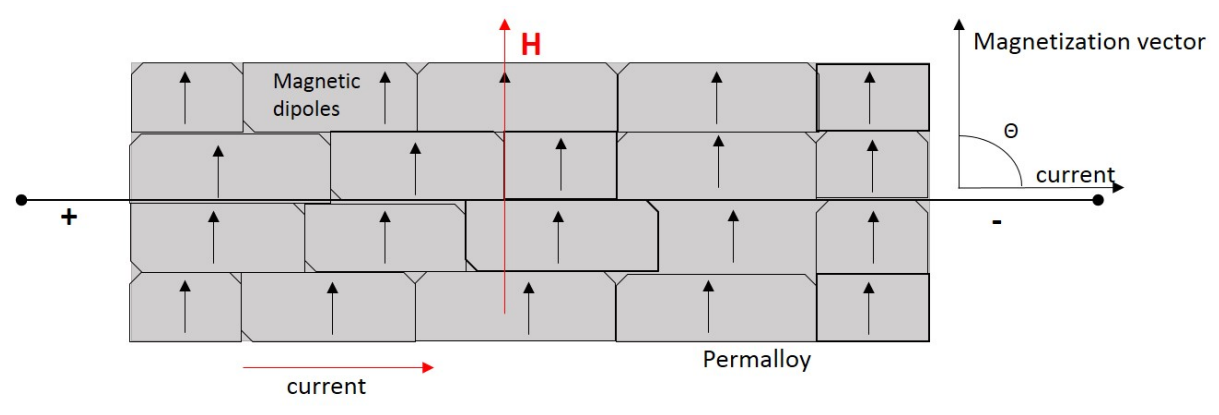

Figure 2.1: The magnetoresistive effect in permalloy [Die00]. $\mathrm{H}$ represents an external magnetic field, e.g. via a magnet.

The resistance $\mathrm{R}$ of the material changes depending on the rotation angle, as presented in Equation (2.1). Ro and $\triangle R o$ represent material constants, being $\triangle R o$ in the order of 2 to $3 \%$ of Ro. The resistance value is at its maximum at an angle $\theta$ 


\subsection{AMR sensors}

of $0^{\circ}$ and at its minimum at $90^{\circ}[\mathrm{Die} 00]$.

$$
R=R o+\triangle R o \cdot \cos (\theta)^{2}
$$

Usually, four magnetoresistive elements are electrically connected as a Wheatstone bridge. In theory, the bridge resistances have the same value forming diagonal pairs of identical elements that react oppositely to one another to an external magnetic field, as depicted in Figure 2.2. H represents the external magnetic field, I the electric current that flows through the magnetoresistive elements. For example, if R21 and $\mathrm{R} 23$ are at their minimum values, R22 and R24 are at their maximum values. This type of configuration allows optimising the output voltage of the sensor.

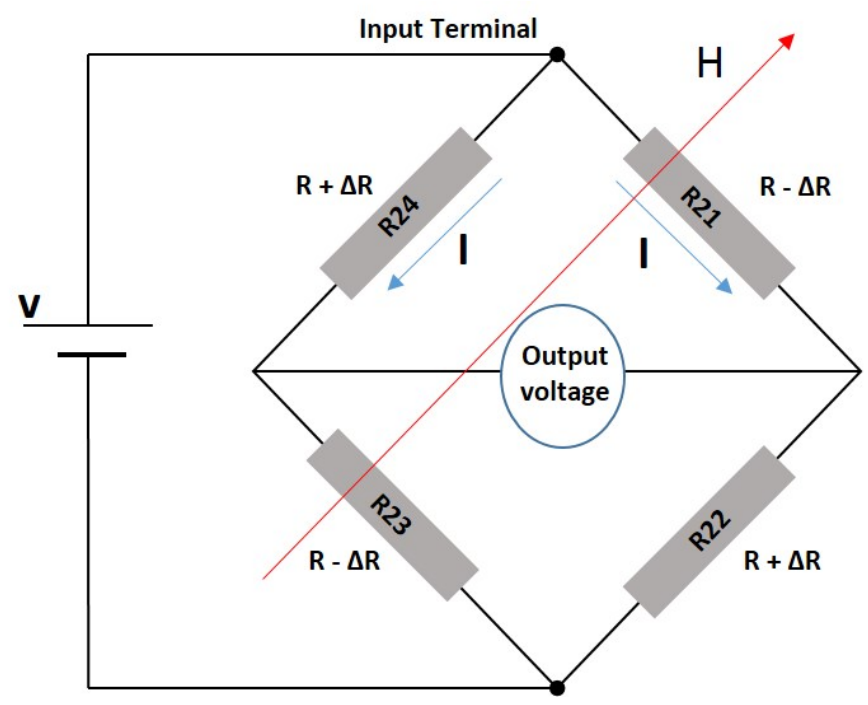

Figure 2.2: Schematic of a Wheatstone bridge in an AMR sensor.

AMR sensors can be divided into two groups. One is aimed to measure the strength of low magnetic fields, while the other provides the angle of high magnetic fields. For low-field applications, the sensor is configured with one Wheatstone bridge operating in a region in which there is a linear relationship between the resistance variation and the magnetic field. The linearization is achieved by depositing aluminium stripes (Barber poles) on top of the permalloy strip at an angle of $45^{\circ}$ to the strip axis [Bar08], [Phi00].

For angle-measurement applications, the applied magnetic field should be sufficiently strong to saturate the magnetic material of the sensor (roughly $\mathrm{H}>10$ $\mathrm{kA} / \mathrm{m}$ ). This to guarantee that the magnetic dipoles of the permalloy are aligned and following the external magnetic field. As a result, the resistances in the sensor change as a function of the angle between the external field and the current flow. 


\subsubsection{AMR sensors for angle measurements}

The magnetoresistive effect is an angular effect by nature. Therefore, AMR sensors are recommended for angle-measurement applications. An AMR sensor for angle measurements consists of eight magnetoresistances configured into two Wheatstone bridges positioned at an offset angle of $45^{\circ}$ with respect to each other, as shown in Figure 2.3. In saturation state of the sensor, its magnetisation vector follows the magnetic vector of an external magnetic field usually generated by a permanent magnet on top of the sensor, which defines the angle to be measured.

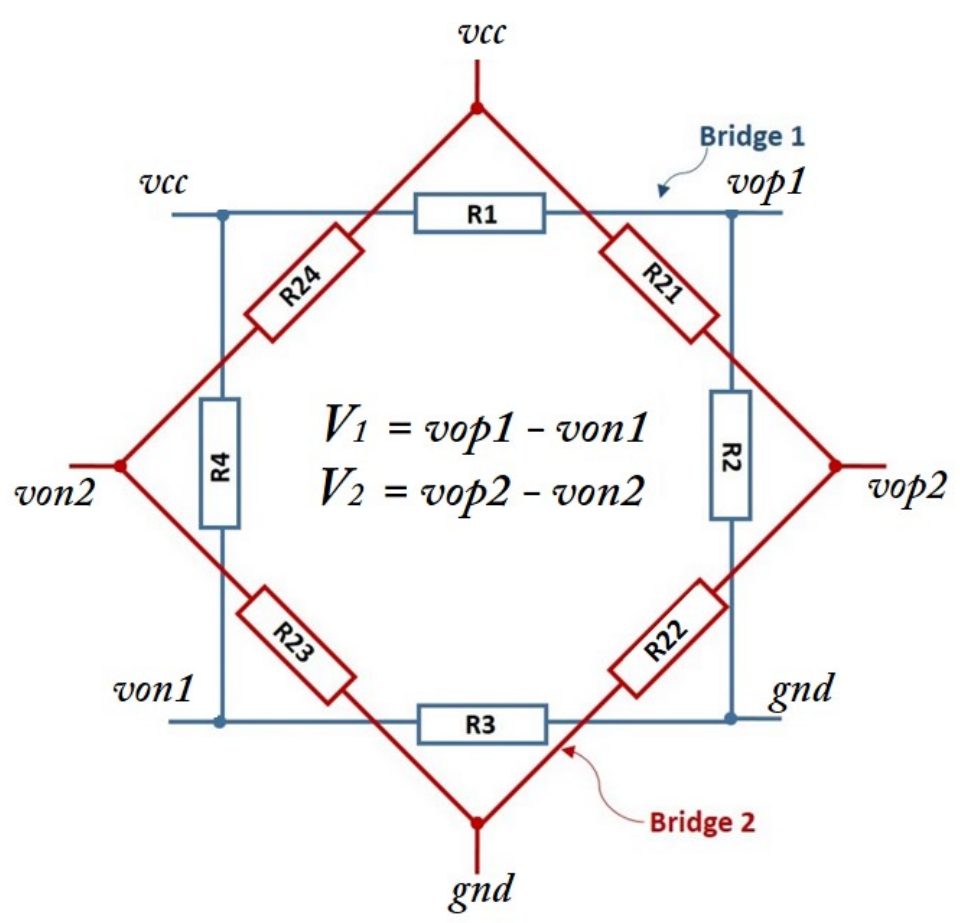

Figure 2.3: Schematic of the Wheatstone bridges in an AMR sensor. Each $\mathbf{R}$ represents a magnetoresistance.

Due to the sensor configuration, bridge 1 and 2 show output signals proportional to $\cos (2 \theta)$ and $\sin (2 \theta)$ respectively. As both signals depend on the magnetic angle $\theta$, they can be used to estimate an angular range between 0 and $180^{\circ}$ based on Equation (2.2) [Fel04]. In the sensor operational conditions, the angle is calculated with the CORDIC (Coordinate Rotation Digital Computer) algorithm. It is a simple and efficient algorithm that uses simple shift-add operations to calculate trigonometric, hyperbolic and logarithmic functions, etc [Zhu16].

$$
2 \theta=\arctan \left(\frac{V 2}{V 1}\right)=\arctan \left(\frac{v o p 2-v o n 2}{\operatorname{vop} 1-\operatorname{von} 1}\right)=\arctan \left(\frac{A * \sin (2 \theta)}{A * \cos (2 \theta)}\right)
$$




\subsection{AMR sensors}

A magnetoresistance is temperature dependent as detailed in [Vop13]. Therefore, the output voltage of a Wheatstone bridge is temperature dependent as well. However, in AMR sensors for angle measurements the angle calculation depends on the relationship between the output voltages of the two bridges and not on the amplitude of each signal. Since this relationship does not change with temperature, a temperature measurement or compensation of temperature effects is not required.

The two Wheatstone bridges are made on the same substrate in thin-film technology as shown in the layout of a sensor in Figure 2.4 [Die00]. Therefore, both bridges show a very good matching regarding electrical and mechanical properties. In theory, the bridge resistances have the same components. However, due to imperfections during the sensor manufacturing or assembly, a resistance mismatch exists in practice [Is110]. This has as a consequence that the actual sensor outputs include a number of undesired parameters. An extra voltage defined as offset voltage is always present at the bridge outputs, even if the four resistances sense the same angle, in which case the output voltage should be zero because the bridge resistances have the same value. The two sinusoidal output signals do not show the same amplitude (A1, A2) and additional harmonics may also be present. As a consequence, Equation (2.2) should be rewritten as presented in Equation (2.3) .

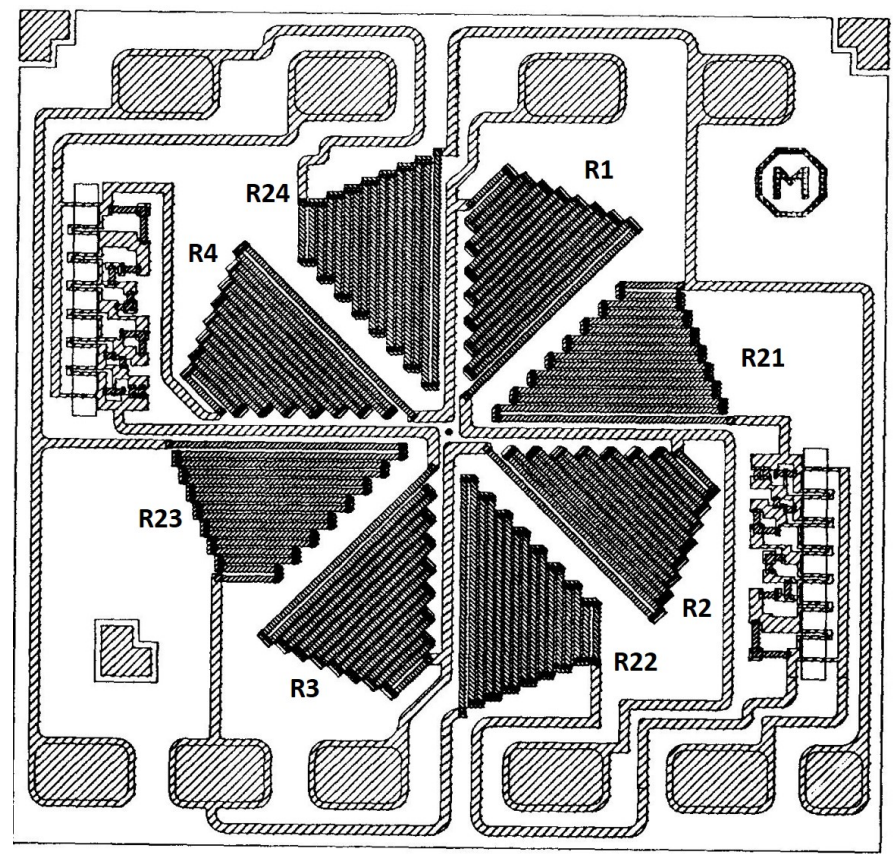

Figure 2.4: Layout of an AMR sensor for angle measurements, which includes eight magnetoresistances and the required connections to configure the two Wheatstone bridges [Die00].

$$
2 \theta=\arctan \left(\frac{A 2 * \sin (2 \theta)+\text { off } \operatorname{set} 2+N 2}{A 1 * \cos (2 \theta)+\text { off } \operatorname{set} 1+N 1}\right)
$$


where A1 and A2 denote the amplitude of the sine and cosine signal, offset1 and offset2 represent the DC offset voltages and N1 and N2 represent additional harmonics at the output voltage of bridge 1 and bridge 2 respectively. These extra components are sources of error in the angle calculation. They do not have a fixed value, but depend on the magnetic angle, showing sinusoidal characteristics as is shown in Table 2.1 [Lar14], [Lin11].

Table 2.1: Expressions of the angle error for the error sources present in AMR sensors [Lar14]

\begin{tabular}{|l|c|}
\hline error source & angle error expression \\
\hline Offset voltage & $\Delta O \sin (\alpha)$ \\
Amplitude imbalance & $\Delta A \sin (2 \alpha)$ \\
Harmonic distortion & $-\sqrt{2} K_{o} \cos (\alpha+\pi / 4)-\sum_{n=2}^{\infty} K_{n} \sin [(n-1) \alpha]$ \\
\hline
\end{tabular}

- $\alpha$ is equal to $2 \theta$ that represents the angle detected by the sensor.

- $\Delta O$ is related to the ratio of the offset voltages in the sensor outputs.

- $\Delta A$ is related to the ratio of amplitude imbalance between the sensor outputs.

- $K_{o}, K_{n}$ represent harmonic coefficients

The error sources should be compensated in order to improve the sensor accuracy. Until now, the offset voltage is the parameter mainly compensated in the industry for being the largest contributor to the error in the angle calculation [Isl10]. Next, some methods will be presented which have been proposed to calculate the compensation factors for the offset voltage:

- Offset compensation by adding an external magnetic field which includes two modes. In the first mode, an additional DC external magnetic field is provided in a predetermined direction, which dominates over the magnetic field generated by the permanent magnet whose angle is to be measured. For the second mode, the additional external magnetic field is removed. In this case, the sensor outputs from the two modes are combined to determine the magnetic angle with compensated offset voltage. The external magnetic field in the first mode is preferably at least ten times larger than the magnetic field generated by the permanent magnet. This is a challenge during the sensor lifetime in which the permanent magnet can generate a magnetic field as large as $25 \mathrm{kA} / \mathrm{m}$ [Zie11].

- Offset compensation based on applying and removing a magnetic field which is composed of three steps for each Wheatstone bridge [Waf01]:

1. The Wheatstone bridge is acted upon by a direct magnetic field which is aligned in such a way that it is neither substantially perpendicular to any of the two resistance pairs of the Wheatstone bridge. 


\subsection{AMR sensors}

2. The direct magnetic field is removed.

3. An evaluation circuit determines an offset calibration signal from the bridge output without the direct magnetic field.

The best correspondence between the offset voltage and the bridge output is obtained at an angle of $45^{\circ}$ in bridge one and $90^{\circ}$ in bridge two. This method cannot be used during the sensor lifetime because the magnetic field is always present.

- Maximum and minimum approach. The offset voltage can be determined from the arithmetic mean of the extreme values of the sinusoidal signals at the sensor outputs, as indicated in Equation (2.4). In [Mut04] it has been proposed to execute a calibration cycle, in which the permanent magnet on top of the sensor is rotated during a measuring cycle. Then, by detecting the extreme values of the measurements it is possible to determine the offset voltage of each bridge, as indicated in Equation (2.4) for bridge 1 in which $V 1$ represents the output voltage of the bridge.

$$
\operatorname{offset} 1=\frac{\max (V 1)+\min (V 1)}{2}
$$

The main drawback of this method is the accuracy and time required to detect the extreme values of the output voltages of the bridges. Although these values can be carefully detected in controlled conditions in the factory, this is not the case during the sensor lifetime. Figure 2.5 shows the effect of the accuracy during the data acquisition over the detection of the real extreme values of the bridge outputs. An incorrect sampling of the data leads to the detection of false maximum and minimum values and hence an inaccurate offset voltage. For example, in Figure 2.5, using the real maximum and minimum values, the offset voltage is calculated to be $0.3 \mathrm{mV}$, while with the detected maximum and minimum values the offset voltage is $0.4 \mathrm{mV}$ instead.

The methods mentioned before could be very efficient to calculate compensation factors under factory conditions. However taking their requirements with regard to setup, external equipment (e.g extra magnetic field), processing or timing involved into consideration, these are not the best options to determine compensation factors online during the sensor lifetime. 


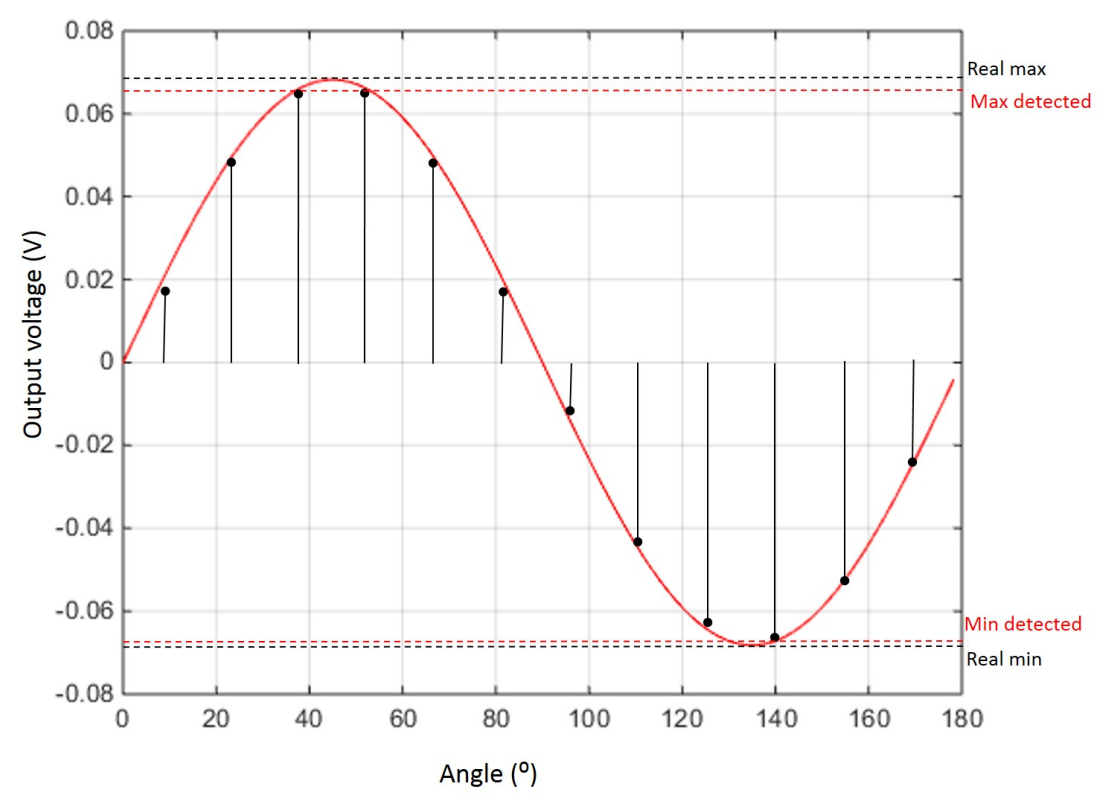

Figure 2.5: Effect on the accuracy in the data acquisition with respect to the detection of the real maximum values in case of sampling. Although the offset voltage is $0.3 \mathrm{mV}$, using the detected values the calculated offset voltage turns out to be $0.4 \mathrm{mV}$.

Until now, it is not considered necessary by manufacturers to update the compensation factors for offset voltage during the sensor lifetime, although it is known that offset voltage drift can occur due to wearing and aging effects. However, over the lifetime the sensor performance remains within the tolerance band currently permitted. To the best of our knowledge, there is hardly any published data regarding the performance degradation of AMR sensors due to aging effects. In [Ibe03], Iben studies the aging effects on AMR read sensors used in tape-storage drives; this research focussed on the bridge resistances and the amplitude of the sensor outputs. Isler reported in [Isl10] some data related to the drift of the offset voltage in one of the Wheatstone bridges of AMR sensors used for angle measurements.

In the future, it is expected that the tolerance band for drifting of the undesired parameters included at the sensor outputs will become increasingly narrow, especially with the current trend of autonomous cars [Zor17]. Therefore it will be important to have a better understanding of the aging effects in AMR sensors.

\subsection{Dependability}

The dependability of a system can be defined as its trustworthiness that in a given environment the system will operate as expected during its normal operation [Kha11]. It is an important requirement in safety-critical applications, where a failure of the system can endanger people, damage the environment or things [Buj04]. Depend- 
ability cannot be measured by one quantity but rather by several attributes such as [Ker10], [Abd06], [Kha14], [Sin11]:

- Reliability refers to the capability of a system to continue operating without a failure. High reliability is required in situations in which it is expected that a system operates without interruptions, for example a heart pacemaker; or if maintenance cannot be performed because the system cannot be accessed, as in deep-space applications. Reliability uses as metric the failure rate $(\lambda)$ that is defined as the number of failures per unit of time.

- Maintainability represents the capability of a system to undergo modifications and repairs. In an embedded IP or a SoC, a repair in the traditional sense is not feasible, but it is possible to detect a fault and perform countermeasures to guaranty the correct functionality of the system.

- Availability indicates the probability that a system will be available to deliver the correct service at any given time. It is often used for systems in which short interruptions can be tolerated, for example, networked systems. The amount of time the system is available is defined as uptime and the time is not available as downtime.

- Safety represents the probability that a system at time t either performs its function correctly or discontinues its operation in a fail-safe manner. This is an important attribute in safety-critical systems. For automotive applications, the standard ISO 26262 has been defined focused on the functional safety of electronic systems used in vehicles [Sin11]. This standard allows to design and assess systems that can prevent critical failures or take control if they occur. The safety requirement is based on four automotive safety integrity levels (ASILs) presented in Table 2.2. The lowest level is ASIL-A and the highest ASIL-D.

Table 2.2: Automotive Safety Integrity Levels (ASILs)

\begin{tabular}{|l|c|}
\hline ASIL level & Failure target value(failures $/ \mathbf{h})$ \\
\hline ASIL D & $<10^{-8}$ \\
ASIL C & $<10^{-7}$ \\
ASIL B & $<10^{-7}$ \\
ASIL A & $<10^{-6}$ \\
\hline
\end{tabular}

Dependable systems are developed using methods termed as dependable means, among which are fault-avoidance, fault-removal, self-X properties and fault-tolerance [Zor17]. These methods are often combined to obtain better results. In the remainder self-X properties and fault-tolerance are explained in more detail, as they have been used in this research. 


\subsubsection{Self-X properties}

The self-X concept is based on studies of self-organizing systems in the nervous system. It was initially introduced in organic computing. Self-organization means that the system structure arises without explicit pressure or involvement from outside. Therefore, the system has a sufficient degree of freedom to allow self-organizing behaviour for adapting to dynamically changing requirements of the execution environment. For example, a system can eliminate the effects of malfunctioning units without the requirement of any external assistance [Sch05].

The self-X concept represents the capabilities of a system to perform certain functions on its own without any external help. It includes properties such as selfmonitoring, self-calibration, self-healing, etc. From the perspective of sensors, self-X properties are aimed to ensure that the sensor operates under optimum conditions. These features allow to improve flexibility, accuracy and reduce vulnerability to deviations and drift caused by manufacturing imperfections, environmental changes and aging effects. It is desirable that self-X properties require a minimum of computational effort together with a minimum of additional hardware. The ideal case would be that additional components for self-X implementations are embedded in the sensor itself. Self-X features are very useful in systems in which it is complicated to predict the degradation level as is the case of magnetic sensors [Joh11a].

Most of the research efforts have been expended to implement self-X features at a system level. At the component level in [Joh11b], Johar proposed adding self-X properties to AMR sensors used to measure the strength of the magnetic field. The sensor is configured with one Wheatstone bridge; hence, the resistance dependency of temperature affects the measurements results. Because of this, several self-X properties have been implemented. Self-monitoring by observing the temperature, self-compensation by compensating the temperature influence at the sensor output and self-repair by performing a so-called flipping action in case of sensor saturation.

In [Die01] is proposed to add online-diagnosis capabilities to AMR sensors for angle measurements. Based on a sine/cosine signal evaluation using the CORDIC algorithm, it is possible to monitor and diagnose the status of the sensor and the signal-conditioning IC. The diagnostic capabilities include broken supply or broken ground connections, fail in one or both bridges, as well as the pre-amplifier or ADC in the input stage.

In [Ker10] it is proposed to add self-X properties to improve the dependability of digitally-assisted mixed-signal IPs. A BISD (Built-In Self-Diagnostics) IP monitors the required parameter(s) to detect aging in a selected IP. The BISCA (Built-In Self-Counter Actions) IP takes care of controlling the chosen parameters from the BISD to compensate for aging degradation. In case it is determined that compensation is outside the controlling range, an isolation step, bypass, or spare-resource procedure can be applied if the SoC infrastructure provides this option.

Rudolf suggested online calibration of ICs by employing Configurable Analog 


\subsection{Dependability}

Transistors (CAT) [Rud12]. The usage of CAT as a circuit-level calibration technique allows improving the reliability and performance of analog circuits over the operating temperature range in hostile environments.

\subsubsection{Fault-tolerance}

Fault-tolerance is aimed to deal with faults in order to avoid a system failure. This is especially important in safety-critical functions, which should not suddenly fail without pre-warning, meaning they should not have a single-point-of-failure [Pol95]. Fault-tolerance is usually implemented by space or time redundancy. This means there exists more than one way of performing a required function [Lay08]. Such redundant schemes can be designed for hardware, software, information processing, and mechanical as well as electrical components (sensors, actuators, microcomputers, buses, power supplies, etc.) [Buj04], [Ise02]. Redundancy can be classified as physi$\mathrm{cal} / \mathrm{space}$ or analytical:

Space redundancy, also referred to as hardware redundancy, includes one or more extra hardware elements besides the original element. These are often connected in parallel by any of the following methods [Ise02], [Ham03]:

- Static redundancy: three or more modules are connected in parallel and receive the same input signal. A fault-free voter compares their output signals and decides by majority on the correct one. Figure 2.6 shows a redundant system with three identical modules (TMR, Triple Modular Redundancy). All receive the same input $\mathrm{Xi}$, and a voter determines the correct output Xo. In this type of redundancy, one fault can be masked without the usage of special error-detection methods. With $n$ redundant modules (n-1)/2 faults ( $\mathrm{n}$ is odd) can be tolerated.

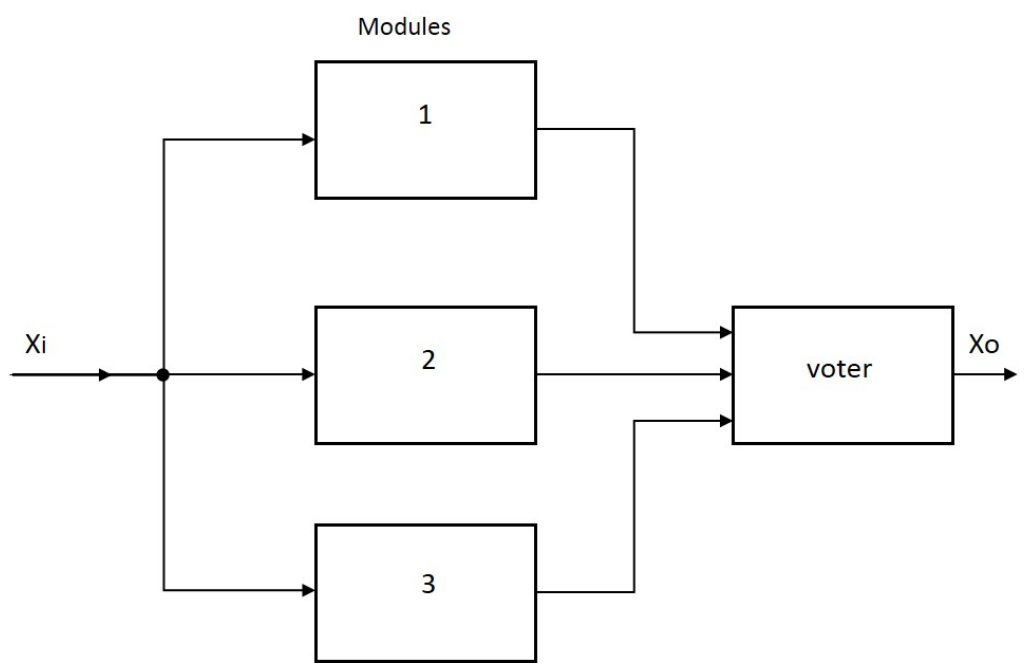

Figure 2.6: Scheme of a static Triple Modular Redundant system (TMR) [Ise02]. 
- Dynamic redundancy: this is usually implemented with two modules as shown in Figures 2.7 and 2.8. Both modules receive the same input signal Xi, one being active and the other in standby mode. If the fault-detection unit detects a faulty condition in the operational module (e.g. module 1), it is the task of the reconfiguration unit to switch to the standby module (e.g. module 2), so the system can continue to provide the output signal Xo. Fault-detection can be performed for instance, by monitoring the output signals of the modules. Figure 2.7 shows the 'hot standby' configuration, in which the standby module is continuously in operation allowing a short downtime that improves the availability of the system, but both modules are subject to the same aging. In Figure 2.8 the 'cold-standby' configuration is presented. In this case, the standby module is out of operation and hence less subjected to aging. It only becomes operational in case of a failure in the primary system.

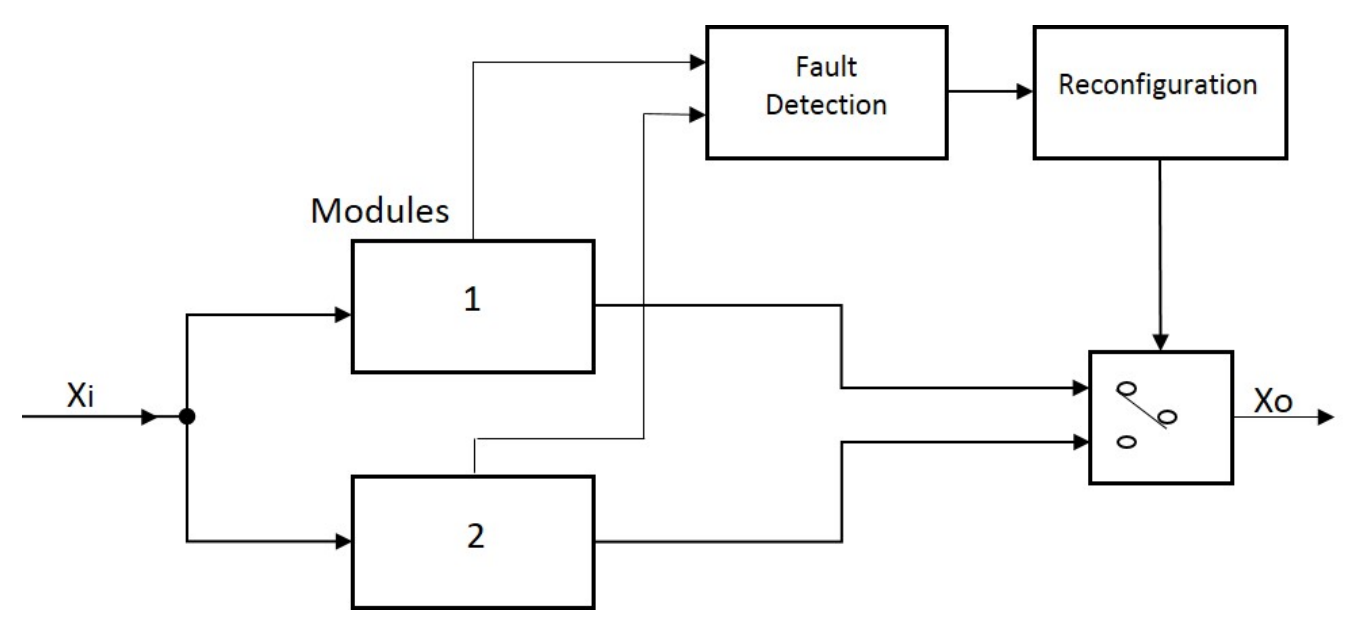

Figure 2.7: Scheme of a dynamic redundant system with 'hot standby' configuration [Ise02].

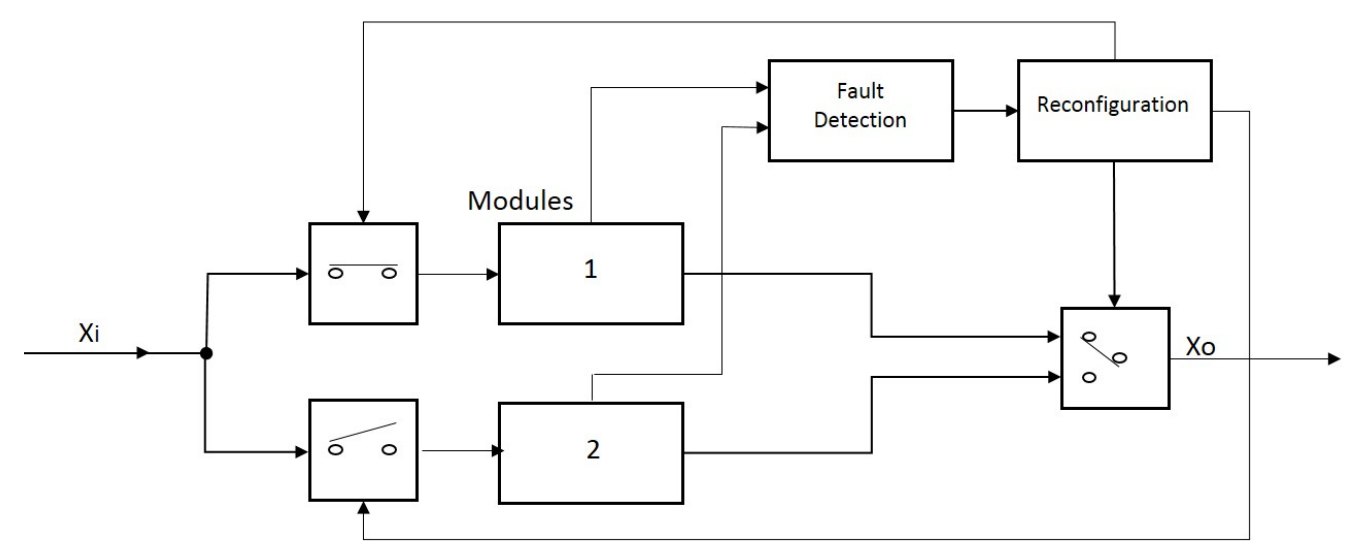

Figure 2.8: Scheme of a dynamic redundant system with 'cold standby' configuration [Ise02]. 


\subsection{Dependability}

Physical redundancy is widely used to implement fault-tolerant systems. Aeroplanes for example often use triple or even quadruple static redundant systems. In automotive applications one can find either static or dynamic redundancy. Dietmayer proposed in [Die00], a physical redundant system with two AMR sensors in which the Fault-detection is performed via plausibility checking with a value generated from the anti-parallel output curves of the sensors. Under the fault-free condition, the sum of both output values is always $95 \%$ of the power supply regardless of the actual angle. In [Inf14] a dual-sensor package is presented that includes two AMR sensors for physical redundancy. Among the disadvantages of physical redundancy are high costs, increased power consumption and weight. Furthermore, it cannot tolerate common-mode faults, or a single-point-of-failure. A single-point-of-failure could occur in the voter in the case of static redundancy or in the fault-detection module in dynamic redundancy.

In analytical redundancy, the missing information in case of a faulty condition is determined with either other hardware components or other internal values of the system. Therefore no extra hardware is required. Figure 2.9 shows a scheme of an analytical redundant system. A mathematical model of the system is often used together with some estimation techniques for fault detection, isolation and reconfiguration. This type of redundancy has been investigated to reduce redundant hardware decreasing overall cost, but still improving the system dependability [Anw07], [Gao15].

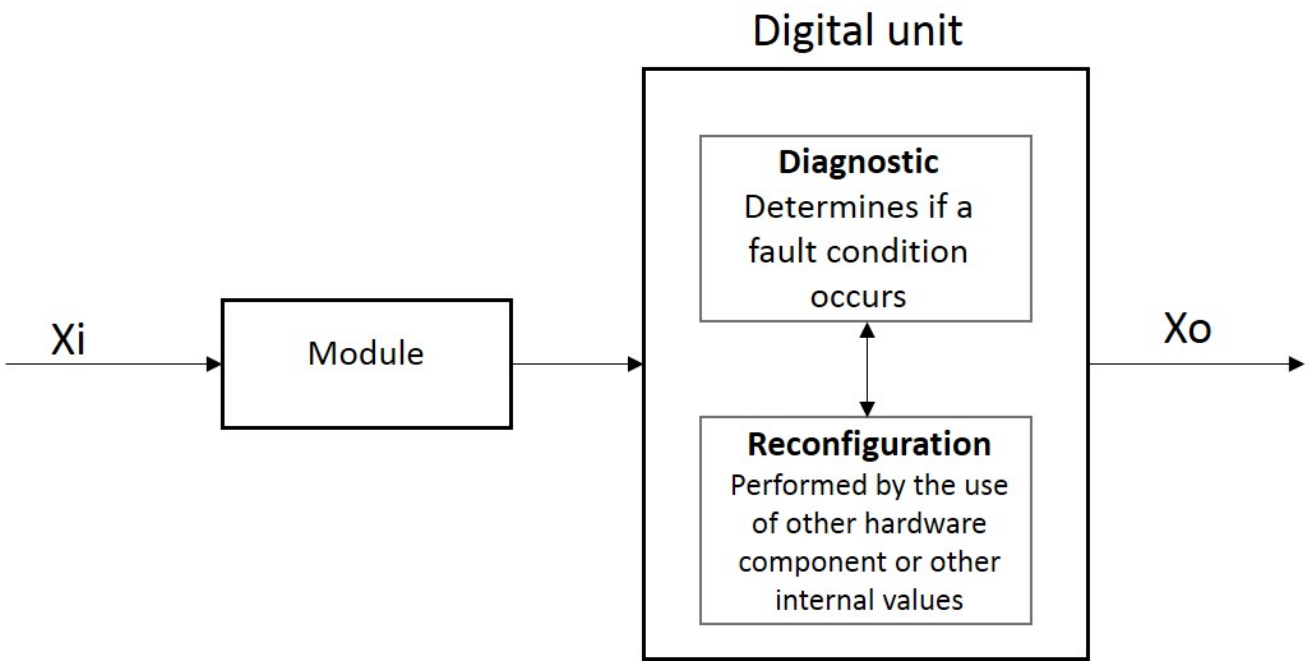

Figure 2.9: Scheme of an analytical redundant system.

In [Anw07], an analytical redundancy methodology is proposed to implement fault-tolerance in a steer-by-wire (SBW) system. Based on a steering-system model, an observer is designed to determine the road-wheel angle (RWA) from the current measurement of the road-wheel actuator. The observed value of the RWA is subsequently used as the reading of an analytical sensor, which replaces one of the three redundant hardware sensors traditionally used. The objective is to reduce the 
total number of redundant angle sensors without sacrificing the overall safety and performance of the system.

A redundant diversified steering-angle sensor system is presented by Dilge in [Dil03]. The steering angle is determined from the output of two (AMR) sensors. The fault-tolerance is achieved by adding two additional optical sensors, which also allow diversification through a different sensing principle. The four sensors s1, s2, s3 and s4 form six pairs of sensing elements p12, p13, p14, p23, p24 and p34. Therefore, the system is capable of tolerating failures in two sensors.

\subsection{Dependability requirements in automotive ap- plications}

Dependability as well as the accuracy requirements demanded by X-by-wire systems are steadily increasing, especially with the current trend of autonomous cars. For safety-critical systems, the reliability goal is to match the failure rate $(\lambda)$ of $1.10^{-9}$ per hour demanded in aviation [Ham03]. With regard to safety, it is considered that the systems used in safety-critical applications should satisfy the ASIL level D specified in the standard ISO 26262, which indicates a failure rate smaller than $1.10^{-8}$ per hour, as shown in Table 2.2 [Sin11]. Concerning maintainability, it is expected that despite the aging effects, electronic components keep their performances within the tolerance band permitted. In addition, automotive electronic systems must also strive for delivering high availability tolerating unexpected failures. This is especially important in safety-critical systems with no safe-state to break the failure-hazard sequence [Kal05].

The development of a dependable X-by-wire system is much more challenging if compared to other safety-critical applications, due to the specific requirements of the automotive field [Buj04]. X-by-wire systems must operate under harsh environmental conditions, such as temperatures from $-40{ }^{\circ} \mathrm{C}$ to $175^{\circ} \mathrm{C}$, temperature shock, vibration and moisture. The service lifetime expected for a vehicle is 15 years. During this time it is considered that the vehicle operates in cycles in which the automotive electronics experience a temperature profile that can be defined in three phases, as shown in Figure 2.10.

In the first phase, the car engine is at rest and hence the temperature is equal to the ambient temperature (T1). Then, the engine is turned on and the temperature increases up to the maximum operating value (T2) through the ramp up in phase I of the profile. In phase II, the car continues to be in operation experiencing high temperatures that can fluctuate over time. Finally, in phase III, the car engine is put to rest again going back to ambient temperature following a ramp down [Geo10]. Mechanical fatigue is considered to be the primary long-term failure mechanism for electronic components, which in principle can not be avoided. This originates from the different thermal expansion coefficients of the materials from which the components are made and the thermal stress from temperature cycling [ZVE13], [Lar03]. 


\subsection{Conclusions}

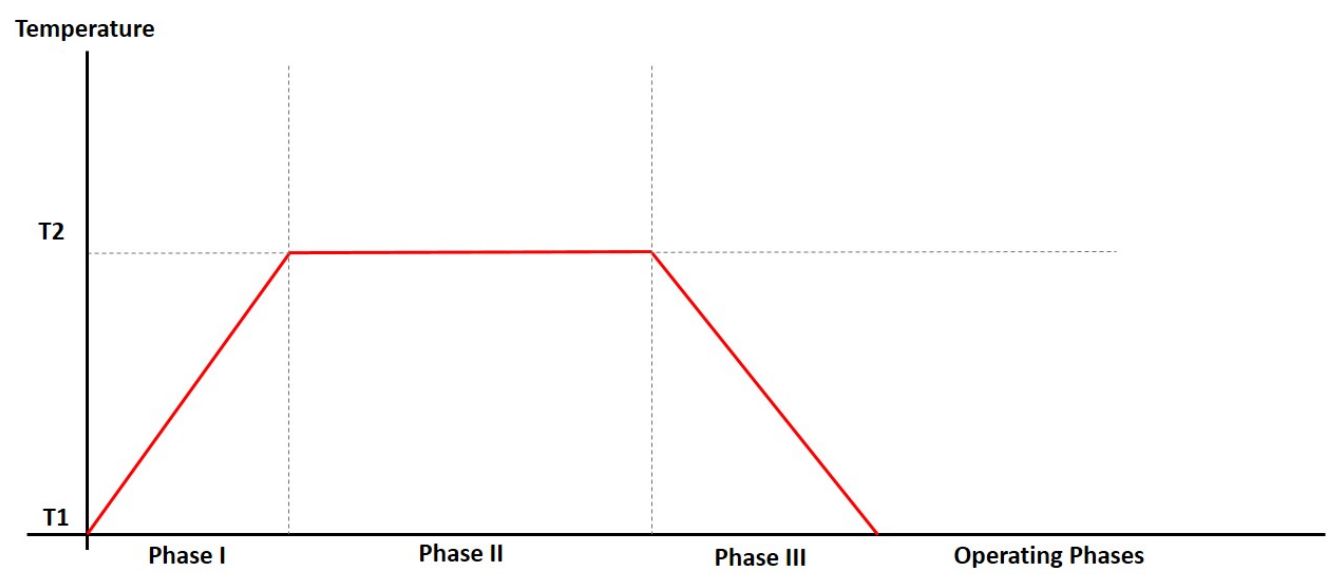

Figure 2.10: Temperature profile experienced by automotive electronic components. Phase I and III represent the transition from ambient temperature (T1) when the engine is at rest to operating temperature (T2) when the engine is on, and vice-versa. Phase two indicates the operating condition [Geo10].

Furthermore, cars are produced at a large scale and hence need to satisfy all mass-production constraints, such as low costs, system modularity and feasibility. Implementation of a new technology in the automotive industry should be compatible with the existing system to make it feasible. An X-by-wire system should be fault-tolerant, without a single-point-of-failure to guarantee the safety of the passengers as well as the cars and the environment. Fault-tolerant sensors should be fail-operational (FO) for one sensor fault [Ise02].

\subsection{Conclusions}

In this chapter, the background of AMR sensors has been presented. These sensors are widely used in automotive applications for angle measurements. However, the accuracy of the calculated angle can be affected by the following undesired parameters included in the sensor outputs: offset voltage, amplitude imbalance, and additional harmonics. Until now, the offset voltage is the parameter mainly compensated because it is the largest source of angle error. The compensation factors are calculated in the factory at the start of the sensor's life, but they are not updated during its lifetime. Although it is known that the offset voltage drifts due to wearing and aging effects, it remains within the tolerance band currently permitted. Therefore, the majority of the research has been focussed on proposing compensation methods suitable to be used in factory conditions, as detailed before.

Isler stated in [Isl10] that the drift of the offset voltage over a thousand hours at high temperature should be in the range of some $\mu \mathrm{V} / \mathrm{V}$ to guarantee an accuracy of better than $1^{\circ}$ over the sensor lifetime. However, this tolerance band will become increasingly narrow (few $\mu \mathrm{V} / \mathrm{V}$ ) as the dependability and accuracy requirements in automotive applications are continually increasing. Nevertheless, it is not yet 
clear what is the best methodology to perform aging compensation of the offset voltage or the other two undesired parameters in the case it is required. To address this question, it is important to improve the understanding of the aging effects on AMR sensors, which can be achieved by the development of aging tests focussed on studying the performance of the sensor over time.

In order to guaranty that AMR sensors for angle measurements will satisfy the new requirements demanded by automotive applications, it is also necessary to embrace strategies to guaranty the sensor performance over time. Among these strategies self-X properties and fault-tolerance are included, which have been explained in more details as they have been used in this research. 


\section{References}

[Abd06] M. Abd-El-Barr. Design and Analysis of Reliable and Fault-Tolerant Computer Systems, 2006.

[Anw07] S. Anwar and L. Chen. "An Analytical Redundancy-Based Fault Detection and Isolation Algorithm for a Road-Wheel Control Subsystem in a Steer-By-Wire System". In IEEE Transactions on Vehicular Technology, volume 56(5), pp. 2859-2869, Sept 2007.

[Bar08] A. Bartos and M. Armin. "An introduction to magnetoresistive sensors". Technical paper, MEAS Deutschland GmbH measurement specialities, Germany, 2008.

[Bat07] F. Battini, M. Tonarelli, and L. Fanucci. "Experiencing with AMR sensor conditioning in automotive field". In $2007 \mathrm{PhD}$ Research in Microelectronics and Electronics Conference, pp. 205-208, July 2007.

[Buj04] G. Buja, S. Castellan, R. Menis, and A. Zuccollo. "Dependability of safety-critical systems". In IEEE International Conference on Industrial Technology, volume 3, pp. 1561-1566, Dec 2004.

[Car98] M. J. Caruso, C. H. Smith, T. Bratland, and R. Schneider. "A New Perspective on Magnetic Field Sensing". volume 15(12), pp. 34-36, 1998.

[Die00] K. Dietmayer and M. Weser. "Contactless Angle Measurement using KMZ41 and UZZ9000". Application note an00023, Philips Semiconductors, Germany, 2000.

[Die01] K. C. Dietmayer. "Integrated online diagnosis for AMR-based angular measurement systems". In Sensors and Actuators A: Physical, volume 91(1-2), pp. 12-15, 2001.

[Dil03] E. Dilge, M. Gulbins, T. Ohnesorge, and B. Straube. "On a redundant diversified steering angle sensor". In 9th IEEE On-Line Testing Symposium (IOLTS), pp. 191-196, July 2003.

[Fel04] A. Felscher. "Programmable angle sensor KMA200. Aplication note". Technical report, Philips Semiconductors, 2004.

[Gao15] Z. Gao, C. Cecati, and S. X. Ding. "A Survey of Fault Diagnosis and Fault-Tolerant Techniques Part I: Fault Diagnosis With Model-Based and Signal-Based Approaches". In IEEE Transactions on Industrial Electronics, volume 62(6), pp. 3757-3767, June 2015.

[Geo10] E. George. Thermal cycling reliability of lead-free solders (SAC305 and SN3.5AG) for high temperature applications. Master's thesis, University of Maryland, 2010. 
[Ham03] R. Hammett and P. Babcock. "Achieving 10-9 Dependability with Driveby-Wire Systems". SAE Technical Paper 2003-01-1290, pp. 157-170, March 2003.

[Hau00] H. Hauser, G. Stangl, and W. Fallmann. "Magnetoresistive sensors". In Preparation, Properties, and Applications of Thin Ferromagnetic Films workshop, pp. 15-27, June 2000.

[Ibe03] I. E. T. Iben. "Head Reliability of AMR Sensors Based on Thermal Stress Tests". In IBM J. Res. Dev., volume 47(4), pp. 415-428, July 2003.

[Inf14] Infineon. "Dual-Sensor Package.Two Sensors in One SMD Packge". Product brief, Infineon Technologies AG, Germany, 2014.

[Ise02] R. Isermann, R. Schwarz, and S. Stolzl. "Fault-tolerant drive-by-wire systems". In IEEE Control Systems, volume 22(5), pp. 64-81, Oct 2002.

[Isl10] M. Isler, B. Christoffer, and G. Schoer. "Optimisation of surface passivation for highly reliable angular AMR sensors". Physica status solidi (c), volume 7(2), pp. 436-439, 2010.

[Joh11a] M. Johar, R. Freier, and A. Koenig. "Adding self-x capabilities to AMR sensors as a first step towards dependable embedded systems". In Proceedings of the Ninth Workshop on Intelligent Solutions in Embedded Systems (WISES), pp. 41-46, July 2011.

[Joh11b] M. Johar and A. Koenig. "Case Study of an Intelligent AMR Sensor System with Self-x Properties". In Soft Computing in Industrial Applications. Springer, 2011.

[Kal05] D. Kalinsky. "Architecture of safety-critical systems". In EE Times-India, Sep 2005.

[Ker10] H. G. Kerkhoff and J. Wan. "Dependable digitally-assisted mixed-signal IPs based on integrated self-test and self-calibration". In 16th IEEE International Mixed-Signals, Sensors and Systems Test Workshop (IMS3TW), pp. 1-6, June 2010.

[Kha11] M. A. Khan and H. G. Kerkhoff. "A system-level platform for dependability enhancement and its analysis for mixed-signal SoCs". In 14th IEEE International Symposium on Design and Diagnostics of Electronic Circuits and Systems (DDECS), pp. 17-22, April 2011.

[Kha14] M. A. Khan. On improving dependability of analog and mixed-signal SoCs: a system-level approach. Ph.D. thesis, University of Twente, Enschede, Nov 2014 .

[Lar03] O. Larses. "Modern Automotive Electronics from an OEM perspective". Technical report, Royal Institute of Technology, KTH, Sweden, 2003. 


\section{References}

[Lar14] J. Lara and A. Chandra. "Position error compensation in quadrature analog magnetic encoders through an iterative optimization algorithm". In 40th Annual Conference of the IEEE Industrial Electronics Society (IECON), pp. 3043-3048, Oct 2014.

[Lay08] G. Lay. Fault Tolerant Position Control of SM-PMSM In an ElectroMechanical Brake Actuator Against AMR Angle Sensor Failure. Ph.D. thesis, Technical University of Berlin, Berlin, Oct 2008.

[Len06] J. Lenz and S. Edelstein. "Magnetic sensors and their applications". In IEEE Sensors Journal, volume 6(3), pp. 631-649, June 2006.

[Lin11] Q. Lin, T. Li, and Z.Zhou. "Error analysis and compensation of the orthogonal magnetic encoder". In First international conference on instrumentation, measurement, computer, comunication and control, pp. 11-14, Oct 2011.

[Man01] A. Manzone, A. Pincetti, and D. D. Costantini. "Fault tolerant automotive systems: an overview". In Proceedings Seventh International On-Line Testing Workshop, pp. 117-121, 2001.

[Mut04] M. Muth. "Method for offset compensation of a magnetoresistive position or angular position measuring system", Feb 2004. US Patent 6,686,733.

[Phi00] Philips. "General magnetoresistive sensors for magnetic field measurement". Technical report, Philips Semiconductors, 2000.

[Pol95] S. Poledna. "Fault tolerance in safety critical automotive applications: cost of agreement as a limiting factor". In Twenty-Fifth International Symposium on Fault-Tolerant Computing (FTCS), pp. 73-82, June 1995.

[Rud12] R. Rudolf, R. Wilcock, and P. R. Wilson. "Reliability improvement and online calibration of ICs using configurable analogue transistors". In IEEE Aerospace Conference, pp. 1-8, March 2012.

[Sch05] H. Schmeck. "Organic computing - a new vision for distributed embedded systems". In Eighth IEEE International Symposium on Object-Oriented Real-Time Distributed Computing (ISORC), pp. 201-203, May 2005.

[Sin11] P. Sinha. "Architectural design and reliability analysis of a fail-operational brake-by-wire system from ISO 26262 perspectives". In Reliability Engineering and System Safety, volume 96(10), pp. 1349-1359, 2011.

[Tre01] C. Treutler. "Magnetic sensors for automotive applications". In Third European Conference on Magnetic Sensors and Actuators., volume 91(1-2), pp. 2-6, 2001.

[Vop13] M. Vopalensky and A. Platil. "Temperature Drift of Offset and Sensitivity in Full-Bridge Magnetoresistive Sensors". In IEEE Transactions on Magnetics, volume 49(1), pp. 136-139, Jan 2013. 
[Waf01] E. Waffenschmidt. "Method for the offset calibration of a magnetoresistive angle sensor including at least one wheatstone bridge". US Patent 6,304,074, Oct 2001.

[Zhu16] H. Zhu, Y. Ge, and B. Jiang. "Modified CORDIC algorithm for computation of arctangent with variable iterations". In IEEE 13th International Conference on Signal Processing (ICSP), pp. 261-264, Nov 2016.

[Zie11] V. Zieren and V. Van. "Magnetic field angular sensor and sensing method". European Patent App. EP20,100,152,598, Aug 2011.

[Zor17] Y. Zorian. "Automotive reliability and test strategies". Tutorial ITC-Asia, Sep 2017.

[ZVE13] ZVEI (Zentralverband Elektrotechnik- und Elektronikindustrie). "Handbook for Robustness Validation of Automotive Electrical/Electronic modules". June 2013. 


\title{
Chapter 3
}

\section{Characterization of AMR sensors}

\begin{abstract}
In this chapter, the parameters used to characterize AMR sensors are presented. Next two aging experiments based on Accelerated Degradation Test (ADT) that have been performed are explained in detail. This includes the setup used to age the sensors, the setup for measurements, test procedures and results. Finally, an analysis of the aging effects on the performance of AMR sensors is presented.
\end{abstract}

\subsection{Introduction}

The characterization of a device is aimed to determine its fundamental electrical and physical characteristics based on the analysis of experimental data. This process is usually performed during the development of a new device to ensure it meets the requirements defined for it [AEC13]. However, characterization can also be useful to study the wearing and aging effects on the device performance over time. Then, it is possible to determine if the device can and will continue to perform its targeted functions according to the product definition. In this case, the characterization should be developed together with an aging test.

Our research studies the aging effects on the performance of AMR sensors used for angle measurements. The goal is to determine whether the device will satisfy the continuously increasing requirements for automotive applications. An AMR sensor can be characterized based on the following parameters: bridge resistances, offset voltage, the amplitude of the sinusoidal signals at the sensor outputs, and the angle error. A set of aging tests to study the performance degradation of AMR sensors has been developed.

The sensor used in this research is the KMZ49 for angle measurements from NXP [NXP11]. For this sensor, it is required to apply a magnetic field of at least 314 Gauss to bring it in the saturation state. The main parameters of the sensor are listed in Table 3.1 that includes the range allowed for the DC supply voltage, peak

Parts of this chapter have been presented at the 20th and 21th IEEE International Mixed-Signals Testing Workshop (IMSTW) [Zam15], [Zam16]. 
voltage at the sensor outputs, offset voltage at the sensor outputs which is presented per Volt of the power supply, bridge resistance, amplitude synchronism between the outputs and the temperature coefficients (TC). These values are specified based on the conditions stated at the bottom of the Table. The pinning information of the sensor is described in Figure 3.1.

Table 3.1: Main parameters of the AMR sensor KMZ49 [NXP11].

\begin{tabular}{|l|c|c|c|c|c|}
\hline Parameter & Symbol & Min & Typ & Max & Unit \\
\hline Supply Voltage & $V_{c c}$ & - & 5 & 9 & $\mathrm{~V}$ \\
Peak Voltage & $V_{M}$ & 60 & 67 & 75 & $\mathrm{mV}$ \\
Offset voltage/supply voltage & $V_{\text {offset }}$ & -2 & - & +2 & $\mathrm{mV} / \mathrm{V}$ \\
Bridge resistance [1] & $R_{\text {bridge }}$ & 2.7 & 3.2 & 3.7 & $\mathrm{k} \Omega$ \\
Offset Temperature coefficient(TC) & $\mathrm{TC} V_{\text {offset }}$ & -2 & - & +2 & $(\mu \mathrm{V} / \mathrm{V}) / \mathrm{K}$ \\
Peak voltage TC & $\mathrm{TCV} V_{M}$ & -0.3 & -0.36 & -0.42 & $\% / \mathrm{K}$ \\
Bridge resistance TC & $\mathrm{TC} R_{\text {bridge }}$ & 0.24 & 0.26 & 0.29 & $\% / \mathrm{K}$ \\
Amplitude synchronism & $\mathrm{K}$ & 99.5 & 100 & 100.5 & $\%$ \\
\hline
\end{tabular}

[1] bridge resistance between pin 4 to pin 8 , pin 3 to pin 7 , pin 1 to pin 5 , pin 2 to pin 6 .

$$
T_{a m b}=25^{\circ} \mathrm{C} ; H_{\text {ext }}=314 \text { Gauss } ; V_{c c}=5 \mathrm{~V}
$$

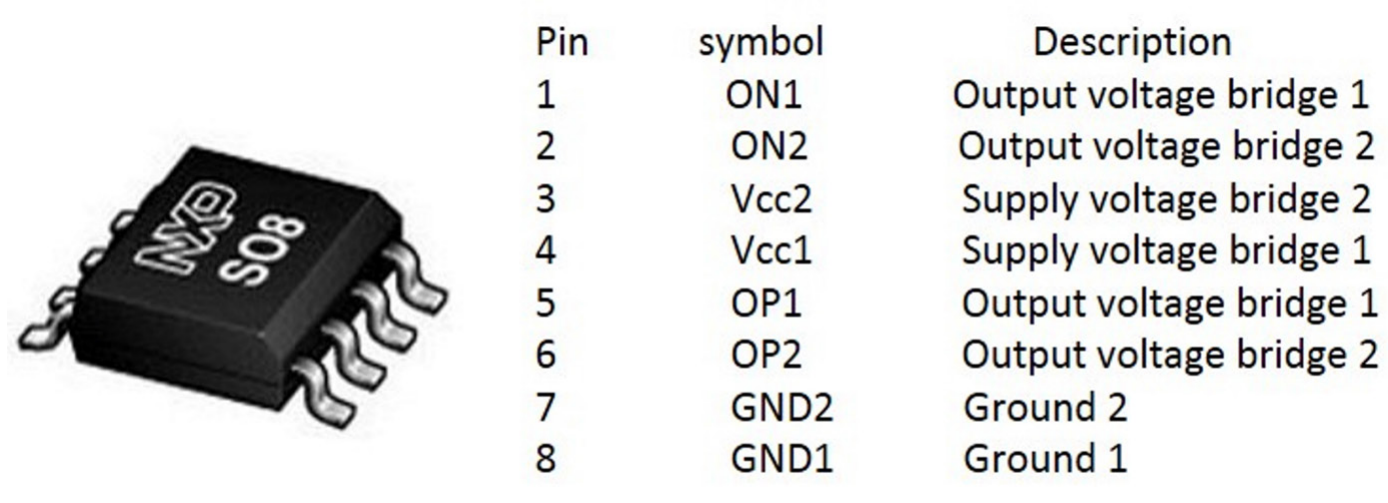

Figure 3.1: Pinning information of the AMR sensor KMZ49 [NXP11].

The remainder of this chapter is organized as follows. Section 3.2 explains the procedures followed to calculate the parameters used to characterize AMR sensors, together with the characterization results of 10 AMR sensors KMZ49. Section 3.3 presents the aging tests that have been executed to study the sensor's performance over time, including setup used to age the sensors, setup to perform the required measurements, test procedures, results as well as the discussion of them. Finally, conclusions are drawn in section 3.4. 


\subsection{Parameters to characterize the AMR sensors}

This section describes the procedures that have been followed to calculate the following sensor parameters: bridge resistance, offset voltage, amplitude, amplitude imbalance and angle error. In addition, the characterization results of $10 \mathrm{AMR}$ sensors KMZ49 are included. The required measurements were carried out at room temperature at 20 angles between 0 and 180 degrees, using a power supply of $5 \mathrm{~V}$ for the sensors and a magnetic field of 400 Gauss that is sufficient to saturate the sensors used.

\subsubsection{Anisotropic Magnetoresistance}

An AMR sensor for angle measurements includes eight magnetoresistances configured into two Wheatstone bridges placed at 45 degrees to each other as shown in Figure 3.2. In the saturation state of the sensor, the resistances show a sinusoidal behaviour that depends on the angle to be measured [Fel04]. The procedure to be followed to calculate the resistance values at each angle is explained next. It has been specified for bridge two but can also be applied to bridge one.

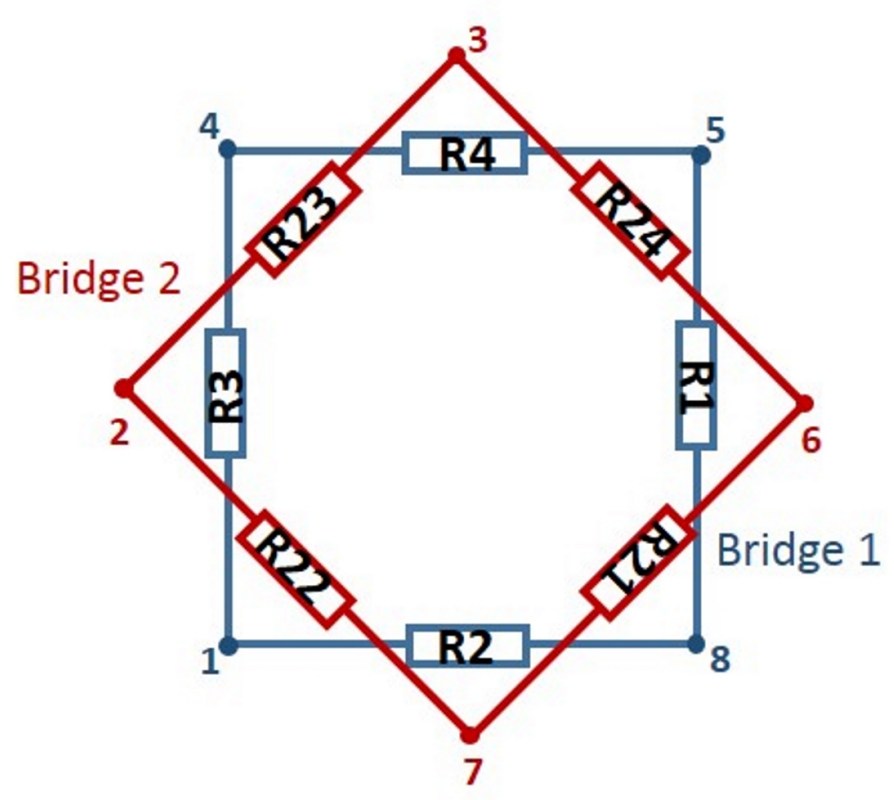

Figure 3.2: Schematic of the Wheatstone bridges in an AMR sensor in which each $\mathrm{R}$ represents a magnetoresistance. The numbers refer to the pins in Figure 3.1.

1. Several voltages should be measured with the sensor configured in two modes as shown in Figure 3.3. In normal mode, the power/output pins are configured as depicted in Figure 3.1, and inverted mode means power/output connections are inverted. 


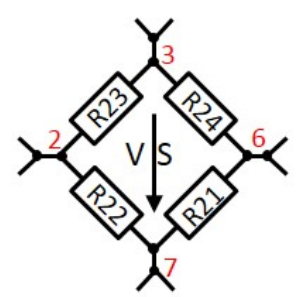

Normal

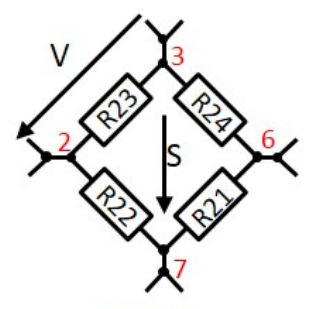

Normal

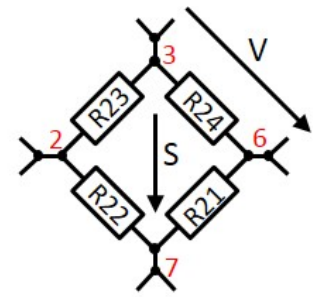

Normal

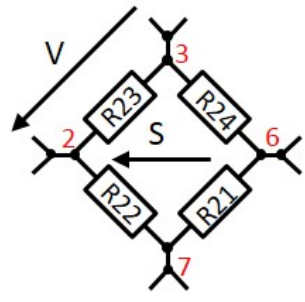

Inverted

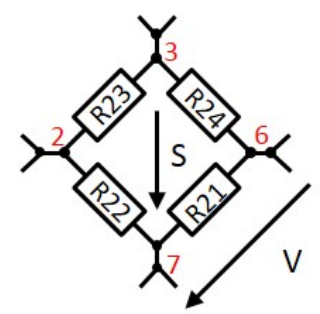

Normal

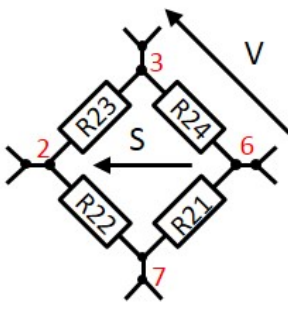

Inverted

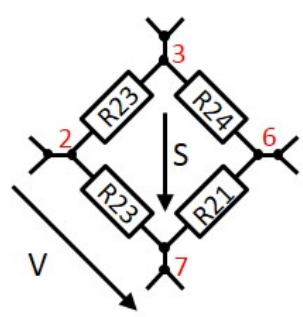

Normal
$\mathrm{V}=$ measured voltage

$\mathrm{S}=$ power supply

$\longleftarrow$ indicates polarity of the power supply and for the voltage measurement

Figure 3.3: Voltages measured to calculate the bridge resistances.

2. From the measurements, the diagonal resistance (Rdiag) of the bridge is determined with Equation (3.1). This means the resistance between the connection points 3 and 7 in Figure 3.3. Besides, the voltage relationships between the resistances are calculated with Equations (3.2), (3.3) using voltages measured in normal mode and in Equation (3.4) with voltages measured in inverted mode.

$$
\begin{aligned}
\text { Rdiag } & =\frac{V_{37}}{i_{37}} \\
A & =\frac{V_{R 21}}{V_{R 24}} \\
B & =\frac{V_{R 22}}{V_{R 23}} \\
C & =\frac{V_{R 23}}{V_{R 24}}
\end{aligned}
$$

3. The resistances values are determined by Equations (3.5),(3.6),(3.7) and (3.8).

$$
\begin{aligned}
R 21 & =A * R 24 \\
R 22 & =C * B * R 24 \\
R 23 & =C * R 24 \\
R 24 & =\frac{(1+C+C * B+A) * R d i a g}{C *(1+A) *(1+B)}
\end{aligned}
$$


As mentioned in the previous chapter, the bridge resistances can be described with Equation (3.9). In theory, the resistances should have the same values for Ro and $\triangle R o$, meaning that resistances with the same orientation in the bridge should show the same sinusoidal characteristics, as they detect the same angle between the magnetic field and the current flow. This should be the case for the resistance pairs $(\mathrm{R} 1, \mathrm{R} 3) ;(\mathrm{R} 2, \mathrm{R} 4)$ in bridge 1 and $(\mathrm{R} 21, \mathrm{R} 23)$; $(\mathrm{R} 22, \mathrm{R} 24)$ in bridge 2 as shown in Figure 3.2.

$$
R=R_{o}+\triangle R_{o} \cdot \cos (\theta)^{2}
$$

However, due to imperfections introduced during the sensor manufacturing or assembly, the actual resistances show mismatches, as presented in Figures 3.4 and 3.5 for bridge 1 and 2 of an AMR sensor KMZ49. In bridge 1, R1 and R3 show a mismatch of $0.48 \Omega$, while R2 and R4 differ $1.61 \Omega$. In bridge $2, \mathrm{R} 21$ and R23 show a mismatch of $1.85 \Omega$ and R22, R24 differ $8.32 \Omega$.

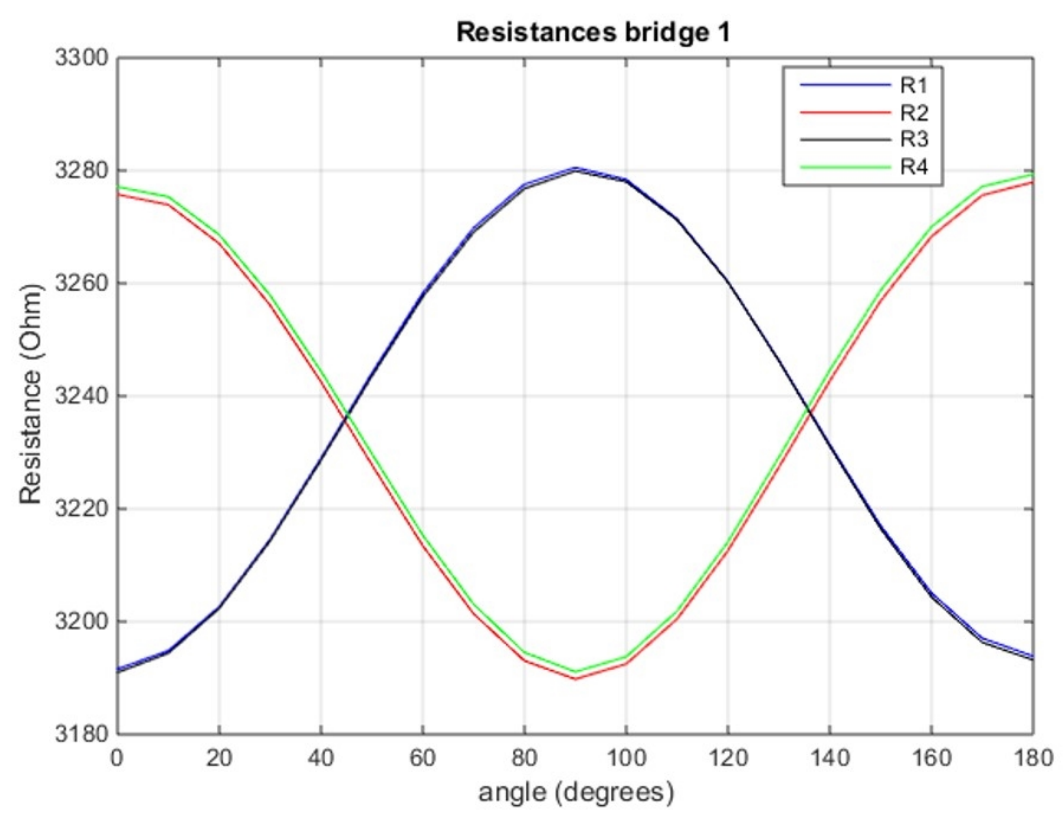

Figure 3.4: Resistance values in bridge 1 of a commercial AMR sensor KMZ49 that have been calculated from measured voltages. 


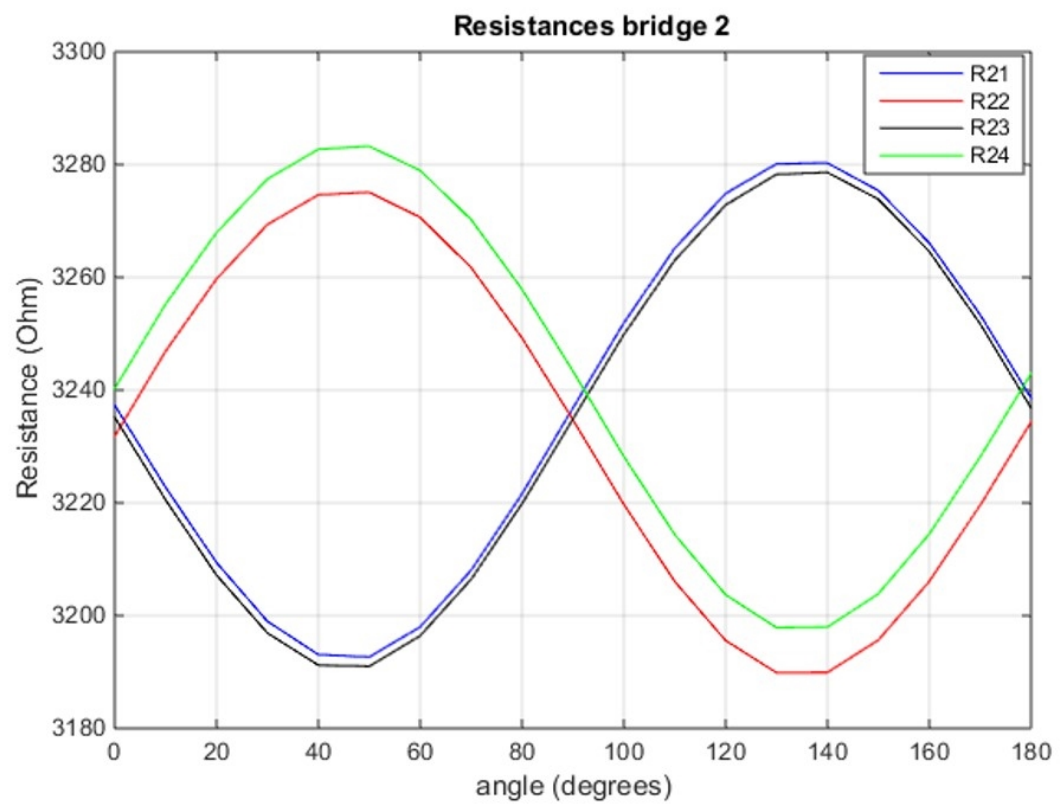

Figure 3.5: Resistance values in bridge 2 of a commercial AMR sensor KMZ49 that have been calculated from measured voltages.

Figure 3.6 shows the arithmetic mean value of each resistance pair in ten commercial sensors. The values are between $3.18 \mathrm{~K} \Omega$ and $3.30 \mathrm{~K} \Omega$, which are within the range specified by the sensor datasheet $(2.7 \mathrm{~K} \Omega-3.7 \mathrm{~K} \Omega)$ [NXP11]. However, all the devices present resistance mismatch, being the largest and smallest variations between the resistances of sensor 9 and sensor 8, which show standard deviations of $4 \Omega$ and $1.2 \Omega$ respectively.

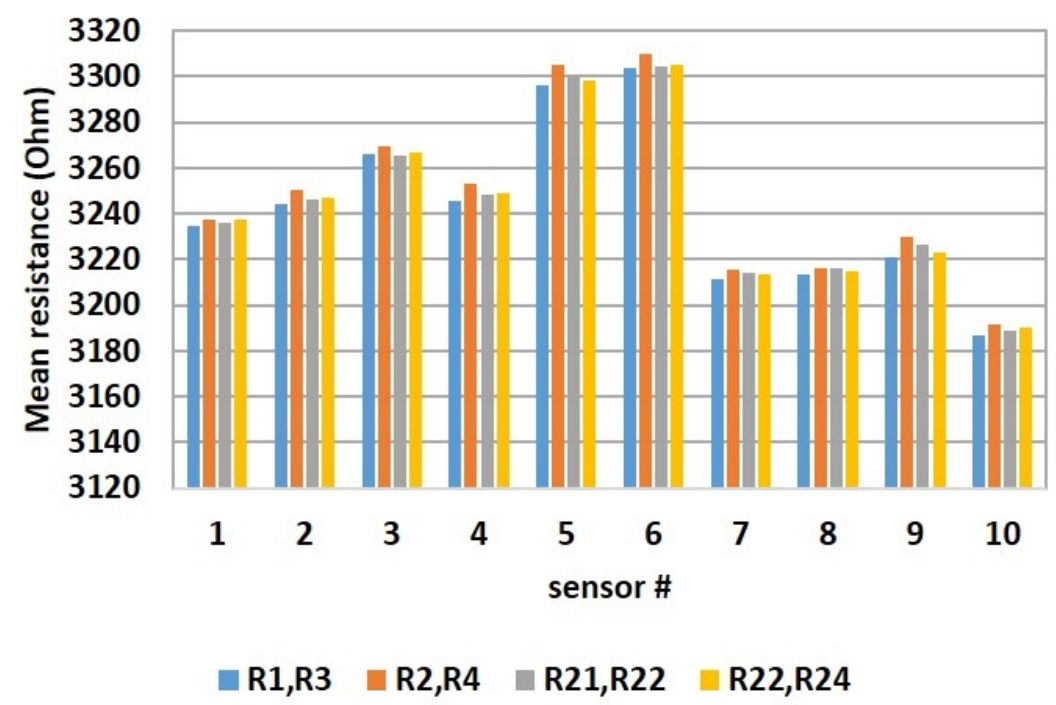

Figure 3.6: Arithmetic mean values of the resistance pairs (R1,R3), (R2,R4), (R21,R23), (R22,R24) in 10 commercial AMR sensors. 
All the sensors show bridge resistance values within the range allowed, but also all of them show a mismatch between their resistances. This brings as a consequence that all the sensor outputs include undesired characteristics, such as offset voltage, amplitude imbalance and additional harmonics, as presented below.

\subsubsection{Offset Voltage}

The offset voltage is one of the undesired parameters included in each of the sensor outputs affecting the accuracy of the calculated angle. It can be determined by a geometry method based on Lissajous curves that indicate the phase relationship between two signals on an XY coordinate axis [Ted16]. Due to the 90-degree phase difference between the two sinusoidal signals at the sensor outputs, the Lissajous curve should be a circle centred at the coordinate origin $(0,0)$ in the ideal case. The radius represents the amplitude of the sinusoidal signals and the centre the offset voltages. Therefore, the offset voltages shift the centre of the Lissajous curve, as shown in Figure 3.7.

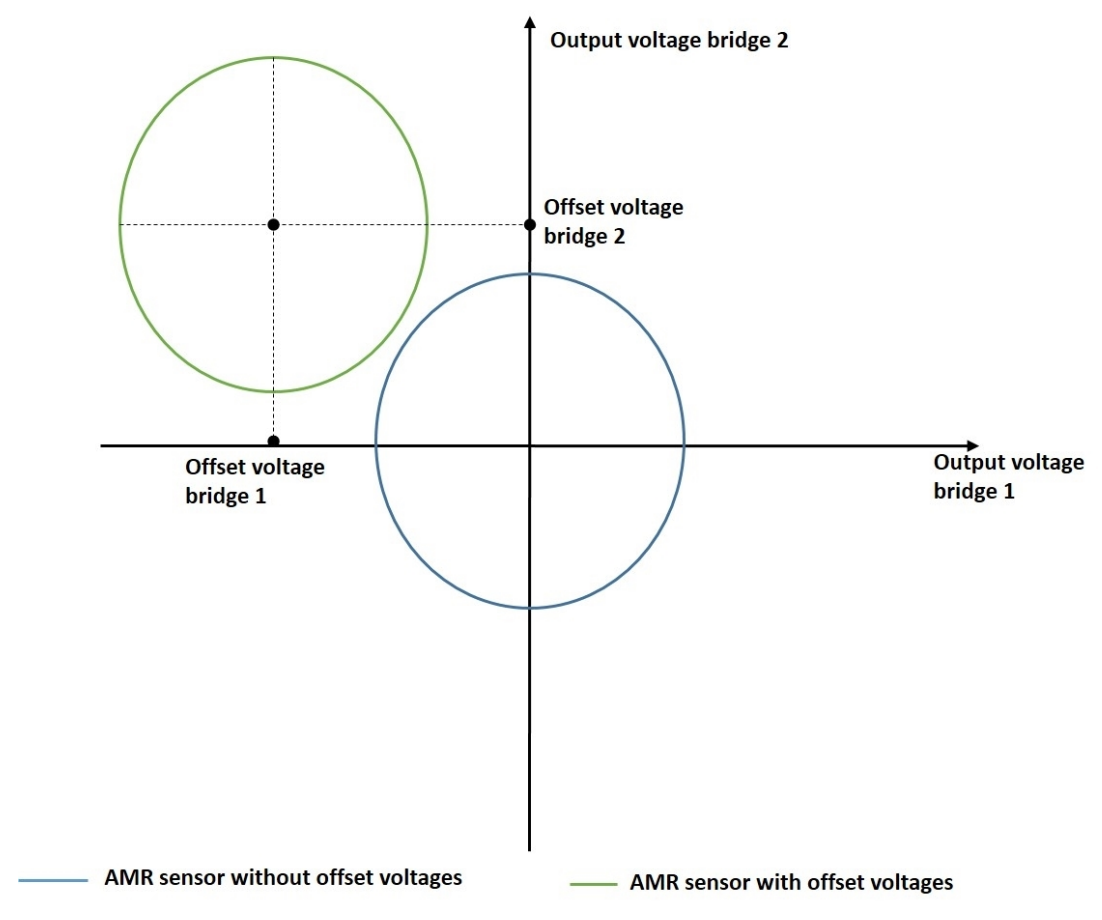

Figure 3.7: Lissajous curves of an AMR sensor with and without offset voltage.

The offset voltages of an AMR sensor can be calculated from the centre of the Lissajous curve constructed with voltages measured at several angles in a cycle of the sinusoidal signals at the bridge outputs. The offset voltage is usually specified as the ratio between the offset voltage and the voltage used to power the bridges, being indicated by the offset value per Volt of the power supply. Figure 3.8 shows the offset voltages of the ten commercial sensors that have been characterized by us, 
in which all the values are within the range specified by the datasheet [NXP11]. It shows that sensor 9 has the largest offset voltages with $657 \mu \mathrm{V} / \mathrm{V}$ and $-561 \mu \mathrm{V} / \mathrm{V}$ for bridge 1 and 2 respectively.

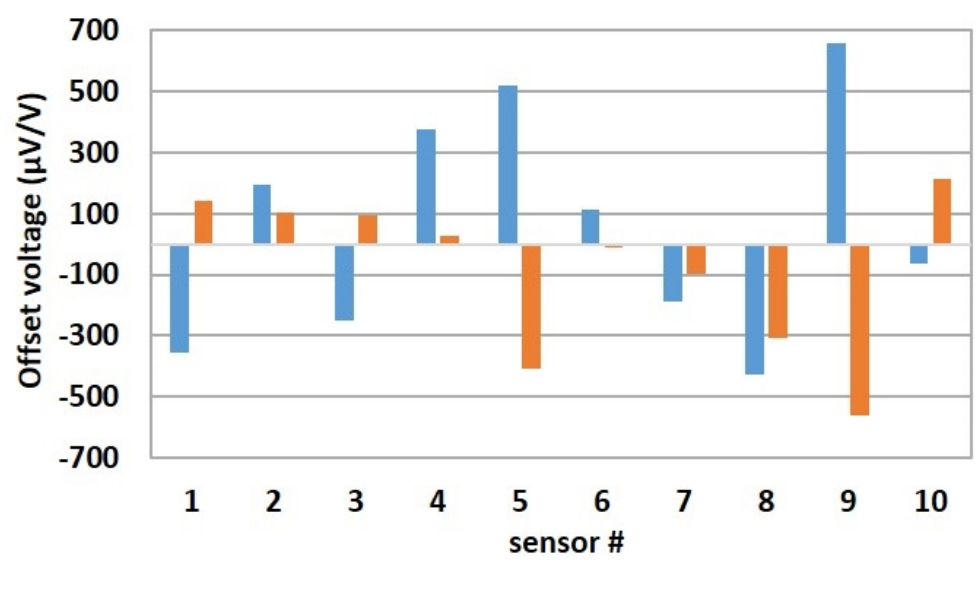

Bridge 1 Bridge 2

Figure 3.8: Offset voltages of the ten characterized commercial AMR sensors.

The results confirm that all the sensors show offset voltages in both bridges, which can be both positive, negative or with signed inverted (one bridge positive and one bridge negative). The values depend on the specific characteristics of each sensor.

\subsubsection{Amplitude of the sinusoidal signals at the outputs of AMR sensors}

The amplitude of the sinusoidal signals at the sensor outputs can also be calculated from the Lissajous curves. However, because the signal amplitudes at the bridge outputs are not equal in practice, then instead of a circle, the Lissajous curve tends to be an ellipse. The radius on the $x$ and $y$ axes represent the amplitude of the sinusoidal signals in bridge one and two respectively. The centre of the ellipse still represents the offset voltages.

In our research a fit ellipse formula has been used in MATLAB to calculate the offset voltages as well as the amplitude of the bridge outputs of the ten characterized AMR sensors as shown in Figures 3.8 and 3.9 respectively. The sensors show amplitudes between $67.58 \mathrm{mV}$ and $68.36 \mathrm{mV}$, which are within the range specified by the datasheet of $60 \mathrm{mV}$ to $75 \mathrm{mV}$ in the case the sensor is powered with $5 \mathrm{~V}$. However, all the sensors show amplitude imbalance between their bridge outputs, as indicated by the difference between the blue and orange bars in each sensor. This can be verified using the factor called amplitude synchronism $(k)$ presented in Equation 
(3.10) [NXP11].

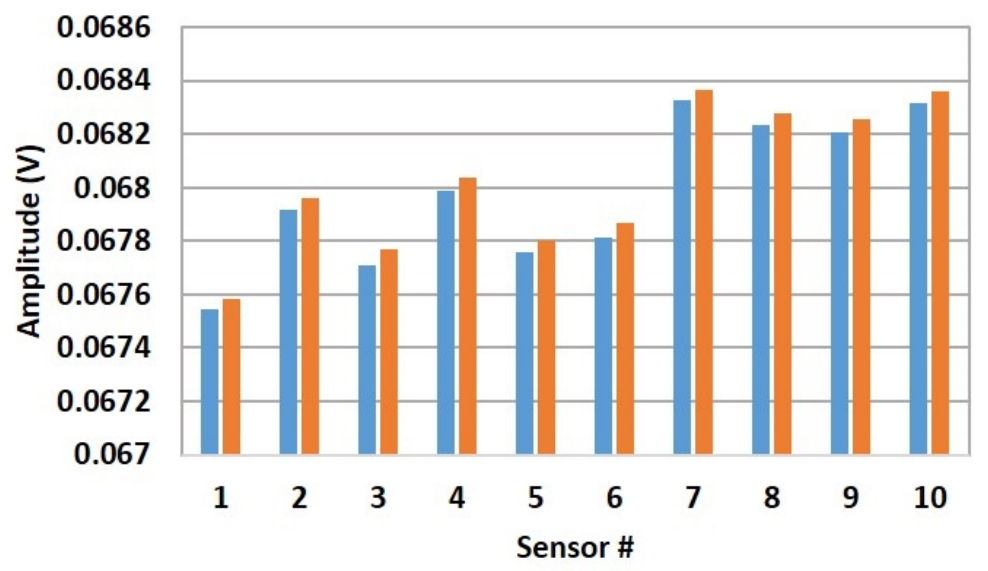

Bridge 1 Bridge 2

Figure 3.9: Amplitudes of the bridge outputs in the ten characterized commercial AMR sensors.

$$
k=\frac{\text { amplitude } e_{\text {outputbrige } 1}}{\text { amplitude }_{\text {outputbrige } 2}} * 100 \%
$$

Figure 3.10 shows the $k$ factor for the ten characterized AMR sensors. All the values are within the range specified in the datasheet between $99.5 \%-100.5 \%$. Sensor 1 displays the best result as its $\mathrm{k}$ factor is most close to $100 \%$, meaning less amplitude imbalance between sinusoidal signals at the sensor outputs.

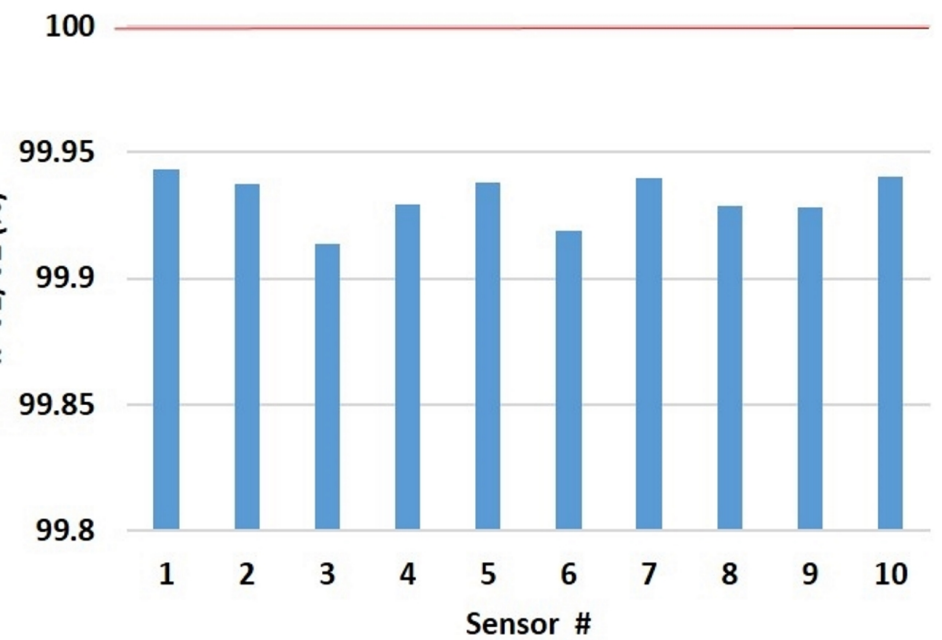

Figure 3.10: Amplitude imbalance in the ten characterized commercial AMR sensors. 


\subsubsection{Angle error}

The undesired characteristics included in the output signals of an AMR sensor affect the accuracy of the calculated angles. Therefore the offset voltage, the amplitude imbalance and the additional harmonics are considered sources of the angle error, which show sinusoidal behaviours as presented in Table 3.2. Another source of the angle error refers to imperfections in the measurement setup, such as mechanical misalignment between the sensor and the magnet generating the magnetic field or an insufficient magnetic field for the sensor being in the saturation state. However, these last issues can be reduced by improving the setup in which the sensor is used. In this research, we focussed on the angle errors due to the undesired parameters included in the sensor outputs because of its intrinsic characteristics.

Table 3.2: Expressions of the angle error for the error sources present in AMR sensors [Lar14].

\begin{tabular}{|l|c|}
\hline error source & angle error expression \\
\hline Offset voltage & $\Delta O \sin (\alpha)$ \\
Amplitude imbalance & $\Delta A \sin (2 \alpha)$ \\
Harmonic distortion & $-\sqrt{2} K_{o} \cos (\alpha+\pi / 4)-\sum_{n=2}^{\infty} K_{n} \sin [(n-1) \alpha]$ \\
\hline
\end{tabular}

- $\alpha$ is equal to $2 \theta$ which represents the angle detected by the sensor.

- $\Delta O$ is related to the ratio of the offset voltages in the sensor outputs.

- $\Delta A$ is related to the ratio of amplitude imbalance between the sensor outputs.

- $K_{o}, K_{n}$ represent harmonic coefficients and $n$ is the number of the harmonic.

The angle errors can be determined by the usage of instruments such as an oscilloscope and spectrum analyzer to determine the parameters of the signals presented in Table 3.2. Another option is to subtract the angles calculated before and after compensating for the source of the angle error. In our research, the angle error due to offset voltage has been obtained by subtracting the calculated angles with the sensor outputs without any compensation (angle1) and the calculated angles with the sensor outputs after compensating for offset voltage (angle2). Then, for the angle error due to amplitude imbalance angle 2 and angle3 that represents the calculated angles with the sensor outputs with offset voltage and amplitude imbalance compensated were subtracted.

Regarding the angle error due to harmonic distortion, it was not possible to determine all the components specified in Table 3.2. A manual setup was used to set the magnetic angles in which the sensor outputs were measured for one cycle of the sinusoidal signals. Hence an analysis of the frequency components could not be performed. However, in practice the harmonics manifest as distortion in the sensor outputs in which the third distortion coefficient is a function of not only the $3^{\text {rd }}$ 
harmonic but also of the fundamental component, as shown by the equivalent trigonometric identities presented in Equations (3.11) and (3.12) [Wik15], [Gon15]. In the fundamental components, this is represented by a DC voltage present in the sensor outputs already compensated for offset voltage and amplitude imbalance [Lar14]. After compensating for this DC voltage, the sensor outputs were used to calculate angle 4 , which was subtracted from angle3 presented above to obtain the cosine signal indicated for harmonic distortion in Table 3.2.

$$
\begin{aligned}
& \sin ^{3}(\alpha)=\frac{3 \sin (\alpha)-\sin (3 \alpha)}{4} \\
& \cos ^{3}(\alpha)=\frac{3 \cos (\alpha)+\cos (3 \alpha)}{4}
\end{aligned}
$$

Of primary interest is the maximum value of each angle error, which is obtained from the maximum of each of the sinusoidal signals that represent each source of error. Figure 3.11 shows the maximum values of the angle errors in the ten characterized commercial sensors. The values are between the following ranges: due to offset voltage (0.25 - 1.85) degrees, amplitude imbalance (0.13 - 0.19) degrees, and harmonics $(0.046$ - 0.061) degrees. The offset voltage is clearly the largest contributor to maximum angle errors followed by the amplitude imbalance and finally the harmonic component.

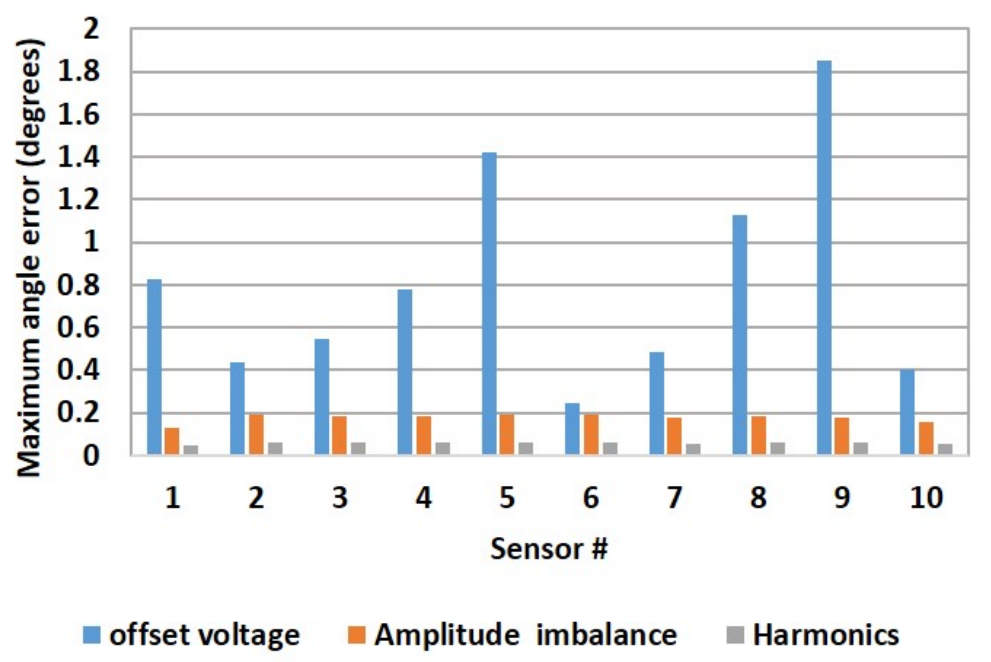

Figure 3.11: Maximum angle errors in ten commercial AMR sensors.

If one compares the results, sensor 9 shows the largest offset voltages in Figure 3.8 and the maximum angle error due to this parameter in Figure 3.11. In Figure 3.10, sensor 1 shows the closest value to $100 \%$ for the amplitude synchronism, which means less amplitude imbalance and hence less angle error for this source, as shown in Figure 3.11. Overall, the characterization results in our ten commercial target sensors 
confirm that AMR sensors show a mismatch between their resistances, and hence, undesired parameters included at the bridge output voltages affect the performance of the sensor.

\subsection{Effects of aging on AMR sensors}

AMR sensors for angle measurements are often used in automotive applications. However, as shown previously the undesirable characteristics included in the bridge output voltages affect the performance of the sensor. Until now the offset voltage is the parameter mainly compensated for under factory conditions before shipment. Although the undesirable parameters drift over time, the sensor performance remains within the tolerant band currently permitted, and therefore aging compensation is usually not applied to the sensor. To the best of our knowledge, there is little published data on the effects of aging on AMR sensors used for angle measurements.

Nevertheless, the dependability and accuracy requirements in automotive applications are continually increasing. For instance, Isler stated in [Isl10] that the drift of the offset voltage over a thousand hours at high temperature should be in the range of some tens of $\mu \mathrm{V} / \mathrm{V}$, which can be considered as less than $100 \mu \mathrm{V} / \mathrm{V}$ to guarantee an accuracy better than $1^{\circ}$ over the sensor's lifetime. However, in the future it is expected that this tolerant band for drifting will decrease to few tens of $\mu \mathrm{V} / \mathrm{V}$ (less than $50 \mu \mathrm{V} / \mathrm{V}$ ) in order to provide accuracy better than $0.1^{\circ}$ over the sensor lifetime.

Consider for example, the scenario that is shown in Figure 3.12, in which it is required that a car that drives in the middle of the lane of $3 \mathrm{~m}$ wide does not end up on the other side of the road in 500 meters. Then the maximum angle error that can be allowed in the AMR sensor used in the steering system must be less than $0.1^{\circ}$, which is especially important in autonomous cars.

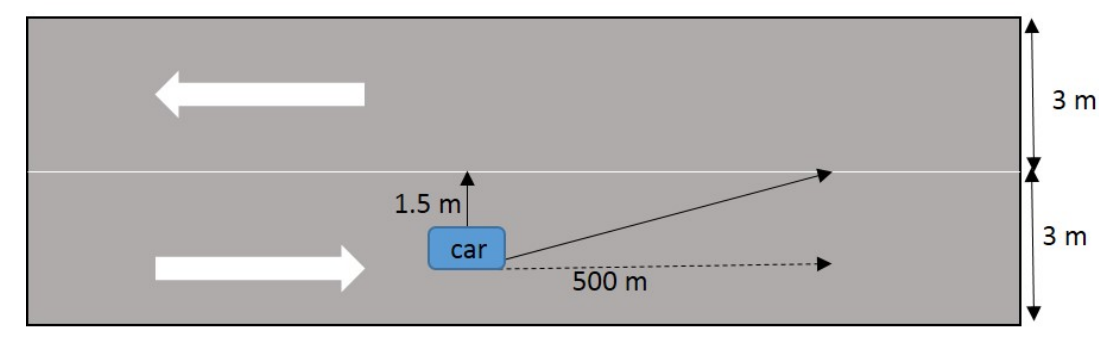

Figure 3.12: Scenario in which is considered that AMR sensors are used in the steering system of a car.

In order to determine if aging compensation will be required in AMR sensors for angle measurements, it is essential to have a better understanding of the aging effects on the sensors. Aging refers to an internal process in which the occurrence of gradual degradation brings the electronic components closer to failure [Gor09]. 
Therefore, aging tests focussed on performance degradation must be developed.

Accelerated degradation tests (ADT) represent one of the best options to study aging effects over time, in which the characteristics of accelerated and degradation tests are combined. Accelerated tests are often used to assess the lifetime of products by focusing on time-to-failure in tests that increase the stress level of parameters such as temperature, humidity or voltage [Vas08]. A traditional degradation test focusses on studying the wearing effects by submitting the product to normal operating conditions as if it is under normal use [Ber08].

ADT aims to accelerate the degradation process by submitting the product to the operational environment but under high stress regarding for example temperature, voltage or vibration [Liu10]. This test can be used for the reliability assessment of highly reliable components, which are not likely to fail in traditional life tests of reasonable length of time [Yum07]. In ADT one must take into consideration design specifications as well as the application environment. Also, it is required to consider a quantitative parameter such as a current or a voltage value which degradation over time is correlated with the performance degradation of the component [Moh10], [Liu10].

Before conducting an accelerated degradation test, a set of factors such as test stress levels, sample size, measurement frequency, and test time should be specified [Li02]. Several types of stress levels have been proposed for ADT, including constant stress (CSADT) and step stress (SSADT). In a CSADT, the devices under study are divided into several groups, and each group is exposed to certain stress conditions to collect degradation data. For SSADT, only one group is needed to conduct the experiment, in which the group may experience various stress conditions successively until the test is completed [Che16], [Wan16]. ADT is often used to assess the reliability of highly reliable products in term of characteristics such as the mean time to failure (MTTF). For this purpose, the selection of the stress level is usually based on constraints such as budget, test duration and sample size [Che16].

However, our research is focussed on the study of the performance degradation of AMR sensors over time instead of the estimation of the sensor lifetime. CSADT as well as SSADT have been used to study the effect of different stress conditions on the performance of the sensors. Regarding the operating conditions of the sensors, as mentioned in Chapter 2 (section 2.4), it has been considered that automotive electronics operates in cycles with temperature excursions between ambient air temperature (T1) and maximum operating temperature (T2). In total two aging tests have been performed. The first test focusses on the aging effects on the offset voltage and the amplitude of the sensor outputs. In the second test, also the bridge resistances and the angle error were included. Both tests will now be explained in detail, including setup used to age the sensors, setup used to perform the required measurements, test procedures and the results. 


\subsubsection{The first aging test}

\section{The aging setup}

During this first test, SSADT has been used to submit one group composed of three sensors KMZ49 to an ADT using the following stress conditions regarding temperature, magnetic field and power voltage. Temperature cycles have been used to stress the sensors. To define the temperature range, it has been taken into consideration that the datasheet of the AMR sensor KMZ49 indicates an ambient temperature of $25^{\circ} \mathrm{C}$ and maximum temperature of $150^{\circ} \mathrm{C}$ [NXP11]. Therefore, as in ADT higher stress levels should be used, temperature cycles between $30^{\circ} \mathrm{C}$ and $180^{\circ} \mathrm{C}$ have been applied. The cycling time was approximately 24 minutes (10 minutes heating, 2 minutes dwelling time and 12 minutes cooling).

Concerning the magnetic field, the coils shown in Figure 3.13 were used to generate a measured magnetic field of 400 Gauss; this is sufficiently strong to saturate the sensors located inside the furnace placed between the coils. With regard to the power supply, in normal operation the sensor is powered with $5 \mathrm{~V}$; in the case of high stress a voltage of $8.5 \mathrm{~V}$ has been used.

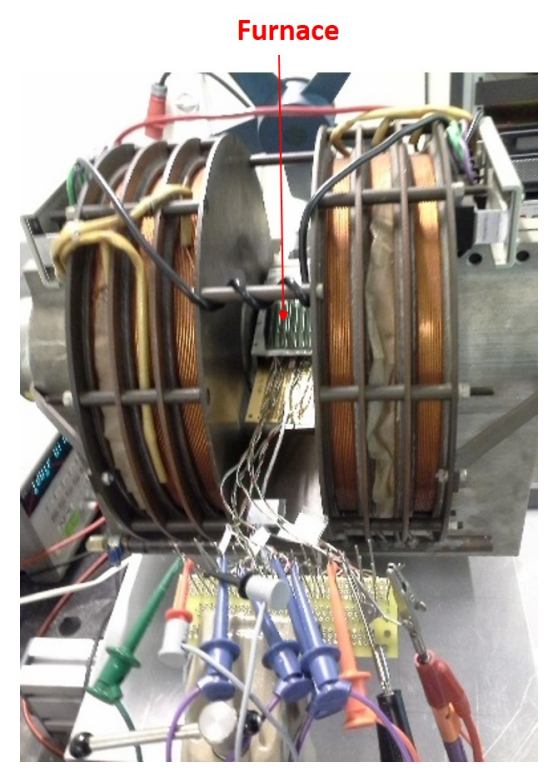

Figure 3.13: Setup used to age our AMR sensors during the first aging test.

\section{Measurement setup}

The setup shown in Figure 3.13 allowed to age the AMR sensors, while the setup presented in Figure 3.14 was used to measure the sensor outputs at several magnetic angles. With the sensor powered at $5 \mathrm{~V}$, its output voltages were measured at room temperature at eight angles. These have been set between 0 and 180 degrees using as reference the generated magnetic field of 400 Gauss and varying the angle by the 
movement of the sensor with the rotation disk in the setup.

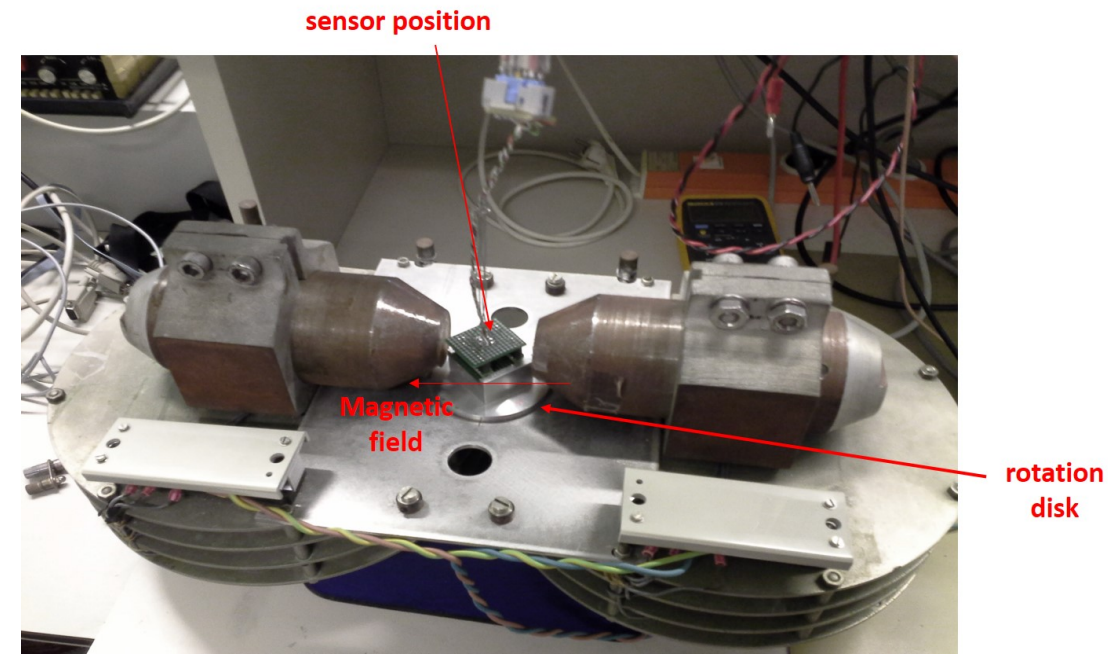

Figure 3.14: Setup used to measure the sensor outputs in the first aging test.

The output voltages of the AMR sensors were measured with two commercial digital multimeters (DMM) connected to a virtual instrument implemented in LabVIEW, as shown in Figure 3.15. The Virtual instrument (VI) which is available in Appendix A refers to a program developed in LabVIEW to acquire the measurements executed by the multimeters and to save the results into a file for further processing. The offset voltages and the signal amplitudes were derived using MATLAB to obtain the Lissajous curves from the sensor outputs.

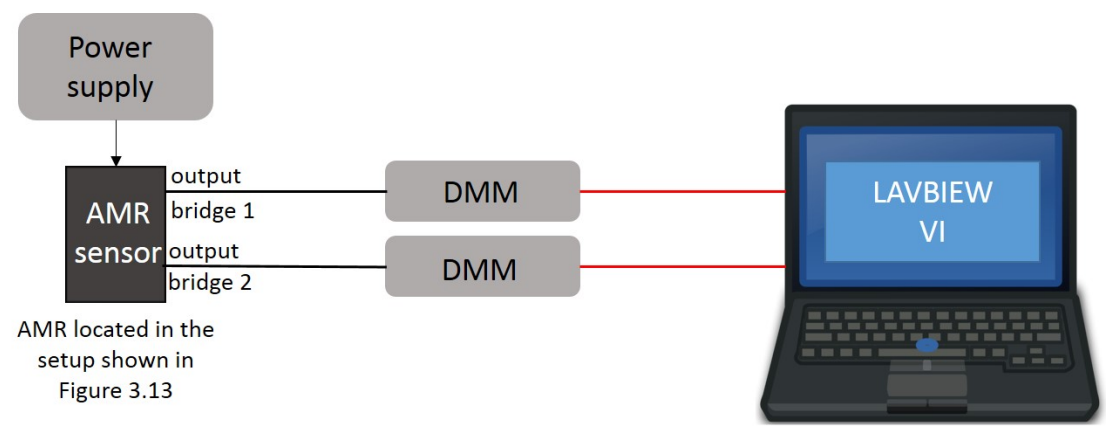

Figure 3.15: Setup of the equipment used to measure the sensor outputs in the first aging test. 


\section{Test Procedure}

Due to time contrains to execute the aging test, it was decided to perform 165 temperature cycles in which the sensors were subjected to the stress conditions presented in section 3.3.1. The sensor outputs were measured at room temperature before and after the temperature cycling. To verify the repeatability of the measurements using the setup presented in section 3.3.1, the measurements were repeated three times to calculate the standard deviation, which resulted in the order of $10^{-5} \mathrm{~V}$ for measurements around $10^{-3} \mathrm{~V}$, indicating that precise measurements were obtained [Lab14].

\section{Results}

The test was focussed on studying the aging effects on the offset voltage and the amplitude of the signals at the sensor outputs. All sensors showed variations in both parameters while comparing the values at the start and the end of the test. Figures 3.16 and 3.17 show the offset voltages at the beginning and the end of the test in bridge 1 and 2 respectively. While in bridge 1 sensor 2 shows the most significant change with values from $-325 \mu \mathrm{V} / \mathrm{V}$ to $-81 \mu \mathrm{V} / \mathrm{V}$, in bridge 2 , it is sensor 3 with values from $-647 \mu \mathrm{V} / \mathrm{V}$ to $-347 \mu \mathrm{V} / \mathrm{V}$. However, all the offset voltages remained well within the range specified by the datasheet $( \pm 2000 \mu \mathrm{V} / \mathrm{V})$.

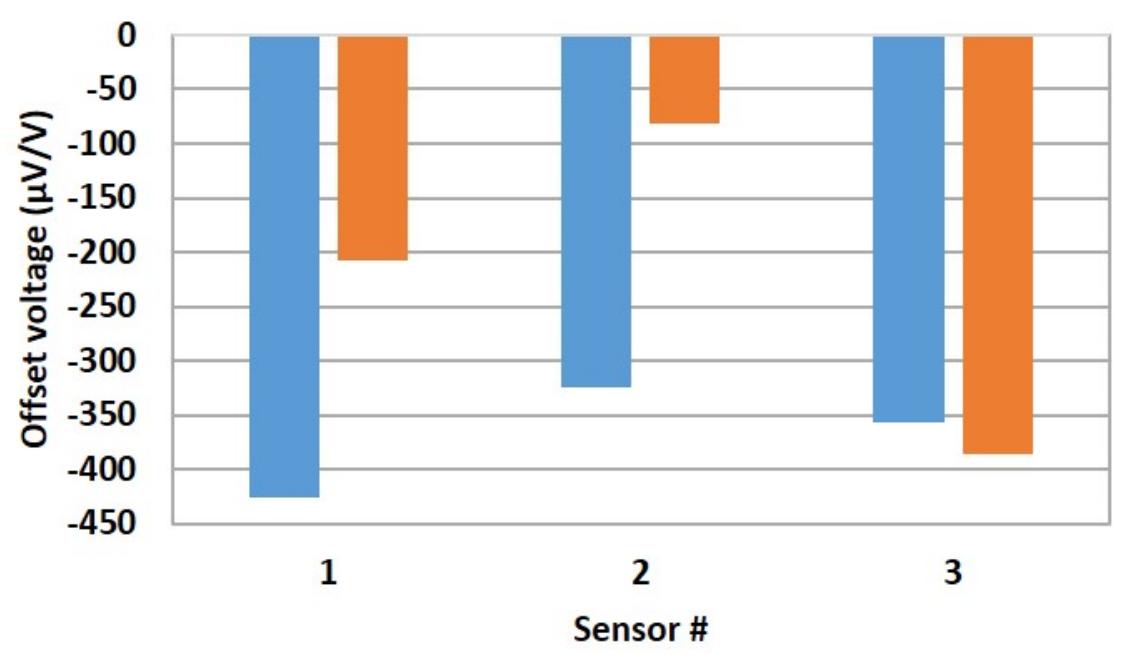

Before Temp. Cycling $\quad$ After Temp. Cycling

Figure 3.16: Offset voltages in bridge 1 of the AMR sensors submitted to ADT. Sensor 2 shows the largest variation with $244 \mu \mathrm{V} / \mathrm{V}$. 


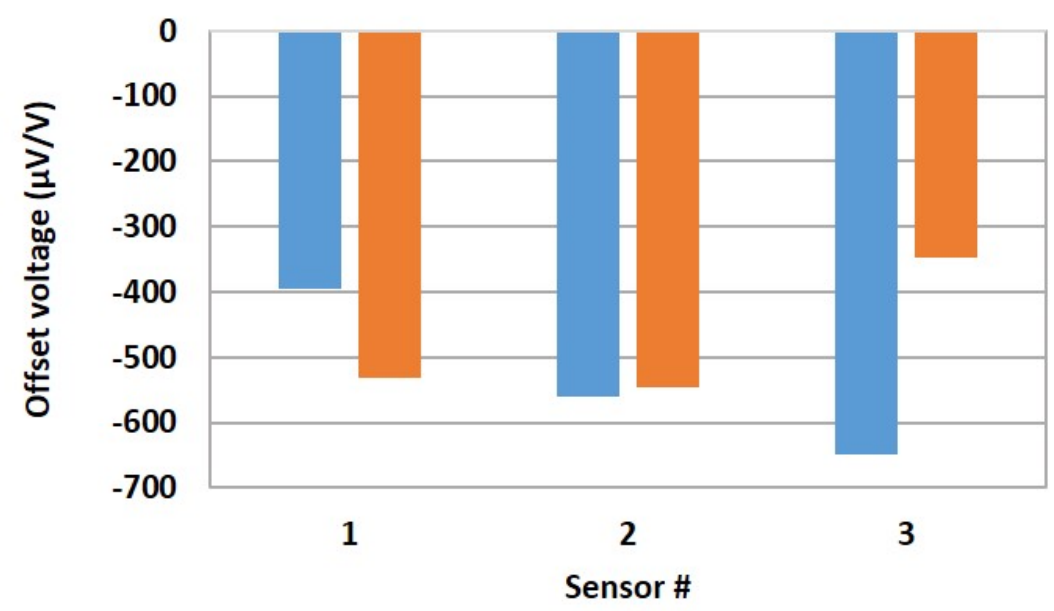

Before Temp. Cycling after Temp. Cycling

Figure 3.17: Offset voltages in bridge 2 of the AMR sensors submitted to ADT. Sensor 3 shows the largest variation with $300 \mu \mathrm{V} / \mathrm{V}$.

Concerning the amplitude of the signals resulting from the magnetoresistance effect (AMR value), the following remarks can be made: Figures 3.18 and 3.19 show the amplitudes in bridge 1 and 2 respectively. Sensor 1 shows the largest variation in bridge one from $68.88 \mathrm{mV}$ to $70.16 \mathrm{mV}$, while in bridge two it is sensor 3 with values from $69.49 \mathrm{mV}$ to $70.35 \mathrm{mV}$. Nevertheless, all the values are within the range indicated by the datasheet (Table 3.1).

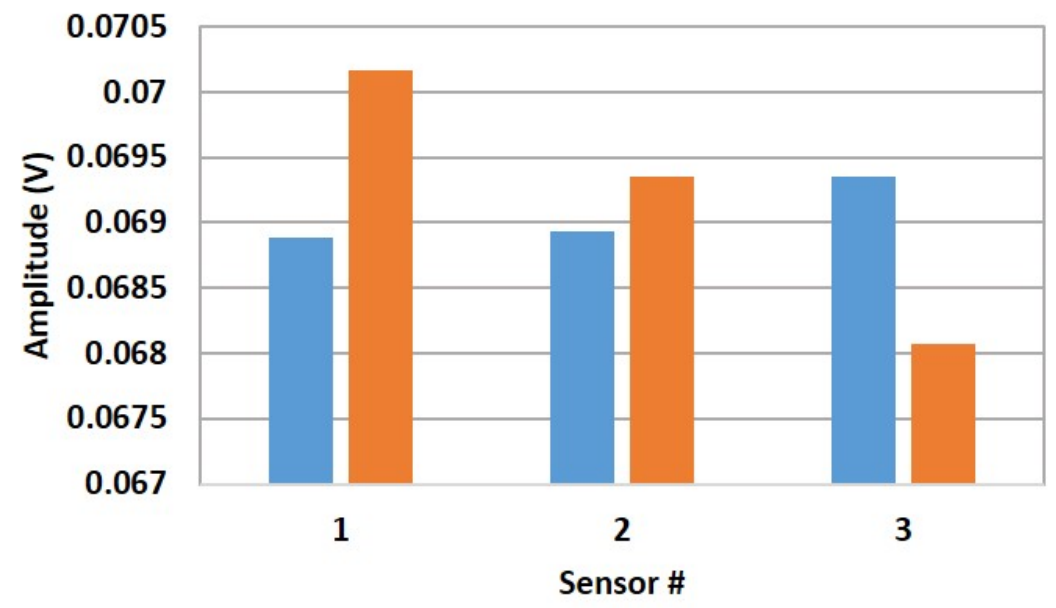

Before Temp. Cycling $\quad$ After Temp. Cycling

Figure 3.18: Amplitude of the signals in bridge 1 of the sensors subjected to ADT. Sensor 1 shows the largest variation with $1.28 \mathrm{mV}$. 


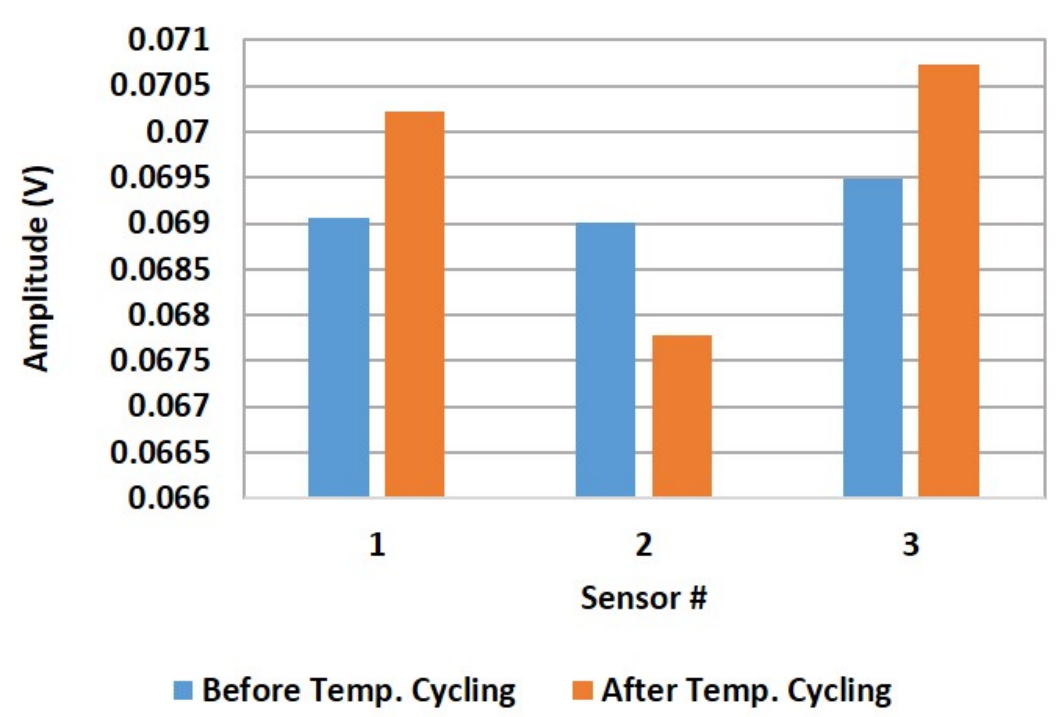

Figure 3.19: Amplitude of the signals in bridge 2 of the sensors subjected to ADT. Sensor 3 shows the largest variation with $0.86 \mathrm{mV}$.

The variations shown by the offset voltages and the amplitudes of the sinusoidal signals at the sensor outputs are most probably linked to changes in the properties of permalloy material over time. This is due to the thermal stress generated from the temperature cycling which represents a stress factor similar to the sensor's operating conditions in automotive applications. The test allowed to verify that the sensor performance drifts over time. However, due to limitations of the measurement setup, only three sensors were subjected to aging and their outputs were measured at the beginning and the end of the test. Therefore it was decided to perform a second batch of tests in which the stress times were longer, with a larger number of sensors and several read points during the test.

\subsubsection{The second aging test}

The second aging test was aimed to continue a further investigation on the aging effects on the sensor parameters of offset voltage and signal amplitudes, but now also the bridge resistances and the angle error were included. Twenty AMR sensors (KMZ49) were subjected to aging during 1709 hours under various stress conditions. Four sensors were not aged and used as reference to verify that the variations obtained in the aged sensors are due to the aging test. In the following, the test is presented in detail, including the setup used to age the sensors, setup used to perform the required measurements at several read points, test procedure and test results.

\section{The aging experiment setup}

During testing the sensors were divided into five test groups, as listed in Table 3.3. 
Table 3.3: Stress conditions during the second aging test

\begin{tabular}{|c|c|c|c|c|c|c|c|}
\hline Phase & Time(hours) & stress conditions & Group 1 & Group 2 & Group 3 & Group 4 & Group 5 \\
\hline \multirow[t]{4}{*}{1} & \multirow[t]{4}{*}{$0-508$} & Power supply voltage & $5 \mathrm{~V}$ & $8 \mathrm{~V}$ & $5 \mathrm{~V}$ & $8 \mathrm{~V}$ & $0 \mathrm{~V}$ \\
\hline & & Temperature & $170^{\circ} \mathrm{C}$ & $170^{\circ} \mathrm{C}$ & $170^{\circ} \mathrm{C}$ & $170^{\circ} \mathrm{C}$ & room temp \\
\hline & & Number of sensors & 4 & 4 & 6 & 6 & 4 \\
\hline & & Magnet & yes & yes & no & no & no \\
\hline \multirow[t]{5}{*}{2} & \multirow[t]{5}{*}{$508-1283$} & Power supply voltage & $8 \mathrm{~V}$ & $8 \mathrm{~V}$ & $8 \mathrm{~V}$ & $8 \mathrm{~V}$ & $0 \mathrm{~V}$ \\
\hline & & Temperature & $(50-187)^{\circ} \mathrm{C}$ & $(50-187)^{\circ} \mathrm{C}$ & $(50-187)^{\circ} \mathrm{C}$ & $(50-187)^{\circ} \mathrm{C}$ & room temp \\
\hline & & Temp cycles & 545 & 545 & 545 & 545 & 0 \\
\hline & & Number of sensors & 4 & 4 & 6 & 6 & 4 \\
\hline & & Magnet & yes & yes & no & no & no \\
\hline \multirow[t]{5}{*}{3} & \multirow[t]{5}{*}{$1283-1709$} & Power supply voltage & $8 \mathrm{~V}$ & $8 \mathrm{~V}$ & $8 \mathrm{~V}$ & $8 \mathrm{~V}$ & $0 \mathrm{~V}$ \\
\hline & & Temperature & $(50-207)^{\circ} \mathrm{C}$ & $(50-207)^{\circ} \mathrm{C}$ & $(50-207)^{\circ} \mathrm{C}$ & $(50-207)^{\circ} \mathrm{C}$ & room temp \\
\hline & & Temp cycles & 134 & 134 & 134 & 134 & 0 \\
\hline & & Number of sensors & 4 & 4 & 6 & 6 & 4 \\
\hline & & Magnet & yes & yes & no & no & no \\
\hline
\end{tabular}

The duration of each phase, as well as the maximum temperature, were defined along the test based on the results obtained. 
The first four groups were submitted to aging via the execution of an ADT, in which constant and step stress (CSADT and SSADT) have been combined to define the stress levels. Constant stress refers to the usage of several groups exposed to different conditions, while step stress means the variation of the stress conditions of each group during the test. Group 5 includes the reference sensors not submitted to aging, which is aimed to verify that the variations of the parameters in the sensors of the first groups are indeed due to the aging test.

The various stress conditions include magnetic field, power supply, and temperature. The magnetic field was applied in two of the four aged groups. Regarding the power supply, $5 \mathrm{~V}$ which is the voltage used in normal operation and $8 \mathrm{~V}$ as the higher stress level have been applied. For the temperature, temperature cycling has been applied based on the temperature profile experienced by automotive electronics presented in chapter 2 , but also a high temperature because it is commonly used in aging tests and it allows to simulate periods in which the car is used continuously for a long time [AEC13], [ZVE13].

Figure 3.20 shows the schematic of the setup used to age the AMR sensors. It includes a temperature chamber used to apply the temperature stress, power supplies to power the sensors and a digital multimeter connected to a switching system. These last two instruments were connected to a VI (Virtual Instrument), developed in LabVIEW (Appendix A), to monitor the voltages supplied to the sensors, as well as the temperature on the test board.

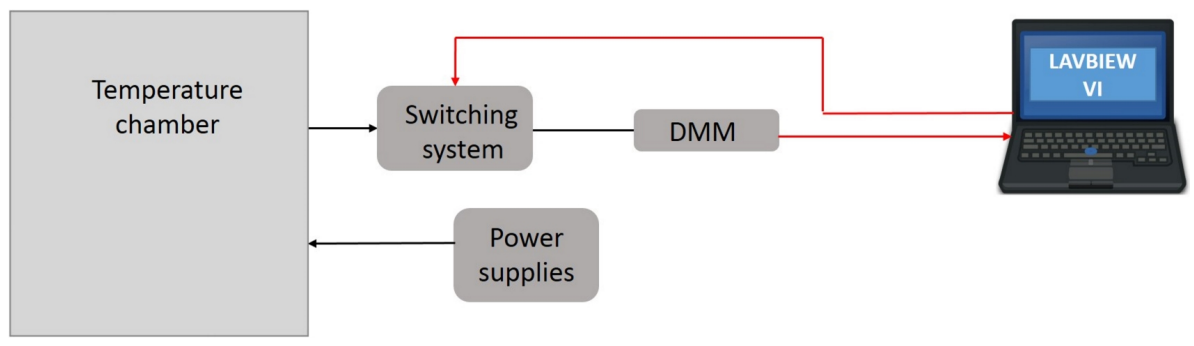

Figure 3.20: Schematic of the setup used to age the AMR sensors during the second aging test.

Figure 3.21 shows the test board used to place the sensors in the temperature chamber. This board was designed for this experiment and made with the material Rogers 4350 that is able to withstand high temperatures. The sockets are nonmagnetic sockets manufactured by the Loranger corporation which are designed for magnetic tests. The first eight sockets from left to right have a permanent magnet on top of them. The board includes five temperature sensors, one in each corner and one in the centre, which have been used to monitor the temperature during the test. The connectors at the left side of the board were also used to apply the required voltages to the sensors. 


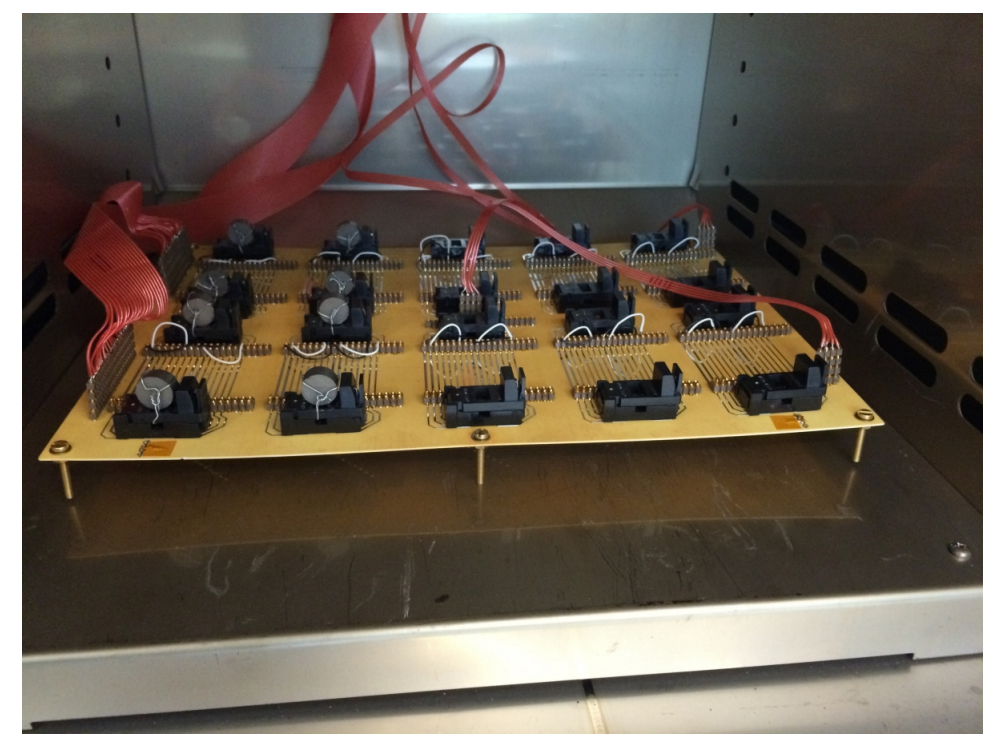

Figure 3.21: Test board used to place the sensors in the temperature chamber during the second aging test. The sensors in the first two columns from left to right have magnets on top.

\section{Measurement setup}

During the aging test, 12 reading points listed in Table 3.4 that is shown in section 3.3.2 have been used, in which the sensors were removed from the test board presented in Figure 3.21. For each read point, a set of 17 measurements were performed at room temperature in each sensor in 20 equidistant angles between 0 and 180 degrees. Figure 3.22 shows a schematic of the measurement setup in which a source measurement unit (SMU) configured for 4-point measurements is included, as well as a digital multimeter (DMM) and a switching system. This last hardware allows making the required configurations to take all the measurements at each angle. A VI developed in LabVIEW, which is presented in Appendix A, has been used to acquire the measurements from the SMU and DMM, but also to indicate the configurations should be defined with regard to the switching system, meaning the status open/close of the switches.

The 17 measurements are listed in Figure 3.23. Four of them measure the differential outputs of the sensor using the power supply connected in normal configuration and with power supply reversed. This means that in the normal configuration the $(+)$ and (-) of the power supply are connected to the power supply (VCC) and ground (GND) of the sensor, but in power-supply reversed (+) and (-) are connected to GND and VCC. The aim was to suppress the offset of the measurement instruments and thermovoltages to obtain more accurate results. Twelve measurements were carried out to determine the bridge resistances and one measurement to monitor the temperature. 


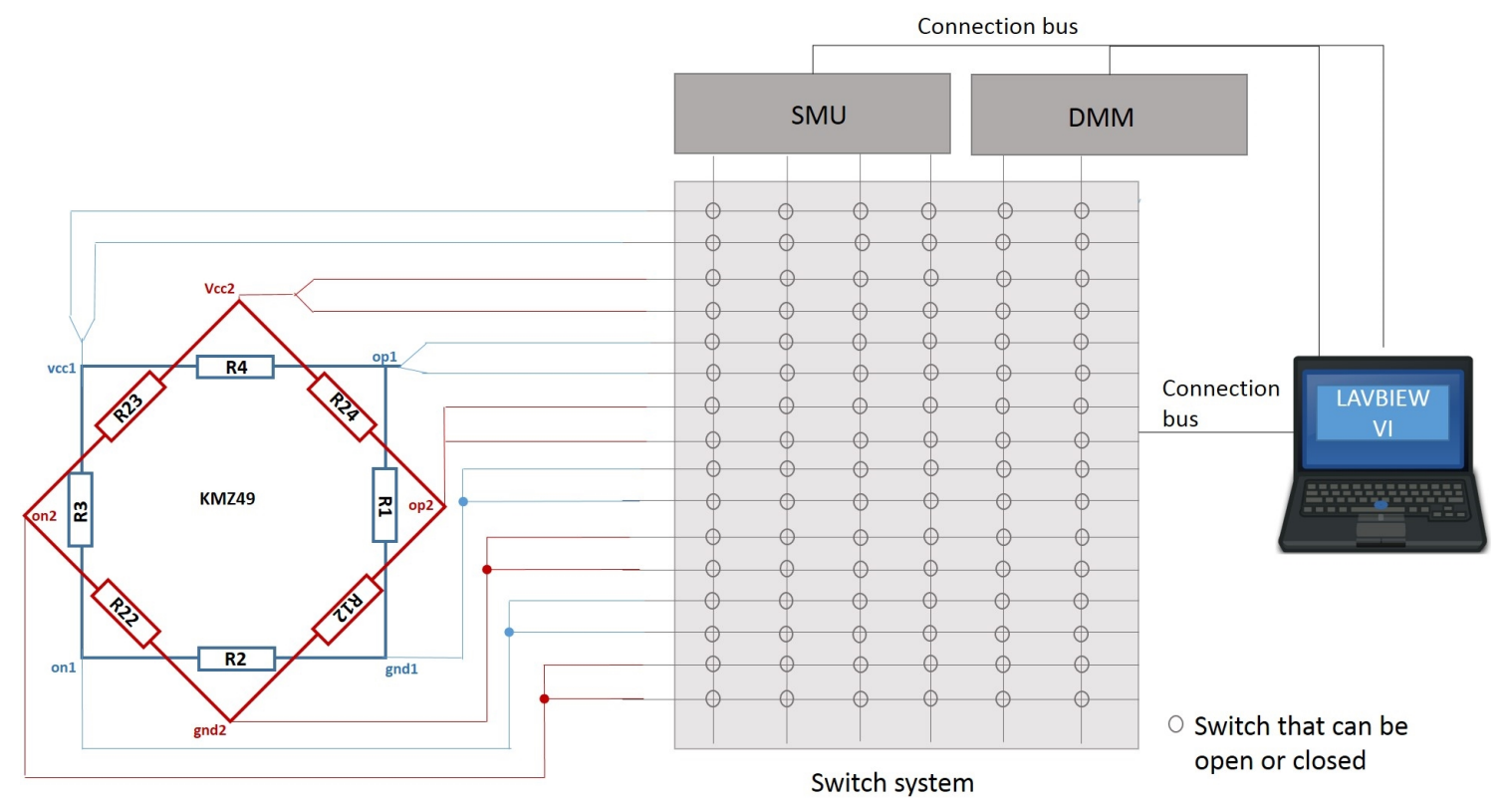

Figure 3.22: Setup of the equipments used to perform the required measurements in the second aging test. 


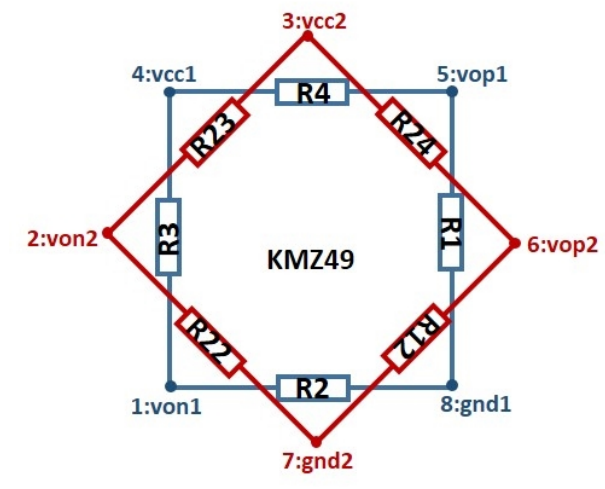

\begin{tabular}{|c|c|cc|}
\hline Measurements & DMM & \multicolumn{2}{|c|}{ SMU } \\
\hline \multirow{2}{*}{ output voltage of bridge 1 } & V51 & V48 & 148 \\
& V51 & V84 & 184 \\
\hline \multirow{2}{*}{ output voltage of bridge 2 } & V62 & V37 & 137 \\
& V62 & V73 & 173 \\
\hline & V41 & V48 & 148 \\
& V81 & V48 & 148 \\
Measurements to calculate & V45 & V48 & 148 \\
resistances of bridge 1 & V85 & V48 & 148 \\
& V41 & V51 & 151 \\
& V45 & V51 & 151 \\
\hline \multirow{3}{*}{ Measurements to calculate } & V32 & V37 & 137 \\
resistances of bridge 2 & V72 & V37 & 137 \\
& V76 & V37 & 137 \\
& V32 & V37 & 137 \\
Temperature & V36 & V62 & 162 \\
\hline
\end{tabular}

The numbers correspond to the pins in the KMZ49

The temperature sensor is shown in Figure 3.25

Figure 3.23: List of the measurements made on the AMR sensor KMZ49. 
Figure 3.24 shows the setup used to set the angle between the magnetic field and the sensor. The angle was changed by the movement of a permanent magnet of 400 Gauss located at the bottom part, which is connected to the rotating disk used to define the angle. Figure 3.25 depicts a top view of the setup in which the test board with the Loranger socket for the AMR sensor and a temperature sensor under the AMR sensor can be observed, as well as the connections to the switch system (see Figure 3.22). A power supply of $5 \mathrm{~V}$ was used to power the sensors.

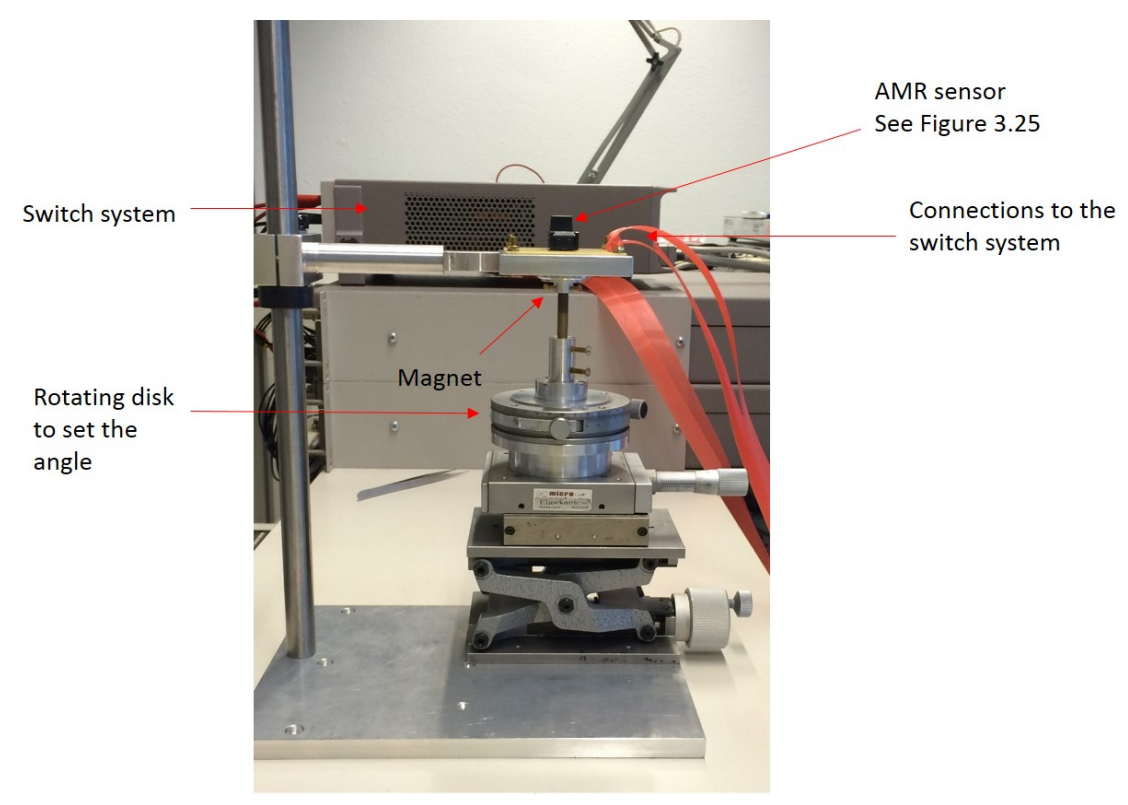

Figure 3.24: Setup used to measure the sensor outputs in the second aging test.

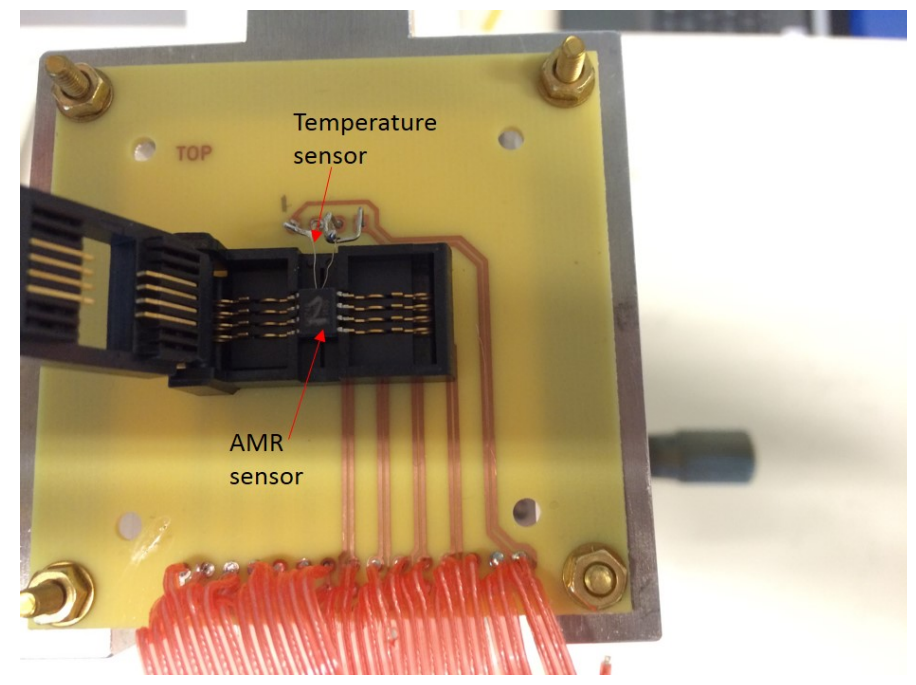

Figure 3.25: Top view of the setup used to set the angles and measure the sensor outputs in the second aging test. 


\section{The test procedure}

The aging test can be divided into three phases based on the temperature stress conditions. In these phases, the test groups shown in Figure 3.26 were subjected to various stress conditions as presented in Table 3.3 and it is detailed below. The duration of each phase, as well as the maximum temperature for temperature cycling in phase 2 and 3 were defined based on the results we were obtaining throughout the test.

- Phase 1: during the first 508 hours, a high temperature of $170^{\circ} \mathrm{C}$ was set to stress the sensors. This value represents a higher level of the maximum temperature $\left(150{ }^{\circ} \mathrm{C}\right)$ indicated in the datasheet of the KMZ49 [NXP11]. The test groups 1 and 3 were powered with $5 \mathrm{~V}$, while groups 2 and 4 were powered with $8 \mathrm{~V}$. Regarding the magnetic field, sensors of groups 1 and 2 had a magnet on top of 752 Gauss, which is sufficiently strong to take the sensor into its saturation state.

- Phase 2: this started after phase one and lasted for 775 hours. During this time 545 temperature cycles were performed in a range between $\left(50^{\circ} \mathrm{C}-187^{\circ} \mathrm{C}\right)$, defined as a higher level from the ambient and maximum temperature $\left(25^{\circ} \mathrm{C}\right.$, $150^{\circ} \mathrm{C}$ ) indicated in the datasheet of the KMZ49 [NXP11]. The magnetic stress remained like in the previous phase, but all the sensors were powered with an $8 \mathrm{~V}$ stress.

- Phase 3: this phase lasted for 426 hours in which 134 cycles were executed in a temperature range between $\left(50^{\circ} \mathrm{C}-207^{\circ} \mathrm{C}\right)$. In terms of power supply and magnetic field, these factors remained the same as in phase two.

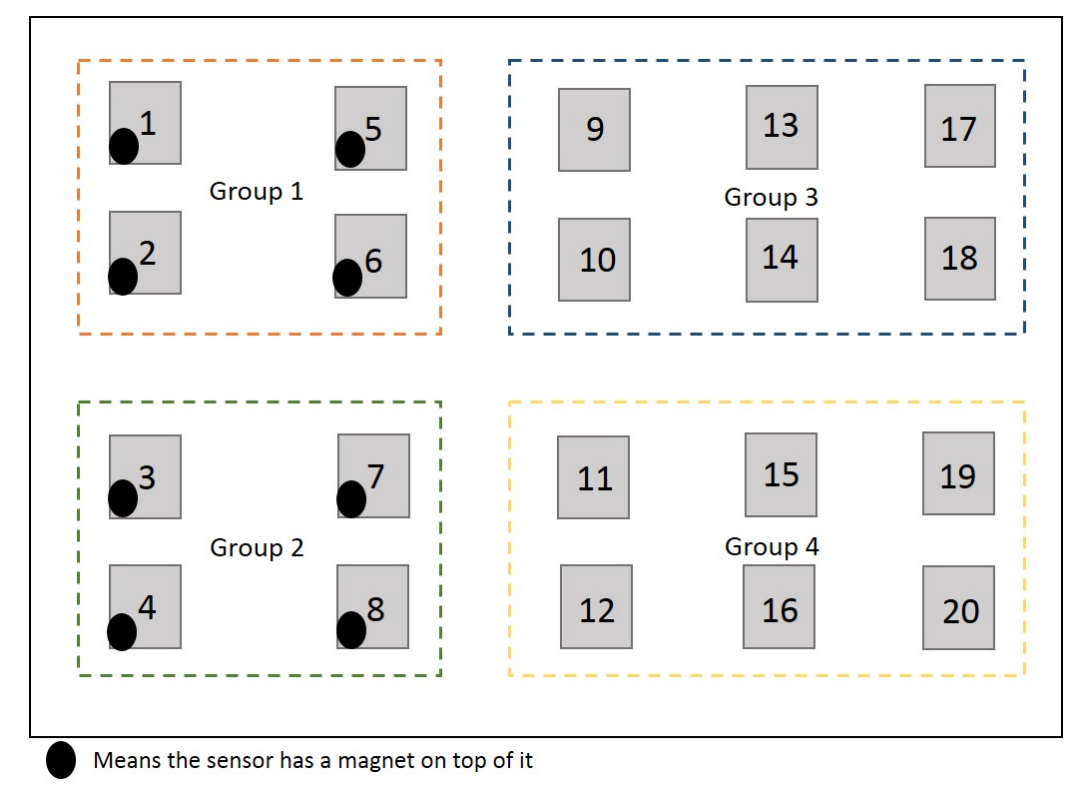

Figure 3.26: Distribution of the test groups on the board used to age the sensors in the temperature chamber during the second aging test. The numbers correspond to the numbers assigned to each sensor. 
During the test time, 12 read points have been defined as shown in Table 3.4 in which the read points as well as their time and phase of the test are included. For each read point, the measurements indicated in section 3.3.2 have been performed in all the sensors including the sensors of group 5 and maintaining an average temperature of $30^{\circ} \mathrm{C}\left( \pm 1.7^{\circ} \mathrm{C}\right)$ on the sensors. The repeatability of the measurements was verified throughout the aging test by repeating three times the measurements of 3 sensors at each read point. The standard deviation was always in the order of $10^{-5} \mathrm{~V}$ for measurements in the range of $10^{-3} \mathrm{~V}$, which indicates that precise measurements were obtained.

The sensor parameters presented in the next section were determined as described in section 3.2. The calculations were carried out in MATLAB using the voltages measured at the sensors' outputs. The sensor parameters $\left(\right.$ sensor $\left._{\text {par }}\right)$, such as offset voltage, bridge resistances and amplitude, have been temperature compensated $\left(\right.$ sensor $_{\text {parcom }}$ ) with respect to the temperature of the measurements at 0 hours $\left(\right.$ Temp $\left.p_{\text {ohours }}\right)$ and taking into consideration the temperature at the read point (Temp $\left.p_{\text {readpoint }}\right)$, as indicated in Equation (3.13). This was done to reduce the temperature effects over the drift trends of the sensor parameters.

$$
\text { sensor }_{\text {parcom }}=\text { sensor }_{\text {par }}(\text { readpoint })-T C *\left(\text { Temp }_{\text {readpoint }}-\text { Temp }_{0 \text { hours }}\right)
$$

Table 3.4: Read points during the second aging test

\begin{tabular}{|c|c|c|}
\hline Read point & Test time (hours) & Test phase \\
\hline 1 & 0 & 1 \\
2 & 1 & 1 \\
3 & 10 & 1 \\
4 & 28 & 1 \\
5 & 163 & 1 \\
6 & 298 & 1 \\
7 & 508 & $1-2^{[1]}$ \\
8 & 646 & 2 \\
9 & 831 & 2 \\
10 & 977 & 2 \\
11 & 1283 & $2-3{ }^{[1]}$ \\
12 & 1709 & 3 \\
\hline
\end{tabular}

[1] transition between phases 
The temperature coefficients $(T C)$ that have been used are, for offset voltage $2.10^{-6}$; for resistance 8.42 and for amplitude -0.0003 , which have been calculated following the procedure explained next for the amplitude. In Figure 3.27 is shown the plot of temperature versus amplitude for a bridge in one of the AMR sensors KMZ49, in which the temperature coefficient is equal to the slope of the line. This procedure was applied to all sensor bridges to subsequently calculate the average value of $T C$ which was compared with the range specified in the datasheet of the KMZ49 (Table 3.1) for verification purposes.

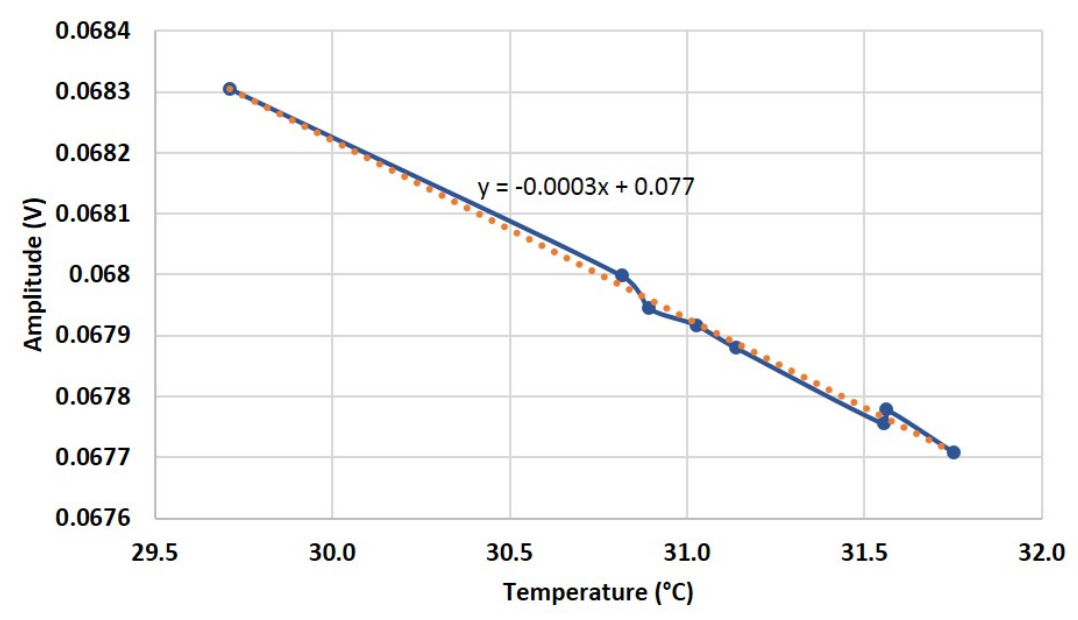

Figure 3.27: Temperature versus amplitude in one bridge of an AMR sensor KMZ49.

\section{The experimental results}

This second aging test has been performed to continue a further investigation on the aging effects on the performance of AMR sensors used for angle measurements. In the following, the results obtained concerning the offset voltage, signal amplitudes at the sensor outputs, amplitude imbalance between them, bridge resistances, and angle error are presented. Because in this research we are more interested in the average trend of variation among the sensors, most of the results are presented based on the average value of each test group. The average represents the arithmetic mean and it has been calculated by adding the values of each sensor in the group and then dividing them by the number of sensors in the group, as indicated in Equation (3.14). These results will be analysed in the next section.

$$
\text { average }=\frac{S_{1}+S_{2}+\ldots S_{n}}{n}
$$

where $S$ represents the value of each sensor and $n$ the number of sensors. 
With regard to the Offset Voltage, this parameter has been calculated as detailed in section 3.2.2 and subsequently temperature-compensated based on Equation (3.13). The values show variations along the test in all the aged sensors as shown in Figure 3.28. This presents the histogram of the distribution of the sensor bridges, versus the drift of the offset voltage at the end of the test in a range between $0 \mu \mathrm{V} / \mathrm{V}$ and $150 \mu \mathrm{V} / \mathrm{V}$ divided into 6 bins. Half of the bridges show a drift larger than $50 \mu \mathrm{V} / \mathrm{V}$.

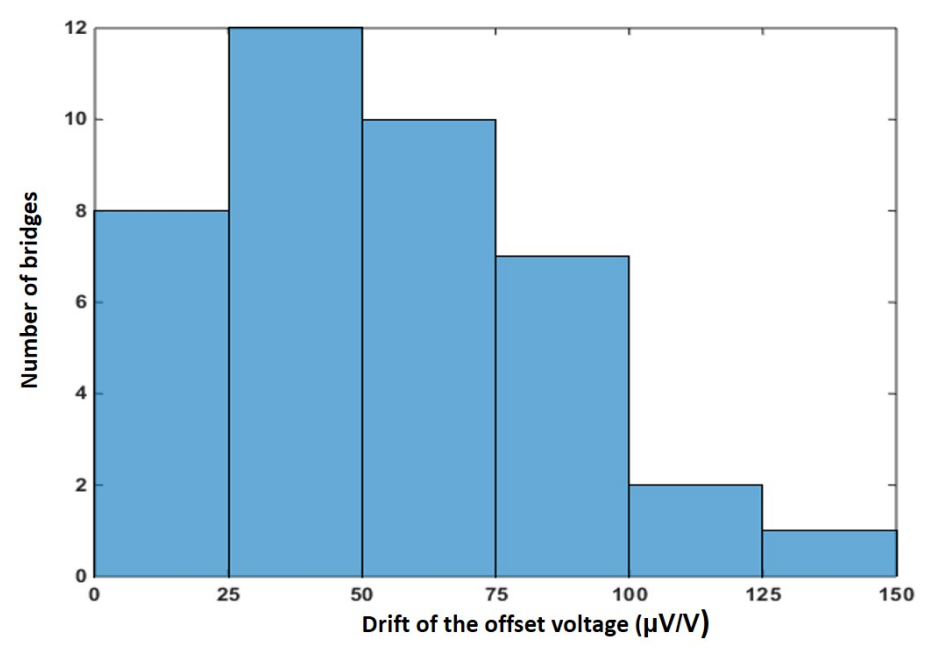

Figure 3.28: Histogram of the drift of the offset voltages in the 40 bridges of the 20 aged sensors at the end of the second aging test. The drift ranges between zero and $150 \mu \mathrm{V} / \mathrm{V}$ and is divided into 6 bins.

The drift over time of the offset voltage in each bridge of the sensors has been obtained by subtracting the offset voltages at 0 hours from the values obtained at the other read points. Then the average of the drift in each test group was calculated as shown in Equation (3.14), of which the results are shown in Figures 3.29 and 3.30 for bridge 1 and 2 respectively. In general, a trend better shown for bridge 1 than for bridge 2 indicates that the drift of the offset voltage presents large variations at the start but slow down over time. If one compares the values at the end of the test, group 2 shows the largest drift in bridge one with $77 \mu \mathrm{V} / \mathrm{V}$ and group 4 in bridge two with $73 \mu \mathrm{V} / \mathrm{V}$. By comparing the results of the aged groups with group 5 it is evident that the drifts of the aged groups are due to the aging. The variations of the trend between 400 - 600 hours and after 1300 hours could be a response to the changes in the temperature stress conditions between the phases of the test. 


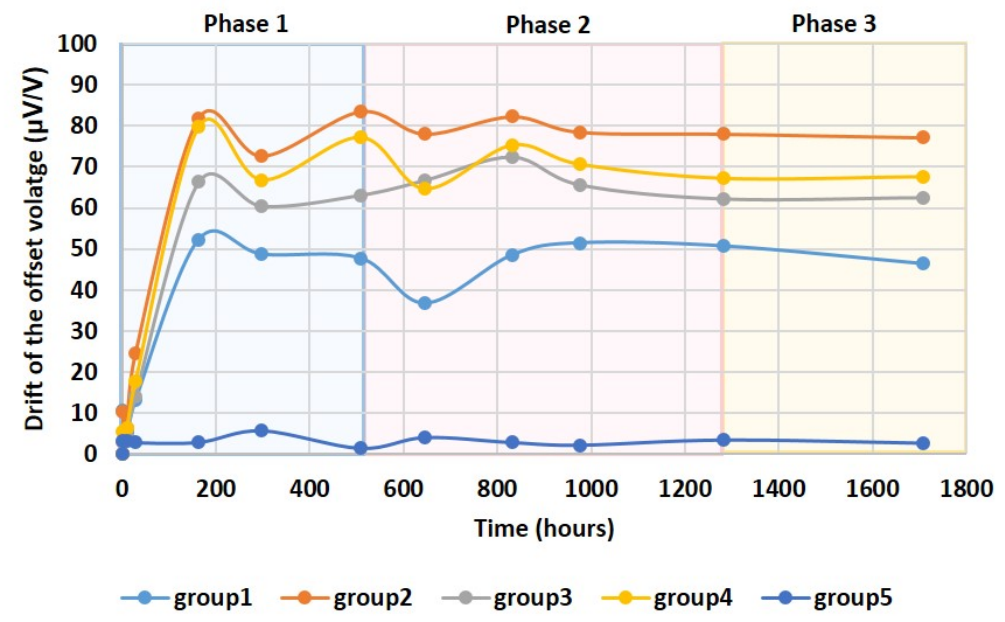

Figure 3.29: Average of the drift of the offset voltage of bridge 1 in each test group.

Phase 1, 2 and 3 indicate the phases of the test as detailed in Table 3.3.

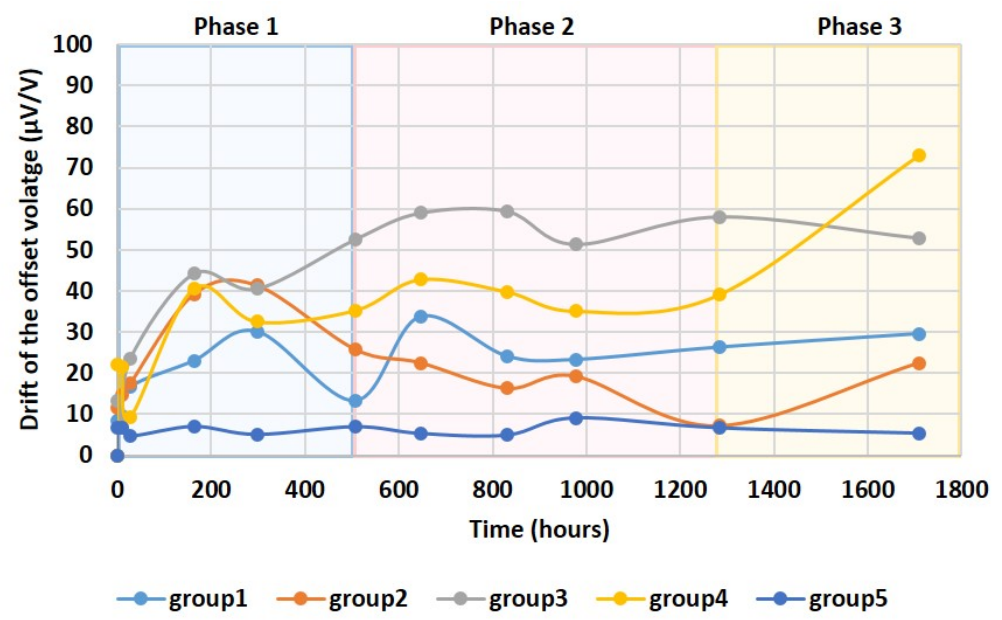

Figure 3.30: Average of the drift of the offset voltage of bridge 2 in each test group. Phase 1, 2 and 3 indicate the phases of the test as detailed in Table 3.3.

The effects of the stress conditions of the magnetic field and the power supply for the sensors are not completely clear. In bridge one, as mentioned before, group 2 with a magnet on top shows the largest drift, but group 1 that also has a magnet shows the smallest drift. This also occurs in these two groups in bridge 2 . With regard to the two voltages used to power the sensors in phase 1 of the test, in bridge 1 , groups 2 and 4 powered with $8 \mathrm{~V}$ show a larger drift as compared to groups 1 and 3 powered with $5 \mathrm{~V}$. However, in bridge 2 group 3 appears the largest drift in the same phase of the test.

Now the amplitude of the sinusoidal signals at the sensor outputs will be discussed. The values of each sensor have been calculated as presented in section 3.2.3 and 
temperature compensated based on Equation (3.13). Then, the averages of the test groups shown in Figures 3.31 and 3.32 for bridge 1 and 2, have been calculated as provided by Equation (3.14). All the values are within the range between $60 \mathrm{mV}$ and $75 \mathrm{mV}$ specified by the datasheet, showing a tendency to increase at the beginning of the test, but decreasing with time in the long run. At the end of the test, group 1 shows the greatest reduction in both bridges with $0.14 \%$ and $0.13 \%$ of their initial values. While comparing the aged groups with group 5 (not aged), this turns out to be more stable during the test time with variations of $0.03 \%$ and $0.05 \%$ in bridge one and two respectively, but there is not a big difference between all of them. Although it is expected that the drift of the aged groups continues over time, the amplitude of the sensor outputs did not show large variations during the aging test.

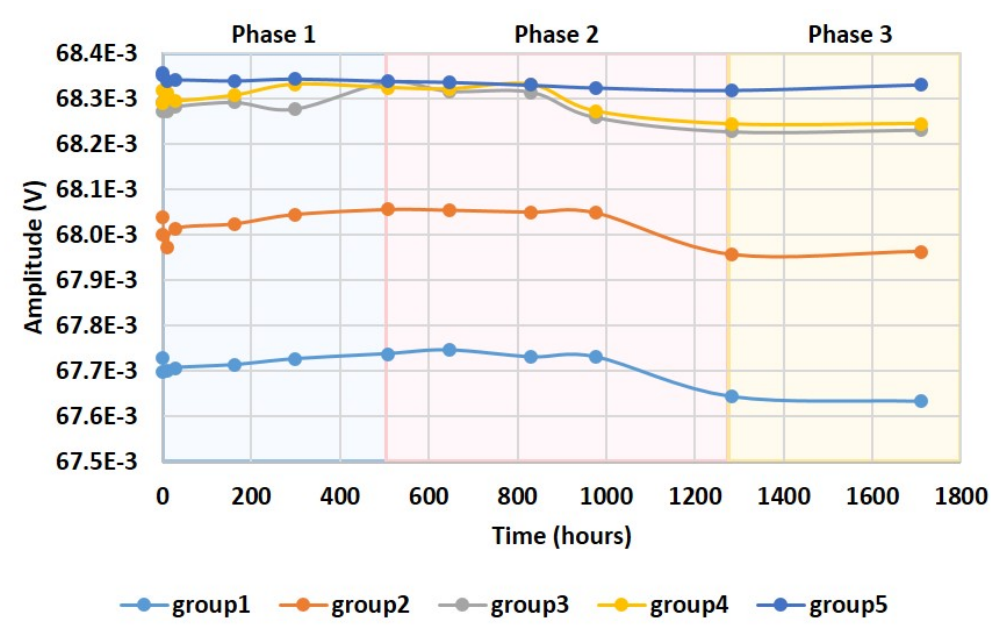

Figure 3.31: Average of the amplitude of the output signals in bridge 1 for each test group. Phase 1, 2 and 3 indicate the phases of the test as detailed in Table 3.3.

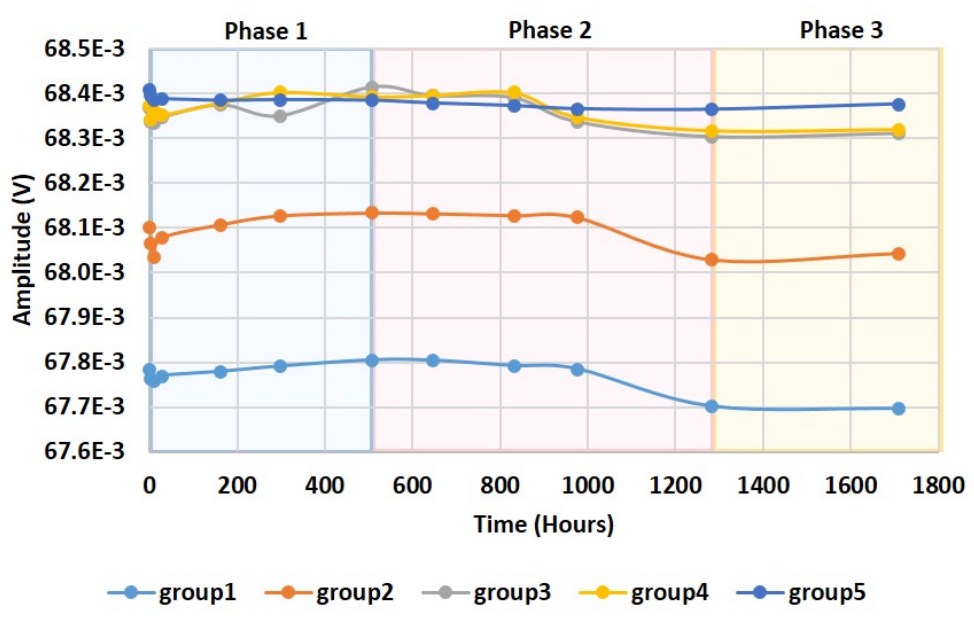

Figure 3.32: Average of the amplitude of the output signals in bridge 2 for each test group. Phase 1, 2 and 3 indicate the phases of the test as detailed in Table 3.3. 
With regard to the amplitude imbalance, this has been measured based on the amplitude synchronism factor $k$ presented in Equation (3.10). Figure 3.33 shows the factor $k$ for the test groups, in which the values move away from $100 \%$ over time for the aged groups. This means that the amplitude imbalance between the bridge outputs increases slowly during the test time being the largest drift in group 4 with $0.03 \%$ at the end of the test. Group 5 (not aged) shows a more stable behaviour over time than the aged groups.

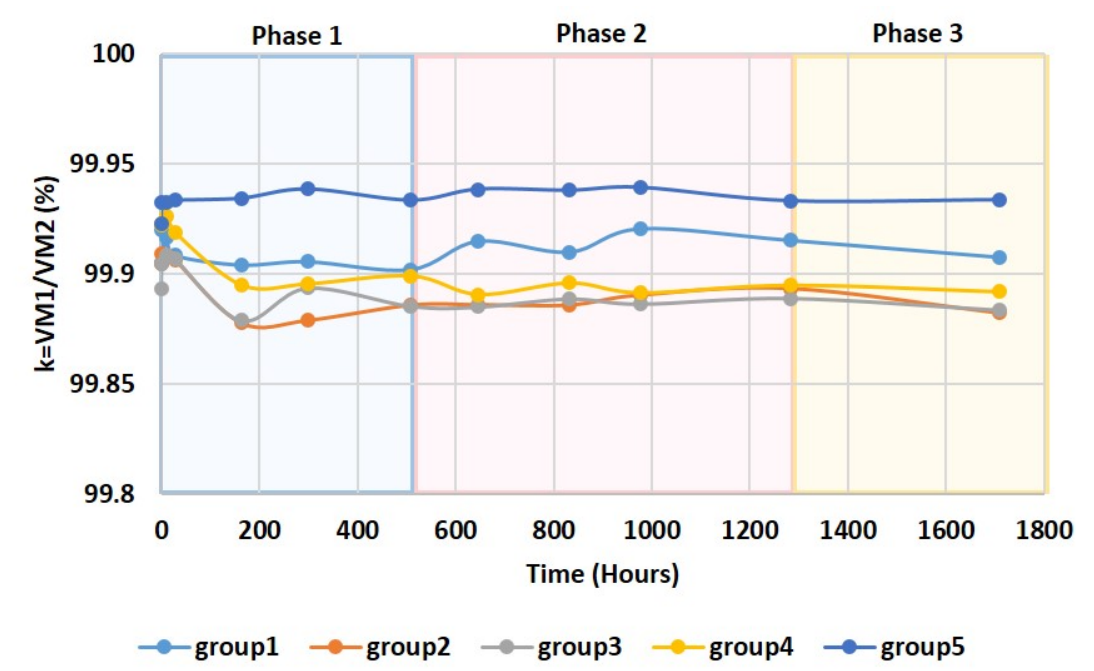

Figure 3.33: Amplitude synchronism factor $k$ in the test groups. Phase 1, 2 and 3 indicate the phases of the test as detailed in Table 3.3.

In the case of the bridge resistances, the average value for each test group has been calculated following the method shown in Figure 3.34. First, the values of the bridge resistances for each sensor were calculated as explained in section 3.2.1. Next, the average value of each resistance has been calculated and then the average value of each bridge. These values were temperature compensated and subsequently the average value per test group was determined.

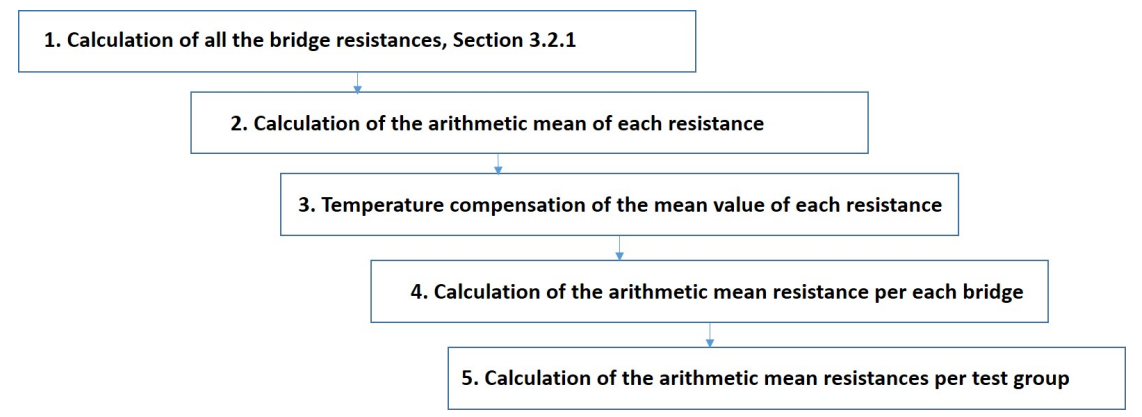

Figure 3.34: Method followed to calculate the average resistance per test group in the second aging test. 
Figures 3.35 and 3.36 show the average resistance of each test group in bridge 1 and 2 respectively in which all the resistance values are within the range of $2.3 \mathrm{k} \Omega$ $3.7 \mathrm{k} \Omega$ specified by the datasheet. The resistances tend to decrease slowly over time in all the aged groups until 1000 hours, showing later a slow tendency to increase. Overall, group 4 shows the largest variation with a reduction of $0.13 \%$ of the initial value in bridge 1 and $0.19 \%$ in bridge 2 . While comparing with group 5 (not aged), this shows a more stable behaviour over time with variations of $0.01 \%$ and $0.04 \%$ in bridge 1 and 2 respectively.

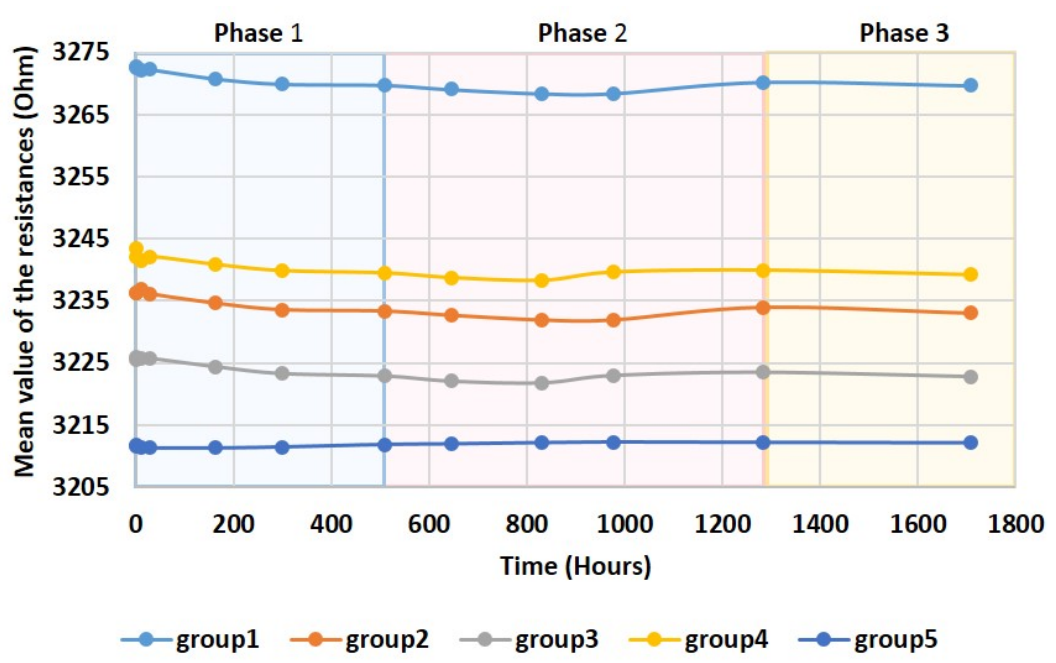

Figure 3.35: Average resistance in bridge 1 for each test group. Phase 1,2 and 3 indicate the phases of the test as detailed in Table 3.3.

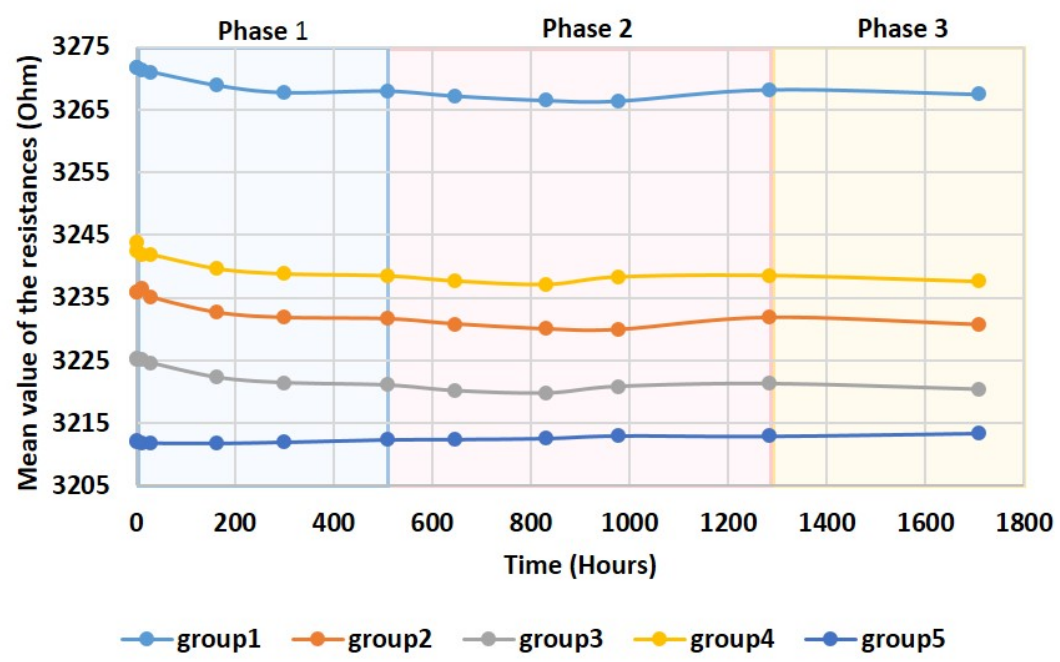

Figure 3.36: Average resistance in bridge 2 for each test group. Phase 1, 2 and 3 indicate the phases of the test as detailed in Table 3.3.

As mentioned before, the undesired parameters included in the sensor outputs are 
because of mismatch between its resistances, hence the mismatch variation during the aging test is also of interest, which should be investigated for each sensor. In general, the results show that the drift trends of the resistances present more variations between them at the start of the test, becoming more similar with time. Figure 3.37 shows the average values of the bridge resistances in sensor S7 as an example, which have been obtained at step three of the procedure depicted in Figure 3.34. In the first phase of the test, the trends of the resistances show more variations between them than in the other two phases of the test. Hence, aging has in the long term a positive effect on the mismatch between the resistances.

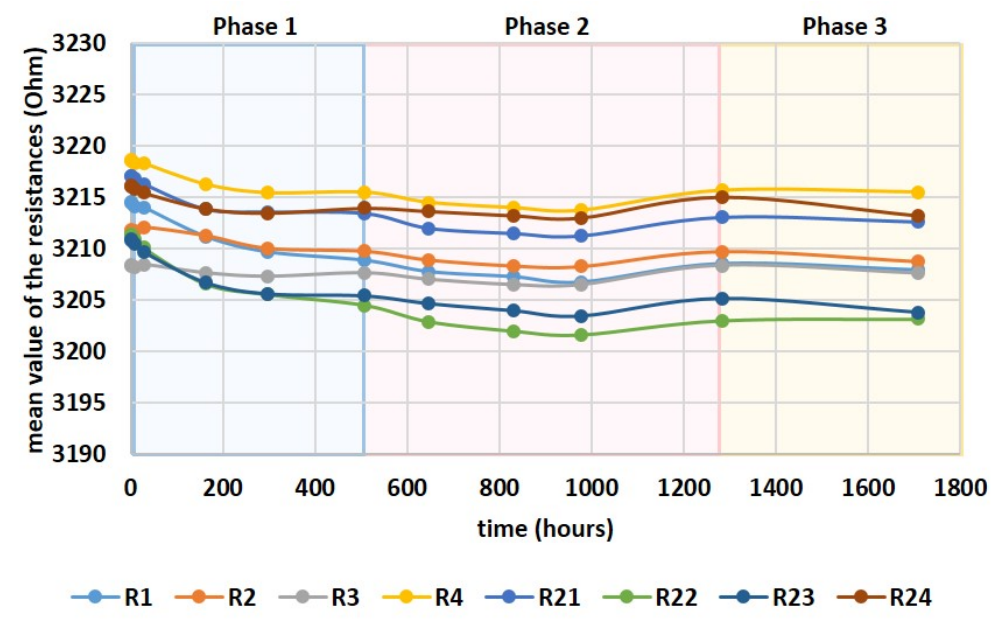

Figure 3.37: Mean value of the bridge resistances in sensor 7. Bridge 1 (R1, R2, R3, R4) and bridge 2 (R21, R22, R23, R24).

Regarding the angle error, our study focussed on the drift over time of the maximum angle errors due to offset voltage, amplitude imbalance and harmonics. The values per test group have been obtained following the methodology as shown in Figure 3.38. First, the maximum angle error due to the three sources in each sensor as explained in section 3.2.4 was calculated. Next, the drift over time was obtained by subtracting the angle errors of each read point from the values obtained at zero hours. Finally, the arithmetic mean of each test group as indicated in Equation (3.14) was calculated.

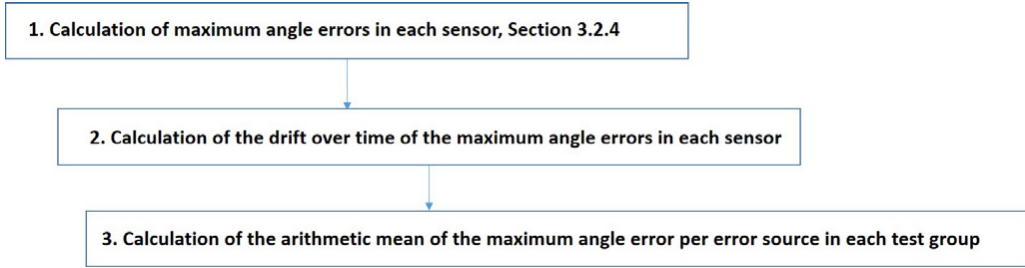

Figure 3.38: Methodology followed to calculate the average of the drifts of the angle errors during the second aging test. 
The results show that the angle errors due to offset voltage have the largest variations over time followed by the angle errors due to amplitude imbalance and finally the harmonics. The trends of the variations are very similar among the aged groups, as shown in Figures 3.39, 3.40, 3.41, and 3.42, which present the drifts of the three sources of angle errors in the test groups 1, 2, 3 and 4 respectively. When the angle errors at the end of the test are compared, group 2 shows the largest drift for offset voltage and harmonics, while group 1 for the amplitude imbalance, as shown in Table 3.5.

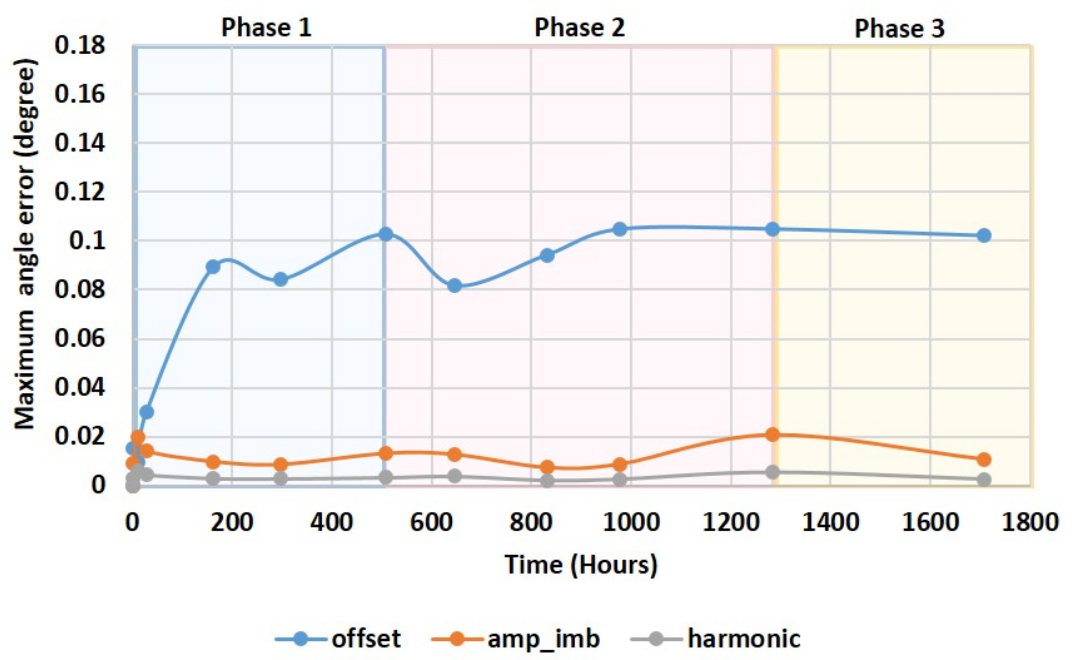

Figure 3.39: Drift of the angle errors in test group 1 during the second aging test.

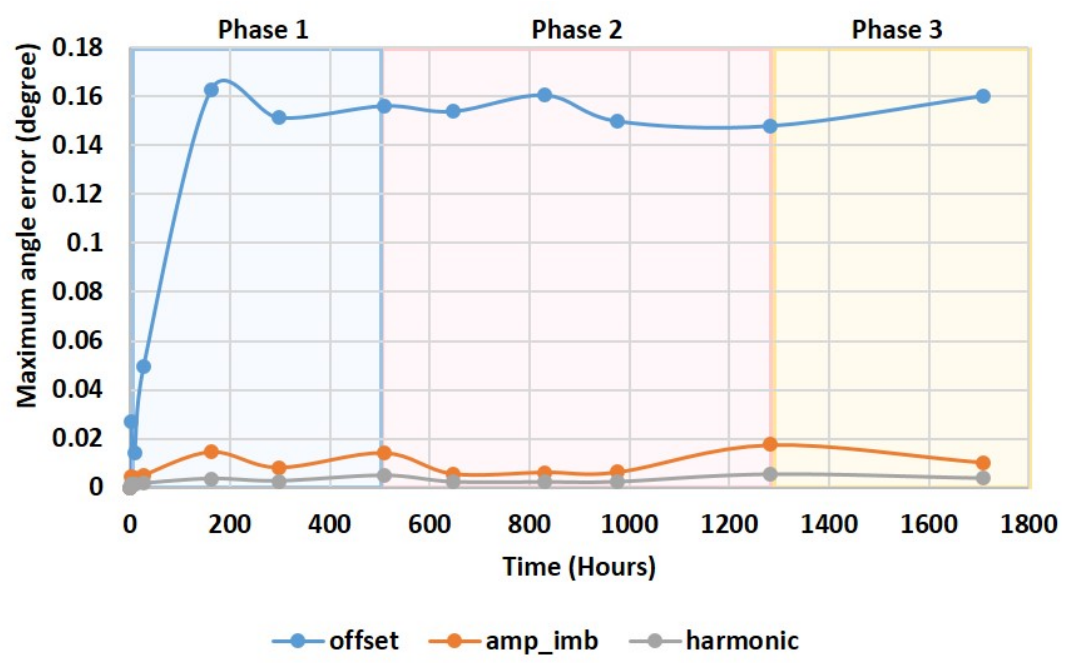

Figure 3.40: Drift of the angle errors in test group 2 during the second aging test. 


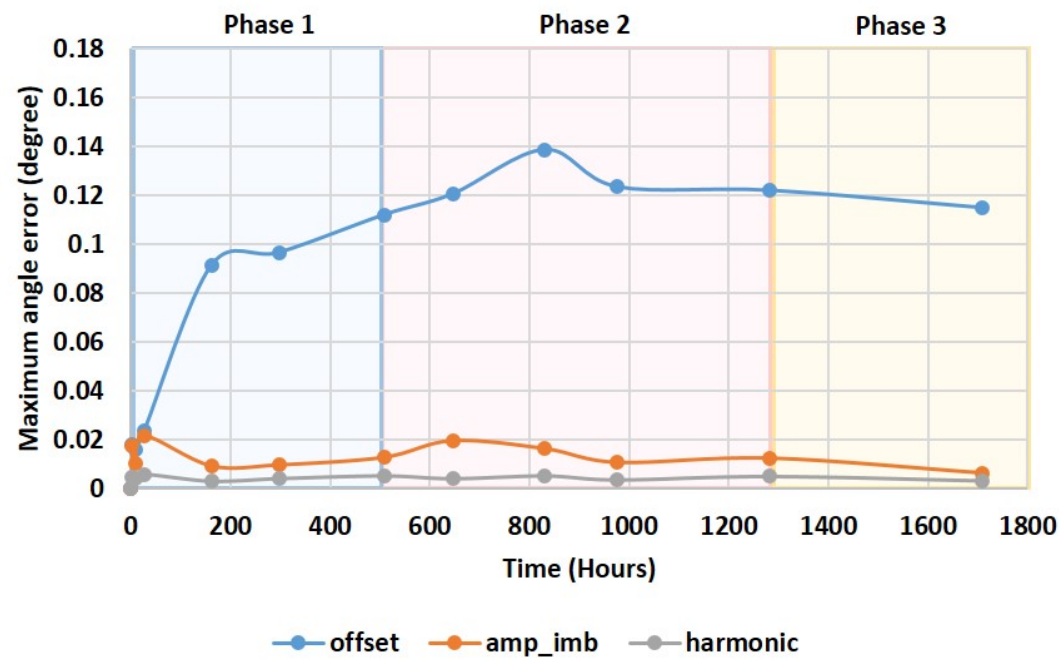

Figure 3.41: Drift of the angle errors in test group 3 during the second aging test.

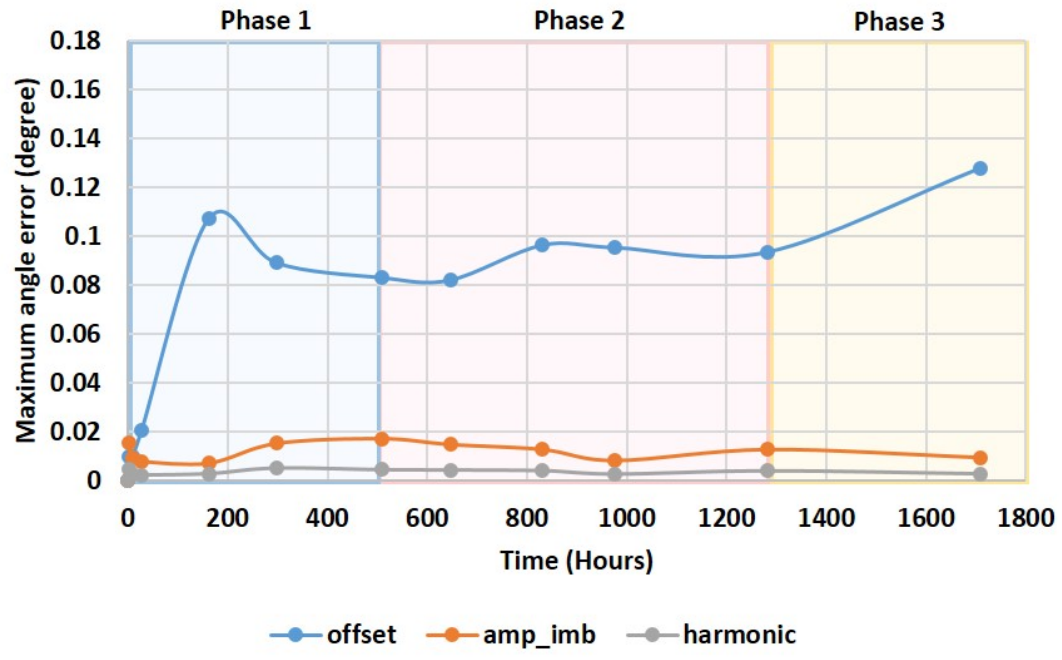

Figure 3.42: Drift of the angle errors in test group 4 during the second aging test.

Table 3.5: Drift of the angle errors in degrees at the end of the second aging test

\begin{tabular}{|c|c|c|c|}
\hline $\begin{array}{c}\text { Test } \\
\text { group } \sharp\end{array}$ & $\begin{array}{c}\text { Due to offset } \\
\text { voltage }\end{array}$ & $\begin{array}{c}\text { Due to amplitude } \\
\text { Imbalance }\end{array}$ & $\begin{array}{c}\text { Due to } \\
\text { harmonics }\end{array}$ \\
\hline 1 & 0.1020 & 0.0106 & 0.0026 \\
2 & 0.1602 & 0.0101 & 0.0037 \\
3 & 0.1148 & 0.0065 & 0.0033 \\
4 & 0.1279 & 0.0098 & 0.0029 \\
\hline
\end{tabular}


The offset voltage is the largest source of angle errors at the start of the sensor's life but it also shows the largest drift due to aging effects. The other two sources of angle errors show fairly constant variations over time.

\subsubsection{Performance degradation}

AMR sensors are widely used in automotive applications for angle measurements. However, the sensor performance is affected by undesirable parameters such as offset voltage, amplitude imbalance and additional harmonics; that are all included in the sensor outputs since the beginning of its lifetime. Therefore, compensation factors are often applied to reduce the effect of these parameters on the accuracy of the angle to be calculated. Until now, the offset voltage is the parameter which is mainly compensated for because it is the largest source of the angle error as confirmed in the previous section. The compensation factors have usually been calculated at the factory at the start of the sensor's life cycle, but they are not updated during its lifetime.

However, the results of the aging tests that have been performed in this research confirm that the sensor parameters drift over time, of which the offset voltage shows the largest drift. At the end of the second aging test, half of the bridges in the aged sensors show drifts of the offset voltages higher than $50 \mu \mathrm{V} / \mathrm{V}$ as shown in Figure 3.28. These results satisfy the current requirement that the drift of the offset voltage over thousand hours at high temperature should be less than $100 \mu \mathrm{V} / \mathrm{V}$ to guarantee an angular accuracy better than 1 degree over fifteen years [Isl10]. Nevertheless, in the future it is expected that a narrower tolerance band for drifting (few tens of $\mu \mathrm{V} / \mathrm{V}$ ) is required in order to have an angle accuracy better than $0.1^{\circ}$ in which case it will be necessary to compensate the offset voltage for aging effects.

The trend of the drift of the offset voltage can be described as higher at the start but then slowing down over time. This effect is most likely linked to stress relaxation, which in material science means a decrease in stress in response to the same amount of strain generated in the structure under specific conditions regarding time, temperature and other stress levels [Wel09], [Bus15]. It explains why the variation of the offset voltage decreases as relaxation occurs, as concluded earlier by Isler in [Isl10]. During the wafer processing of the sensor, a passivation layer is applied on top of the permalloy material as a protection layer; however, it is well-known for generating highly-compressive stress, which in this case affects the magnetic material. Furthermore, the cutting and polishing of the wafer required to finalise a functional device can induce stresses in the magnetic material as well. The annealing treatment performed during the sensor manufacturing is to saturate the grain size growth in the permalloy to achieve high magnetoresistance values [Boz35]. It helps to release the stress developed during the mechanical processing of the sensor, but it is not entirely eliminated. Therefore some stress is still present during the sensor operation.

Regarding the signal amplitudes, the drift of the values is also linked to changes 


\subsection{Conclusions}

in the properties of the permalloy material. The initial increments shown in Figures 3.31 and 3.32, can be due to the stress relaxation or because the grain growth in the permalloy is not completely saturated yet during the sensor manufacturing. This last effect also explains the initial tendency of the bridge resistances to decrease due to an initial improvement of the conductivity of the permalloy because of its grain growth. Iben reported in [Ibe03] similar results for the amplitude and the bridge resistances of AMR sensors used in tape storage drives, which were submitted to accelerated tests at temperatures below about $250{ }^{\circ} \mathrm{C}$. While comparing the results of the aged groups with group 5 used as reference it is clear that the amplitude synchronism factor $k$ presented in Figure 3.33 shows less variations linked to aging effects than the offset voltage.

With respect to the angle errors, the error due to the offset voltage shows the largest drift with a trend similar to the offset voltage. The other two undesired parameters exhibit smaller and more stable values over time, so they are considered second-order effects concerning the angular error.

Although a major part of the stress remaining from manufacturing is released at the start of the sensor operating life, it is still submitted to thermal stress during its lifetime. This due to the temperature cycling experienced by the cars and the different thermal expansion coefficients of the materials that the sensors are composed of. Therefore performance degradation will continue over time as shown in the results during phase 3 of the aging test.

\subsection{Conclusions}

This chapter has studied the aging effects on the performance of AMR sensors used for angle measurements. A set of accelerated degradation tests (ADT) focussed on studying the performance degradation rather than on estimating the lifetime of AMR sensors have been performed. Compared with previous studies such as reported in [Ibe03] and [Isl10], more parameters that affect the performance of the sensors have been considered including offset voltage, the amplitude of the sensor outputs, amplitude imbalance between them, bridge resistances and angle errors. In addition, the operating condition of the sensors was taken into consideration to define the stress conditions during the aging tests, which allows simulating more realistically the degradation process experienced by the sensors in real life. The results show that the sensor performance drifts over time.

The results obtained regarding the drift of the offset voltage agree with the results reported by Isler in [Isl10] for AMR sensors used in automotive applications. The results of the drift of the amplitude and the bridge resistances are consistent with the results presented by Iben in [Ibe03] for AMR sensors used in tape storage drives. Additionally, this research includes the study of the angle error over time as it is a critical parameter to determine the performance of AMR sensors. The offset voltage is the most significant source of angle error at the start of the sensor's life 
but also shows the largest drift due to aging effects. Although the drift observed allows complying with the current requirement of an angle accuracy better than $1^{\circ}$ over the sensor lifetime, this will change in the future as can be expected accuracy requirements in the order of $0.1^{\circ}$, especially with the current trend of autonomous cars. Then, the compensation of the offset voltage for aging effects will be required. The second largest source of angle error is the amplitude imbalance between the sensor outputs, which shows more constant values over time.

In the case of the offset voltage, Isler proposes in [Isl10] to use a longer annealing time in the factory to reduce the drift of the offset voltage during the sensor's lifetime. However, this implies an increase in manufacturing time and cost of the sensor, both critical aspects in the automotive industry. Therefore, we considered it is a better option to update the compensation factors calculated at the start of a sensor's life during its operational life. 


\section{References}

[AEC13] AEC (Automotive Electronic Council). "Guideline for Characterization of Integrated Circuits". Component Technical Committee, 2013.

[Ber08] B. Bernd. "Methods for Reliability Test Planning". Reliability in Automotive and Mechanical Engineering: Determination of Component and System Reliability, pp. 264-290, 2008.

[Boz35] R. Bozorth and J. Dillinger. "Heat treatment of magnetic materials in a Magnetic field. II. Experiments with Alloys". In Physics, volume 6, pp. 285-291, 1935.

[Bus15] K. Buschow. "Handbook of Magnetic Materials". volume 24, 2015.

[Che16] Z. Chen, S. Li, and E. Pan. "Optimal constant-stress accelerated degradation test plans using nonlinear generalized wiener process". In mathematical problems in engineering, pp. 1-11, 2016.

[Fel04] A. Felscher. "Programmable angle sensor KMA200. Aplication note". Technical report, Philips Semiconductors, 2004.

[Gon15] H. Goncalves. "http://www.onmyphd.com/?p=harmonics", 2015. Accessed 2015-08-06.

[Gor09] N. Gorjian, L. Ma, and M. Mittinty. "A review on degradation models in reliability analysis". In Proceedings of the 4 th world congress on engineering asset management, pp. 369-384, Sep 2009.

[Ibe03] I. Iben. "Head Reliability of AMR Sensors Based on Thermal Stress Tests". IBM J. Res. Dev., volume 47(4), pp. 415-428, Jul 2003.

[Isl10] M. Isler, B. Christoffer, and G. Schoer. "Optimisation of surface passivation for highly reliable angular AMR sensors". Physica Status Solidi (c), volume $7(2)$, pp. 436-439, 2010.

[Lab14] Labmate. "what is the difference between repeatability and reproducibility?" www.labmate-online.com, Jun 2014.

[Lar14] J. Lara and A. Chandra. "Position error compensation in quadrature analog magnetic encoders through an iterative optimization algorithm". In 40th Annual Conference of the IEEE Industrial Electronics Society, pp. 3043-3048, Oct 2014.

[Li02] Q. Li. Accelerated degradation test planing and optimization. Ph.D. thesis, University of Arizona, 2002.

[Liu10] M. Liu, Z. Ren, and Y. Fu. "The methodology of life prediction and validation of electronic products based on accelerated degradation testing". In 11th International Conference on Electronic Packaging Technology High Density Packaging, pp. 1053-1058, Aug 2010. 
[Moh10] S. H. Mohammadian, D. Ait-Kadi, and F. Routhier. "Quantitative accelerated degradation testing: Practical approaches". Reliability Engineering and System Safety, volume 95(2), pp. 149-159, 2010.

[NXP11] NXP. "KMZ49 datasheet". NXP semiconductors, 2011.

[Ted16] Tedpavlic. "http://www.tedpavlic.com/teaching/osu/ece209, Lissajous curves", 2016. Accessed 2016-12-06.

[Vas08] P. Vassiliou, A. Mettas, and T. El-Azzouzi. "Quantitative Accelerated Lifetesting and Data Analysis". In Handbook of Performability Engineering, pp. 544-557, 2008.

[Wan16] H. Wang, G. Wang, and F. Duan. "Planning of step-stress accelerated degradation test based on the inverse Gaussian process". In reliability engineering and system safety, volume 154, pp. 97-105, 2016.

[Wel09] B. Wellman. "Stress relaxation and creep". Technical TIDBITS, volume 2, 2009 .

[Wik15] Wikipedia. "https://en.wikipedia.org, List of trigonometric identities", 2015. Accessed 2015-08-06.

[Yum07] B. Yum, H. Lim, and S. Seo. "Planing performance degradation testa review". In International Journal of Industrial Engineering: Theory, Applications and Practice, volume 14, pp. 372-381, 2007.

[Zam15] A. Zambrano and H. G. Kerkhoff. "Determination of the aging offset voltage of AMR sensors based on accelerated degradation test". In 20th IEEE International Mixed-Signals Testing Workshop (IMSTW), pp. 1-5, June 2015.

[Zam16] A. Zambrano and H. G. Kerkhoff. "Determination of the drift of the maximum angle error in AMR sensors due to aging". 21st IEEE International Mixed-Signal Testing Workshop (IMSTW), pp. 1-5, July 2016.

[ZVE13] ZVEI (Zentralverband Elektrotechnik und Elektronikindustrie). "Handbook for Robustness Validation of Automotive Electrical/Electronic modules". June 2013. 


\title{
Chapter 4
}

\section{Self-X properties of AMR sensors}

\begin{abstract}
In chapter 3, it has been concluded that in the future aging compensation will be required in AMR sensors used for angle measurements. In this chapter, it is proposed to add self-X properties to allow a sensor system to handle the performance degradation of the sensor. This includes self-monitoring to determine the performance of the sensor and self-calibration to execute the required adjustments in case the performance decreases over time. In this way, it will be possible to satisfy the ever increasing dependability requirements of automotive applications now and in the future.
\end{abstract}

\subsection{Introduction}

The self-X approach allows improving the dependability of a system by providing the system components such as processors, amplifiers and sensors with capabilities to perform certain functions on its own. From the perspective of the sensors, self-X features can improve flexibility, accuracy as well as reduce their vulnerability to deviations and drift resulting from manufacturing and environmental changes. Features such as self-monitoring and self-calibration can be used to monitor and recover the sensor performance [Joh11a]. However, it is highly desirable that this is performed with a minimum of computational effort and a minimum of additional hardware; this is especially relevant in the automotive industry which has limitations regarding cost, space and weight of the components of the systems [Von06].

The self-monitoring feature provides the capability to monitor the sensor to guaranty that it provides the best performance under optimal conditions without using expensive external hardware for test or calibration. Without monitoring, the system is unaware of sensor malfunctioning and hence considers that its output is always correct. With regard to self-calibration, it allows the sensor to perform

Part of this chapter has been presented at the 2015 IEEE International Instrumentation and Measurement Technology Conference (I2MTC) [Zam15], the 22nd IEEE International Symposium on On-line Testing and Robust System Design (IOLTS) [Zam16b], the 2016 IFIP/IEEE International Conference on Very Large Scale Integration (VLSI-SOC) [Zam16a] 
adjustments to recover its performance in case that it decreases over time. This is usually achieved by compensating undesired effects in the sensor outputs at the signal-conditioning or processing level [Joh11a], [Joh11b].

AMR sensors for angle measurements are generally set to operate under optimal conditions at the start of the sensors' lifetime. This usually means that compensation factors are determined to reduce the effect of the undesirable parameters included in the sensor outputs [Die00]. Because the offset voltage is the most significant contributor to the angle error as shown in chapter 3, this is the parameter that is usually compensated so far. Although the sensor shows performance degradation due to wearing and aging effects, its performance remains within the tolerance band currently permitted; therefore up to now the compensation factors are usually not updated over time [Isl10]. However, it has been concluded in chapter 3 that in the future it will be necessary to also compensate for aging effects, especially in the case of the offset voltage.

The discussed research in this chapter is focussed on proposing self-X features that allow the sensor to handle the performance degradation over time. Next, a self-monitoring approach is discussed in detail which is aimed to verify the angle error. This is a critical parameter in the performance of AMR sensors used for angle measurements. Furthermore, a self-calibration approach is proposed to update the compensation factors calculated at the beginning of the sensors' life.

The remainder of the chapter is organised as follows. In section 4.2 the selfmonitoring feature is proposed along with the validation results. Section 4.3 presents the self-calibration approach including implementation results. Finally, conclusions are provided in section 4.4 .

\subsection{Self-Monitoring}

\subsubsection{Introduction}

As mentioned above, the self-monitoring property allows monitoring the performance of a system to verify whether it is operating at optimum conditions over time. This is usually carried out by observing a parameter from which the performance of the system can be inferred. In the case of AMR sensors used for angle measurements, the inaccuracy of the angle calculated is a suitable parameter for monitoring the performance of the sensors. Inaccuracy means errors in the angle calculation, mainly due to undesirable parameters included in the sensor outputs, as explained in chapter 2 .

Figure 4.1 compares the set magnetic angles versus the calculated angles using ideal sensor outputs as well as sensor outputs in which undesirable parameters are also included. In the case the bridge outputs do not show any imperfection, a perfectly straight line is obtained. This is not the same when the sensor outputs show non-perfect sinusoidal signals. The more significant the difference between 


\subsection{Self-Monitoring}

these two lines, the more angle errors occur.

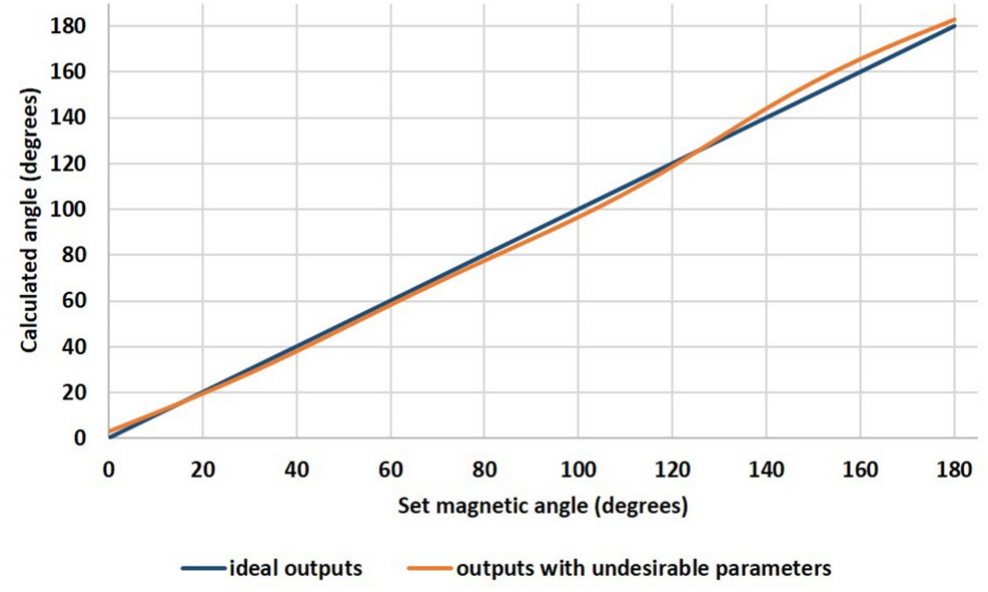

Figure 4.1: Set magnetic angles versus calculated angles from ideal and actual sensor outputs.

The angle errors due to the undesired parameters included in the sensor outputs show sinusoidal characteristics as presented in Table 3.2 of chapter 3 [Lar14]. For monitoring purposes their maximum values are of primary interest, and therefore a methodology to calculate these maximums online is proposed. This includes a set of equations that take into consideration the following five conditions of the sensor outputs.

Condition 1: the output voltages of bridge 1 and 2 should be a cosine and a sine signal that depend on the angle to be measured, as shown in Figure 4.2.

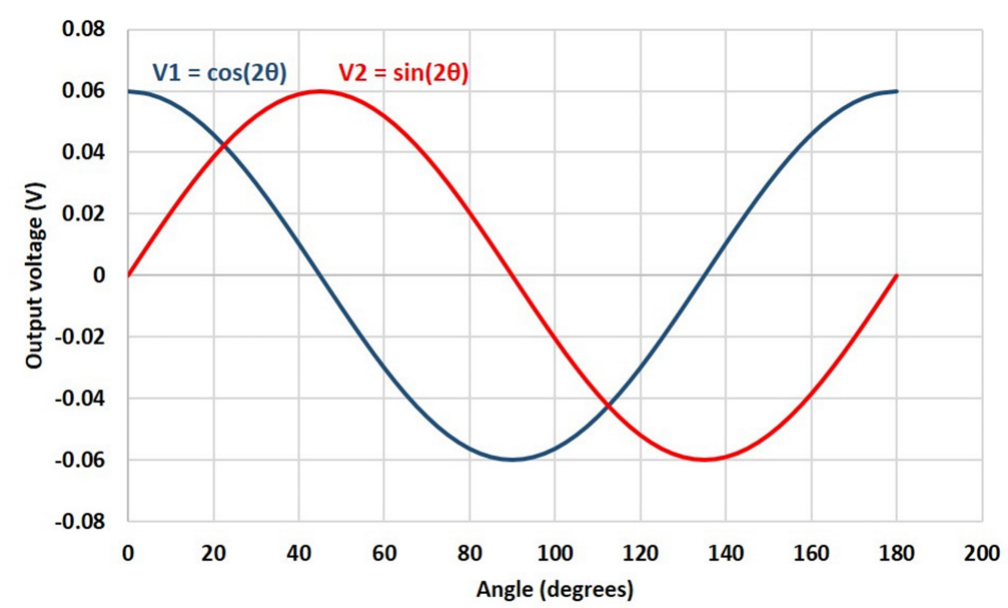

Figure 4.2: Expected output signals of an AMR sensor. $V 1$ and $V 2$ represent the output signals of bridge 1 and 2 respectively. 
Then, the magnetic angle $(\theta)$ is calculated from the output voltages of the bridges $\left(V 1, V^{2}\right)$, as shown in Equation (4.1).

$$
2 \theta=\arctan \left(\frac{V 2}{V 1}\right)
$$

Based on the sinusoidal characteristics of the sensor outputs and the Pythagorean identity shown in Equation (4.2) for a unit circle [Bra17], the sum of the square of the output voltages of the bridges should be a fixed value regardless the angle of the magnetic field, as shown in Equation (4.3). This value is equal to the square of the amplitude of the sinusoidal signals, which in electrical terms can be considered as a DC voltage.

$$
\begin{gathered}
\sin (\theta)^{2}+\cos (\theta)^{2}=1 \\
(V 1)^{2}+(V 2)^{2}=\text { fixed }_{\text {value }}(D C)
\end{gathered}
$$

Condition 2: the undesirable parameters included in the sensor outputs generate variations in the sum of the square of the bridge outputs as shown in Figure 4.3. The results presented are obtained with ideal and actual sensor outputs.

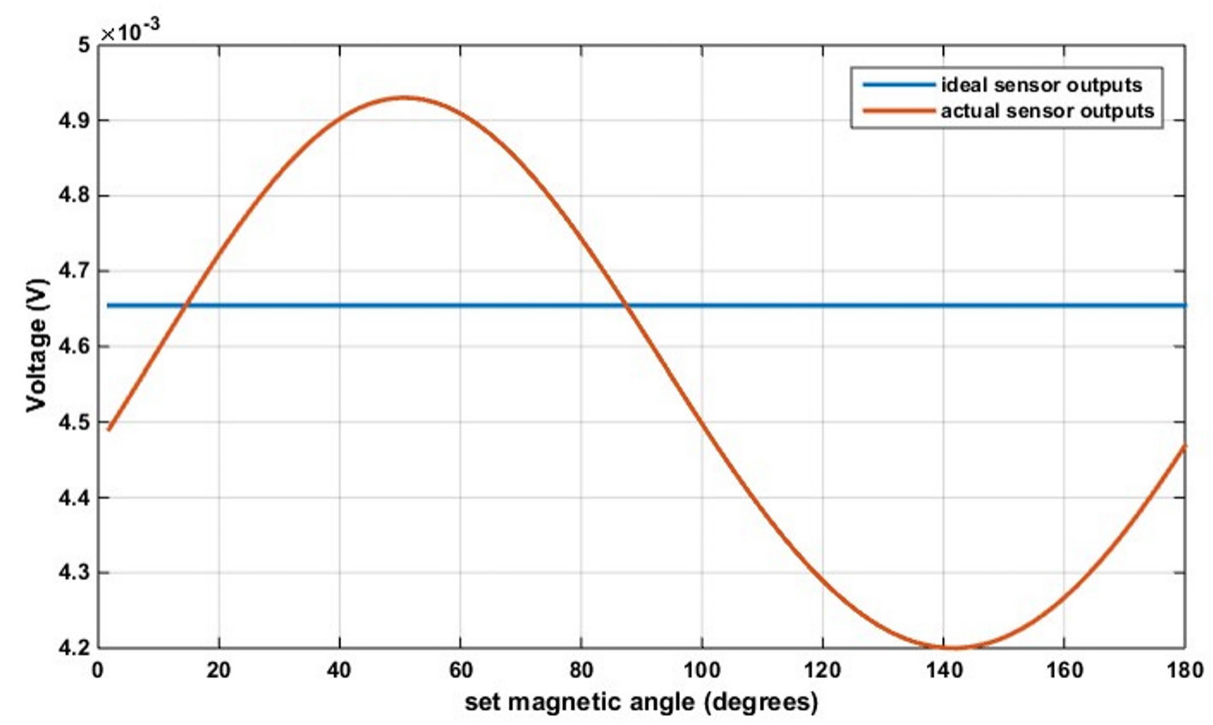

Figure 4.3: Signals obtained from the sum of the square of ideal and actual bridge outputs. 
As a result, Equation (4.3) cannot be described as a fixed value (DC voltage), but as a DC voltage $(D C)$ plus an $\mathrm{AC}$ voltage $(A C)$, as shown in Equation (4.4). The $\mathrm{AC}$ voltage is linked to the error in the angle calculation.

$$
(V 1)^{2}+(V 2)^{2}=D C+A C
$$

Condition 3: the $A C$ and $D C$ component in Equation (4.4) could be combined with the Equation (4.1) used to calculate the magnetic angle. The resulting Equation (4.5) includes the Root-Mean-Squared (RMS) value of the $A C$ component of Equation (4.4) in the numerator and the $D C$ component in the denominator.

$$
\text { angle }_{\text {error }}=\arctan \left(\frac{R M S_{A C}}{D C}\right)
$$

Condition 4: if the sensor outputs are two perfect sinusoidal signals, then the numerator in Equation (4.5) becomes zero and the equation indicates zero degrees of error. On the other hand, if the $A C$ component is not zero, the resulting value of Equation (4.5) is related to the angle error.

Condition 5: the $A C$ component is defined by the most significant source of angle error present in the sensor outputs, which generate different error signals, as shown in Table 3.2 of chapter 3. Figure 4.4 shows the $A C$ component obtained from the sum of the square of the sensor outputs in three different cases.

Case A shows the $A C$ component obtained from the sensor outputs without any compensation being the offset voltage the largest source of angle error, which is characterized by a sine signal with the same frequency as the sensor. In case $\mathrm{B}$ the output voltages with offset voltage compensated have been used, so the $\mathrm{AC}$ component is defined by the amplitude imbalance. This shows a sine behaviour with a frequency equal to the double of the sensor frequency and a smaller amplitude compared to case $\mathrm{A}$. This is because the angle error due to the amplitude imbalance is smaller than the angle error due to offset voltages as presented in chapter 3. Case $\mathrm{C}$ presents the $\mathrm{AC}$ component obtained from the sensor outputs with offset voltage and amplitude imbalance compensated, which shows a smaller amplitude than the previous cases because the additional harmonics are the largest source of angle error. 

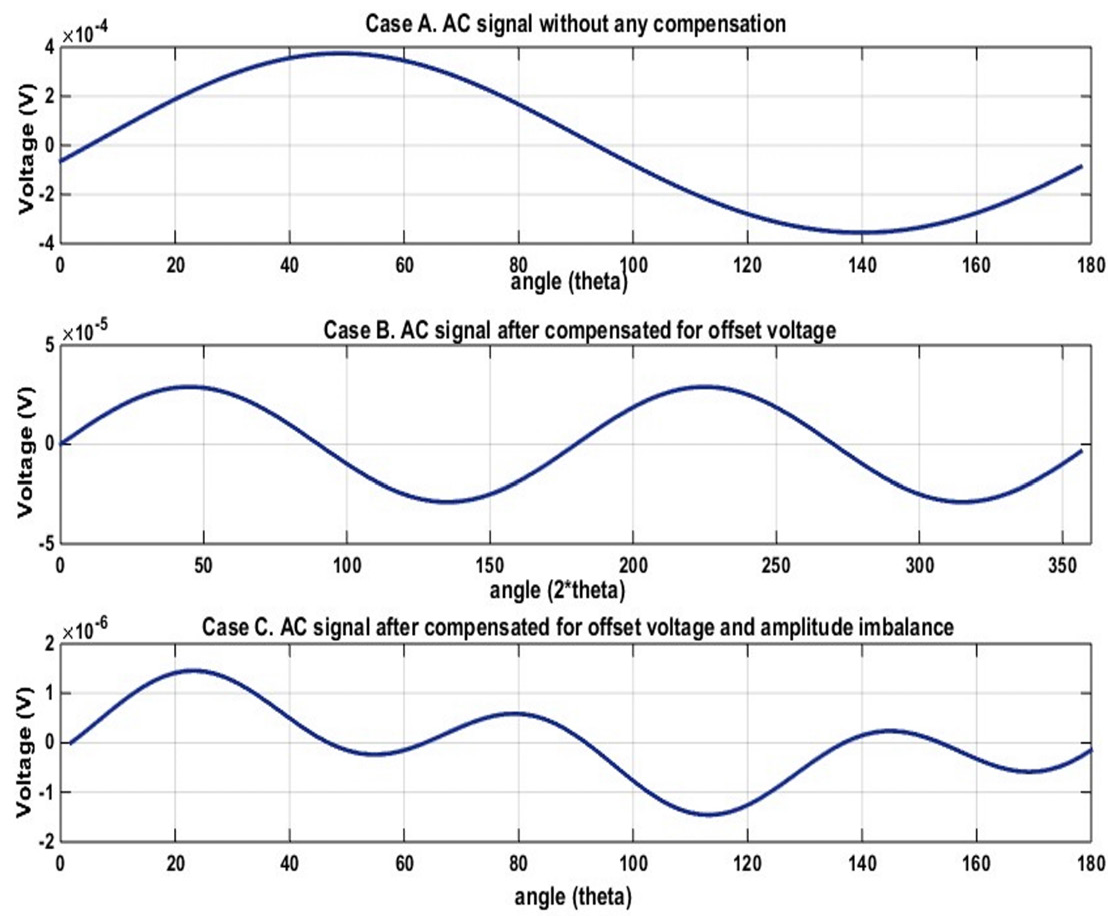

Figure 4.4: Signals obtained from the sum of the square of the bridge outputs in three different cases.

Based on the five assumptions listed above regarding the sensor outputs, Equations (4.6), (4.7) and (4.8) have been defined to calculate the maximum angle errors due to offset voltage, amplitude imbalance and harmonics respectively. In order to obtain the maximum value of each error source, Equation (4.5) should be multiplied by a factor linked to the angle at which the respective angle error is maximum. As mentioned above, the angle error due to offset voltage and amplitude imbalance show sinusoidal signals in which the maximum is at $90^{\circ}$ taking into consideration the detected angle by the sensor or a magnetic angle of $45^{\circ}$. With regard to the additional harmonics, the factor is related to the cosine signal shown in the first component of the signal presented in Table 3.2 for harmonics.

Now the equations for the maximum angle error due to each error source will be provided:

- Equation for the maximum angle error due to offset voltage:

$$
\text { angle }_{\text {error } 1}=\arctan \left(\frac{R M S\left(R 1_{A C}\right)}{R 1_{D C}}\right) * \frac{\sin \left(45^{\circ}\right)}{2}
$$

where:

$$
R 1=(V 1)^{2}+(V 2)^{2}
$$


$V 1$ and $V 2$ represent the sensor outputs without any compensation.

- Equation for the maximum angle error due to amplitude imbalance:

$$
\text { angle }_{\text {error } 2}=\arctan \left(\frac{R M S\left(R 2_{A C}\right)}{\left.R 2_{D C}\right)}\right) * \frac{\sin \left(45^{\circ}\right)}{2}
$$

where:

$$
R 2=(V 1 c o m p)^{2}+(V 2 c o m p)^{2}
$$

$V 1$ comp and $V 2 c o m p$ represent the sensor outputs after offset voltage compensation.

- Equation for the maximum angle error caused by harmonics:

$$
\text { angle }_{\text {error } 3}=\arctan \left(\frac{R M S\left(R 3_{A C}\right)}{\left.R 3_{D C}\right)}\right) * \cos \left(22.5^{\circ}\right)
$$

where

$$
R 3=(V 1 a i)^{2}+(V 2 a i)^{2}
$$

$V 1 a i$ and $V 2 a i$ represent the sensor outputs after compensating for offset voltage as well as amplitude imbalance.

The last three equations are aimed to calculate the maximum angle error due to the various sources. However, all of them include the ARCTAN function that requires a highly iterative process to calculate its value, meaning more time and processing resources of the system. Therefore, it is not the preferred option to be implemented online for automotive applications. Next, a simplified version of these equations is presented which are based on the fact that for small angles the ARCTAN is approximately equal to the angle in radians. This applies for small angles as is the case for the angle errors in AMR sensors as shown in Figure 3.11 presented in chapter 3 , in which commercial sensors show values smaller than 2 degrees for which the assumption is valid. In the multiplication factors, the conversion from radians to degrees has also been included to obtain the angle error in degrees.

By doing so, Equation (4.6) for the maximum angle error due to offset voltage reduces to:

$$
\text { angle }_{\text {error } 1}=\frac{R M S\left(R 1_{A C}\right)}{R 1_{D C}} * 20
$$

In a similar way, Equation (4.7) for the maximum angle error due to amplitude imbalance reduces to: 


$$
\text { angle }_{\text {error } 2}=\frac{R M S\left(R 2_{A C}\right)}{R 2_{D C}} * 20
$$

And finally, Equation (4.8) of the maximum angle error due to harmonics is simplified to:

$$
\text { angle }_{\text {error } 3}=\frac{R M S\left(R 3_{A C}\right)}{R 3_{D C}} * 53
$$

The simplified version of the proposed equations is more suitable to implement online monitoring of the sensor performance during its lifetime. In this way, it is possible to take further actions such as updating of compensation factors if required.

\subsubsection{MATLAB implementation}

The proposed equations to calculate the maximum angle errors due to the undesired parameters included in the sensor outputs have been verified by Matlab implementation. As input data, the voltages obtained from an analytical model of the sensor implemented in MATLAB that is included in Appendix B have been used, but also voltages measured in commercial sensors. The verification has been done by calculating the percentage of error, a parameter commonly used to verify experimental data. It is defined as the difference between an approximate value and an exact value as a percentage of the exact value, as shown in Equation (4.12) [App16]. In this research, the approximate values refer to the results obtained with the proposed equations and the exact values were calculated as shown in the Equations (4.13), (4.14) and (4.15).

Percentage of error $=\frac{\mid \text { approximate value }- \text { exact value } \mid}{\mid \text { exact value } \mid} * 100 \%$

- Maximum angle error due to offset voltage $\left(M A E_{1}\right)$ :

$$
M A E_{1}=\operatorname{maximum}(\text { angle } 1-\text { angle } 2)
$$

where: angle1 represents the angles calculated with the sensor outputs without any compensation and angle2 the angles calculated with the sensor outputs after offset voltage compensation.

- Maximum angle error due to amplitude imbalance $\left(M A E_{2}\right)$ :

$$
M A E_{2}=\operatorname{maximum}(\operatorname{angle} 2-\text { angle } 3)
$$


where: angle2 represents the angles calculated with the sensor outputs after offset voltage compensation and angle3 the angles calculated with sensor outputs with offset voltage and amplitude imbalance compensated.

- Maximum angle error due to harmonics $\left(M A E_{3}\right)$ :

$$
M A E_{3}=\operatorname{maximum}(\text { angle } 3-\text { angle } 4)
$$

where: angle3 represents the angles calculated with sensor outputs with offset voltage and amplitude imbalance compensated and angle4 the angles calculated with sensor outputs with the three sources of angle error compensated.

A hundred cases that represent 100 AMR sensors with different values regarding offset voltage, amplitude imbalance and harmonics have been simulated. Table 4.1 presents the arithmetic mean and standard deviation (std) of the percentage of error (Equation (4.12)) of these cases for the three sources of angle errors. The smaller the percentage of error the more accurate the value has been calculated. Therefore, the angle error due to amplitude imbalance shows the most accurate results, as it presents the smallest mean and standard deviation values. The most significant inaccuracy is occurring in the harmonics with $14 \%(8 \%+6 \%)$. However, overall the proposed equations provide a good estimation of the angle errors.

If the values obtained with the equations that include the ARCTAN function and the simplified version are compared, both present very similar results for offset voltage and harmonics, but not for amplitude imbalance. Nevertheless, for this error, the simplified version gives a maximum error of $1.77 \%(1.32 \%+0.45 \%)$ which means these are still accurate results. Hence it is possible to implement the simplified equations version on-chip because it demands less processing resources of the system.

Table 4.1: The arithmetic mean and standard deviation (std) of the percentage of errors obtained for each source of angle error in 100 simulated cases of AMR sensors.

\begin{tabular}{|c|c|c|c|c|c|c|}
\hline \multirow{3}{*}{$\%$} & \multicolumn{2}{|c|}{ offset voltage } & \multicolumn{2}{c|}{ amplitude imbalance } & \multicolumn{2}{c|}{ harmonics } \\
\cline { 2 - 7 } Error & proposed & simplified & proposed & simplified & proposed & simplified \\
& equation & version & equation & version & equation & version \\
& Eq.(4.6) & Eq.(4.9) & Eq.(4.7) & Eq.(4.10) & Eq.(4.8) & Eq.(4.11) \\
\hline mean & 4.85 & 4.42 & 0.27 & 1.32 & 8.01 & 8.03 \\
std & 5.63 & 5.04 & 0.90 & 0.45 & 6.23 & 6.29 \\
\hline
\end{tabular}

The proposed Equations (4.6) and (4.9) for the maximum angle error due to offset voltage have also been verified with data obtained from the 24 commercial AMR sensors (KMZ49) used in the second aging test explained in chapter 3. Table 
4.2 shows the arithmetic mean and standard deviation (std) of the percentage of errors calculated as explained before. The results show that the percentage of error obtained with the simplified version of the equation is $3.15 \%(1.94 \%+1.21 \%)$, which confirms that the equation provides a good estimation of the maximum of this source of angle error.

Table 4.2: The arithmetic mean and standard deviation (std) of the percentage of error obtained for the maximum angle error due to offset voltage in 24 commercial sensors.

\begin{tabular}{|c|c|c|}
\hline \multirow{2}{*}{ \% Error } & \multicolumn{2}{|c|}{ offset voltage } \\
\cline { 2 - 3 } & $\begin{array}{c}\text { proposed equation } \\
\text { Eq.(4.6) }\end{array}$ & $\begin{array}{c}\text { simplified version } \\
\text { Eq.(4.9) }\end{array}$ \\
\hline mean & 2.19 & 1.94 \\
std & 1.71 & 1.21 \\
\hline
\end{tabular}

The data obtained from commercial AMR sensors also includes an error due to the measurement setup. In our measurements, this error is smaller than the error as a result of offset voltage but larger than the error from amplitude imbalance and the harmonic components. From the angle errors, it has been determined that in average the angle error due to the measurement setup is 0.6 degrees, while the average value of the maximum angle error from amplitude imbalance is 0.17 degrees and for the maximum angle error resulting from harmonics is 0.06 degrees. Therefore, the previous data is not suitable to verify the proposed equations for these two sources of angle errors. However, this does not invalidate the proposed equations, but implies that the angle error because of the measurement setup should be smaller than the angle errors from the undesirable parameters included in the output voltages of the sensors.

The proposed equations allow online monitoring of the sensor performance during its lifetime. However, they cannot be applied in real-time because the sensor outputs at different angles during one period of the sinusoidal signals are required as input. Nevertheless, it has been shown in chapter 3 that the angle error drifts slowly over time, so it is possible to verify the angle errors from time to time, every time the car is turned on or in case some event occurs. For example, the offset voltage generates the largest angle error and also shows the largest drift over time, so its variation can be used as an event that sets a flag to indicate that the maximum angle error should be verified in the system.

As to be explained in section 4.3.2, the offset voltage consists of two parts, one per each half bridge like is the case of the output voltages used to calculate the angle. The addition of the two components of the offset voltage is easily obtained by subtracting the voltages of two resistances oriented in the same direction inside the Wheatstone bridge, as is the case of resistances $\mathrm{R} 1$ and $\mathrm{R} 3$ in Figure 4.7. If 
this addition shows large variations, it means that the offset parts and the offset voltage also changes. Figure 4.5 presents as an example, the drift of the offset voltage and the variation of the addition of the two offset parts along the read points in bridge 1 for one of the aged sensors reported in chapter 3. The result shows that the larger the drift of the offset voltage at the start, the larger is the variation of the addition between the read points; then the variation decreases as the drift slows down. If a system flag is configured to indicate variations of the addition larger than $20 \mu \mathrm{V} / \mathrm{V}$, it will be set to 1 to notify the system that an event occurred between the read points 4 and 5, 5 and 6, 7 and 8, and the maximum angle error should be verified.

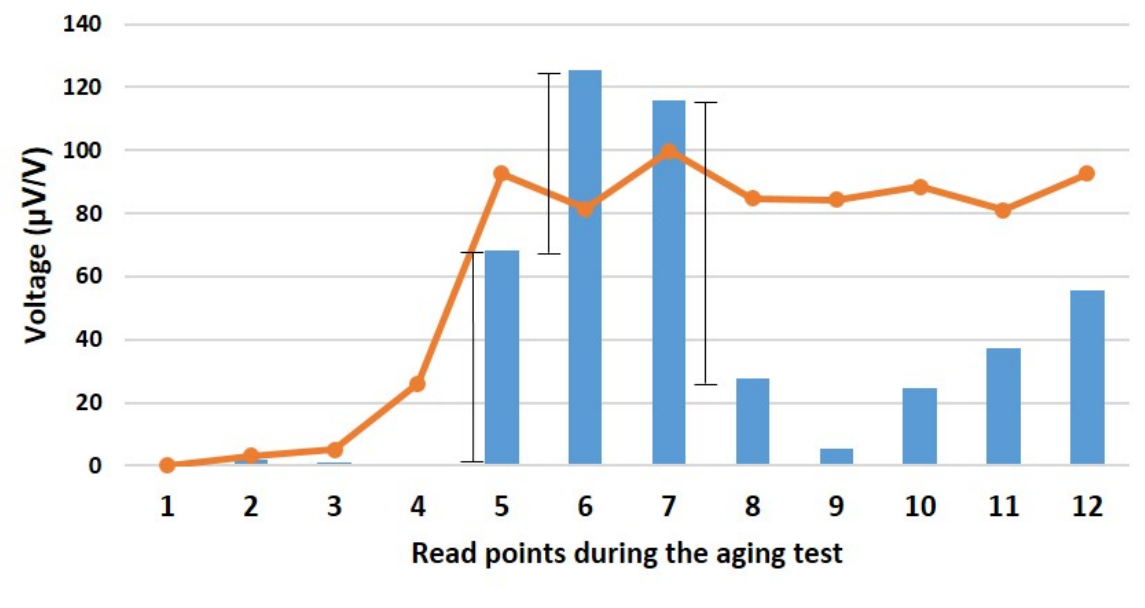

addition of offset components between measurements $\multimap$ offset voltage

Figure 4.5: The drift of the offset voltage and variation of the addition of its components along the read points in bridge 1 for one of the aged sensors reported in chapter 3 .

In summary, the proposed equations to calculate online the maximum angle errors due to the undesired parameters included in the sensor outputs are based on the trigonometry relationship of the sensor outputs. The variation (AC component) obtained in the addition of the square of the output voltages is defined by the largest source of error present. As shown in chapter 3, the offset voltage generates the largest angle error at the beginning of the sensor life but also shows the largest drift due to aging effects. Therefore if processing resources of the system are limited, in practice Equation (4.9) can be applied to verify this angle error if the system flag for variation of the addition of the two offset parts so indicate. The other two angle errors due to amplitude imbalance and harmonics can be estimated for example if the performance degradation of each source of angle error is of interest. Based on the value of the maximum angle error and the accuracy required for the angle calculation it can be determined if an update of the compensation factors is needed, which can be accomplished with the self-calibration method proposed in the following section. 


\subsection{Self-Calibration}

\subsubsection{Introduction}

The self-calibration property allows the system to recover its performance in the case degradation occurs over time by performing its own adjustments under operating conditions. This is especially useful in safety-critical systems that demand high performance during their lifetime [Joh11a], [Liu00]. In AMR sensors for angle measurements, an initial calibration procedure is usually performed under factory conditions to adjust the sensor performance by compensating for the offset voltage. Most of the compensation methods proposed so far are aimed to be used under these conditions as explained in chapter 2; hence they are not suitable to be implemented as a self-calibration feature. This research proposes a method to compensate for offset voltage over time under the operational conditions of the sensor, but it is also possible to compensate for amplitude imbalance that is the second largest source of angle error in AMR sensors. In the following, this method is explained in detail.

\subsubsection{Proposed method}

The proposed compensation method is based on the phase relationship between the two sinusoidal signals of the sensor outputs. Because of the 90-degree phase difference between these two sinusoidal signals, the Lissajous curve obtained with the sensor outputs should result in a circle with a radius that represents the signal amplitudes and the centre representing the offset voltages. In theory, the Lissajous curve should be a perfect circle centred at $(0,0)$, but this is not the case with actual sensor outputs, in which case the curve tends to be an imperfect circle whose centre is displaced from the origin. Nevertheless, some corrections can be applied to improve the characteristics of the Lissajous curve.

Figure 4.6 presents a schematic diagram of the proposed compensation method. Figure 4.6a represents the Lissajous curve obtained with actual sensor outputs without any compensation. Then, compensating first for offset voltage it is possible to shift the centre of the Lissajous curve to the origin, as shown in Figure 4.6b. After that, compensating for amplitude imbalance allows obtaining a Lissajous curve closer to a perfect circle as presented in Figure 4.6c. This last Lissajous curve implies a higher accuracy in the angle calculation as compared with the initial curve in Figure 4.6a.

Our proposed compensation method defines a set of equations to calculate the compensation factors for offset voltage and amplitude imbalance during the sensor lifetime. The equations for the offset voltages are based on a set of four assumptions listed below, which are presented according to the voltages shown for each bridge in Figure 4.7. 


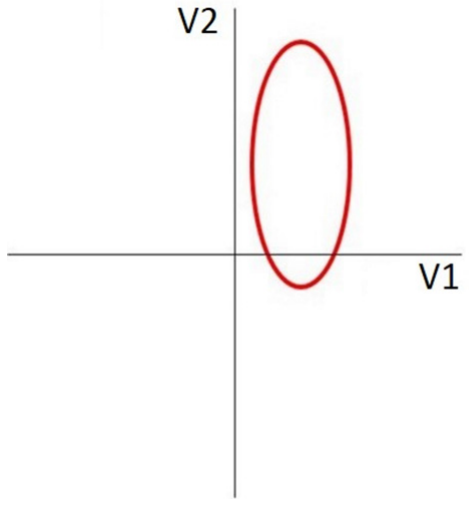

(a) Without any compensation.

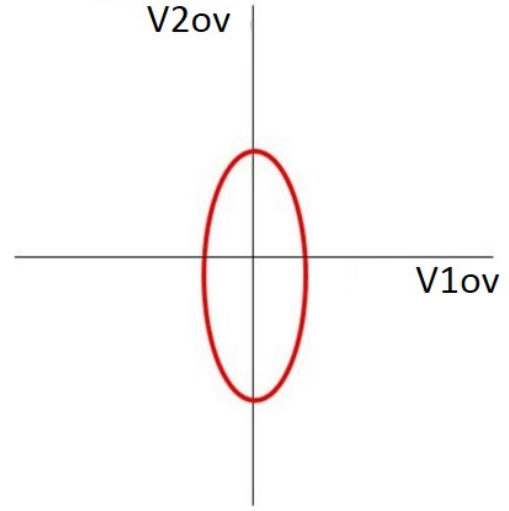

(b) Offset voltage compensated.

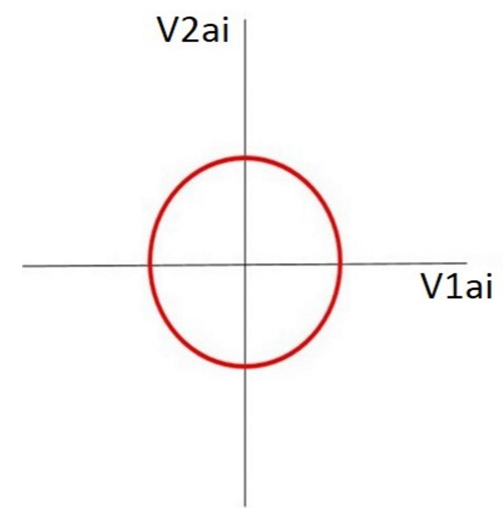

(c) Offset voltage and amplitude imbalance compensated.

Figure 4.6: Schematic diagram of the proposed compensation process in terms of the effect on the Lissajous curve. V1, V2 ; V1ov, V2ov, V1ai, V2ai represent the voltages used at each phase. 


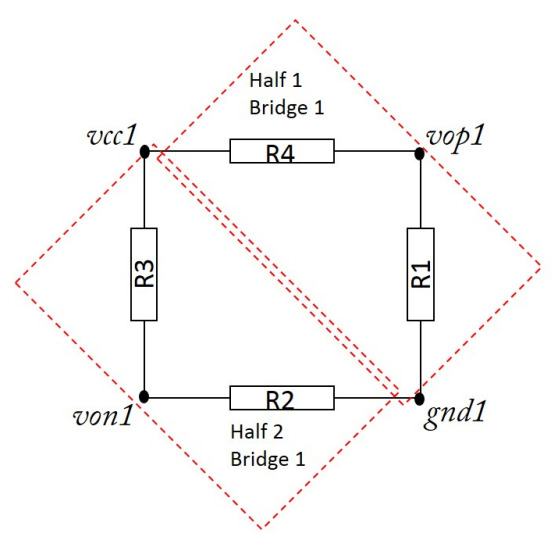

$$
\begin{aligned}
& V_{R 1}=\frac{V c c 1}{2}+\frac{A M R_{v 1}}{2}+o f f p 1 \\
& V_{R 2}=\frac{V c c 1}{2}-\frac{A M R_{v 1}}{2}+o f f n 1 \\
& V_{R 3}=\frac{V c c 1}{2}+\frac{A M R_{v 1}}{2}-o f f n 1 \\
& V_{R 4}=\frac{V c c 1}{2}-\frac{A M R_{v 1}}{2}-o f f p 1
\end{aligned}
$$

with $V c c 1$ : voltage of the power supply; $A M R_{v 1}$ : voltage due to magnetoresistance effect; off 1 and of f $n 1$ : offset voltage components; gnd1: ground 1

(a) Bridge 1 of an AMR sensor.

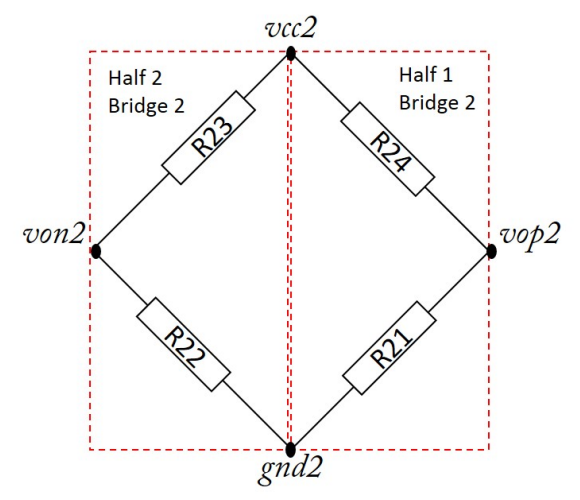

$$
\begin{aligned}
& V_{R 21}=\frac{V c c 2}{2}+\frac{A M R_{v 2}}{2}+o f f p 2 \\
& V_{R 22}=\frac{V c c 2}{2}-\frac{A M R_{v 2}}{2}+o f f n 2 \\
& V_{R 23}=\frac{V c c 2}{2}+\frac{A M R_{v 2}}{2}-o f f n 2 \\
& V_{R 24}=\frac{V c c 2}{2}-\frac{A M R_{v 2}}{2}-o f f p 2
\end{aligned}
$$

with $V c c 2$ : voltage of the power supply; $A M R_{v 2}$ : voltage due to magnetoresistance effect; off 2 and offn2: offset voltage components; gnd2: ground 2.

(b) Bridge 2 of an AMR sensor.

Figure 4.7: Schematic of the Wheatstone bridge 1 (a) and bridge 2 (b) of an AMR sensor with the respective voltages included. 


\subsection{Self-Calibration}

Assumption 1: the magnetic angle is calculated from the voltages in the bridge outputs as shown in Equation (4.16). In theory, these voltages should be equal to the voltage because of magnetoresistance effect AMRv1 and AMRv2 of bridge 1 and 2 respectively, as is also depicted in Equation (4.16). However, the actual sensor outputs include additional offset voltages (offset1, offset2), as shown in Equations (4.17) and (4.18).

$$
\begin{aligned}
2 \theta=\arctan \left(\frac{V 2}{V 1}\right) & =\arctan \left(\frac{\operatorname{vop} 2-\operatorname{von} 2}{\operatorname{vop} 1-\operatorname{von} 1}\right)=\arctan \left(\frac{A M R v 2}{A M R v 1}\right) \\
V 1 & =\operatorname{vop} 1-\operatorname{von} 1=A M R v 1 \pm \text { offset } 1 \\
V 2 & =\text { vop } 2-\operatorname{von} 2=A M R v 2 \pm \text { off set } 2
\end{aligned}
$$

Assumption 2: Equations (4.19) and (4.20) show that each offset voltage consists of one part per half-bridge.

$$
\begin{aligned}
& \text { offset } 1=\text { offp } 1-\text { off } n 1 \\
& \text { offset } 2=\text { of } f p 2-\text { of } f n 2
\end{aligned}
$$

Assumption 3: in theory, the resistances oriented in the same direction inside the Wheatstone bridge (e.g. R1 \& R3 in bridge 1) must show the same voltage value as they detect the same magnetic angle. Therefore their voltage subtraction should be equal to zero, however, the actual value is equal to the addition of the two parts of the offset voltage. Equations (4.21) and (4.22) show the voltage difference over the resistances (R1 \& R3) in bridge 1 and (R21 \& R23) in bridge 2, shown in Figure 4.7.

$$
\begin{gathered}
V_{R 1}-V_{R 3}=\text { off } f 1+\text { off } n 1 \\
V_{R 21}-V_{R 21}=\text { off } f 2+\text { of } f n 2
\end{gathered}
$$

Assumption 4: the Lissajous curve obtained from the voltages at the sensor outputs should be a circle in the ideal case. Equation (4.23) shows the circle equation, in which $x, y$ represent a circle point on the XY coordinate axis; $x c, y c$ the circle centre and $r$ denotes the radius. This assumption is valid because the amplitude imbalance between the sensor outputs is not large, as shown by the characterization results of a set of commercial AMR sensors presented in chapter 3.

$$
(x-x c)^{2}+(y-y c)^{2}-r^{2}=0
$$


Taking the assumptions presented above into consideration, Equations (4.24) and (4.25) have been derived to calculate what can be defined as instant values of the offset voltages. With these equations and a set of sensor outputs at three different angles $\left(V 1_{a}, V 2_{a} ; V 1_{b}, V 2_{b} ; V 1_{c}, V 2_{c}\right)$ shown in Figure 4.8, one set of off1 and off2 is calculated. Then with another set of sensor outputs $\left(V 1_{a^{\prime}}, V 2_{a^{\prime}} ; V 1_{b^{\prime}}, V 2_{b^{\prime}} ; V 1_{c^{\prime}}, V 2_{c^{\prime}}\right)$ defined by the shift $\Delta \varphi$, a new set of off 1 and off 2 is calculated. This process is repeated several times using voltages that belong to one period of the sinusoidal signals in the sensor outputs $(0,2 \pi)$.

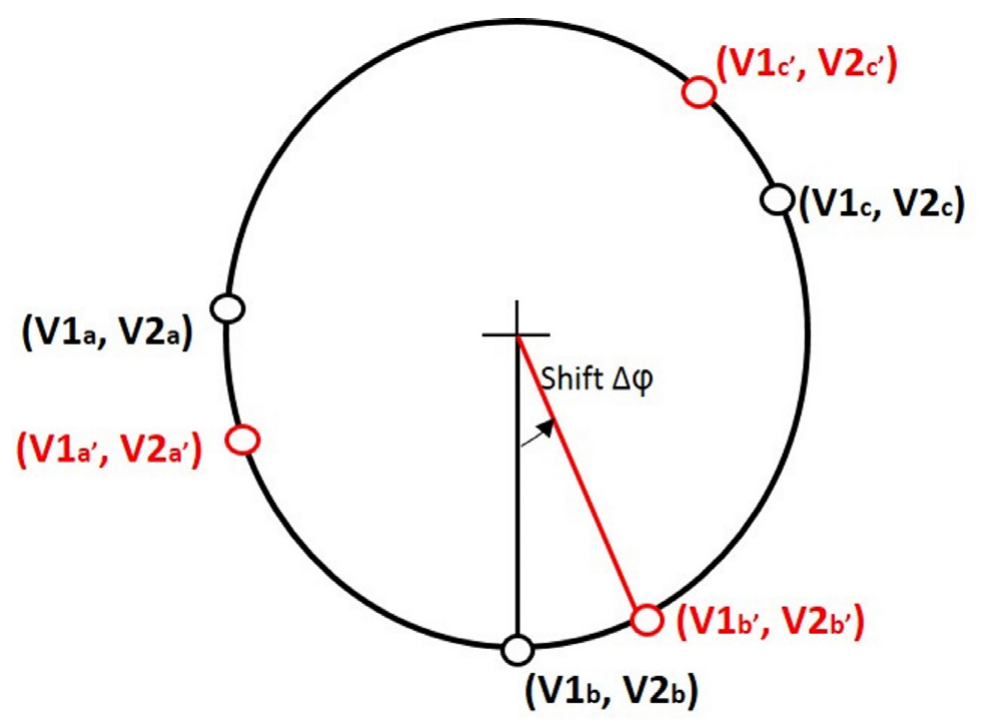

Figure 4.8: Each set of three different output voltages allows to calculate a set of instant values (off1,off2) of the offset voltages. ( $\left.V 1_{a}, V 2_{a} ; V 1_{b}, V 2_{b} ; V 1_{c}, V 2_{c}\right)$ and $\left(V 1_{a^{\prime}}, V 2_{a^{\prime}} ; V 1_{b^{\prime}}, V 2_{b^{\prime}} ; V 1_{c^{\prime}}, V 2_{c^{\prime}}\right)$ represent two sets of sensor outputs. 


$$
\begin{aligned}
& \operatorname{off} 1=\frac{\left(V 1_{b}^{2}+V 2_{b}^{2}-V 1_{a}^{2}-V 2_{a}^{2}\right) *\left(V 2_{c}-V 2_{a}\right)-\left(V 1_{c}^{2}+V 2_{c}^{2}-V 1_{a}^{2}-V 2_{a}^{2}\right) *\left(V 2_{b}-V 2_{a}\right)}{2 *\left(\left(V 2_{c}-V 2_{a}\right) *\left(V 1_{b}-V 1_{a}\right)-\left(V 2_{b}-V 2_{a}\right) *\left(V 1_{c}-V 1_{a}\right)\right)} \\
& \operatorname{off} 2=\frac{\left(V 1_{c}^{2}+V 2_{c}^{2}-V 1_{a}^{2}-V 2_{a}^{2}\right) *\left(V 1_{b}-V 1_{a}\right)-\left(V 1_{b}^{2}+V 2_{b}^{2}-V 1_{a}^{2}-V 2_{a}^{2}\right) *\left(V 1_{c}-V 1_{a}\right)}{2 *\left(\left(V 2_{c}-V 2_{a}\right) *\left(V 1_{b}-V 1_{a}\right)-\left(V 2_{b}-V 2_{a}\right) *\left(V 1_{c}-V 1_{a}\right)\right)}
\end{aligned}
$$

where:

$V 1_{a}, V 1_{b}, V 1_{c}$ represent three output voltages of bridge 1 , without any compensation.

$V 2_{a}, V 2_{b}, V 2_{c}$ are three output voltages of bridge 2 , without any compensation. 
The offset voltages offset 1 and offset2 are calculated from the arithmetic mean of the instant values, off 1 and off2, obtained with different set of sensor outputs during $n$ iterations as shown in Equations (4.26) and (4.27) for the offset voltage of each bridge.

$$
\begin{aligned}
& \text { offset } 1=\sum_{i=1}^{n} \frac{\text { off } 1_{1}+\text { of } f 1_{2}+\ldots o f f 1_{n}}{n} \\
& \text { offset } 2=\sum_{i=1}^{n} \frac{\text { off } 2_{1}+\text { of } f 2_{2}+\ldots o f f 2_{n}}{n}
\end{aligned}
$$

The sensor outputs $V 1$ and $V 2$ are compensated for offset voltage with the factors offset 1 and offset2, obtaining the voltages $V 1$ ov and $V 2 o v$ as presented in Equations (4.28) and (4.29).

$$
\begin{aligned}
& V 1 o v=V 1-\text { offset } 1 \\
& V 2 o v=V 2-\text { offset } 2
\end{aligned}
$$

In addition, it is also possible to calculate the offset voltage per half bridge with Equations (4.30) and (4.31), which are shown with respect to bridge 1 but the same applied for bridge 2. These are derived from Equations (4.19) and (4.21). $V_{R}$ represents the arithmetic mean of the voltage difference between $V_{R 1}$ and $V_{R 3}$ calculated in each iteration in which a set of off 1 and off 2 is calculated.

$$
\begin{aligned}
& \text { of } f p 1=\frac{V_{R}+\text { off } f \text { set } 1}{2} \\
& \text { of } f n 1=\frac{V_{R}-\text { off } f \text { set } 1}{2}
\end{aligned}
$$

Regarding the compensation factors for amplitude imbalance (ai1, ai2), these are derived using sensor outputs with offset voltage compensated (V1ov, V2ov) in which case the Lissajous curve is centred on the origin as shown in Figure 4.6b. This Lissajous curve can be improved by applying factors that allow obtaining a curve closer to a perfect circle; in which the square of the radius is equal to the mean of the values obtained from the sum of the square of V1ov and V2ov at different angles, as shown in Equation (4.32). In theory, this sum should be a fixed value regardless the angle of the magnetic field, however, it shows variations as explained in section 4.2.1.

$$
x^{2}+y^{2}=r^{2}
$$




\subsection{Self-Calibration}

with $x=$ V1ov/ai1; $y=$ V2ov $/$ ai2 $; r^{2}=$ mean $\left((V 1 o v)^{2}+(V 2 o v)^{2}\right)$.

Taking into consideration Equation (4.32), the linear transformation of a circle and a set of voltages at two different angles $\left(V 1 o v_{a}, V 2 o v_{a} ; V 1 o v_{b}, V 2 o v_{b}\right)$, Equations (4.33) and (4.34) have been defined to calculate a set of factors $f 1 a i^{2}$ and $f 2 a i^{2}$, which can be considered as instant values.

$$
\begin{aligned}
& f 1 a i^{2}=\frac{\left(V 1 o v_{b} * V 2 o v_{a}\right)^{2}-\left(V 1 o v_{a} * V 2 o v_{b}\right)^{2}}{\operatorname{mean}(R) *\left(\left(V 2 o v_{a}\right)^{2}-\left(V 2 o v_{b}\right)^{2}\right)} \\
& f 2 a i^{2}=\frac{\left(V 1 o v_{b} * V 2 o v_{a}\right)^{2}-\left(V 1 o v_{a} * V 2 o v_{b}\right)^{2}}{\operatorname{mean}(R) *\left(\left(V 1 o v_{b}\right)^{2}-\left(V 1 o v_{a}\right)^{2}\right)}
\end{aligned}
$$

with

$$
R=(V 1 o v)^{2}+(V 2 o v)^{2}
$$

Then, the compensation factors ai1 and ai2 are determined from the arithmetic mean of the instant values $\left(f 1 a i_{1}^{2}, f 1 a i_{2}^{2} \ldots f 1 a i_{n}^{2}\right)$ and $\left(f 2 a i_{1}^{2}, f 2 a i_{2}^{2} \ldots f 2 a i_{n}^{2}\right)$ obtained during $n$ iterations as presented in Equations (4.35) and (4.36). For each iteration a different set of voltages that belong to one period of the sinusoidal $V 1 \mathrm{ov}$ and $V 2 \mathrm{ov}$ has been used.

$$
\begin{aligned}
& a i 1=\sum_{i=1}^{n} \frac{\sqrt{f 1 a i_{1}}+\sqrt{f 1 a i_{2}}+\ldots \sqrt{f 1 a i_{n}}}{n} \\
& a i 2=\sum_{i=1}^{n} \frac{\sqrt{f 2 a i_{1}}+\sqrt{f 2 a i_{2}}+\ldots \sqrt{f 2 a i_{n}}}{n}
\end{aligned}
$$

Once the compensation factors for offset voltage (offset1, offset2), as well as for amplitude imbalance (ai1, ai2) are calculated, these can be applied in real-time to the sensor outputs (V1,V2) for all angles. Therefore more accurate angle measurements can be obtained from the voltages ( V1ai, V2ai) with both undesired parameters compensated, as shown in Equations (4.37) and (4.38).

$$
\begin{aligned}
& V 1 a i=\frac{V 1 o v}{a i 1} \\
& V 2 a i=\frac{V 2 o v}{a i 2}
\end{aligned}
$$


For self-calibration purposes during the sensor lifetime, Equations (4.24) - (4.27) can be implemented to calculate the offset voltages, as well as Equations (4.28),(4.29), (4.33) - (4.36) to obtain the compensation factors for amplitude imbalance. This will allow keeping the original performance of the sensor over time despite aging effects. The fact that Equations (4.24) and (4.25) share some components, like also occurs between the Equations (4.33) and (4.34) facilitates their implementation. In the following, the verification results obtained by MATLAB as well as FPGA implementation are presented in detail.

\subsubsection{MATLAB implementation}

The proposed compensation method has been verified by a MATLAB implementation using voltages obtained from an analytical model of the sensor, which is included in Appendix B, as well as with voltages measured in commercial AMR sensors KMZ49. Results show that by applying this method, it is possible to reduce the offset voltage, the amplitude imbalance and hence the error in the angle calculation. In the following, the results corresponding to five simulated cases that represent five AMR sensors are discussed in detail.

Table 4.3 presents the offset voltages before and after compensation, in which the offset decreases in both bridges showing values in the order of $\mathrm{mV}$ before and $\mu \mathrm{V}$ after compensation. Taking both bridges into consideration, case 2 shows the largest reduction.

Table 4.3: Offset voltages before and after compensation in the five simulated cases. Each case represents an AMR sensor

\begin{tabular}{|c|c|c|c|c|}
\hline \multirow{2}{*}{ case } & \multicolumn{2}{|c|}{ offset voltage 1} & \multicolumn{2}{c|}{ offset voltage 2} \\
\cline { 2 - 5 } & before & after & before & after \\
\hline 1 & $-0.8 \mathrm{mV}$ & $0.7 \mu \mathrm{V}$ & $2.2 \mathrm{mV}$ & $-1.3 \mu \mathrm{V}$ \\
2 & $-3.4 \mathrm{mV}$ & $3.0 \mu \mathrm{V}$ & $3.9 \mathrm{mV}$ & $-0.6 \mu \mathrm{V}$ \\
3 & $-6.4 \mathrm{mV}$ & $4.0 \mu \mathrm{V}$ & $-1.9 \mathrm{mV}$ & $2.3 \mu \mathrm{V}$ \\
4 & $1.4 \mathrm{mV}$ & $3.8 \mu \mathrm{V}$ & $0.1 \mathrm{mV}$ & $0.2 \mu \mathrm{V}$ \\
5 & $4.8 \mathrm{mV}$ & $-17 \mu \mathrm{V}$ & $-3.8 \mathrm{mV}$ & $-3.8 \mu \mathrm{V}$ \\
\hline
\end{tabular}

Table 4.4 shows the amplitude of the sinusoidal signals in the five simulated cases before and after compensating for amplitude imbalance the bridge outputs that are already compensated for offset voltage. Before compensation, the amplitude differences between the bridge outputs were in the range of $0.1 \mathrm{mV}$ to $2.8 \mathrm{mV}$, showing the largest difference in case 5 . However, after compensation, both bridges in each case show the same amplitude between them. 
Table 4.4: The amplitude of the sinusoidal signals of the bridge outputs with offset voltage compensated and before and after compensating for amplitude imbalance in the five simulated cases. Each case represents an AMR sensor.

\begin{tabular}{|c|c|c|c|c|}
\hline \multirow{2}{*}{ case } & \multicolumn{2}{|c|}{ amplitudes before } & \multicolumn{2}{c|}{ amplitudes after } \\
\cline { 2 - 5 } & bridge1 $(\mathrm{mV})$ & bridge2 $(\mathrm{mV})$ & bridge1 $(\mathrm{mV})$ & bridge2 $(\mathrm{mV})$ \\
\hline 1 & 68.0 & 68.1 & 68.1 & 68.1 \\
2 & 68.7 & 67.8 & 68.2 & 68.2 \\
3 & 68.2 & 68.1 & 68.2 & 68.2 \\
4 & 68.4 & 67.1 & 67.8 & 67.8 \\
5 & 68.9 & 66.1 & 67.5 & 67.5 \\
\hline
\end{tabular}

Table 4.5 shows three types of maximum angle errors that have been calculated in the five simulated cases using the sensor outputs without any compensation (Error1), after compensating for the offset voltage (Error2) and after compensating for offset voltage as well as amplitude imbalance (Error3). In all cases, the maximum errors decrease after compensation, case 2 being the one that shows the largest reduction, if Error1 and Error3 are compared.

Table 4.5: Maximum angle errors obtained in the five simulated cases by applying the proposed compensation method.

\begin{tabular}{|c|c|c|c|}
\hline Case & Error1 (degrees) & Error2 (degrees) & Error3 (degrees) \\
\hline 1 & 1.01 & 0.03 & 0.0017 \\
2 & 2.38 & 0.19 & 0.0029 \\
3 & 2.83 & 0.01 & 0.0051 \\
4 & 0.81 & 0.28 & 0.0038 \\
5 & 3.21 & 0.61 & 0.01 \\
\hline
\end{tabular}

Error1: maximum angle error without any compensation.

Error2: maximum angle error after compensating for offset voltage.

Error3: maximum angle error after compensating for offset voltage and amplitude imbalance.

The proposed compensation method has also been verified by the analysis of the frequency spectrum of the angle error obtained with the calculation of its FFT (Fast Fourier Transform). Table 3.2 in chapter 3 shows that the angle errors exhibit sinusoidal characteristics with different frequencies. The angle error due to offset voltage shows the same frequency as the sensor output, while the error signal due to amplitude imbalance has a frequency twice the sensor frequency. 
Figure 4.9 shows the frequency spectrum with the amplitude of each frequency component in the angle error of case 5, as an example. At the top (A) of the figure, the spectrum of the angle error without any compensation is shown. The largest component is a result of the offset voltage and it appears at $2 \mathrm{kHz}$ which is the frequency of the sensor. In the middle (B), the spectrum after offset voltage compensation is shown with a smaller value at $2 \mathrm{kHz}$ while the largest component is at $4 \mathrm{kHz}$ being related to the error from amplitude imbalance. At the bottom (C), the angle error after compensating for offset voltage and amplitude imbalance is depicted. The frequency components are now smaller than before, and they are mainly present because of additional harmonics included at the sensor outputs.
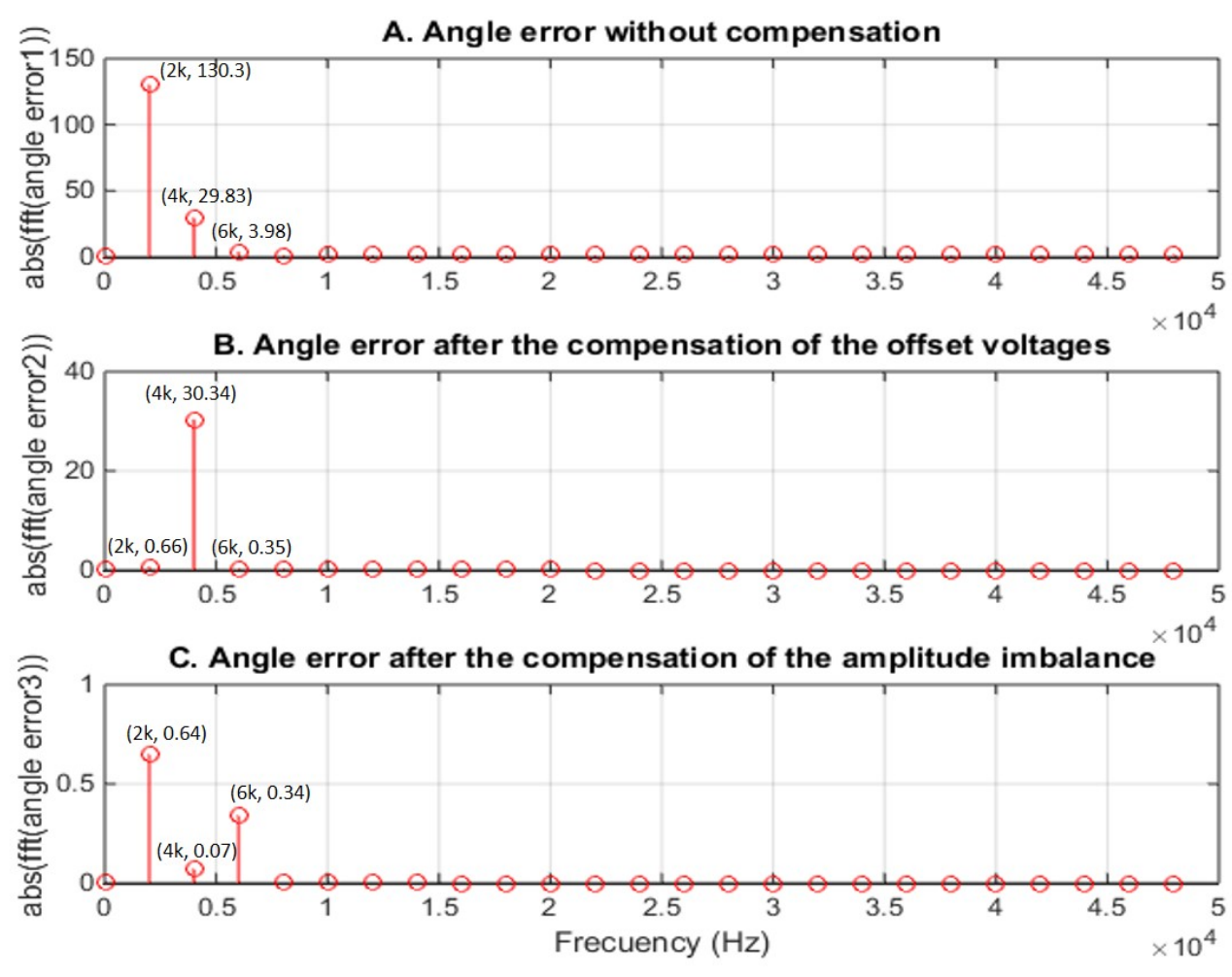

Figure 4.9: Frequency spectrum with the amplitude of the frequency components of the maximum angle error in case 5, which have been obtained by calculating the magnitude of their FFT (Fast Fourier Transform). Notice the different scales.

Figure 4.10 shows the Lissajous curves in case 5, which have been obtained with the sensor outputs without any compensation ( $V 1, V 2)$, after compensating for the offset voltage ( $V 10 v$, V2ov), and after compensating for offset voltage and amplitude imbalance (V1ai, V2ai). Each compensation improves the Lissajous curve that ends close to a perfect circle centred at $(0,0)$, which implies more accuracy in the angle calculation. 


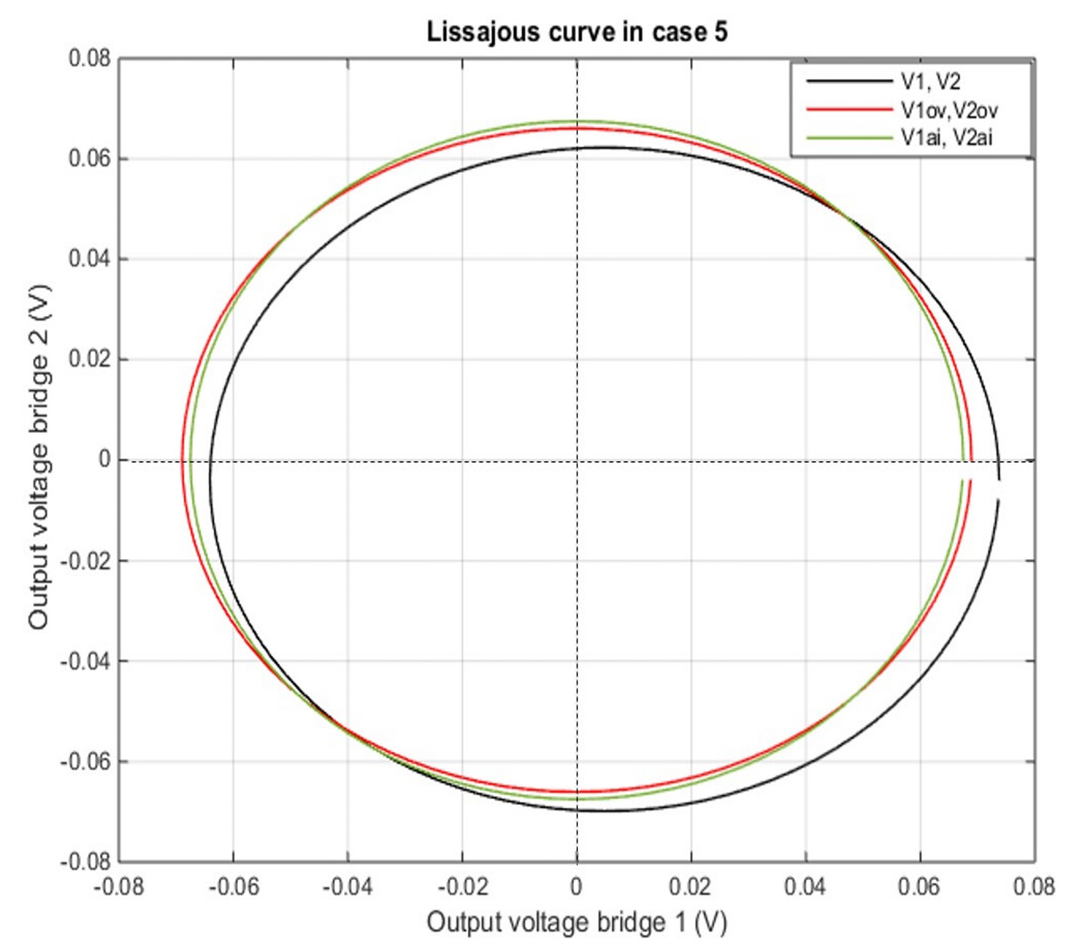

Figure 4.10: Lissajous curves in case 5 obtained with the following sensor outputs: (V1, V2) without any compensation; (V1ov, V2ov) after compensating for offset voltage; (V1ai, V2ai) after compensating for offset voltage and amplitude imbalance.

Regarding the verification of the proposed compensation method using voltages measured in commercial sensors, Table 5.5 presents the maximum angle error in five sensors KMZ49. It includes the errors obtained with the sensor outputs without any compensation (Error1), after compensating for offset voltage (Error2), and after compensating for offset voltage as well as amplitude imbalance (Error3). In all sensors, the maximum angle error after carrying out each compensation has decreased, being the largest reduction in sensor 4 while comparing Error1 and Error3. 
Table 4.6: Variation of the maximum angle error in five commercial AMR sensors after applying the proposed compensation method.

\begin{tabular}{|c|c|c|c|}
\hline Sensor & Error1 (degrees) & Error2 (degrees) & Error3 (degrees) \\
\hline 1 & 0.8635 & 0.1138 & 0.1005 \\
2 & 0.4332 & 0.1326 & 0.1287 \\
3 & 0.4823 & 0.1248 & 0.1129 \\
4 & 0.9867 & 0.1206 & 0.0998 \\
5 & 0.3411 & 0.1353 & 0.1099 \\
\hline
\end{tabular}

Error1: maximum angle error without compensation.

Error2: maximum angle error after compensating for offset voltage.

Error3: maximum angle error after compensating for offset voltage and amplitude imbalance.

The application of the proposed compensation method in all the cases presented above, allowed us to reduce the offset voltages and the amplitude imbalance between the bridge outputs. As a result, the error in the angle calculation, a key parameter in the performance of the sensor, has significantly decreased.

\subsubsection{FPGA implementation}

The proposed compensation method has also been implemented using an FPGA. Next, the two implementations that have been carried out will be discussed in detail.

\section{First implementation:}

In the first implementation, the compensation method for offset voltage and amplitude imbalance has been implemented in VHDL and 16-bit fixed-point arithmetic has been used as much as possible, employing an Altera DE1 board with the following characteristics [Alt12]:

- Cyclon II FPGA.

- Dedicated RAM.

- 18750 logic elements.

- 52 dedicated 9-bit multipliers

- System frequency: $50 \mathrm{MHz}$.

Next, the assumptions made concerning the setup are presented as well as a functional overview of the designed system; finally, the results obtained from the 
implementation are provided.

Assumptions regarding the setup. The proposed compensation method is expected to be implemented in a digital part placed after the A/D conversion of the sensor outputs, as shown in Figure 4.11. However, for our testing purposes, the A/D converter has been replaced by a memory in which the data of the sensor outputs are stored. The maximum sampling frequency of the system is $16 \mathrm{MHz}$ because handling a single sample of inputs takes three cycles of the system running at $50 \mathrm{MHz}$.

Concerning the system inputs, a full period of the sinusoidal signals at the sensor outputs is required to perform the calculations of the compensation factors. The detection of a cycle is executed by finding the zero-crossing of the signals as explained below. It is assumed that at the moment of collecting the data of the signals the magnet generating the magnetic field rotates in the same direction, so a complete cycle of the sinusoidal signals at the sensor outputs can be acquired.

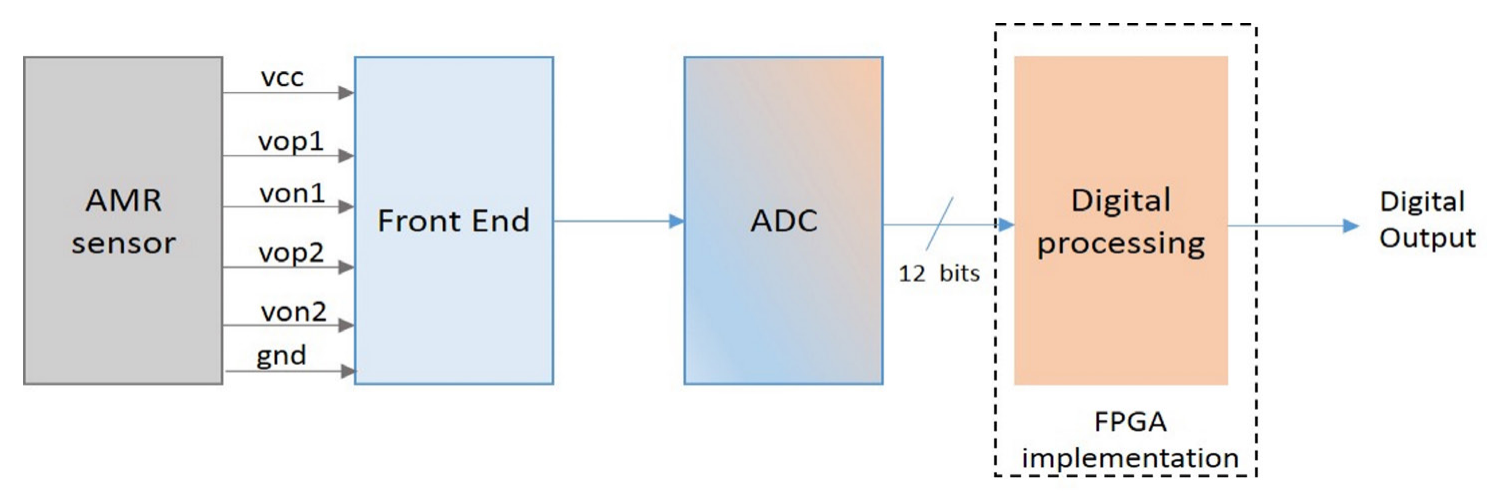

Figure 4.11: Schematic of our AMR sensor system.

Functional overview of the design system. Figure 4.12 depicts an overview of the implemented design in which a time-critical part and a non-time critical part are included. The time-critical part is aimed to compensate the sensor outputs for offset voltage and amplitude imbalance in real-time. This part has dedicated arithmetic units to guaranty they are available if new data needs to be processed.

The non-time critical part calculates the compensation factors used by the timecritical part. For this, first, a full cycle of the sensor outputs is collected and stored in the memory. Then, the compensation factors for offset voltage are calculated using the stored data. After that, the cycle stored in the memory is offset compensated to be used in the calculation of the compensation factors for amplitude imbalance. 


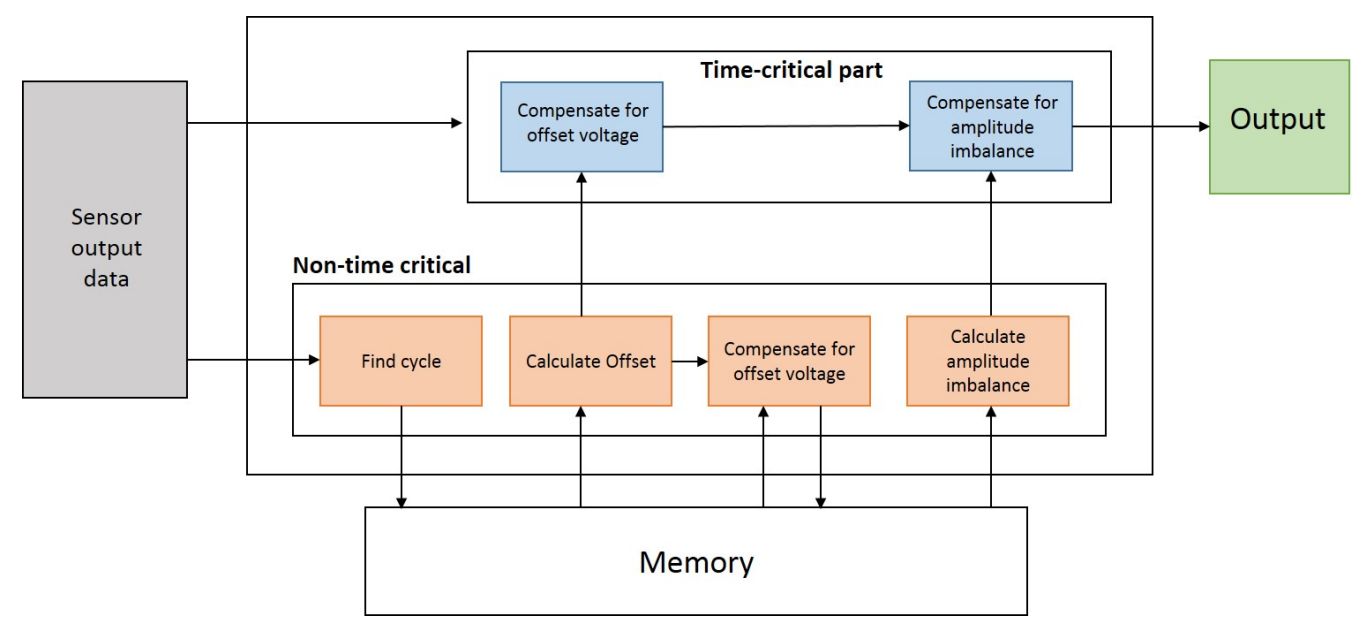

Figure 4.12: Functional overview of the design implemented on FPGA.

The functions described above for the non-critical part are represented as small blocks in the middle of Figure 4.13, in which the architectural overview of this part of the system is shown. Each block refers to a VHDL component, while the block around them represents the main state machine responsible for these blocks to receive the proper inputs and be connected to the arithmetic units at the right time. The VHDL components calculate the instant values of the offset voltage and the amplitude imbalance. The main state machine calculates the arithmetic mean of these values to obtain the compensation factors used afterwards to compensate in real-time the sensor outputs.

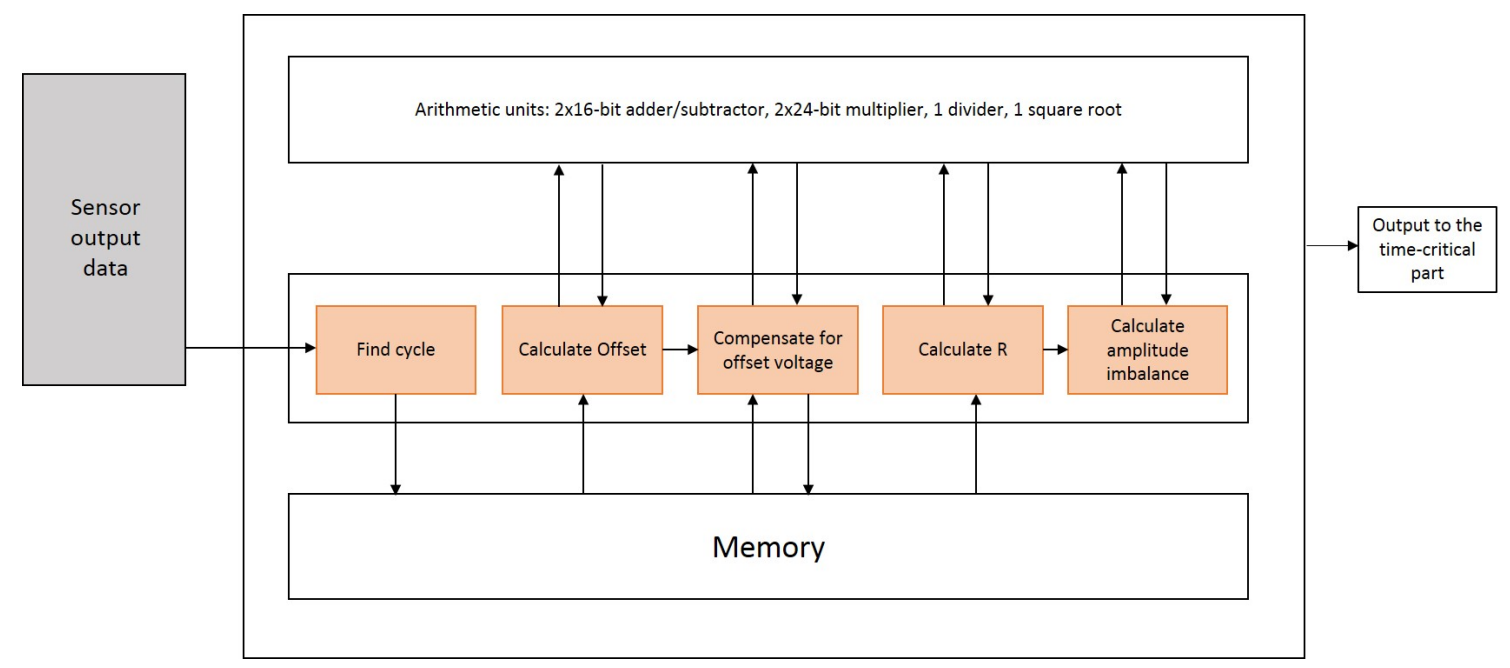

Figure 4.13: Overview of the non-time critical part. 


\subsection{Self-Calibration}

The block termed "Find cycle" is responsible for saving a full cycle of the signals in the memory of the FPGA. A cycle is determined by finding the zero-crossings, which is done by taking into consideration that a cycle starts and stops when the previous sample is negative, and the current sample is positive. The capacity of the memory is limited, and the number of samples in one cycle is not known in advance. Therefore, it is first necessary to determine the percentage of samples that should be stored by counting the number of samples in one cycle to calculate the proportion of samples that can be stored in the next cycle. For example, if a cycle has 400 samples, and the memory has a capability for 128 samples, one in 3 (400/128 rounded down) samples can be stored. This procedure is valid assuming that two consecutive cycles differ no more than $10 \%$ in length. Also, it is considered that the cycles are never longer than 16777215 samples due to computational limitations.

The number of samples of the sinusoidal signals in the sensor outputs that are used for calculating the compensation factors can affect the accuracy of the results. Table 4.7 presents the calculated compensation factors for offset voltage and amplitude imbalance of an AMR sensor considering different amounts of samples of the sensor outputs in a 16-bit architecture with a 32/16 bit divider. Less samples result in less accurate values, as occurs with the factors obtained using 22 samples as compared with the results in the case of 90 samples. These last values can be considered as the expected ones.

Table 4.7: The number of samples of sensor outputs versus the accuracy of the calculated compensation factors of an AMR sensor.

\begin{tabular}{|c|c|c|}
\hline Sensor outputs & offset voltage & Amplitude imbalance \\
\hline 90 samples V1 & 0.000851 & 1.005646 \\
90 samples V2 & -0.000202 & 1.005249 \\
\hline 45 samples V1 & 0.000854 & 1.005856 \\
45 samples V2 & -0.000221 & 1.005351 \\
\hline 22 samples V1 & 0.000949 & 1.023285 \\
22 samples V2 & -0.000263 & 1.020966 \\
\hline
\end{tabular}

The block labelled "Calculate offset" in Figure 4.13 implements the Equations (4.24) and (4.25) to calculate the instant values of the compensation factors for offset voltage (off1, off2). The two blocks defined as "Calculate R" and "Calculate amplitude imbalance" also shown in Figure 4.13 are employed to calculate the instant values of the compensation factors for amplitude imbalance (f1ai, f2ai). However, because of the fact that multiplications demand less resources of the system than divisions, Equations (4.33) and (4.34) have been rewritten to obtain $1 /(f 1 a i)^{2}$ and $1 /(f 2 a i)^{2}$. In this way, the sensor outputs can be multiplied by the compensation factors for amplitude imbalance ai1 and ai2, instead of dividing them as shown in 
Equations (4.37) and (4.38). Each block mentioned above consists of a state machine to perform the calculations step by step connecting the proper arithmetic units, while the main state machine ensures that these components receive the required input values.

Results. The implementation has been accomplished with a VHDL language system description and using 16-bit multipliers, a 24-bit adder/subtractor and a $32 / 16$ bit divider. The system consists of 3600 logical elements including those needed to connect the memory used to store a full cycle of the signals. The number of system cycles required to calculate the compensation factors depends on the number of samples stored in memory. For the 90 samples of sensor outputs used in our case, from which 30 instant values of offset voltage and amplitude imbalance have been calculated, the system needs 6100 cycles, which means 0.00012 seconds at a clock frequency of $50 \mathrm{MHz}$.

Four datasets that represent four AMR sensors have been used to verify the FPGA implementation as presented in Table 4.8. This includes the compensation factors for offset voltage and amplitude imbalance, which have been calculated as follows. First, with a MATLAB implementation (Theoretical) in which floating-point calculation is used by default, and second, with a MATLAB implementation but the results are rounded off (Rounded) after every operation to represent fixed-point calculation and finally, the results obtained from the FPGA implementation. For all cases, the FPGA values are very close to the factors calculated using rounding after every step. The average difference between theoretical and FPGA values are 5.25 $.10^{-6}$ for the offset voltage and $157.10^{-6}$ for amplitude imbalance. The accuracy of the factors for amplitude imbalance can be improved by a better calculation of the required square-root, but also by considering architectures with a larger number of bits. We have verified in MATLAB with variables of 16-bits (implemented in FPGA) and 24-bits, showing that the last results in five times more accurate results for the amplitude imbalance if compared with the theoretical values. However, this also implies more required resources of the system. 
Table 4.8: Comparison of compensation factors calculated by MATLAB implementation using floating-point representation (Theoretical), rounding the results after each operation (Rounded) and with the FPGA implementation. Each dataset represents an AMR sensor.

\begin{tabular}{|l|ccc|ccc|}
\hline \multirow{2}{*}{ Dataset } & \multicolumn{3}{|c|}{ Offset voltage } & \multicolumn{3}{c|}{ Amplitude imbalance } \\
\cline { 2 - 7 } & Theoretical & Rounded & FPGA & Theoretical & Rounded & FPGA \\
\hline Data1,V1 & 0.000853 & 0.000851 & 0.000851 & 1.00586 & 1.00564 & 1.00574 \\
Data1,V2 & -0.000209 & -0.000202 & -0.000202 & 1.00535 & 1.00525 & 1.00537 \\
\hline Data2,V1 & 0.001668 & 0.001659 & 0.001659 & 1.00588 & 1.00568 & 1.00574 \\
Data2,V2 & -0.000609 & -0.000612 & -0.000607 & 1.00524 & 1.00503 & 1.00540 \\
\hline Data3,V1 & -0.001310 & -0.001318 & -0.001316 & 1.00580 & 1.00558 & 1.00568 \\
Data3,V2 & -0.001483 & -0.001486 & -0.001480 & 1.00527 & 1.00513 & 1.00534 \\
\hline Data4,V1 & 0.014869 & 0.014868 & 0.014862 & 1.00803 & 1.00775 & 1.00793 \\
Data4,V2 & 0.007208 & 0.007206 & 0.007213 & 1.00336 & 1.00330 & 1.00388 \\
\hline
\end{tabular}

In this section, the proposed compensation method for offset voltage and amplitude imbalance has been validated through hardware implementation and using the fixed-point representation. Next, the results obtained by implementing the compensation method for offset voltage using floating-point representation and a soft-core processor is presented.

\section{Second implementation}

For this implementation the FPGA Altera EP4CE22F17C6 has been used, which is included in the DE0-nano development board with the following characteristics [Ter99]:

- $22 \mathrm{k}$ logic elements.

- the NIOS II CPU.

- 64KB on-chip memory .

- 32MB external DRAM .

As in the first implementation, it is also considered that data from a period of the sinusoidal signals at the sensor outputs is already acquired. The Equations (4.24) up to and including (4.27) have been implemented using the IEEE 754 floating-point standard. Figure 4.14 shows the architecture of the system, in which the NIOS II CPU in the FPGA is a 32-bit fixed-point processor with no hardware for floating-point number operation, so the floating-point computation is executed by software resulting in a longer processing time as compared to a hardware floating-point implementation. 
In this case, a hardware divider module and a barrel shifter have been added to increase the speed of the divide/floating point process. The system software is stored in the DRAM, while the JTAG port is used to program the device as well as to read the computational results from an external computer.

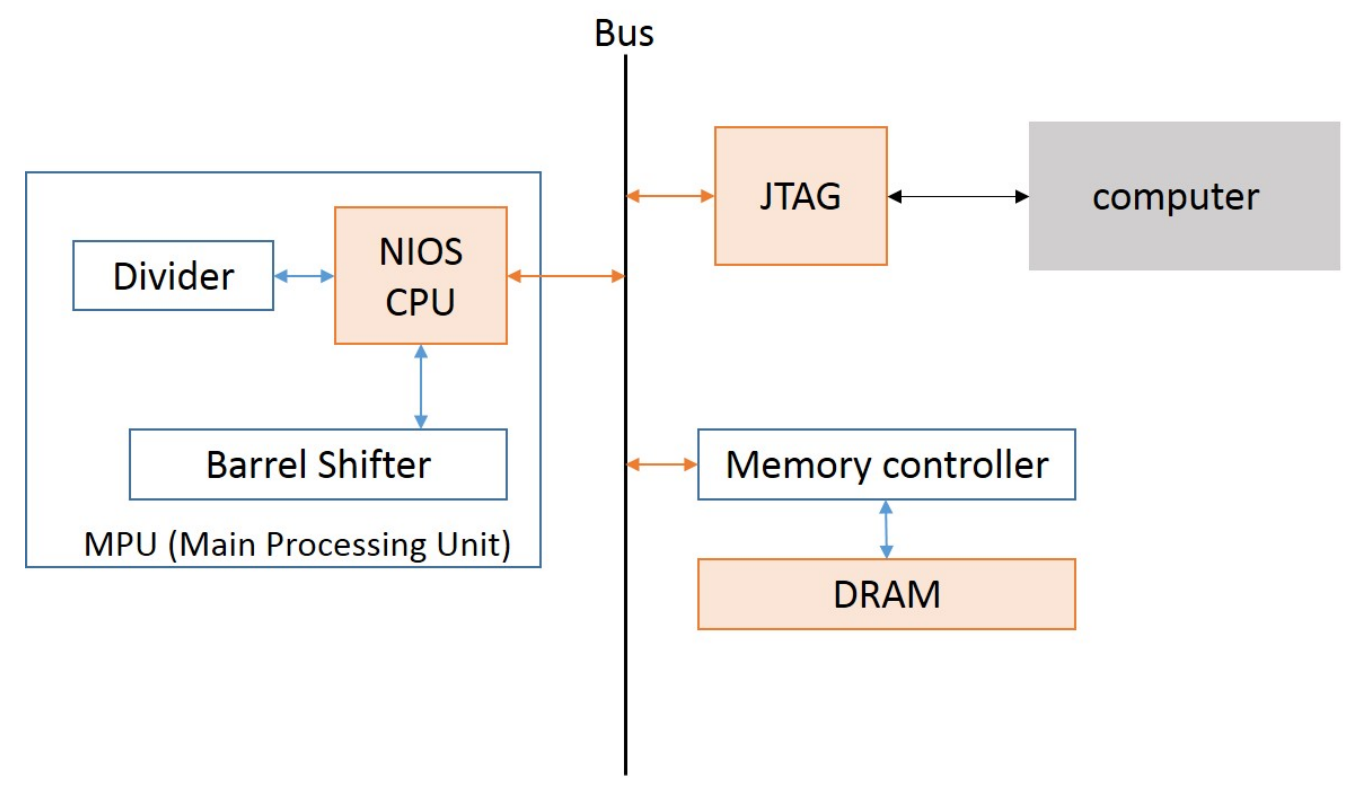

Figure 4.14: Architecture of the implemented FPGA system.

At the hardware level, the system consists of a clock module, the NIOS II CPU, a math module that includes multiplier, divider and barrel shifter, and a DRAM controller module. With regard to the software, a serial sequence to calculate the compensation factors has been implemented, as shown in the flowchart of the main process loop in Figure 4.15. First, the input data indicated by the data index that consists of three data pointers for the three sensor outputs required in each iteration are read to compute the instant values of the offset voltages (off1, off2) employing Equations (4.24) and (4.25). These values are added into accumulation variables to compute afterwards their arithmetic mean obtaining the offset voltages (offset1, offset2), as provided in Equations (4.26) and (4.27). 


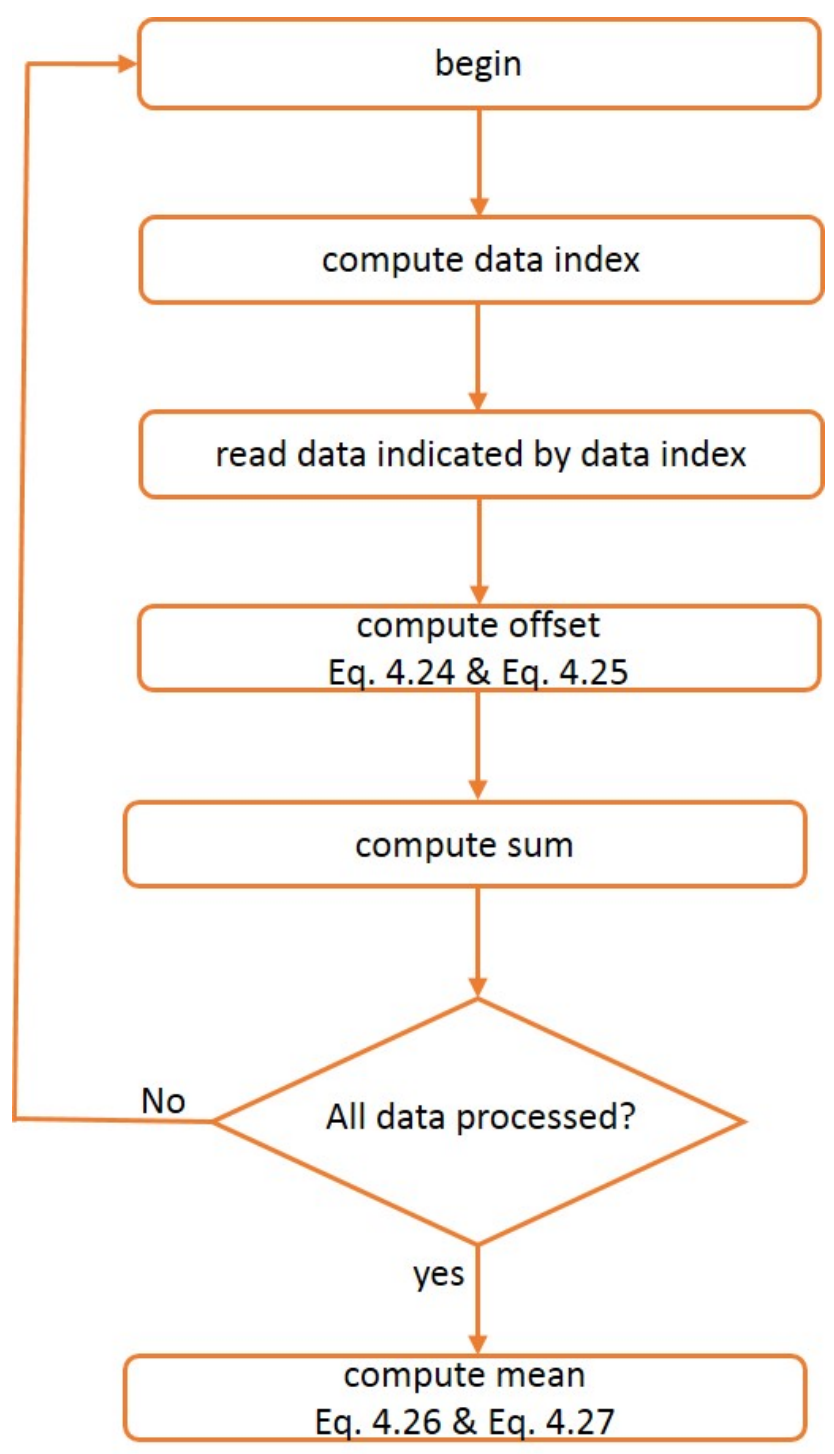

Figure 4.15: Chart of the main process flow for calculating the offset voltage.

Using a clock frequency of $50 \mathrm{MHz}, 100$ instant values of the offset voltages have been computed for a dataset with 100 samples of sensor outputs. Figure 4.16 shows the results obtained in which the computational results for (mean_off1) and (mean_off2) are very close to the reference values of $-0.5786 .10^{-3} \mathrm{~V}$ for offset1 and $3.146 \cdot 10^{-3} \mathrm{~V}$ for offset2, which have been calculated in MATLAB. The average difference between the reference and the computational values is $2.5 \mu \mathrm{V}$, which is equal to half the average error $(5.25 \mu \mathrm{V})$ obtained in the first implementation for the calculation of the offset voltages. Although the calculation of the compensation factor for amplitude imbalance was not implemented, more accurate results can be expected compared with the first implementation with 16-bit architecture, as floating-point arithmetic can be used. In principle, floating-point calculation provides better performance than fixed-point calculation, if operations such as multiplication, 
division, square-root calculation are required. The execution of the floating-point computation by software implies a longer processing time, so it is recommended to perform the hardware floating-point implementation.

\begin{tabular}{|c|c|c|}
\hline & \multicolumn{2}{|c|}{ O Bre... Gor Exp... ${ }_{01010}^{1010}$ Regi... O Me... (x)=Vari... \&3 范 } \\
\hline & & 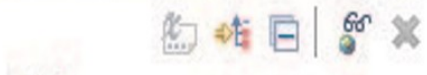 \\
\hline \multirow{4}{*}{ Sensor outputs } & Name & Value \\
\hline & $D 8 \mathrm{v} 1$ & $0 \times 03 f f f l_{a} 4$ \\
\hline & $D \theta \mathrm{v} 2$ & $0 \times 03 \mathrm{fffb} 34$ \\
\hline & $(x)=i \_x 1$ & 99 \\
\hline \multirow[t]{3}{*}{ Data pointers } & $(x)=i_{-} x 2$ & 32 \\
\hline & $(x)=i_{-} x 3$ & 65 \\
\hline & $(x)=i$ & 101 \\
\hline \multirow{2}{*}{$\begin{array}{l}\text { Factors calculated to be } \\
\text { used in the equations }\end{array}$} & $(x)=A$ & $5.4743653 \mathrm{E}-4$ \\
\hline & $(x)=B$ & $-1.780259 \mathrm{E}-4$ \\
\hline \multirow{4}{*}{ Instant offsets } & $D$ off1 & $0 \times 03 \mathrm{fffec} 4$ \\
\hline & $\triangleright \theta$ off2 & $0 \times 03 \mathrm{fffe} 54$ \\
\hline & $(x)=$ sum_off1 & -0.057479966 \\
\hline & $(x)=$ sum_off2 & 0.314848 \\
\hline \multirow{2}{*}{ Offset voltages } & $(x)=$ mean_off1 & $-5.7479966 \mathrm{E}-4$ \\
\hline & $(x)=$ mean_off2 & 0.00314848 \\
\hline
\end{tabular}

Figure 4.16: Screenshot of the offset results of an AMR sensor with our second FPGA implementation.

The functionality of the proposed compensation method for offset voltage and amplitude imbalance has been verified in MATLAB, and by FPGA implementation using a 16-bit architecture with fixed-point representation. Additionally, the calculation of the compensation factors for offset voltage has also been implemented using the IEEE 754 floating-point standard with 32 bits, which shows more accurate results than the fixed-point representation. However, an accuracy improvement can be achieved in fixed-point operation using an architecture with a larger number of bits, for instance, 24 or 32 bits and optimising the algorithm used to perform the calculation of the square-root. Although the feasibility of implementing fixed-point or floating-point operation also depends on the characteristics of the system used, in general, a fixed-point operation demands less processing resources and provides faster results. 


\subsection{Conclusions}

\subsection{Conclusions}

In this chapter, the incorporation of self-X properties in AMR sensors used for angle measurements is proposed. The main goal is to maintain the sensor performance over time by compensating for aging effects. The self-monitoring feature allows keeping track of the error in the angle calculation, a key parameter in the performance of the sensor, while with self-calibration it is possible to update the compensation factors online during the sensor lifetime.

A set of equations has been proposed to perform self-monitoring by calculating the maximum angle errors due to the undesirable parameters included in the sensor outputs. These are based on the sinusoidal characteristics of the bridge outputs and the Pythagorean identity. From this identity it is implied that the addition of the square of the sensor outputs should be a fixed value no matter the magnetic angle; otherwise the resulting variation is determined by the largest source of angle error that is present. The offset voltage produces the largest error in sensor outputs without any compensation; in the second place comes the amplitude imbalance for sensor outputs with offset voltage compensated, and as last the harmonics in the sensor outputs after compensating for the other two error sources.

The proposed equations have been validated using data from an analytical model of the sensor, but also from data of commercial sensors. The results show they provide an accurate estimation of the angle errors. In the proposed simplified version, less processing resources are demanded from the system; hence these equations are more suitable to be used online during the sensor's lifetime. The offset voltage is the most significant source of angle error, so for practical purposes, it is possible to focus on this angle error only and verify the other two sources of angle errors if a more in-depth analysis of the performance of the sensor is required. So far, online monitoring of the sensor performance is usually not applied. However, this will change in the future as a reduction of the tolerance band for drifting of the performance of the sensor is expected.

Regarding the self-calibration feature, it allows updating online the compensation factors for offset voltage and amplitude imbalance. These are the first and second source of angle error at the start of the sensor life, but they also show the first and second largest drift during the sensors' lifetime. The compensation method is based on the geometrical characteristics of the Lissajous curve obtained from the sensor outputs and it has been verified by MATLAB calculations, but also with an FPGA implementation using data from an analytical model of the sensor and from data of commercial sensors. The results show that by the usage of this method the offset voltage could be reduced, as well as the amplitude imbalance between the sensor outputs and hence the resulting error in the angle calculation during the sensor lifetime. Compared to other methods already proposed, our approach does not require any particular setup, so it can be used on-site. Although the compensation factors cannot be calculated in real-time, it is actually not necessary because the undesired parameters such as the offset voltage drift slowly over time. Therefore, as 
processing resources are limited during the sensors' lifetime, it is better to compensate the sensor outputs in real-time but calculate the compensation factors only when it is actually required as determined by the self-monitoring feature.

Until now, compensation factors for AMR sensors are usually calculated during a calibration process under factory conditions just before shipping. The accuracy demanded from the sensor allows having an additional error during the sensor lifetime due to aging effects. However, the future trend clearly indicates it will be necessary to carry out more accurate angle measurements. This is especially true in safety-critical applications, which implies it will be required to maintain the sensor performance over a long period of time. The addition of self-X properties as we proposed will allow the sensor to handle performance degradation under its operating conditions during its lifetime. 


\section{References}

[Alt12] Altera. "DE1 Development and education board. User manual". 2012.

[App16] Appalachian state university. "http://physics.appstate.edu", 2016. Accessed 2017-03-10.

[Bra17] B. Brad. "http://www2.ohlone.edu/people2/bbradshaw/handouts/". 2017. Accessed 2017-03-20.

[Die00] K. Dietmayer and M. Weser. "Contactless Angle Measurement using KMZ41 and UZZ9000". Application Note. AN00023, Philips Semiconductors, Germany, 2000.

[Isl10] M. Isler, B. Christoffer, and G. Schoer. "Optimisation of surface passivation for highly reliable angular AMR sensors". Physica Status Solidi (c), volume 7(2), pp. 436-439, 2010.

[Joh11a] M. A. Johar, R. Freier, and A. Koenig. "Adding self-x capabilities to AMR sensors as a first step towards dependable embedded systems". In Proceedings of the Ninth Workshop on Intelligent Solutions in Embedded Systems (WISES), pp. 41-46, July 2011.

[Joh11b] M. A. Johar and A. Koenig. "Case Study of an Intelligent AMR Sensor System with Self-x Properties". Soft Computing in Industrial Applications, pp. 337-346, 2011.

[Lar14] J. Lara and A. Chandra. "Position error compensation in quadrature analog magnetic encoders through an iterative optimization algorithm". In 40th Annual Conference of the IEEE Industrial Electronics Society, pp. 3043-3048, Oct 2014.

[Liu00] J. Liu and U. Fruhauf. "Design for self-calibration of instrumentation". In world congress of international measurement confederation XVI IMEKO, Sep 2000.

[Ter99] Terasic. "http://www.terasic.com.tw", 1999. Accessed 2017-03-20.

[Von06] V. Von Tils. "Trends and Challenges in Automotive Electronics". In IEEE International Symposium on Power Semiconductor Devices and IC's, pp. $1-3$, June 2006.

[Zam15] A. Zambrano and H. G. Kerkhoff. "Online digital offset voltage compensation method for AMR sensors". In Proceeding of IEEE International Instrumentation and Measurement Technology Conference (I2MTC), pp. 1512-1515, May 2015.

[Zam16a] A. Zambrano and H. G. Kerkhoff. "Online digital compensation Method for AMR sensors". In proceeding of IFIP/IEEE International Conference on Very Large Scale Integration (VLSI-SoC), pp. 1-6, Sept 2016. 
[Zam16b] A. Zambrano and H. G. Kerkhoff. "Online monitoring of the maximum angle error in AMR sensors". In proceeding of IEEE 22nd International Symposium on On-Line Testing and Robust System Design (IOLTS), pp. 211-212, July 2016. 


\title{
Chapter 5
}

\section{Fault-tolerance in AMR sensors}

\begin{abstract}
Among the dependability requirements for automotive applications presented in chapter 2 is included that electronic systems should be capable of continuing operating despite an unexpected failure; this is especially important in safety-critical applications like in automotive. This chapter is focussed on proposing a fault-tolerant system aimed to allow AMR sensors for angle measurements to continue operating even if certain catastrophic faults occur. In the following, the effects of catastrophic faults in AMR sensors are presented. Then, our proposed fault-tolerant system that performs fault-detection, fault-location, fault-reconfiguration and fault-recovery is explained in detail.
\end{abstract}

\subsection{Introduction}

The automotive industry is moving ahead to introduce more X-by-wire systems which means that the mechanical and hydraulic systems traditionally used are being replaced by fully electronic systems. Although this will allow enhancing the vehicle driving performance as well as safety, electronic components show lower reliability and different fault behaviours as compared to mechanical parts [Ise02]. An electronic component can fail suddenly and without warning. Hence, the incorporation of fault-tolerant techniques to avoid the system to suddenly stop working should be considered; especially in safety-critical applications in which a fault can represent a threat for people or the environment [Buj04], [Tre01]. After a fault occurs in a vehicle, the driver should be alerted about the faulty condition and advised to get it repaired, but the basic vehicle operations must be maintained for a limited time period [Sin11], [Ise02]. In case it is required, it is even possible that the car enters into a partial operating mode in which reduced functionality is permissible, as long as there is no risk in terms of safety.

Part of this chapter has been presented at the 21nd IEEE International Symposium on On-line Testing and Robust System Design (IOLTS) [Zam15] 
A fault can be defined as any abnormal condition that can lead to an error and to a subsequent system failure, which means the system cannot deliver its intended function [Abd06]. In analog electronics, a fault is usually classified as catastrophic or parametric. A catastrophic fault also defined as hard fault refers to all those changes that make the circuit to stop working. Among these faults are short or open ones as well as extreme out-of-range parameters that can lead to a completely malfunctioning circuit. In the case of a parametric fault, the system continues its operation, but one or more parameters are out of specifications mostly due to performance degradation [Kab05], [Lay08].

A sensor, like any other analog electronic component, can be affected by parametric and/or catastrophic faults. In the case of AMR sensors for angle measurements, the addition of self-X properties has been proposed in chapter 4 to prevent that performance degradation leads to parametric faults. Catastrophic faults can be caused by faulty bridge resistances with a short or open condition, loss of the magnet that generates the magnetic field which angle is to be measured or broken sensor connections with regard to the power supply or ground [Die00].

In automotive applications, the sensors should be fail-operational (FO) meaning that one failure is tolerated, so the system continues in operation. Therefore faulttolerant techniques are usually included to handle a fault in such a way that it does not lead to a system failure [Ise02]. Fault-tolerance can be implemented by physical redundancy with one or more extra hardware besides the original element or by analytical redundancy, in which the missing information is obtained from other hardware that is already available or another internal value of the system [Abd06], [Gao15]. Another option is temporal redundancy that focusses on performing the same task several times using the same resources, in case of a failure [Run12].

Most of the fault-tolerant methods proposed so far for AMR sensors are based on physical redundancy, such as the systems proposed by Dietmayer [Die00], Dilge [Dil03] and Infineon [Inf14], which are included in chapter 2. However, in order to satisfy the dependability requirements of automotive applications also presented in chapter 2 , it is important to have fault-tolerant systems that allow providing the required dependability without significantly increasing the cost of hardware [Ise02]. Physical redundancy implies a higher production cost and can be restricted by space and/or weight limitations in the system [Gao15]. Analytical redundancy can be implemented by using other hardware already included in the system or by software, so it poses few restrictions on the production cost and system design [Lay08].

The research presented in this chapter is focussed on proposing a fault-tolerant system based on analytical redundancy to handle catastrophic faults in the bridge resistances of AMR sensors for angle measurements. In addition, it is able to handle a broken connection of the sensor to the power supply or ground by physical redundancy. Once the system detects a fault condition, it determines its location and then applies the required reconfiguration to accomplish that the sensor continues its operation. 
The rest of this chapter is organised as follows. In section 5.2 the catastrophic faults that can occur in AMR sensors and their effect on the angle measurement are presented. Section 5.3 explains the proposed fault-tolerant system and in section 5.4 conclusions are provided.

\subsection{Catastrophic faults in AMR sensors}

The AMR sensors for angle measurements consist of eight magnetoresistances configured into two Wheatstone bridges placed at $45^{\circ}$ with respect to each other. Each bridge, in turn, is made of two half-bridges with the output pins vop1, von1 in bridge 1 and vop2, von2 in bridge 2, as shown the schematic of an AMR sensor in Figure 5.1.
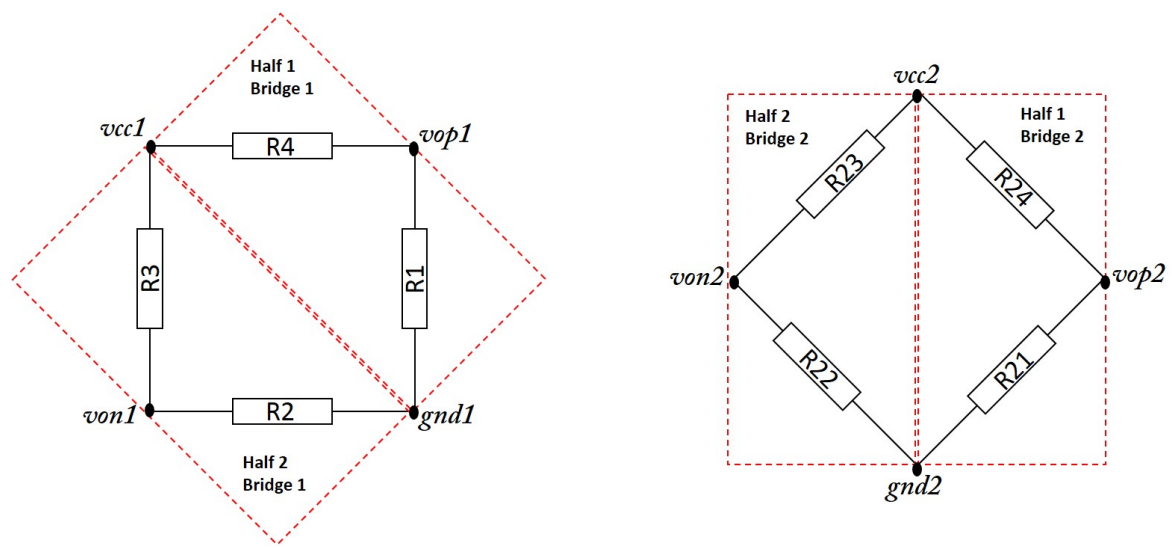

Figure 5.1: Schematic of the Wheatstone bridges of an AMR sensor for angle measurements. The pins vop1, von1, vop2, von2 represent the outputs of the half bridges; $v c c 1, v c c 2$ are connection pins to the power supply and gnd1, gnd2 are connected to ground.

In the saturation state of the sensor, each half-bridge shows ideally an output voltage that consists of a DC value that depends on the power supply plus a sinusoidal signal caused by the magnetoresistance effect; this depends on the angle to be measured as shown in Equations (5.1) and (5.2). Figures 5.2 and 5.3 present the output voltages of the half-bridges in bridge 1 and 2 of an AMR sensor. A value of $5 \mathrm{~V}$ for the power supplies (vcc1, vcc2) are provided and $68 \mathrm{mV}$ for the amplitude of the cosine signals and sine signals resulting from the magnetoresistance effect (amr1,amr2).

$$
\begin{array}{ll}
\text { vop } 1=\frac{v c c 1}{2}+\frac{\operatorname{amr} 1 \cdot \cos (2 \theta)}{2} & \text { von } 1=\frac{v c c 1}{2}-\frac{\operatorname{amr} 1 \cdot \cos (2 \theta)}{2} \\
\text { vop } 2=\frac{v c c 2}{2}+\frac{\operatorname{amr} 2 \cdot \sin (2 \theta)}{2} & \text { von } 2=\frac{v c c 2}{2}-\frac{\operatorname{amr} 2 \cdot \sin (2 \theta)}{2}
\end{array}
$$


The magnetic angle $\theta$ is calculated from the differential outputs of the bridges. In these signals, only the values resulting from the magnetoresistance effect are included, as presented in Equation (5.3).

$$
2 \theta=\arctan \left(\frac{V 2}{V 1}\right)=\arctan \left(\frac{v o p 2-v o n 2}{v o p 1-v o n 1}\right)=\arctan \left(\frac{a m r 2 \cdot \sin (2 \theta)}{\operatorname{amr} 1 \cdot \cos (2 \theta)}\right)
$$

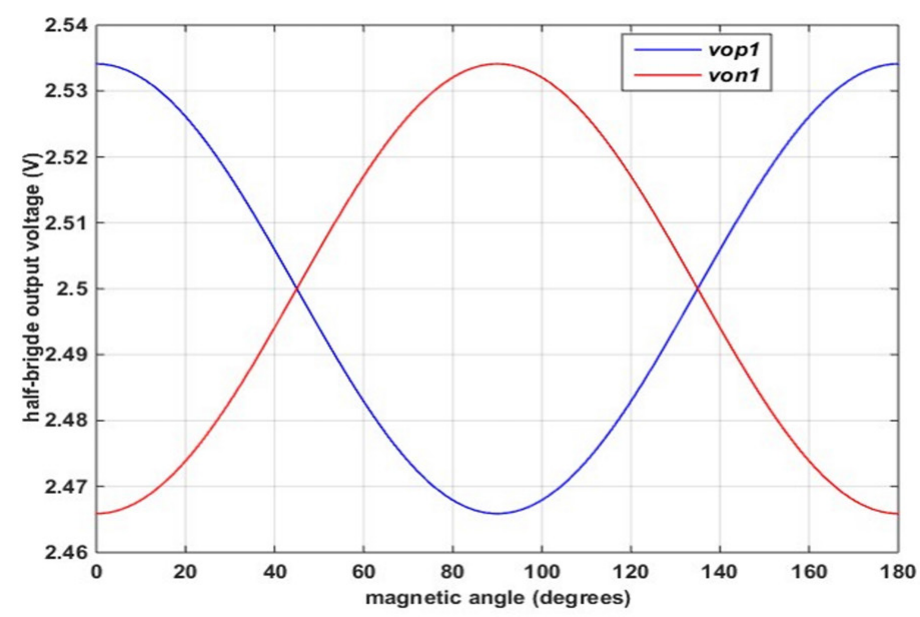

Figure 5.2: Output voltage of half-bridge 1 (vop1) and half-bridge 2 (von1) in bridge 1 of an AMR sensor.

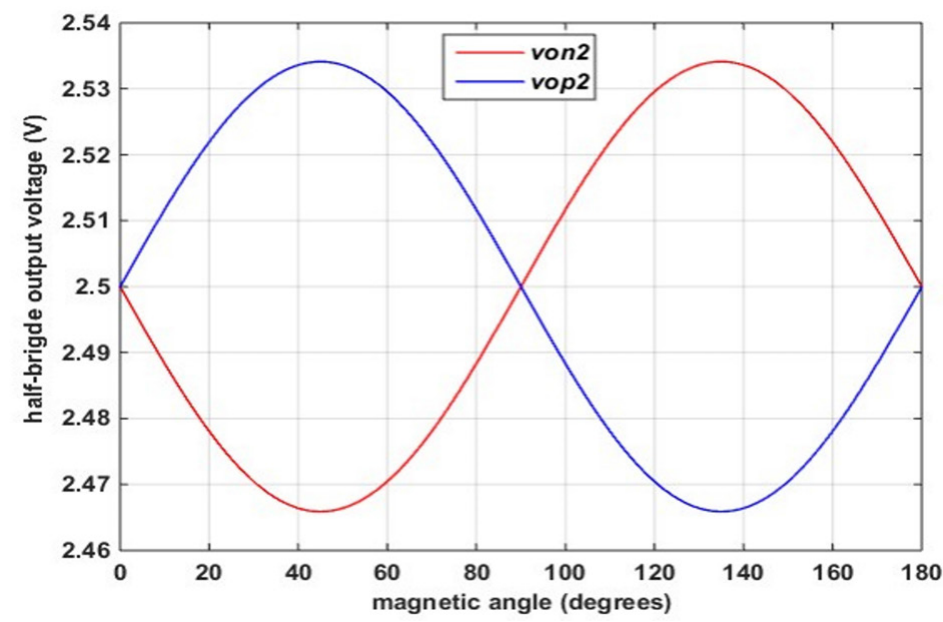

Figure 5.3: Output voltage of half-bridge 1 (vop2) and half-bridge 2 (von2) in bridge 2 of an AMR sensor. 
As the angle is calculated from the sinusoidal signals of the bridge outputs, if a catastrophic fault occurs and one or both signals are lost, then the angle cannot be calculated anymore. This could be caused by faulty bridge resistances with open or short conditions, broken bridge connections to the power supply or ground, but also due to the loss of the magnetic field which angle is to be measured. In the following, the effects of catastrophic faults in AMR sensors with regard to the angle calculation are presented.

\subsubsection{The effect of catastrophic faults in AMR sensors}

In a fault-free sensor, the magnetisation vector of the sensor should follow the magnetic field. Therefore as shown in Figure 5.4, a linear relationship should be obtained if the set magnetic angles are compared with the calculated angles. The sign change of the slope at a set magnetic angle of $90^{\circ}$ is resulting from the characteristics of the sinusoidal signals in the sensor outputs. In addition the detected angle of the sensor is twice the angle of the magnetic field because of the sensor configuration.

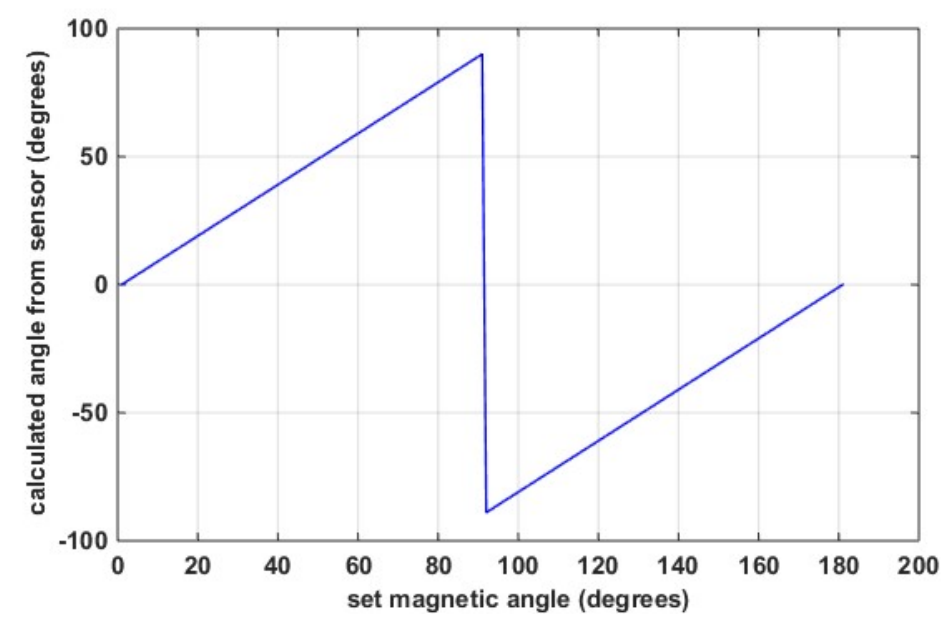

Figure 5.4: Calculated angle with a fault-free sensor versus the set magnetic angle.

However, the linear relationship that is shown in Figure 5.4 can be lost due to open or short conditions in the bridge resistances of the sensor. The effect on the angle calculation depends on the location of the faulty resistance or faulty resistances in the Wheatstone bridges as detailed in the following four cases specified based on the bridge resistances presented in Figure 5.1.

Case one: this refers to an open condition in the resistance R1 or/and R3, or/and a short condition in the resistance $\mathrm{R} 2$ or/and $\mathrm{R} 4$ of bridge 1 . Then, instead of obtaining a calculated angle between $\pm 90^{\circ}$ as shown in Figure 5.4, the calculated angle is between $\pm 1^{\circ}$ irrespective of the set magnetic angle as presented in Figure 5.5. 


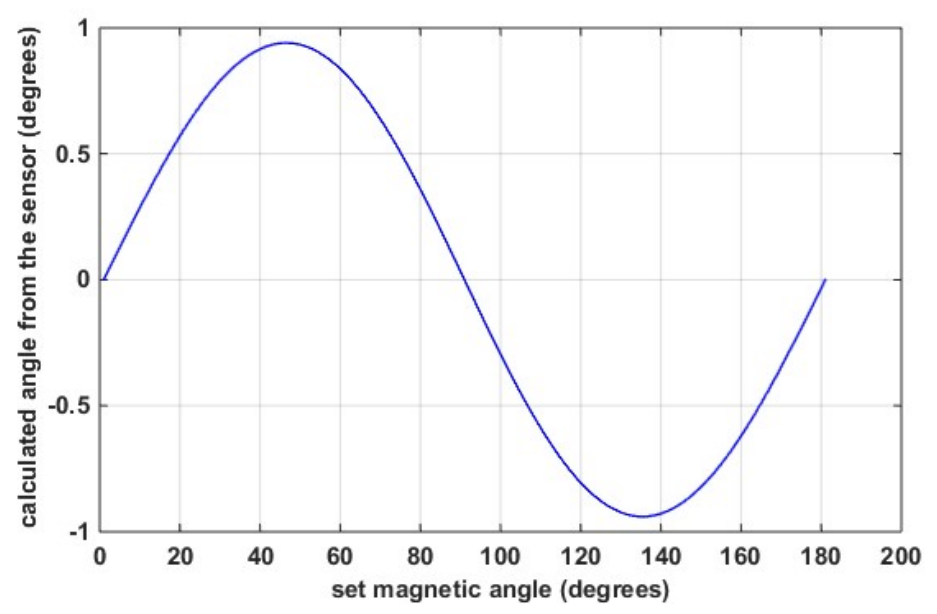

Figure 5.5: Calculated angle from the sensor outputs presented in Equation (5.3) in case one.

Case two: this represents a short condition in the resistance R1 or/and R3, or/and an open in the resistance $\mathrm{R} 2$ or/and $\mathrm{R} 4$. As a result, the calculated angle oscillates between $\pm 90^{\circ}$ depending on the sign of the sinusoidal signal in bridge 2 , as depicted in Figure 5.6.

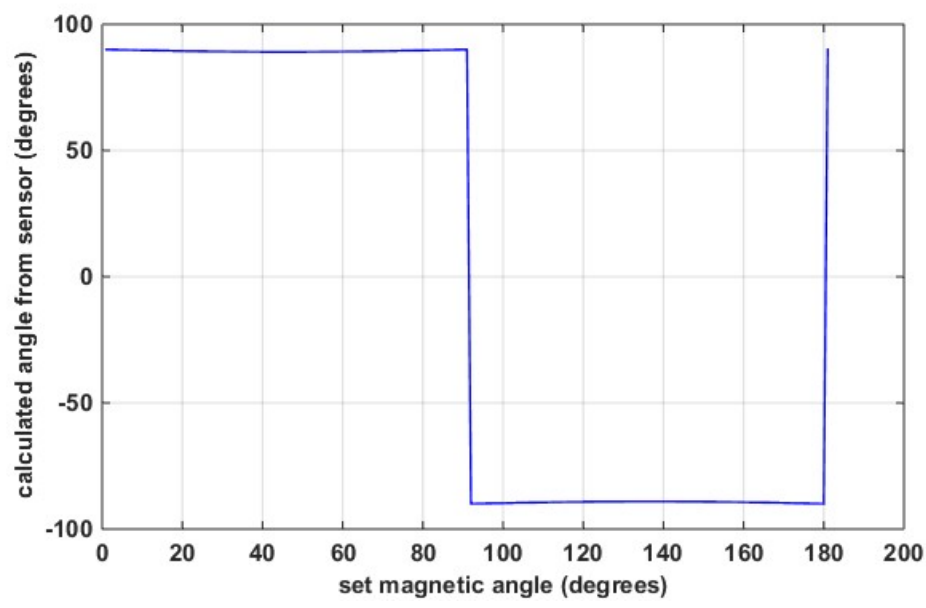

Figure 5.6: Calculated angle from the sensor outputs presented in Equation (5.3) in case two.

Case three: here the faulty resistance is located in bridge 2 with either a short condition in R21 or/and R23, or/and an open condition in R22 or/and R24. This causes that the calculated angle does not follow the set magnetic angle, but shows values close to $-45^{\circ}$, as presented in Figure 5.7. 


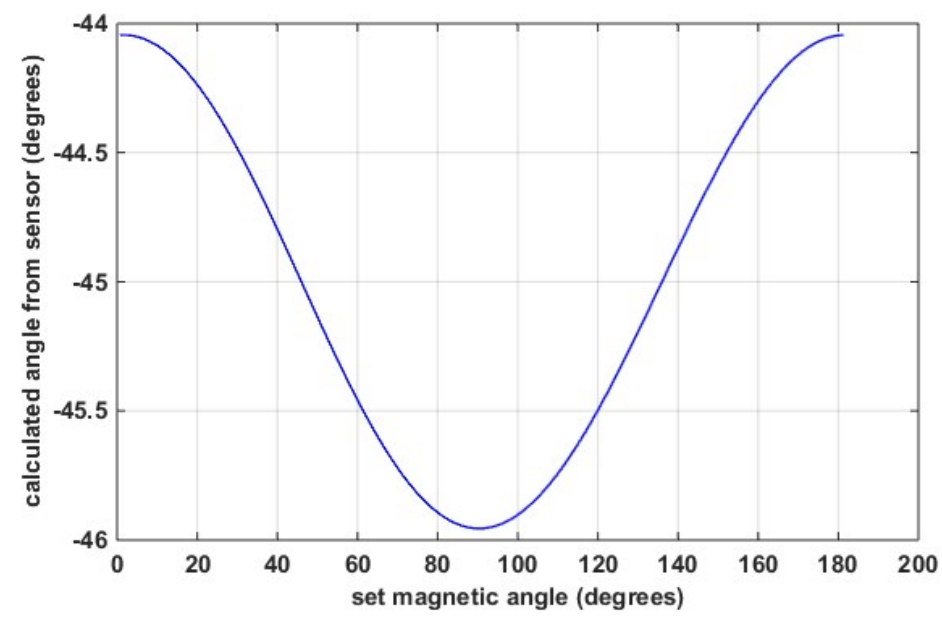

Figure 5.7: Calculated angle from the sensor outputs presented in Equation (5.3) in case three.

Case four: this focusses on an open condition in the resistance R21 or/and $\mathrm{R} 23$, or/and a short condition in the resistance R22 or/and R24. As a result, the calculated angle is always close to $45^{\circ}$ as presented in Figure 5.8.

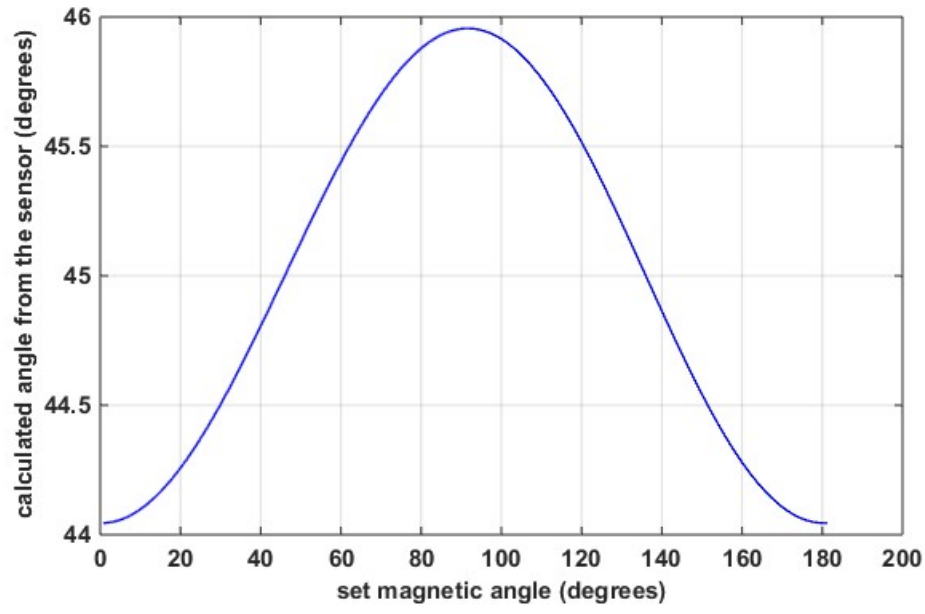

Figure 5.8: Calculated angle from the sensor outputs presented in Equation (5.3) in case four.

As mentioned above, another reason for losing the trigonometric relationship of the bridge outputs is a broken bridge connection to the supply voltage or ground. If this is the case for bridge 1 , then the calculated angle oscillates between $\pm 45^{\circ}$ depending on the sign of the sinusoidal signal in bridge 2 as depicted in Figure 5.9. On the other hand, if it occurs in bridge 2, Figure 5.10 shows that the calculated angle fluctuates between $0^{\circ}$ and $90^{\circ}$ based on the sign on the sinusoidal signal in bridge 1 . 


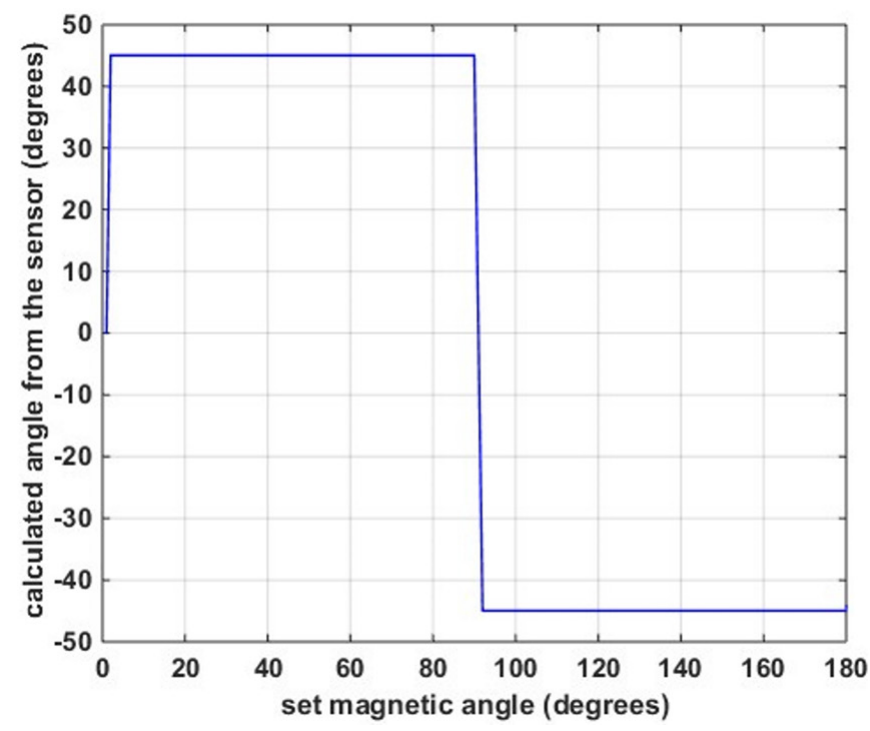

Figure 5.9: Calculated angle from the sensor outputs in case of a broken connection to the power supply or ground in bridge 1 .

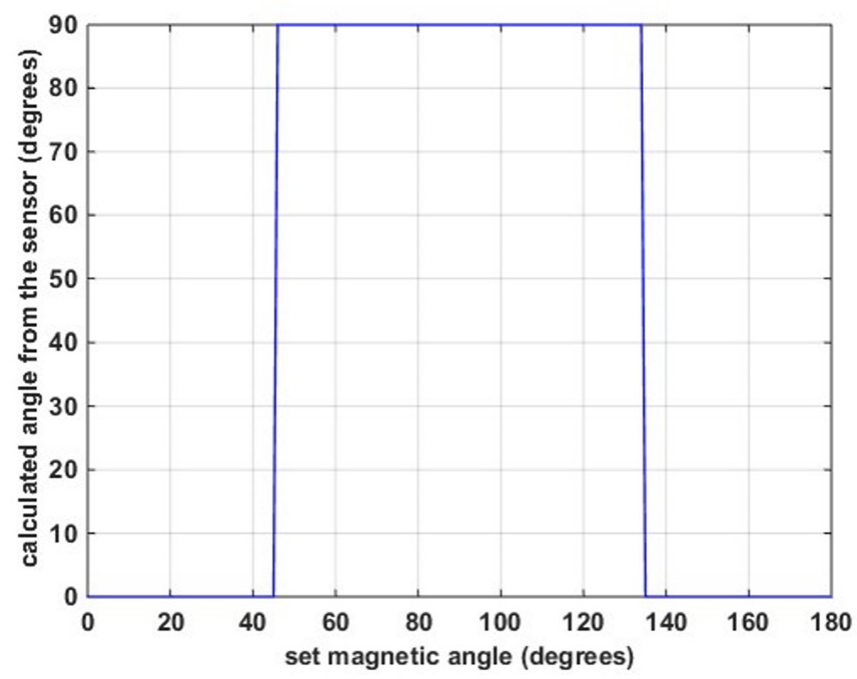

Figure 5.10: Calculated angle from the sensor outputs in case of a broken connection to the power supply or ground in bridge 2 .

One more cause of catastrophic faults in AMR sensors is the loss of the magnetic field which angle is calculated to measure a rotating angle. In automotive applications, the magnetic field is usually generated by a permanent magnet, as shown in Figure 5.11. It represents a setup that can be used to measure the angular position of a rotating shaft from the movement of a permanent magnet fixed to it. Without the magnet, there is no magnetic field $(\mathrm{H})$ to be followed by the magnetisation vector (M) of the sensor, and hence the angle cannot be calculated [Lay08], [Die00] [Kab05]. 


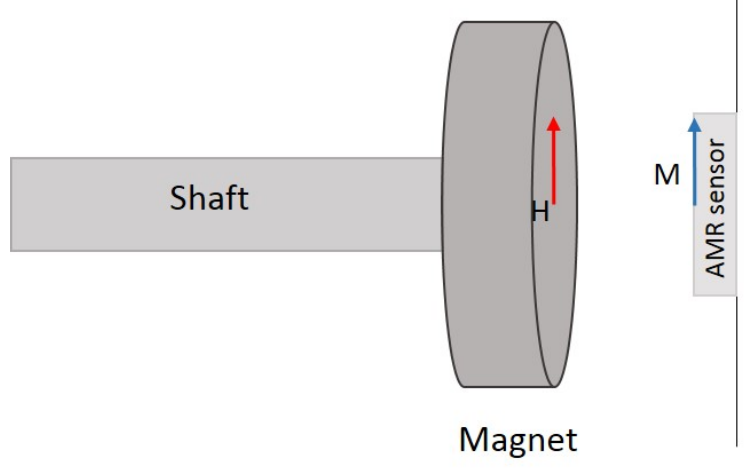

Figure 5.11: Setup to measure the angular position of a rotating shaft using an AMR sensor. $\mathrm{H}$ represents the magnetisation vector of the magnetic field and $\mathrm{M}$ the magnetisation vector of the sensor.

In principle, an AMR sensor must be taken out of operation if any of the catastrophic faults presented above occurs because the angle cannot be longer reliably computed. However, in the following, a fault-tolerant system is proposed that aims to allow the sensor to continue to operate reliably despite faulty bridge resistances or broken bridge connections to the power supply or ground.

\subsection{Fault-tolerant system for AMR sensors}

\subsubsection{Introduction}

In safety-critical systems, reliability and safety are taken into consideration during design, test as well as manufacturing of the system; however certain faults cannot be avoided. Therefore, these faults should be tolerated in such a way that they do not represent a risk to people or the environment. This is usually achieved by incorporating fault-tolerant techniques aimed to preserve the system functionality although a fault condition occurs over time [Abd06], [Ise02]. Most of the fault-tolerant systems that have been proposed are based on redundancy. This concept implies that there is more than one way of performing the required function as detailed in chapter 2 .

A fault-tolerant system generally requires hardware and/or software mechanisms to detect a fault, determine its location and subsequently recover the system operation. Fault-detection and fault-location can be performed via online monitoring during the system operation by comparing specific signals with nominal values to detect an abnormal condition [Sie96]. Once a fault is detected, a recovery process is invoked in which first a fault-reconfiguration eliminates the faulty element by modifying or updating the system configuration. Then the fault-recovery step restores the operational state of the system [Cha09]. In addition, it is possible to perform fault-diagnosis to determine the type of fault present, which is not always required 
but is useful to have more detailed information on the status of the system [Sin11].

In the following, a fault-tolerant system for AMR sensors used for angle measurements is proposed. Our system is capable of handling a faulty resistance (short or open condition) in any of the half-bridges of the sensor or two faulty resistances in one half-bridge (one resistance with an open condition and the other in short condition), but not in different half-bridges. This means that the system tolerates one faulty half-bridge but not two faulty half-bridges at the same time. Furthermore, the sensor can continue its operation despite broken bridge supply-power connections or broken bridge ground connections, assuming that two power supplies are available, as should be the case in a dependable system. If there is a faulty half-bridge and broken bridge connection to the power supply or ground at the same time, the fault-tolerant system first handles the broken connection and once this is recovered, it can deal with the faulty half-bridge.

\subsubsection{Proposed fault-tolerant system}

Our proposed fault-tolerant system executes fault detection, location, reconfiguration and recovery to tolerate catastrophic faults in AMR sensors. The fault-detection is performed by verifying the output voltages of the half-bridges, which in fault-free condition should show values close to half of the power supply plus an AC signal resulting from the magnetoresistance effect as has been shown in Figures 5.2 and 5.3. However, under faulty conditions the affected half-bridge or half-bridges show an output voltage which is either close to zero or close to the value of the power supply, as depicted in Figure 5.12. We have defined that a catastrophic fault is detected in half-bridges with voltages lower that $10 \%$ of the power supply or larger that $90 \%$ of the power supply. Fault-location is performed based on what half-bridge or half-bridges show abnormal output voltages as detailed below.

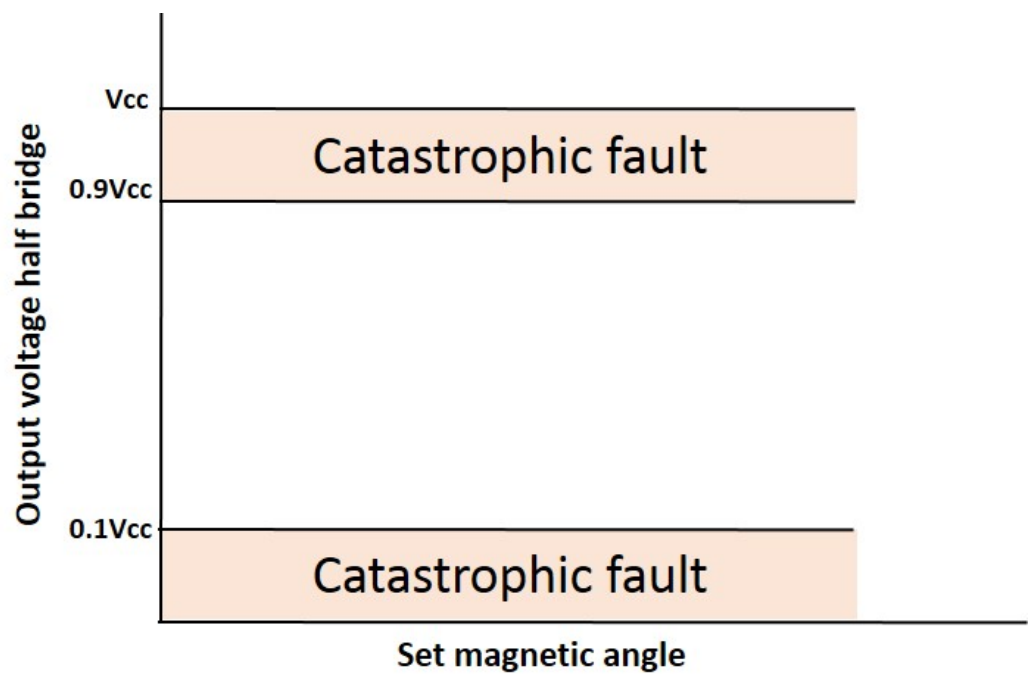

Figure 5.12: Output voltage of a half-bridge in faulty conditions. 
With respect to fault-reconfiguration in case of faulty resistances, this is performed via an analytical redundancy defined based on the following assumptions. First, the output voltages of the half-bridges show cosine and sine signals as presented in Figures 5.2 and 5.3. These signals show a phase shift of $90^{\circ}$ taking into consideration the angle sensed by the sensor, but $45^{\circ}$ for the set magnetic angle, due to the sensor detects two times the magnetic angle because of its configuration. Second, in trigonometry, the sine and cosine of the addition or subtraction of two angles are defined as presented in Equations (5.4) and (5.5) for the angles $\alpha$ and $\beta$.

$$
\begin{aligned}
& \cos (\alpha \pm \beta)=\cos (\alpha) * \cos (\beta) \mp \sin (\alpha) * \sin (\beta) \\
& \sin (\alpha \pm \beta)=\sin (\alpha) * \cos (\beta) \pm \sin (\beta) * \cos (\alpha)
\end{aligned}
$$

Therefore, the output voltages of the half-bridges can be combined to calculate the sine and cosine of the addition of the angles $45^{\circ}$ and $2 \theta$ as shown in Equations (5.6) and (5.7).

$$
\begin{aligned}
& \cos \left(45^{\circ}+2 \theta\right)=\cos \left(45^{\circ}\right) * \cos (2 \theta)-\sin \left(45^{\circ}\right) * \sin (2 \theta) \\
& \sin \left(45^{\circ}+2 \theta\right)=\sin \left(45^{\circ}\right) * \cos (2 \theta)+\cos \left(45^{\circ}\right) * \sin (2 \theta)
\end{aligned}
$$

Figures 5.13 and 5.14 show the output voltages obtained using the original configuration of the sensor in a fault-free condition, as well as combining the voltages of the half-bridges in case of faulty conditions in the bridge resistances. These last output voltages should be described as provided in Equations (5.8) and (5.9).

$$
\begin{aligned}
& V 1=\frac{\sqrt{2} \cdot a m r 1}{2} \times \cos \left(45^{\circ}+2 \theta\right) \\
& V 2=\frac{\sqrt{2} \cdot a m r 2}{2} \times \sin \left(45^{\circ}+2 \theta\right)
\end{aligned}
$$

The angle calculation using the voltages given in Equations (5.8) and (5.9) is described as presented in Equation (5.10), in which an additional $45^{\circ}$ is included as compared to Equation (5.3). However, because of the $45^{\circ}$ phase is known in advance (Figures 5.13 and 5.14), it can be compensated and the angles can still be calculated even if catastrophic faults (open/short condition) occur in the bridge resistances of the sensor. The sine and cosine signals show smaller amplitudes compared with the sensor outputs in fault-free condition. Nevertheless, this does not affect the angle calculation as it depends on the relationship between the signals and not on their amplitudes.

$$
2 \theta=\arctan \left(\frac{V 2}{V 1}\right)-45^{\circ}
$$




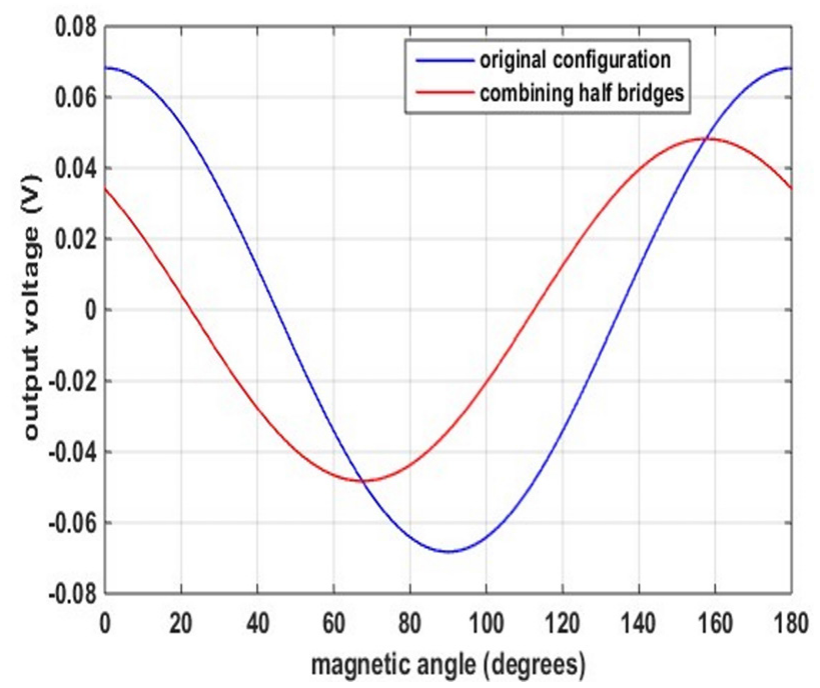

Figure 5.13: Output voltages of bridge 1 considering sensor outputs in the original configuration (fault-free condition) and combining the output voltages of the half-bridges in case of faulty bridge resistances.

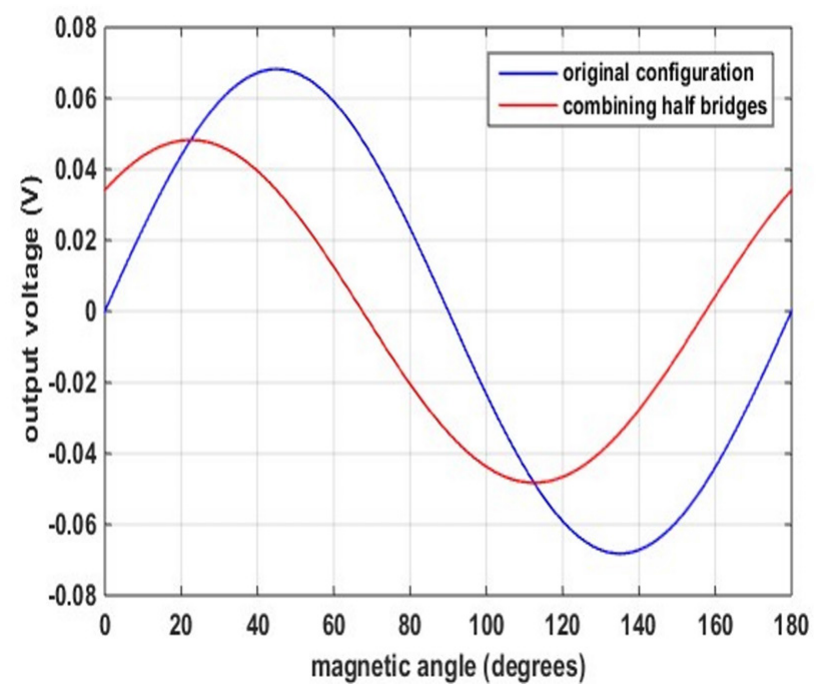

Figure 5.14: Output voltages of bridge 2 considering sensor outputs in the original configuration (fault-free condition) and combining the output voltages of the half-bridges in case of faulty bridge resistances.

The proposed fault-tolerant system considers four possible scenarios for faulty resistances, which have been defined taking into consideration Equations (5.6) and (5.7), but also the number of half-bridges in an AMR sensor. In each scenario, a method for fault-detection, fault-location, fault-reconfiguration and fault-recovery is included. These are presented below according to the Wheatstone bridges shown in Figure 5.1. 
The system has been simulated in MATLAB using the following resistance values. Based on the data included in the datasheet of the AMR sensor KMZ49 [NXP11], we have defined that a fault-free resistance varies between $3000 \Omega$ and $3083 \Omega$ depending on the magnetic angle. A resistance with a short condition has been represented as

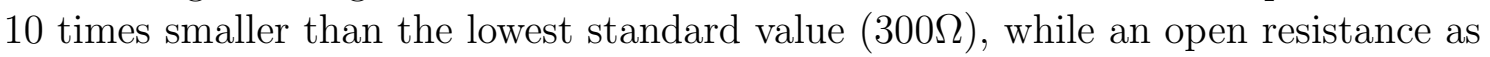
10 times bigger $(30 \mathrm{k} \Omega$ ). The voltage of the power supply (VCC) has been defined at $5 \mathrm{~V}$; hence a catastrophic fault is determined in half-bridges with output voltages less than $0.5 \mathrm{~V}$ or higher than $4.5 \mathrm{~V}$. The results presented regarding the calculated angles with the proposed reconfigurations have been compensated for the $45^{\circ}$ phase mentioned above.

\section{Scenario one}

In this scenario, a fault condition in half 1 of bridge 1 is considered, which could occur due to short or open conditions in resistances $\mathrm{R} 1$ or/and R4. This results in an abnormal output voltage in vop1 as shown in Table 5.1, which can be used for fault-detection and fault-location.

Table 5.1: Sensor conditions, scenario one.

\begin{tabular}{|l|c|}
\hline Sensor Condition & $\boldsymbol{v o p} 1$ \\
\hline Fault-free & $\mathrm{VCC} / 2 \pm \mathrm{AMR}$ \\
$\mathrm{R} 1$ (short) or/and R4 (open) & $\approx 0$ \\
$\mathrm{R} 1$ (open) or/and R4 (short) & $\approx \mathrm{VCC}$ \\
\hline
\end{tabular}

VCC: power supply voltage; AMR: voltage because of magnetoresistance effect

In case of faulty resistances in half 1 of bridge 1 , the fault-reconfiguration combines the output voltage of half 2 of bridge 1 and the output of the two half-bridges of bridge 2 as presented in Equations (5.11) and (5.12), and thus fault-recovery is performed.

$$
\begin{aligned}
& V 1=\operatorname{von} 2-\operatorname{von} 1=\frac{\sqrt{2} \cdot a m r 1}{2} * \cos \left(45^{\circ}+2 \theta\right) \\
& V 2=\operatorname{vop} 2-\operatorname{von} 1=\frac{\sqrt{2} \cdot a m r 2}{2} * \sin \left(45^{\circ}+2 \theta\right)
\end{aligned}
$$

For simulation purposes, a short condition in resistance $\mathrm{R} 1$ has been considered that results in an output voltage less than $0.5 \mathrm{~V}$ at vop 1, as presented in Figure 5.15. In this figure, the output voltage of the other three half-bridges are also included, which show the expected values close to half of the power supply plus the value resulting from the magnetoresistance effect as shown in Figures 5.2 and 5.3. 


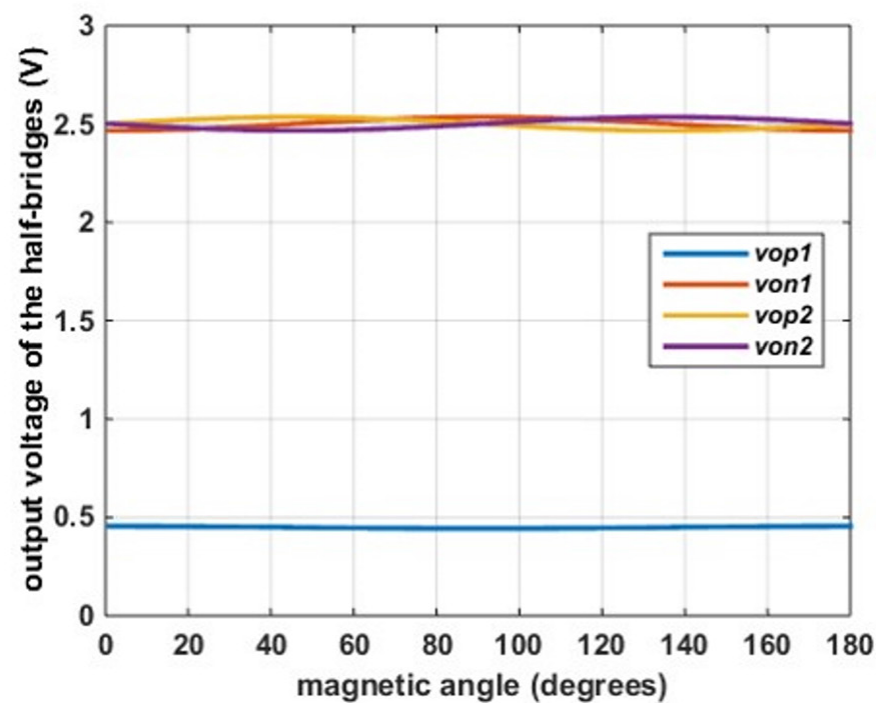

Figure 5.15: Output voltages of the half-bridges in scenario one. A schematic of an AMR sensor in which vop1, von1, vop2 and von2 are presented is shown in Figure 5.1.

Figure 5.16 shows the calculated angles with the original sensor outputs, as well as with the proposed reconfiguration in scenario one. While with the original configuration the angle obtained oscillates between $\pm 90^{\circ}$, by applying the reconfiguration it is still possible to calculate the angle.

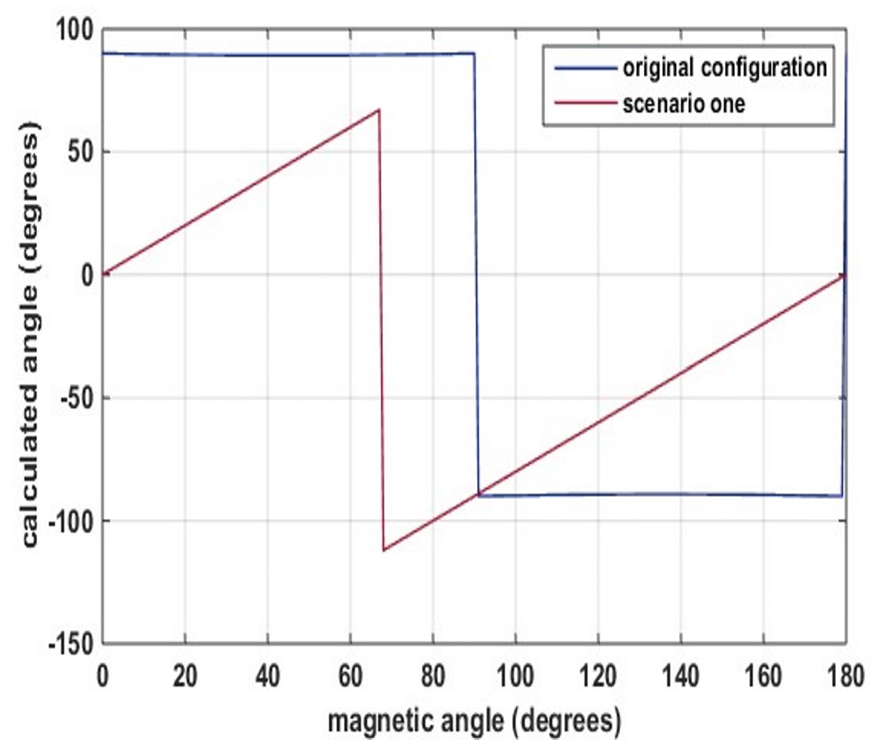

Figure 5.16: Calculated angles in scenario one assuming a short condition in resistance R1 of the Wheatstone bridges shown in Figure 5.1. The original configuration represents the calculated angles using the sensor outputs assuming a fault-free condition, while for scenario one the voltages specified in the fault-reconfiguration are used. 


\section{Scenario two}

A faulty condition in half 2 of bridge 1 can occur due to short or open conditions in the resistances R2 or/and R3, which brings as consequence an abnormal output voltage in von1. Therefore, the fault-detection and fault-location is performed based on the voltage of von 1 that varies as shown in Table 5.2.

Table 5.2: Sensor condition, scenario two.

\begin{tabular}{|l|c|}
\hline Sensor Condition & von1 \\
\hline Fault-free & $\mathrm{VCC} / 2 \pm \mathrm{AMR}$ \\
$\mathrm{R} 2$ (short) or/and R3(open) & $\approx 0$ \\
$\mathrm{R} 2$ (open) or/and R3(short) & $\approx \mathrm{VCC}$ \\
\hline
\end{tabular}

VCC: power supply voltage; AMR: voltage because of magnetoresistance effect

Regarding the fault-reconfiguration and fault-recovery, the angle can still be calculated using the output voltages of the other three half-bridges, as depicted in Equations (5.13) and (5.14).

$$
\begin{aligned}
& \text { vout } 1=\operatorname{vop} 1-\operatorname{vop} 2=\frac{\sqrt{2} \cdot a m r 1}{2} * \cos \left(45^{\circ}+2 \theta\right) \\
& \text { vout } 2=\operatorname{vop} 1-\operatorname{von} 2=\frac{\sqrt{2} \cdot a m r 2}{2} * \sin \left(45^{\circ}+2 \theta\right)
\end{aligned}
$$

This scenario has been simulated considering an open condition in resistance $\mathrm{R} 3$ of half 2 in bridge 1 . As a result, the voltage at von 1 is less than $0.5 \mathrm{~V}$ while the other three half-bridges present nominal output voltages like in the fault-free condition, as is shown in Figure 5.17. 


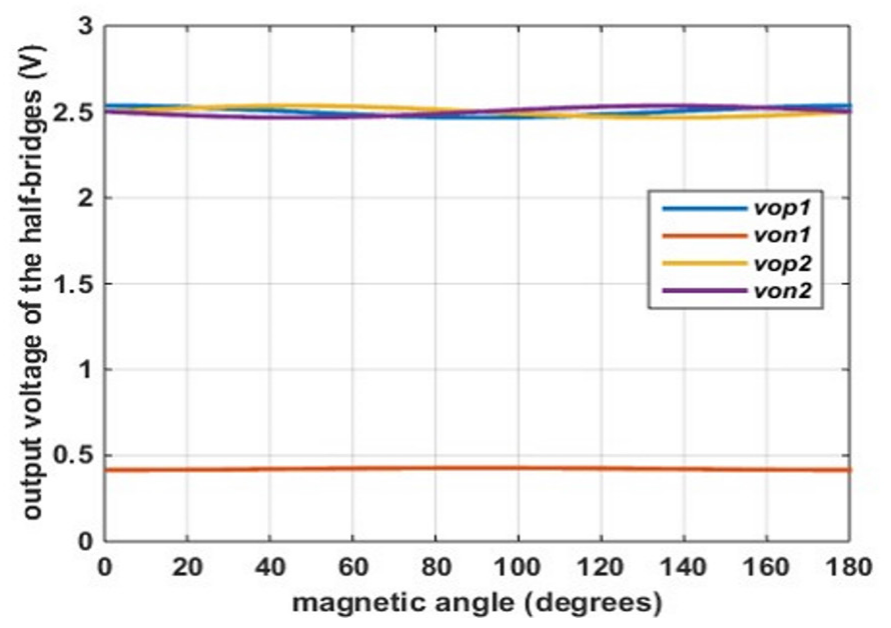

Figure 5.17: Output voltages of the half-bridges in scenario two. A schematic of an AMR sensor in which vop1, von1, vop2 and von2 are presented is shown in Figure 5.1.

In terms of fault-reconfiguration and fault-recovery, Figure 5.18 shows the calculated angles with the sensor outputs in the original configuration as well as with the reconfiguration specified in this scenario. While with the original configuration the angle is always close to $0^{\circ}$ irrespective of the magnetic angle, with the voltages used after reconfiguration it is still possible to calculate the angle.

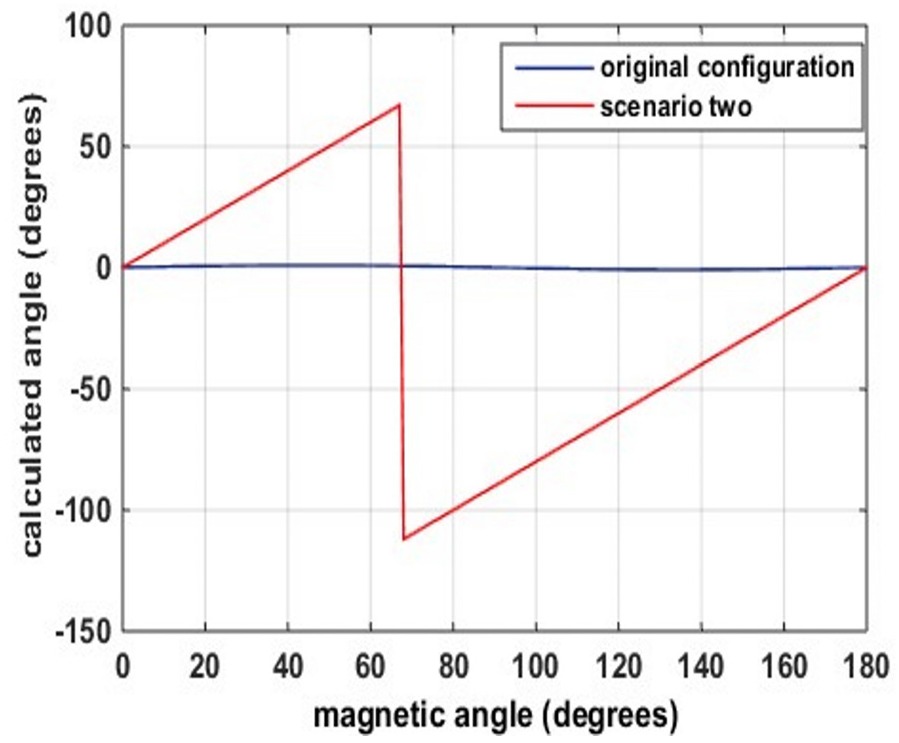

Figure 5.18: Calculated angles in scenario two assuming an open condition in resistance R3 of the Wheatstone bridges shown in Figure 5.1. The original configuration represents the calculated angles using the sensor outputs assuming a fault-free condition, while for scenario two the voltages specified in the fault-reconfiguration are used. 


\subsection{Fault-tolerant system for AMR sensors}

\section{Scenario three}

This scenario refers to short or open conditions in resistances R21 or/and R24 of half 1 in bridge 2 . Then, fault-detection and fault-location can be performed by verifying the output voltage of vop2, which varies as shown in Table 5.3.

Table 5.3: Sensor condition, scenario three

\begin{tabular}{|l|c|}
\hline Sensor Condition & vop2 \\
\hline Fault-free & $\mathrm{VCC} / 2 \pm \mathrm{AMR}$ \\
$\mathrm{R} 21$ (short) or R24 (open) & $\approx 0$ \\
R21(open) or R24 (short) & $\approx \mathrm{VCC}$ \\
\hline
\end{tabular}

VCC: power supply voltage; AMR: voltage because of magnetoresistance effect

In fault-reconfiguration, the output voltage of the other half-bridges are combined, as shown in Equations (5.11) and (5.14). Therefore, the operational state of the sensor is restored.

For simulation purposes, an open condition in resistance R21 has been considered. As shown in Figure 5.19, this bring as consequence a voltage greater than $4.5 \mathrm{~V}$ in vop2, while the other three half-bridges show nominal output values. Due to the abnormal voltage in vop2, the calculated angle with the original configuration is always close to $45^{\circ}$; but with the voltages proposed in fault-reconfiguration it is possible to follow the magnetic angle, as presented in Figure 5.20.

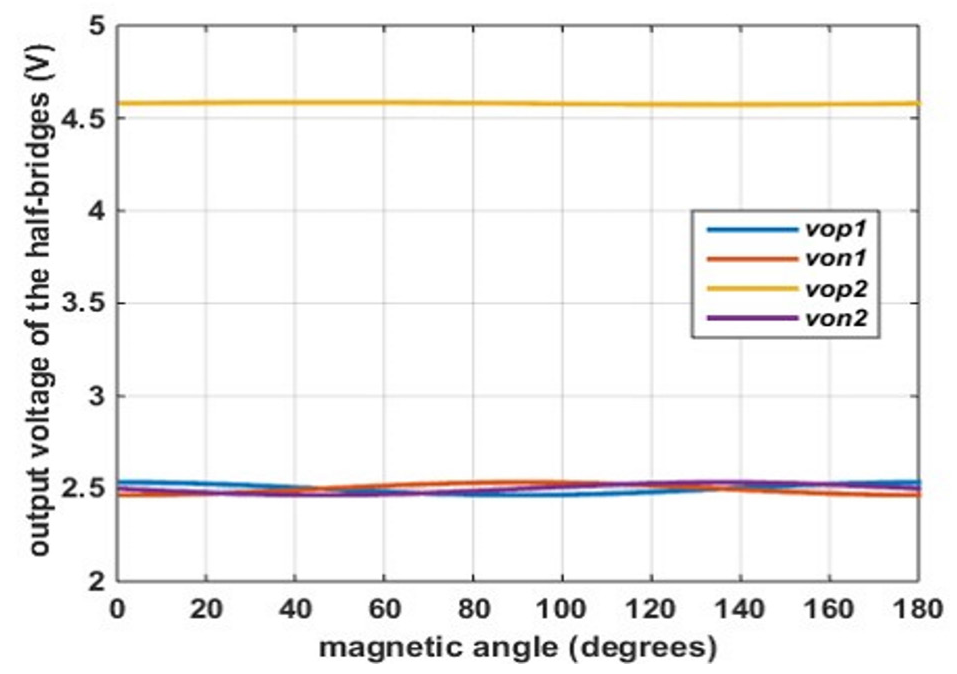

Figure 5.19: Output voltages of the half-bridges in scenario three. A schematic of an AMR sensor in which vop1, von1, vop2 and von2 are presented is shown in Figure 


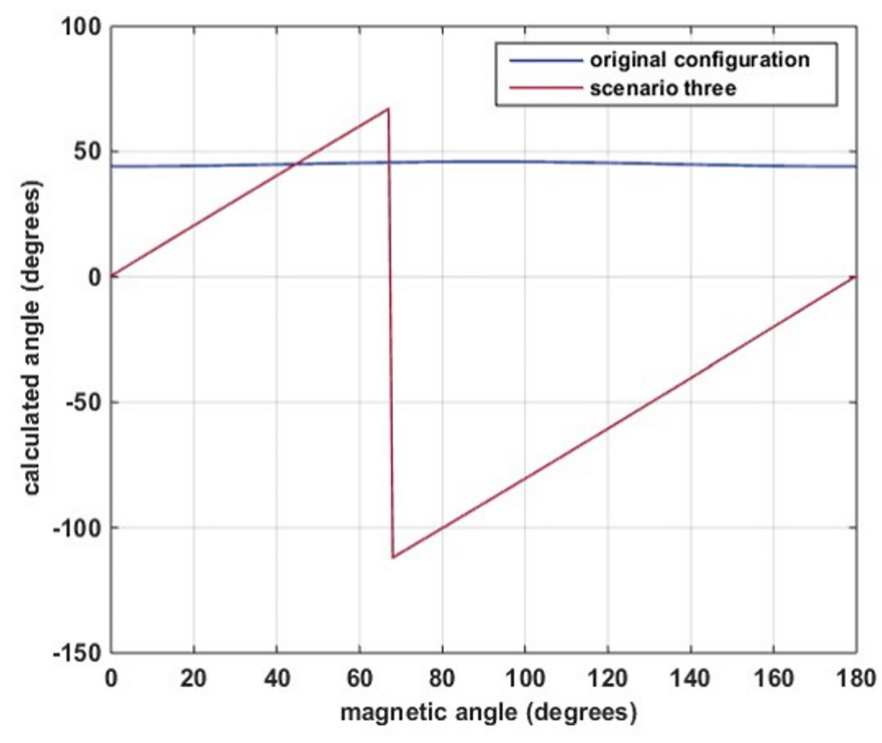

Figure 5.20: Calculated angles in scenario three assuming an open condition in resistance R21 of the Wheatstone bridges shown in Figure 5.1. The original configuration represents the calculated angles using the sensor outputs assuming a fault-free condition, while for scenario three the voltages specified in the fault-reconfiguration are used.

\section{Scenario four}

This scenario focusses on faulty conditions (short/open) in the resistances of half 2 of bridge 2 . The fault-detection and fault-location are based on verifying the output voltage of the half-bridges to detect an abnormal voltage in von2, which varies as specified in Table 5.4. Regarding fault-reconfiguration and fault-recovery, the angle can still be calculated by combining the output voltage of the other half-bridges as presented in Equations (5.12) and (5.13).

Table 5.4: Sensor condition, scenario four

\begin{tabular}{|l|c|}
\hline Sensor Condition & von2 \\
\hline Fault-free & $\mathrm{VCC} / 2 \pm \mathrm{AMR}$ \\
$\mathrm{R} 22$ (short) or/and R23 (open) & $\approx 0$ \\
$\mathrm{R} 22$ (open) or/and R23 (short) & $\approx \mathrm{VCC}$ \\
\hline
\end{tabular}

VCC: power supply voltage; AMR: voltage because of magnetoresistance effect

In simulation, an open condition in resistance R22 was assumed which affects the output voltage of von2, showing a value greater than $4.5 \mathrm{~V}$, as depicted in Figure 5.21. The other three half-bridges show nominal values like under the fault-free condition. 


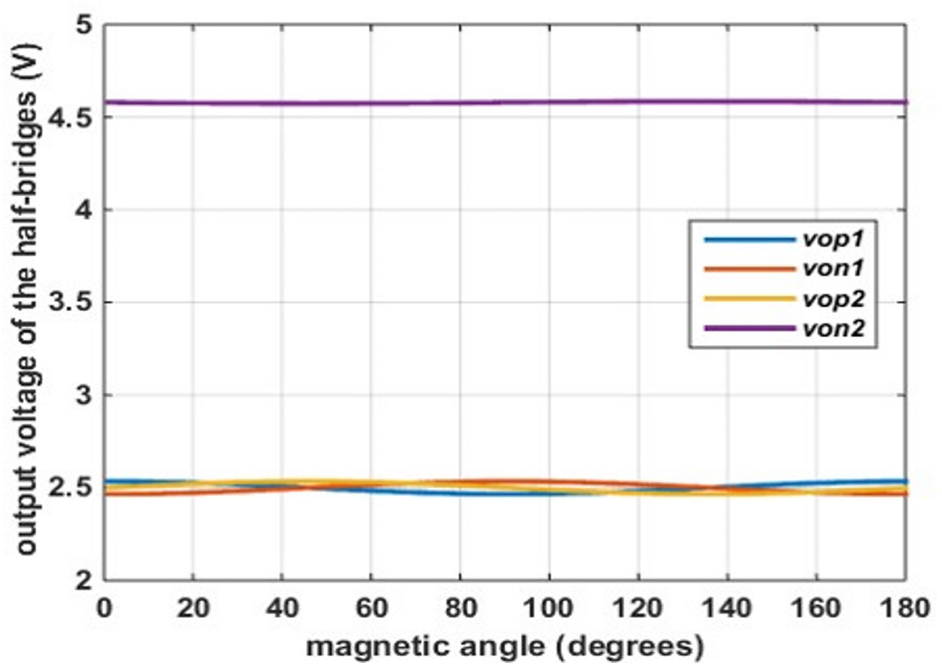

Figure 5.21: Output voltages of the half-bridges in scenario four. A schematic of an AMR sensor in which vop1, von1, vop2 and von2 are presented is shown in Figure 5.1 .

In case of catastrophic faults in the resistances of half 2 of bridge 2 , the calculated angle with the original configuration of the sensor is always close to $-45^{\circ}$ irrespective of the magnetic angle. On the other hand, by fault-reconfiguration and fault-recovery it is possible to calculate the angle, as presented in Figure 5.22.

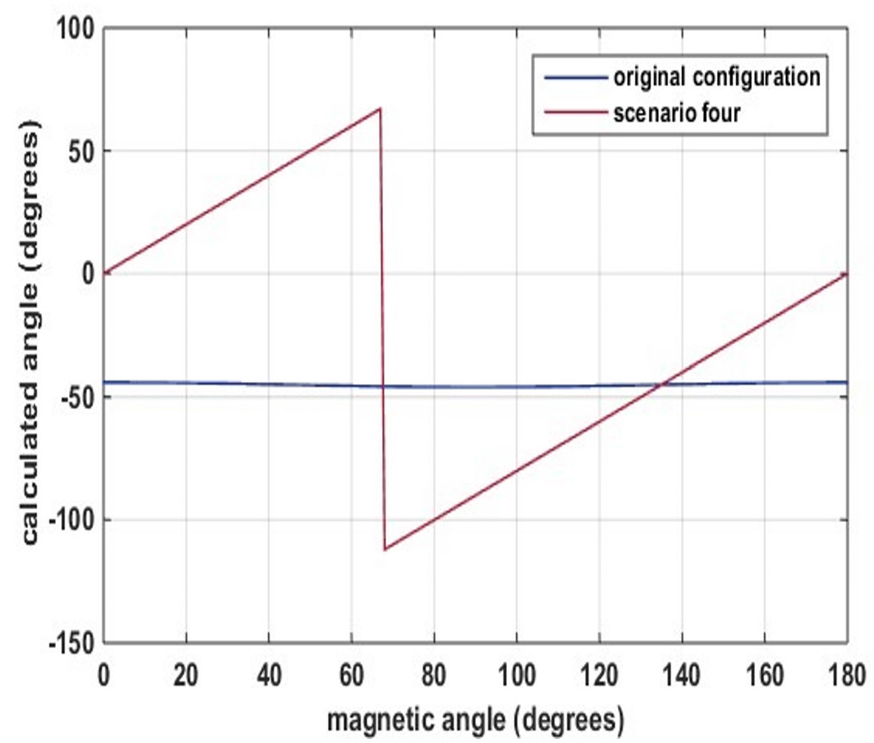

Figure 5.22: Calculated angles in scenario four assuming an open condition in resistance R22 of the Wheatstone bridges shown in Figure 5.1. The original configuration represents the calculated angles using the sensor outputs assuming a fault-free condition, while for scenario four the voltages specified in the fault-reconfiguration are used. 
In summary, the results presented above show that the proposed fault-tolerant system allows an AMR sensor for angle measurements to continue operating despite the occurrence of catastrophic faults in the bridge resistances of the sensor. In all the scenarios, the sensor outputs in the original configuration cannot be longer used as the angle cannot be reliably computed. On the other hand, the proposed system is able to detect and locate faulty conditions by monitoring the voltage of the half-bridges of the sensor. Once a fault is determined, a reconfiguration is applied based on analytical redundancy to obtain new output voltages that allow calculating the magnetic angle and restore the operational state of the sensor. As mentioned above, the fault-tolerant system is capable of handling a faulty half-bridge with one faulty resistance (short or open condition) or two faulty resistances (one in short condition and the other in open condition), but it is not possible to tolerate two faulty half-bridges at the same time. Therefore in case of two faulty resistances in different half-bridges, the sensor must be taken out of operation. Although the amplitudes of the sinusoidal signals obtained from fault-reconfiguration are smaller compared to the signals of the sensor outputs in the original configuration, this does not affect the angle calculation as this depends on the relationship between the sine and cosine signals, which remains the same.

The proposed reconfigurations in the four scenarios provide the same results in terms of angles calculated as is shown in Figure 5.23 in which the angles calculated in fault-free conditions are also included. The calculations have been performed using sensor outputs measured in a commercial AMR sensor KMZ49. In case a linear range between 0 to $180^{\circ}$ is required, then if the calculated angle is negative $180^{\circ}$ should be added to the calculated angle as presented in Equation (5.15).

$$
\text { angle }_{\text {compensated }}=180^{\circ}+\text { angle }_{\text {negative }}
$$



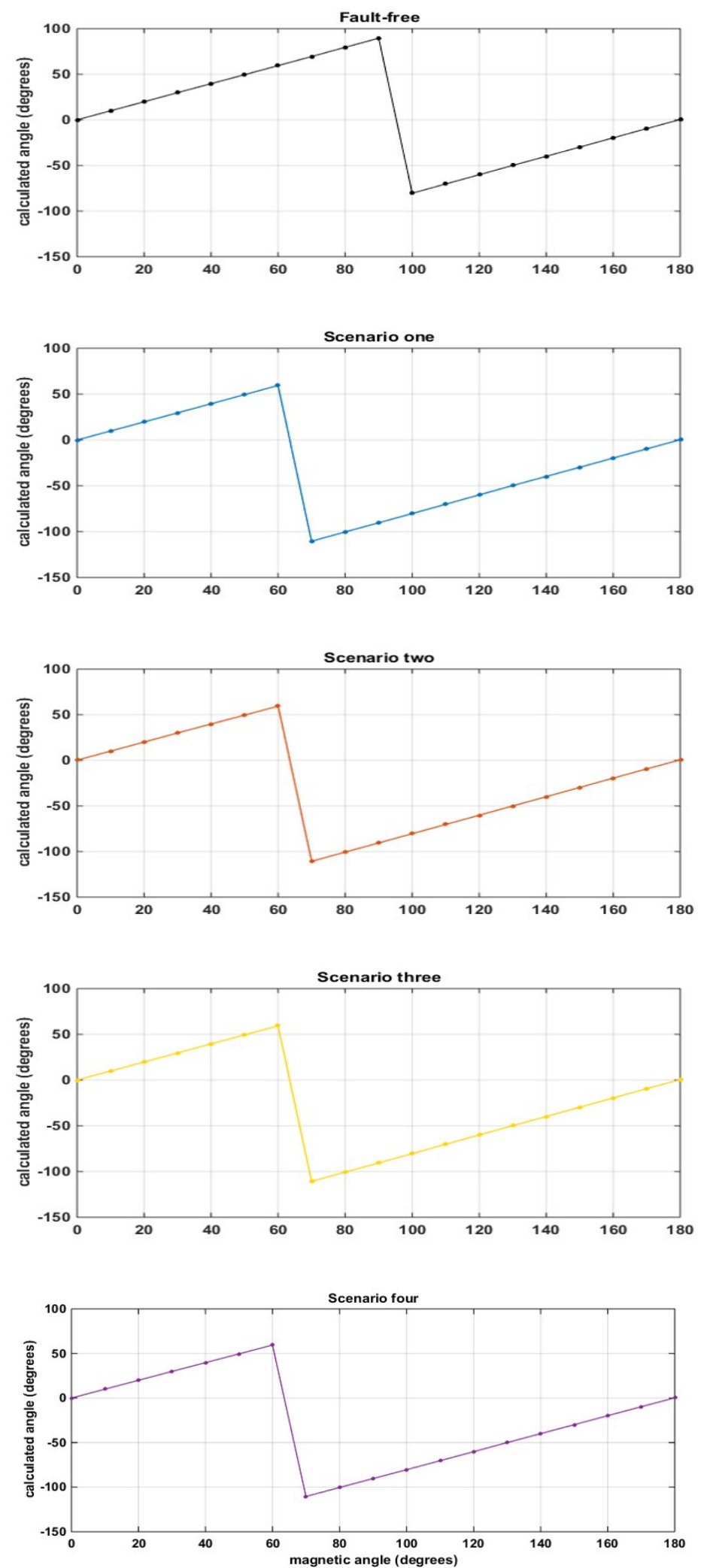

Figure 5.23: Calculated angles using voltages measured in a commercial sensor KMZ49 and considering the output voltages in fault-free conditions as well as in the four scenarios for the fault-tolerant system. 
Now, regarding catastrophic faults because of broken bridge connections to the power supply or ground. These can be detected by the abnormal output voltages at the half-bridges (vop, von) of the affected Wheatstone bridge, as shown in Table 5.5. In case of a broken bridge power supply connection, both half-bridges show small output voltages, while for a broken ground connection the output voltages are close to the value of the power supply.

Table 5.5: Output voltage of the half-bridges versus the sensor condition

\begin{tabular}{|l|c|c|}
\hline Sensor Condition & $\boldsymbol{v o p}$ & $\boldsymbol{v o n}$ \\
\hline Fault-free & $\mathrm{VCC} / 2 \pm \mathrm{AMR}$ & $\mathrm{VCC} / 2 \pm \mathrm{AMR}$ \\
Broken bridge power supply connection & $\approx 0$ & $\approx 0$ \\
Broken bridge ground connection & $\approx \mathrm{VCC}$ & $\approx \mathrm{VCC}$ \\
\hline
\end{tabular}

VCC: power supply voltage; AMR: voltage due to magnetoresistance effect.

Fault-reconfiguration and fault-recovery have been defined taking into consideration that two power supplies (supply1, supply2) are available together with a set of switches that establish their connections to the Wheatstone bridges, as shown in Figure 5.24. This assumption is valid because in dependable systems a single point of failure should be avoided, so a backup power supply must also be included. Once a broken connection is detected in one bridge, then during fault-reconfiguration a switch-over to the other power supply is performed to recover the functionality of the sensor. Additionally, a fault condition in the power supply that is being used can also be detected based on the abnormal output voltages in all half-bridges, so a switch-over to the other power supply can be performed.

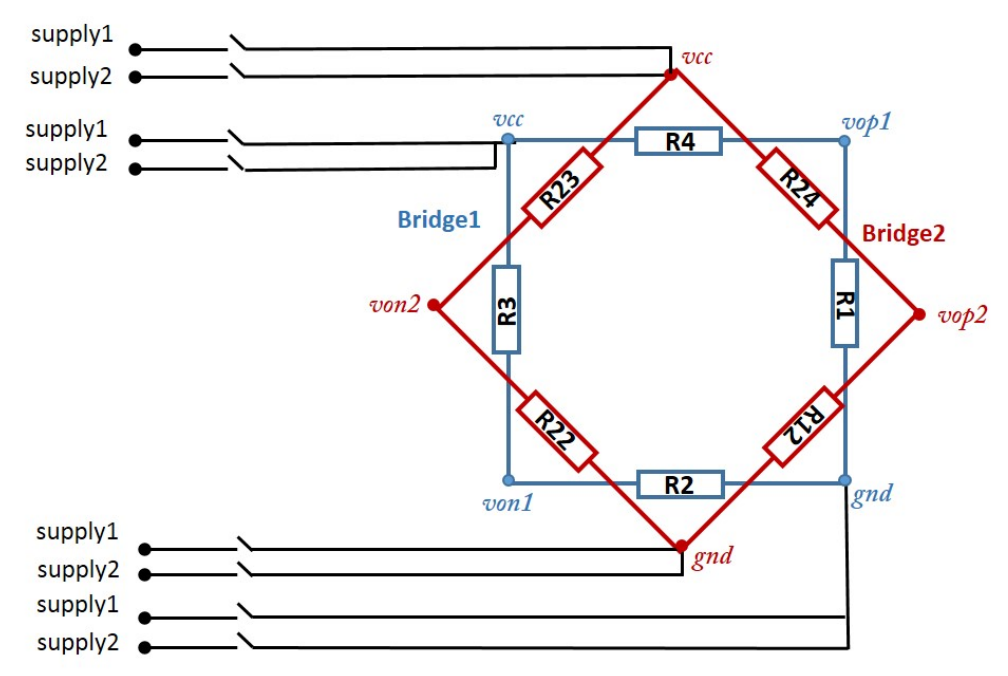

Figure 5.24: Schematic of an AMR sensor with the connections to two power supplies included. 
Furthermore, it is also possible to detect the loss of the magnet that generates the magnetic field which angle is to be measured. In this case, the four half-bridges show output voltages approximately equal to half of the voltage of the power supply, bringing as consequence that the differential outputs of both bridges are approximately zero at the same time. This does not occur under normal conditions because of the characteristics of the sinusoidal signals resulting from the magnetoresistance effect. In this event, the sensor must be taken out of operation because it has lost the connection with the angle to be measured.

Regarding fault-diagnosis, an analysis of the output voltages of the sensor allows determining the source of failure as shown in Table 5.6, which refers to one bridge, but the same applies for both Wheatstone bridges of an AMR sensor. A voltage close to the value of the power supply or close to ground in the two half-bridges of one bridge means an issue with its connection to the power supply or ground. If both bridges show at the same time differential output voltages close to zero and in the half-bridges values close to half the power supply, then the connection to the magnet has been lost. An abnormal output voltage in a half-bridge that makes the differential output of the bridge to include an extra value (VCC/2) refers to a fault condition in one of the bridge resistances. The possible reason for the faulty resistance can be determined based on the Tables 5.1, 5.2, 5.3, and 5.4.

Table 5.6: Fault-diagnosis based on the output voltages of an AMR sensor. The table refers to the output voltages of one bridge but the same applies for the two bridges in the sensor.

\begin{tabular}{|l|c|c|c|}
\hline Sensor Condition & $\boldsymbol{v o p}$ & $\boldsymbol{v o n}$ & $\begin{array}{c}\text { vout } \\
\text { vop } \text {-von })\end{array}$ \\
\hline Fault-free & $\mathrm{VCC} / 2 \pm \mathrm{AMR}$ & $\mathrm{VCC} / 2 \pm \mathrm{AMR}$ & $2 . \mathrm{AMR}$ \\
\hline $\begin{array}{l}\text { Broken bridge } \\
\text { supply connection }\end{array}$ & $\approx 0$ & $\approx 0$ & $\approx 0$ \\
\hline $\begin{array}{l}\text { Broken bridge } \\
\text { ground connection }\end{array}$ & $\approx \mathrm{VCC} / 2$ & $\approx \mathrm{VCC} / 2$ & $\approx 0$ \\
\hline Magnet lost & $\mathrm{X}$ & $\mathrm{VCC} / 2 \pm \mathrm{AMR}$ & $\mathrm{VCC} / 2 \pm \mathrm{AMR}$ \\
\hline $\begin{array}{l}\text { Faulty resistance } \\
\text { half-bridge } 1\end{array}$ & $\mathrm{VCC} / 2 \pm \mathrm{AMR}$ & $\mathrm{X}$ & $\mathrm{VCC} / 2 \pm \mathrm{AMR}$ \\
\hline $\begin{array}{l}\text { Faulty resistance } \\
\text { half-bridge } 2\end{array}$ & & & \\
\hline
\end{tabular}

VCC: power supply voltage; AMR: voltage due to magnetoresistance effect; $\mathrm{X}: \approx 0$ or $\approx \mathrm{VCC}$ depending on the faulty resistance; half-bridge 1 and 2 are based on Figure 5.1 


\subsection{Conclusions}

In principle, an AMR sensor for angle measurements must be taken out of operation in case of catastrophic faults because the angle can no longer be reliably computed. Catastrophic faults can be caused by an open or short condition in the bridge resistances, broken bridge connections to the power supply or ground, but also because of the loss of the magnet that generates the magnetic field, which angle is to be measured. Therefore, fault-tolerant techniques aimed to allow the sensor to continue its operation despite the occurrence of catastrophic faults are usually included, especially in safety-critical applications. So far, most of the fault-tolerant systems that have been proposed can handle faulty resistances based on physical redundancy, either adding extra sensors of the same type like in [Die00] or adding extra sensors with another operating principle as in [Dil03]. However, the main drawback of physical redundancy is the additional hardware involved, which can be restricted by space limitations in cars and also increases the production costs, being both critical factors for mass production in the automotive industry.

This chapter focussed on proposing a fault-tolerant system capable of tolerating faulty bridge resistances as well as broken bridge connections to the power supply or ground in AMR sensors used for angle measurements. The faulty conditions are handled by performing fault-detection and fault-location and then fault-reconfiguration to recover the sensor operation. Compared with the methods mentioned above, our system applies analytical redundancy for fault-reconfiguration in case of faulty resistances, which means that no additional sensors are required. The redundancy is based on the fact that the sensor is redundant by nature, as it includes four halfbridges in which faulty resistances generate faulty half-bridge or half-bridges. The angle is calculated from the output voltage of the half-bridges, so in case of a faulty half-bridge, the output voltages of the three fault-free half-bridges are reconfigured depending on their sinusoidal characteristics to obtain sensor outputs that can still be used to calculate the angle and hence recover the operational state of the sensor.

The proposed system can tolerate a half-bridge with one faulty resistance (short or open condition) or two faulty resistances (one in short condition and the other in open condition). The calculated angles include an additional $45^{\circ}$ phase shift due to the $45^{\circ}$ phase of the sinusoidal signals obtained by reconfiguration. However, because this is known in advance, it can be compensated to calculate the correct angles even in case of faulty resistances in the sensor. This has been verified by MATLAB simulation, using as input the data obtained from an analytical model of an AMR sensor but also from measurements of commercial AMR sensors. In the event of broken bridge connections to the power supply or ground, the fault-reconfiguration is performed by switching over to a backup power supply, which can also be applied if a fault occurs in the active power supply. This procedure is valid because in dependable systems a backup power supply should be included.

The fault-detection and fault-location steps executed before applying the necessary reconfiguration are based on the abnormal output voltage caused by the faulty 
condition. In a fault-free state, the output voltages of the half-bridges should be half of the value of the power supply plus a value resulting from the magnetoresistance effect. On the other hand, in case of faulty resistances or broken bridge connections to the power supply or ground, the voltage of the affected half-bridge or half-bridges shifts close to the value of the power supply or close to ground depending on the fault condition. Additionally, a magnet loss condition can be determined by the fact that the differential output voltage of both bridges are close to ground and the half-bridges are equal to half of the voltage of the power supply. However, in this situation, the sensor should provide a warning and should be taken out of operation while a safe-state is guaranteed.

As mentioned above, our fault-tolerant system is capable of handling one or two faulty resistances in one half-bridge. However, it is not applicable for faulty resistances in different half-bridges, in which case the sensor cannot be longer used. Although having additional sensors would be better in this situation, taking into consideration the low probability of this to happen and the disadvantages of physical redundancy, we consider analytical redundancy as the best option to handle faulty resistances with open or short conditions in the sensor. In case of a faulty half-bridge and broken bridge connection to the power supply or ground at the same time, the fault-tolerant system first takes care of the broken connection and then of the faulty half-bridge. In conclusion, the proposed system allows improving the dependability of AMR sensors used for angle measurements, which becomes fail-operational as required in automotive applications. 


\section{References}

[Abd06] M. Abd-El-Barr. Design and Analysis of Reliable and Fault-Tolerant Computer Systems, 2006.

[Buj04] G. Buja, S. Castellan, R. Menis, and A. Zuccollo. "Dependability of safety-critical systems". In IEEE International Conference on Industrial Technology, volume 3, pp. 1561-1566, Dec 2004.

[Cha09] V. K. Chandhrasekaran and E. Choi. "Fault tolerance for embedded control system". In 9th International Symposium on Communications and Information Technology, pp. 1316-1320, Sept 2009.

[Die00] K. Dietmayer and M. Weser. "Contactless Angle Measurement using KMZ41 and UZZ9000". Application Note AN00023, Philips Semiconductors, Germany, 2000.

[Dil03] E. Dilge, M. Gulbins, T. Ohnesorge, and B. Straube. "On a redundant diversified steering angle sensor". In 9th IEEE On-Line Testing Symposium (IOLTS), pp. 191-196, July 2003.

[Gao15] Z. Gao, C. Cecati, and S. X. Ding. "A Survey of Fault Diagnosis and Fault-Tolerant Techniques Part I: Fault Diagnosis With Model-Based and Signal-Based Approaches". In IEEE Transactions on Industrial Electronics, volume 62(6), pp. 3757-3767, June 2015.

[Inf14] Infineon. "Dual-Sensor Package.Two Sensors in One SMD Packge". Product brief, Infineon Technologies AG, Germany, 2014.

[Ise02] R. Isermann, R. Schwarz, and S. Stolzl. "Fault-tolerant drive-by-wire systems". In IEEE Control Systems, volume 22(5), pp. 64-81, Oct 2002.

[Kab05] P. Kabisatpathy, A. Barua, and S. Sinha. Fault diagnosis of analog integrated circuits, volume 30. Springer Science \& Business Media, 2005.

[Lay08] G. Lay. Fault Tolerant Position Control of SM-PMSM In an ElectroMechanical Brake Actuator Against AMR Angle Sensor Failure. Ph.D. thesis, Technical University of Berlin, Berlin, Oct 2008.

[NXP11] NXP. "KMZ49 datasheet". NXP semiconductors, 2011.

[Run12] A. Runge. "Reliability Enhancement of Fault-prone Many-core Systems Combining Spatial and Temporal Redundancy". In IEEE 14th International Conference on High Performance Computing and Communication, pp. 16001605, June 2012.

[Sie96] M. Sievers. "Fault-tolerance: a methodology for implementing highly dependable systems". In 15th AIAA/IEEE Digital Avionics Systems Conference (DASC), pp. 465-470, Oct 1996. 
[Sin11] P. Sinha. "Architectural design and reliability analysis of a fail-operational brake-by-wire system from ISO 26262 perspectives". Reliability Engineering and System Safety, volume 96(10), pp. 1349 - 1359, 2011.

[Tre01] C. Treutler. "Magnetic sensors for automotive applications". In Third European Conference on Magnetic Sensors and Actuators., volume 91(1-2), pp. 2-6, 2001.

[Zam15] A. Zambrano and H. G. Kerkhoff. "Fault-tolerant system for catastrophic faults in AMR sensors". In 21st IEEE International On-Line Testing Symposium (IOLTS), pp. 65-70, July 2015. 
This page intentionally left blank. 


\title{
Chapter 6
}

\section{A dependable AMR sensor system}

\begin{abstract}
In chapters 4 and 5, self-X properties and a fault-tolerant method have been proposed as means to improve the dependability of AMR sensors used for angle measurements. In this chapter, the architecture of an AMR sensor system that includes these methods together with a module to compensate the sensor outputs in real-time and an IEEE 1687 interface is proposed. The results of the dependability assessment of this system, which has been carried out focussed on the dependability attributes reliability, safety, maintainability and availability are presented.
\end{abstract}

\subsection{Introduction}

Future generations of automobiles will further increase the usage of X-by-wire systems instead of the mechanical and hydraulic parts traditionally used. Although electronic systems offer various benefits regarding performance, comfort, and safety, they also introduce vulnerabilities associated with the electronic components, such as process variation, electrostatic discharge, and performance degradation resulting from aging effects. All these can affect the service delivered by the system and therefore an increasing emphasis has been placed on dependability, especially in safety-critical applications. Dependability can be defined as the ability of a system to perform its intended functions under certain conditions during a period of time [Ker10]. It encompasses several attributes such as reliability, safety, maintainability, and availability as explained in chapter 2 .

X-by-wire systems demand reliable, high-performance and low-cost electronic components that are cable of operating for a long time in a highly dependable manner despite the harsh operating conditions which can be present in vehicles [Tre01]. Designing a dependable system is a tough challenge, especially in the automotive field in which there are strict space and cost constraints but still a high performance is required [Buj04], [Pim04]. The dependability requirements in automotive applications have been presented in chapter 2. These include that the system should be fail-operational, meaning it should continue operating despite the occurrence of

Parts of this chapter have been presented at the 22nd IEEE European Test Symposium (ETS) [Zam17b] and at the International Test Conference Asia (ITC-Asia) [Zam17a] 
a fault; concerning reliability, the goal is to match a failure rate of $10^{-9}$ per hour (1 FIT) that corresponds to a simple mechanical component [Ham03]. In terms of safety, the aim is to meet the ASIL level D of the ISO 26262 functional safety standard that implies a failure rate smaller than $1.10^{-8}$ per hour and it is usually required for safety-critical applications [Sch15]. The standard also enables the design of automotive electronic systems that can prevent dangerous failures and control or minimise them if they occur [Sin11].

Concerning maintainability, the requirement is that the sensor is able to continue its operation after catastrophic faults via repair. In addition, it should be capable of handling performance degradation in such a way that an extreme out-of-range parameter does not lead to a malfunction, as for example an offset voltage that generates a larger angle error than is allowed. With regard to availability, it is defined by the uptime and downtime as mentioned in chapter 2. Although the actual value of these parameters is defined by the application, the goal is that the system operates correctly as long as possible, meaning a large uptime and short downtime.

A traditional AMR sensor for angle measurements basically consists of 8 magnetoresistances configured into two Wheatstone bridges, as explained in chapter 2. It is often used in automotive applications for e.g. measuring the steering angle and motor positioning, which are considered safety-critical applications [NXP11]. However, the sensor is affected by performance degradation due to aging effects as presented in chapter 3; in addition, also catastrophic faults can occur that make the sensor suddenly stop working, which means it cannot be considered fail-operational by default as detailed in chapter 5 . Therefore it is necessary to embrace strategies that allow improving the sensor dependability to satisfy the dependability requirements mentioned above.

This chapter is focussed on proposing an architecture for the AMR sensor system in which the self-X properties and the fault-tolerant method proposed in chapters 4 and 5 are included. In addition, a module for compensating the sensor outputs in real time is included, as well as an IEEE 1687 interface. The dependability assessment of the proposed system has been performed with a focus on the dependability attributes reliability, safety, maintainability and availability.

The outline of the chapter is as follows. Section 6.2 introduces several approaches that can be used to improve the dependability of a system. Section 6.3 explains the architecture of the proposed AMR sensor system. Section 6.4 presents the results of the dependability assessment that has been carried out. Finally, conclusions are drawn in section 6.5.

\subsection{The dependability approach}

As mentioned above, dependability represents the reliance on a system to perform the intended functions under certain conditions during a period of time [Ker10], [Buj04]. Fault-prevention, fault-forecasting, fault-removal, and fault-tolerance are the four 
main means that have been proposed to reinforce the dependability of a system. Fault-prevention aims to prevent the introduction or occurrence of faults in the system in the first place, and therefore focusses mainly on the design and testing phase. Fault-forecasting refers to a set of techniques aimed to estimate the present number of faults, the future incidence, and the likely consequences of faults [Avi04].

Fault-removal is intended for reducing the number of faults present in a system during the development phase as well as in its operational life. In the development phase, verification and diagnosis steps allow detecting a fault to subsequently perform the required corrections to remove it before a system is put into production. On the other hand during the operational life fault-removal is focussed on executing adjustments as required to avoid the occurrence of a failure. The objective is to increase the dependability of the system over the long term by compensating the aging effects. Self-X properties allow a system to perform certain functions on its own, such that it maintains the performance over time [Joh11], [Avi04] .

Fault-tolerance is aimed to preserve the delivery of a correct service in spite of faults. It is usually implemented by redundancy in components, unit or subsystems. However, the overall system then becomes more complex and costly, so a suitable trade-off between the degree of fault-tolerance and the number of redundant components must be found [Ise02], [Avi04].

The dependability of a system should be reinforced since its design phase by trying to clear out all the defects before the system goes into production [Ise02]. However, certain faults as well as the performance degradation due to aging effects cannot be avoided entirely; hence they should be tolerated by additional methods suitable to be used over time. In this thesis, we decided to combine fault-tolerance and self-X properties to improve the dependability of AMR sensors used for angle measurements. A fault-tolerance module allows the system to continue working in case a catastrophic fault occurs. The addition of self-monitoring and self-calibration properties are aimed to keep the sensor performance within its specifications during its lifetime.

\subsection{Architecture}

An architecture for a dependable AMR sensor system is proposed in Figure 6.1. It includes a compensation module to compensate the sensor outputs in real-time for offset voltage as well as amplitude imbalance. The factors used for the compensation are calculated in the performance-monitoring module, and more specifically by selfcalibration in case the self-monitoring sub-module determines that an update of the values is required. The fault-tolerance characteristics allow handling of catastrophic faults in the sensor. The sensor is usually part of a more extensive system, such as a steer-by-wire system, and hence an IEEE 1687 interface is chosen for the sensor system to communicate with other modules that are part of a dependability network implemented in the car. The manager block controls all the activities in the system. The power module defines the power supply that is applied to the AMR sensor. 


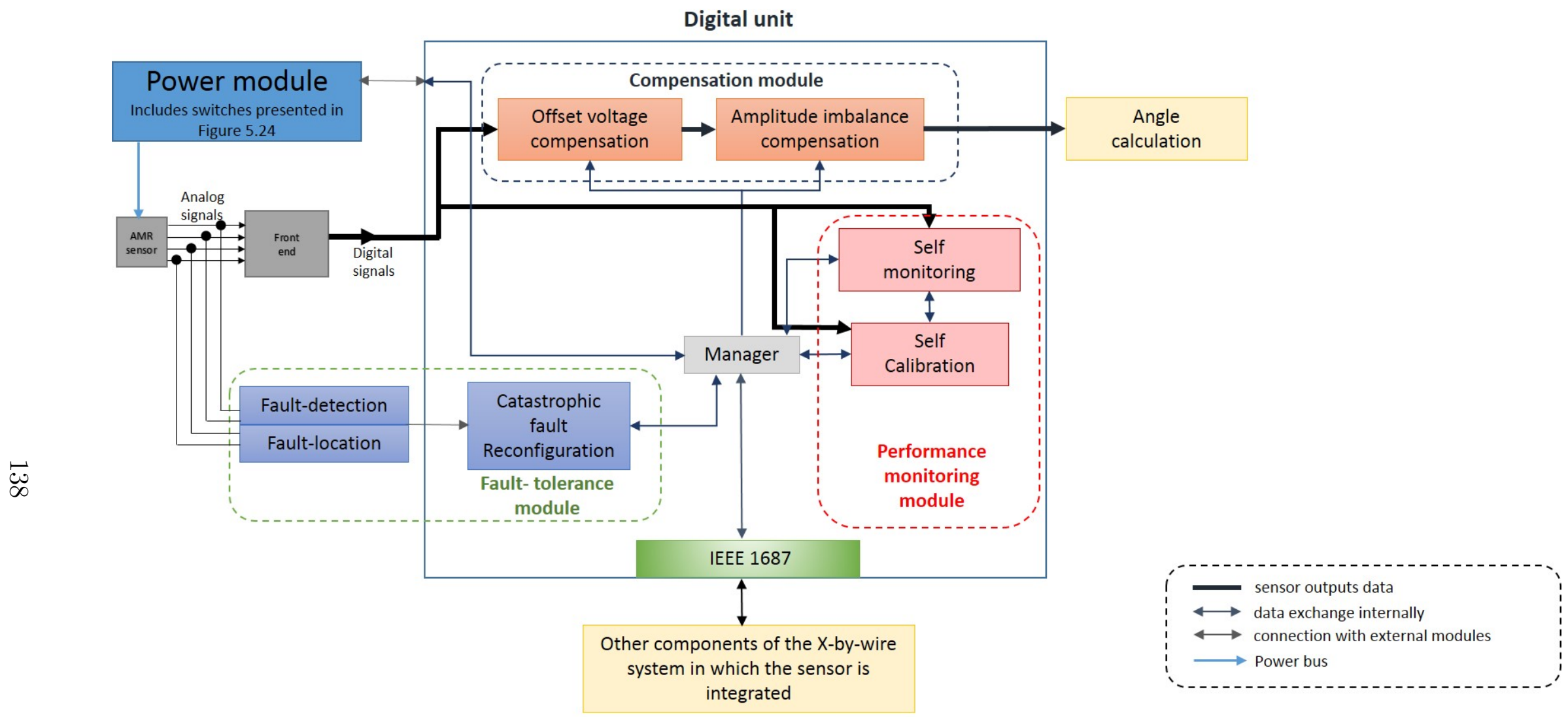

Figure 6.1: Architecture of the proposed AMR sensor system. 
The proposed system is based on digital signal processing, and the operating flow can be summarised as follows. In fault-free condition, the measured sensor outputs are compensated for offset voltage as well as amplitude imbalance and then sent to the module that calculates the magnetic angle. The sensor performance is observed by self-monitoring that verifies the maximum angle error resulting from the offset voltage to determine whether new compensation factors are required or not. The factors are calculated in the self-calibration block and then sent to the compensation module to be applied to the sensor outputs in real time. In case a catastrophic fault occurs, it is detected and localised, and a reconfiguration is proposed such that the sensor can continue its operation. As mentioned before, the manager block (Figure 6.1) is aimed to control all the operations of the system. The IEEE 1687 standard is used to create a dependability network that connects all the internal modules focussed on dependability including the IEEE 1687 interface that allows the connection with other modules that are part of a dependability network implemented in the car.

Next, all the modules are shown in Figure 6.1 are discussed in detail. The digital unit can be implemented either employing a microcontroller or a processor. Although in Figure 6.1 only one digital unit is presented, in practice, it should be considered to have some kind of redundancy to avoid a single point of failure, which also applies for the communication channels and power supply.

\subsubsection{The compensation module}

This module is intended for compensating the sensor outputs for offset voltage and amplitude imbalance in real time. It is connected to the manager from which it receives information regarding the output voltages as well as the compensation factors that should be used in fault-free condition or in the case a catastrophic fault occurs, as detailed in chapter 5 .

\subsubsection{The manager}

The manager coordinates all the functions of the proposed AMR sensor system. In case a catastrophic fault occurs in the AMR sensor, the manager is notified by the fault-tolerance module about the detection of a faulty condition, its location and the reconfiguration must be applied so that the sensor continues its operation. The manager is also responsible for notifying the compensation module about which compensation factors should be used based on the analytical reconfiguration is performed. Regarding performance degradation, the self-monitoring sub-module informs the manager if new compensation factors are needed, which are calculated in the self-calibration sub-module. Then, the manager sends the new factors to the compensation module and stores the information in case it is needed afterwards. The manager is able to connect with the rest of the modules included in the digital unit, as well as with the power module to indicate the configuration of the power supply of the sensor. It can be implemented as a state machine that allows coordinating all the activities of the system. 


\subsubsection{The performance monitoring module}

This module includes the self-X properties proposed in chapter 4 to maintain the sensor performance over time. It consists of a self-monitoring sub-module and a self-calibration sub-module. In the self-monitoring part, the maximum angle error due to the offset voltage is verified as it is the source of angle error that shows the largest drift due to aging effects, as detailed in chapter 3. The maximum angle error resulting from the offset voltage is calculated with Equation (4.9), presented in chapter 4 . In case, the value is larger than the value is allowed, the system manager is informed that new compensation factors are required. The maximum angle error is permitted is determined by the application in which the sensor is used.

Figure 6.2 shows the maximum angle error due to offset voltage in one of the AMR sensors used in the second aging test is detailed in chapter 3. The original angle has been calculated by subtracting the angle calculated before and after compensating the sensor outputs for offset voltage. The other angle has been obtained by applying Equation (4.9), included in the self-monitoring sub-module. The average difference between these two angles is $0.04^{\circ}$, which means that self-monitoring provides an accurate value of the maximum angle error due to the offset voltage.

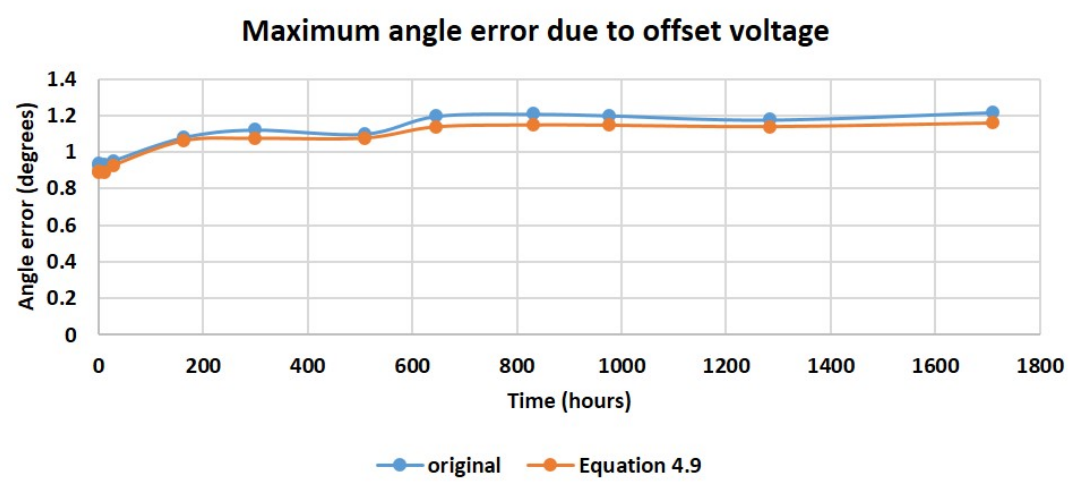

Figure 6.2: Maximum angle error due to offset voltage in one of the AMR sensors used in the second aging test as detailed in chapter 3 .

The self-calibration sub-module allows calculating the compensation factors for the offset voltage as well as amplitude imbalance online using the compensation method proposed in chapter 4 . Online means that the compensation factors are calculated without disturbing the normal operation of the sensor, as it is assumed that the sensor is used in applications in which complete cycles of the sinusoidal signals at the sensor outputs are obtained. The compensation method allows calculating first the compensation factors for voltage and then for amplitude imbalance.

Figure 6.3 shows the variation of the maximum angle error in a sensor used in the second aging test detailed in chapter 3 , of which the maximum angle error due to the offset voltage is shown in Figure 6.2. Three scenarios have been considered including no compensation (without compensation), and compensating for offset 
voltage and amplitude imbalance using factors calculated at the start of the sensor life (calibration during manufacturing); finally compensating for offset voltage and amplitude imbalance using the proposed self-calibration module to update the compensation factors over time (our compensation method during lifetime). In the first two scenarios, the angle error drifts approximately $0.3^{\circ}$, while in the third one it is fairly constant over time showing drifts of approximately $0.02^{\circ}$. The remaining angle error after compensation is mainly because of measurement inaccuracies in the set-up used to measure the voltages at the sensor outputs.

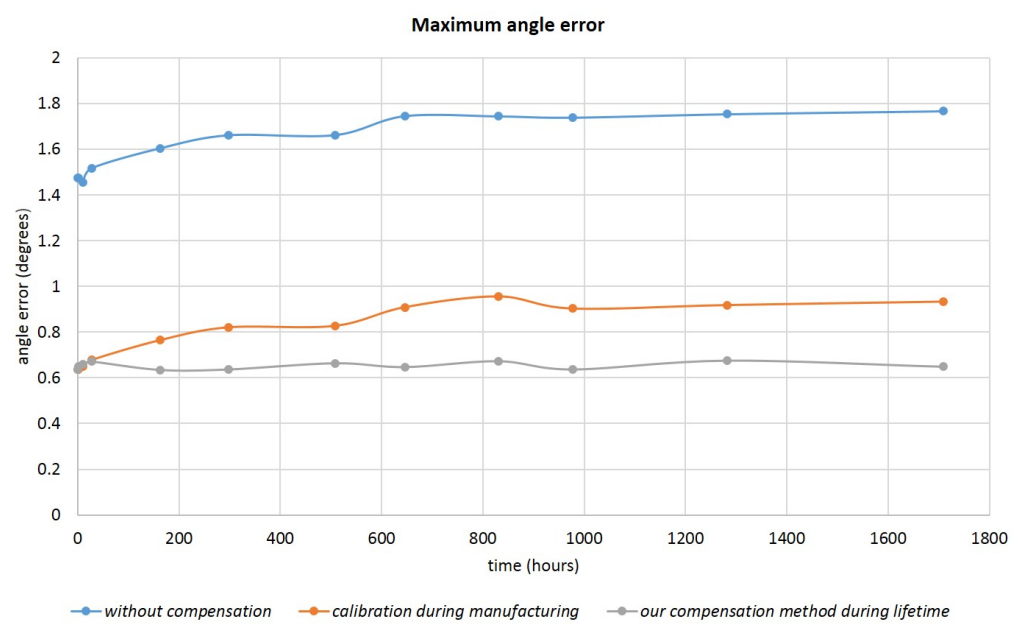

Figure 6.3: The maximum angle error in an AMR sensor considering three scenarios: no compensation (without compensation), compensating with factors calculated at the start of the sensor life (calibration during manufacturing), compensating with factors obtained with the self-calibration sub-module (our compensation method during lifetime).

\subsubsection{The fault-tolerance module}

This module represents the fault-tolerant system proposed in chapter 5. It consists of three sub-modules to detect, localise, and recover the sensor operation in case a catastrophic fault occurs in the bridge resistances or the connections to the power supply or ground of an AMR sensor. Table 6.1 summarises the fault conditions, the voltages used to determine their location, and the applicable reconfiguration depending on the type of fault present.

The three sub-modules can be implemented in the digital unit; however, this affects the downtime of the system in faulty conditions. As mentioned above, although the maximum downtime allowed is determined by the application, a zero or short downtime is always desired, especially in safety-critical applications. Therefore, we propose to execute fault-detection and fault-location in the analogue domain by implementing, for example, comparators aimed for detecting abnormal voltages at the sensor outputs. The goal is to reduce the time required to recover the system operation. 
Table 6.1: Fault-tolerant system for catastrophic faults in AMR sensors

\begin{tabular}{|c|c|c|c|c|c|}
\hline \multirow[t]{2}{*}{ Fault Condition } & \multicolumn{4}{|c|}{ Fault Detection } & \multirow{2}{*}{$\begin{array}{c}\text { Fault Recovery } \\
\text { (Voutbridge1) (Voutbridge2) }\end{array}$} \\
\hline & vop1 & von1 & von2 & vop2 & \\
\hline Fault Free & $\mathrm{VCC} / 2 \pm \mathrm{AMR}$ & $\mathrm{VCC} / 2 \pm \mathrm{AMR}$ & $\mathrm{VCC} / 2 \pm \mathrm{AMR}$ & $\mathrm{VCC} / 2 \pm \mathrm{AMR}$ & (vop1-von1) (vop2-von2) \\
\hline R2(open) or R1(short) & $\approx \mathrm{VCC}$ & $\mathrm{VCC} / 2 \pm \mathrm{AMR}$ & $\mathrm{VCC} / 2 \pm \mathrm{AMR}$ & $\mathrm{VCC} / 2 \pm \mathrm{AMR}$ & (vop2-von1) (von2-von1) \\
\hline R3(short) or R4(open) & $\mathrm{VCC} / 2 \pm \mathrm{AMR}$ & $\approx 0$ & $\mathrm{VCC} / 2 \pm \mathrm{AMR}$ & $\mathrm{VCC} / 2 \pm \mathrm{AMR}$ & (vop1-von2) (vop1-vop2) \\
\hline R23(open) or R24(short) & $\mathrm{VCC} / 2 \pm \mathrm{AMR}$ & $\mathrm{VCC} / 2 \pm \mathrm{AMR}$ & $\approx \mathrm{VCC}$ & $\mathrm{VCC} / 2 \pm \mathrm{AMR}$ & (vop2-von1) (vop1-vop2) \\
\hline R22(short) or R21(open) & $\mathrm{VCC} / 2 \pm \mathrm{AMR}$ & $\mathrm{VCC} / 2 \pm \mathrm{AMR}$ & $\mathrm{VCC} / 2 \pm \mathrm{AMR}$ & $\approx 0$ & (vop1-von2) (von2-von1) \\
\hline R22(open) or R21(short) & $\mathrm{VCC} / 2 \pm \mathrm{AMR}$ & $\mathrm{VCC} / 2 \pm \mathrm{AMR}$ & $\mathrm{VCC} / 2 \pm \mathrm{AMR}$ & $\approx \mathrm{VCC}$ & (vop1-von2) (von2-von1) \\
\hline $\begin{array}{c}\text { Broken bridge } 1 \\
\text { supply connection }\end{array}$ & 0 & 0 & $\mathrm{VCC} / 2 \pm \mathrm{AMR}$ & $\mathrm{VCC} / 2 \pm \mathrm{AMR}$ & switch power supply \\
\hline $\begin{array}{c}\text { Broken bridge } 2 \\
\text { supply connection }\end{array}$ & $\mathrm{VCC} / 2 \pm \mathrm{AMR}$ & $\mathrm{VCC} / 2 \pm \mathrm{AMR}$ & 0 & 0 & switch power supply \\
\hline $\begin{array}{l}\text { Broken bridge } 2 \\
\text { ground connection }\end{array}$ & $\mathrm{VCC} / 2 \pm \mathrm{AMR}$ & $\mathrm{VCC} / 2 \pm \mathrm{AMR}$ & $\mathrm{VCC}$ & $\mathrm{VCC}$ & switch power supply \\
\hline
\end{tabular}

The information is linked with Figure 4.7. AMR represents the voltage due to magnetoresistance effect and VCC the voltage of the power supply. 
Once a faulty condition is detected and localised based on the abnormal voltages present at the affected half-bridge of half-bridges, the manager is notified so the faulty condition can be handled immediately. This means that based on the information received, the reconfiguration sub-module specifies the output voltages that should be used to calculate the magnetic angles. This information is sent to the manager, which first determines the new compensation factors and then sends them all to the compensation module. As the offset voltage per each half-bridge is known, it is possible to use updated values for compensation.

Figure 6.4 shows the calculated angles using the output voltages of a commercial AMR sensor in standard configuration as well as with the proposed reconfigurations in fault-recovery in case of a catastrophic fault in any of the bridge resistances of the sensor. As explained in chapter 5 , the difference of the calculated angles between the set magnetic angles $100^{\circ}$ to $120^{\circ}$ is resulting from the $45^{\circ}$ phase difference of the output voltages defined in fault-recovery. However, this phase difference is known in advance, so it can be compensated and the magnetic angle can still be calculated despite a faulty resistance in the sensor. In case of a broken connection either to the power supply or ground in one bridge, the standard sensor outputs are still used to calculate the angles, but it is required to switch the connection to the other available power supply.

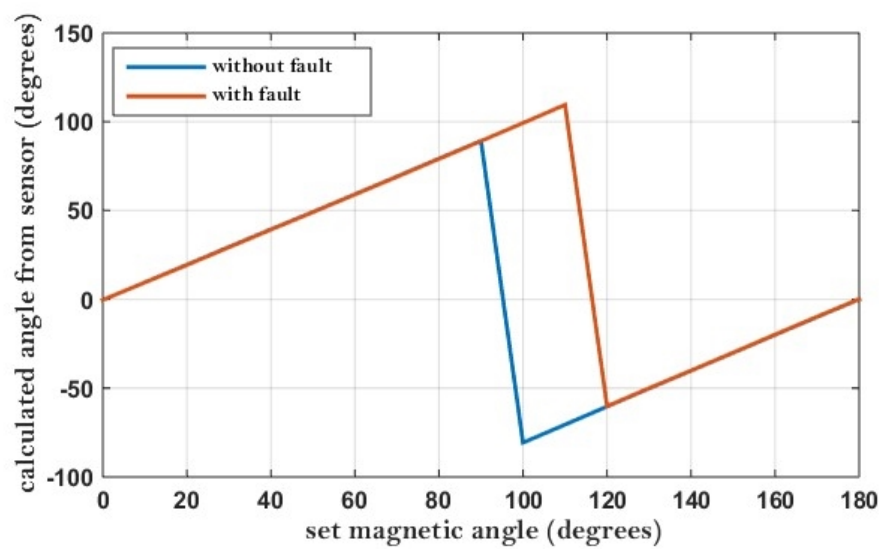

Figure 6.4: Calculated angle with and without faulty conditions in the bridge resistances of the AMR sensor.

\subsubsection{The IEEE 1687 module}

The IEEE 1687 (IJTAG) is a standard aimed to provide a standardised methodology for accessing and operating embedded instruments [Shi14]. It allows creating an instrument access infrastructure (network) with reconfigurable scan chains, in which the reconfiguration is done by incorporating programmable components into the network structure. One such programmable component is the Segment Insertion Bit (SIB) that permits including or excluding scan segments from the active scan-path. It can be used for bypassing a segment of a network, which can be simply one 
instrument or a sub-network consisting of several instruments grouped forming a cluster. Therefore, it is possible to create a hierarchical network that consists of modules (instruments, SIB) configured with IJTAG host-client relationships. A client interface of a module is attached to the host interface of another module in the network in a higher hierarchical level [Ibr18], [IEE14], [ASS11]. Figure 6.5 shows an example of a hierarchical IJTAG network with three levels in which level 1 (L1) includes all the instruments of the network.

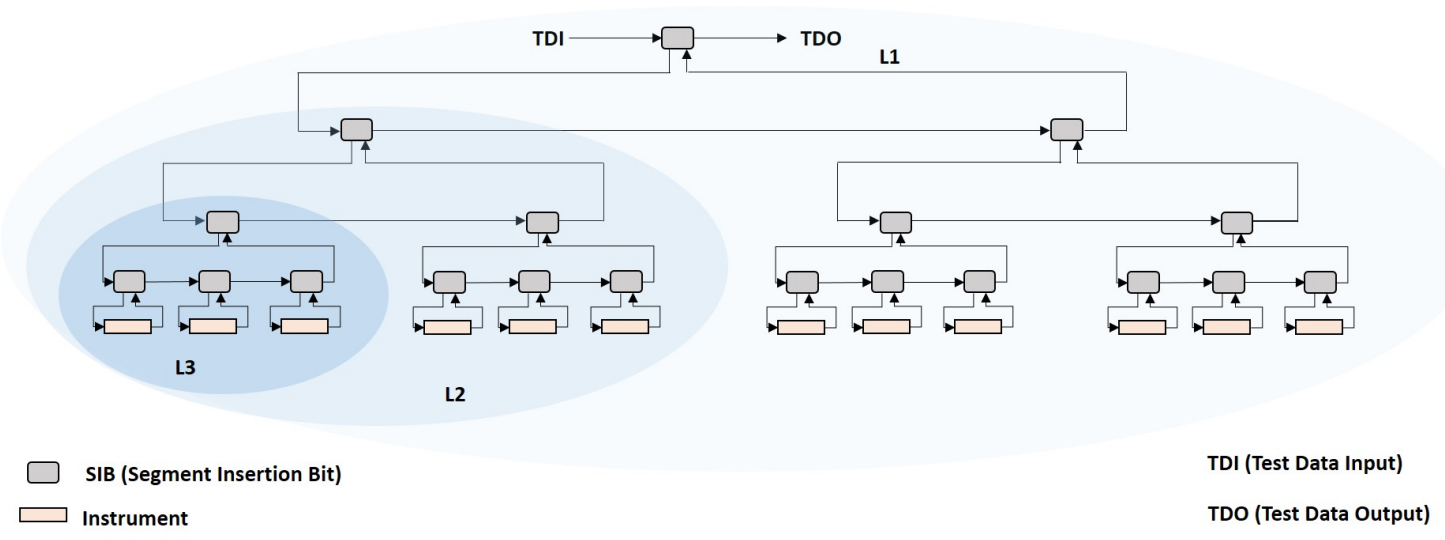

Figure 6.5: Example of a hierarchical IJTAG network [Ibr18].

IJTAG can also be used for dependability management purposes in systems that include embedded instruments aimed to enhance the dependability. These parts are referred to as dependability instruments, which are handled by a dependability manager [Ibr18]. An efficient and regular network for system health-monitoring and fault-management can be created as proposed by Jutman [Jut13]. Fault-management describes the actions that must be carried out to maintain the system operation in case of fault conditions, which includes fault-detection, fault-diagnosis, faultevaluation and decisions on actions.

In our AMR sensor system, it is possible to use the IEEE 1687 standard for dependability management [Ibr18] considering the manager module as the dependability manager (DM), while the performance monitoring module, fault-tolerant module, compensation module and power module can be regarded as dependability instruments. This is because all these modules are aimed at improving the dependability of the AMR sensor. Additionally, the IEEE 1687 interface allows the system to connect with other modules that are part of a dependability network implemented in the car. Hence, it is possible to inform if a fault occurred, its type and whether it was corrected or not, but also transmit information regarding the performance of the sensor. In case a catastrophic fault occurs it is possible to inform the car driver that a repair is required and the car can continue in operation for a limited period of time. 
Figure 6.6 shows the IJTAG network of the AMR sensor system in which the instruments are accessed throughout a scan path and SIBs. The SIBs are connected to TDRs (Test Data Register) that act as a read/write front-end to the instruments. It is considered that this network is part of a hierarchical IJTAG network in which all the instruments of the AMR sensor system form a cluster that is controlled by a cluster dependability manager (CDM). A cluster SIB (CSIB1) enables the access to the cluster, while the dependability manager SIB (DSIB1) is aimed to access the DM TDRs. By the control MUX it is taken care of whether the CDM or a higher-level controller (via CSIB) accesses to the instruments. The IJTAG client interface [Ibr18] allows the connection to the host interface of another module in the network at a higher hierarchical level.

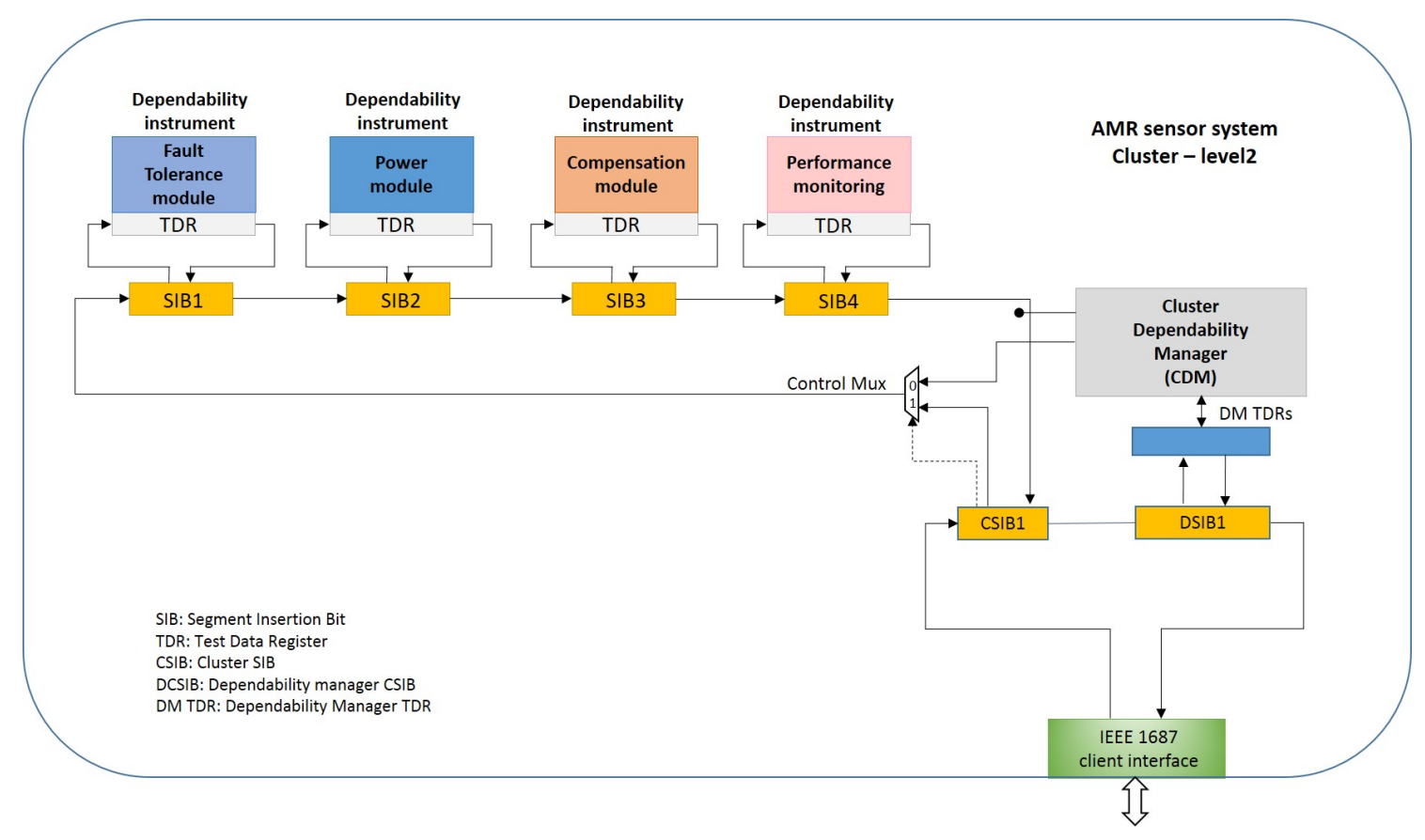

Figure 6.6: IJTAG network for dependability management in our AMR sensor system.

Figure 6.7 shows the IJTAG network of an X-by-wire system which consists of several clusters including the cluster of our AMR sensor system (L2) shown in Figure 6.6. All of them are considered to form a top-level cluster (L1) accessed by a cluster SIB (CSIB0) and controlled by a system dependability manager (SDM). The interface IEEE 1149.1 allows the access to the instruments for several purposes such as test, debug, diagnosis, etc. 


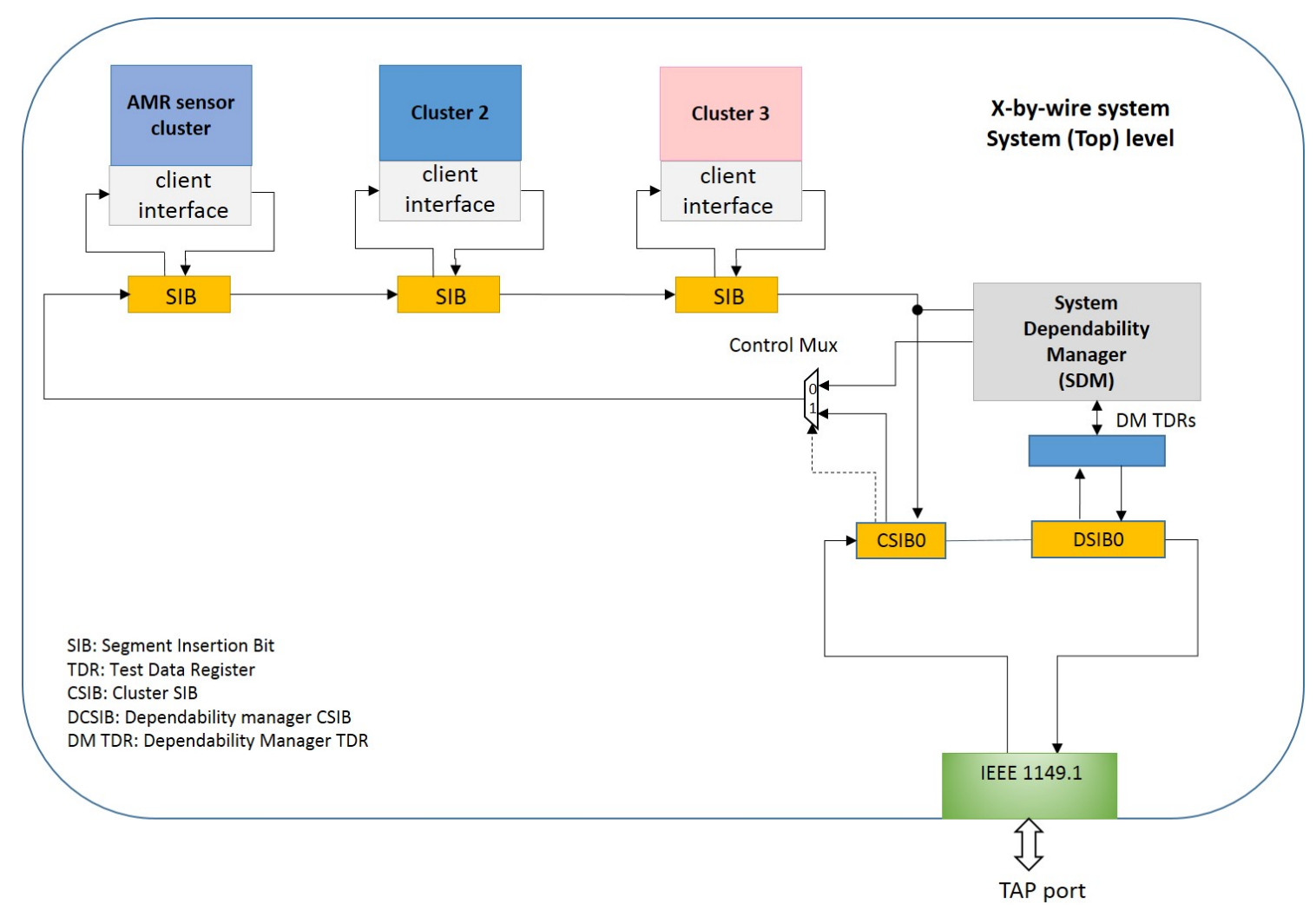

Figure 6.7: Hierarchical IJTAG network for dependability management on an X-by-wire system.

By default, the IEEE 1687 standard considers a master/slave communication in which the dependability manager, as master, always starts the communication with the dependability instruments defined as slaves [Ibr18]. However, by the usage of a methodology as presented in [Jut13] for instrument interrupt, it is possible to enable an instrument-initiated communication session with the dependability manager, which is useful in case of catastrophic faults and performance degradation. Two types of interrupts can be used as defined by Ibrahim in [Ibr18]. Preemtive Interrupt (PI) indicates the occurrence of a critical event that requires immediate attention, for example a catastrophic fault. Non-Preemtive interrupt (NPI), refers to a request for initiating a communication session from one of the instruments, but not immediate attention is required, for example if the self-monitoring sub-module determines that new compensation factors are needed.

Overall, based on the digital signal processing, our AMR sensor system allows handling performance degradation as well as catastrophic faults in AMR sensors used in automotive applications. Although the compensation factors cannot be calculated in real-time, this is also not necessary because the considered undesired parameters drift slowly over time. As processing resources are limited during the sensor lifetime, it is better to compensate the sensor outputs in real-time but calculate the compensation factors only if it is actually required. Taking into consideration 
the drifting trends of the undesired parameters included in the sensor outputs shown in chapter 3 , in practice it is feasible to just update the factors for offset voltage. In case of a catastrophic fault in the bridge resistances, the compensation factors cannot be longer updated, but the sensor can continue its operation and the car driver has time to take the car to the garage. The detection and localisation of catastrophic faults are executed in the analog domain to reduce the downtime of the system before it can recover its operation, which is especially important in safety-critical applications. The IEEE 1687 standard is considered for the connection of all the system modules aimed to improve the dependability of the AMR sensor, as well as for the communication with other modules that are part of a network for dependability and/or fault-management.

\subsection{Dependability assessment}

\subsubsection{Introduction}

The international standard IEC 60300-3 recommends a set of analysis techniques for dependability assessment of electronic systems, as shown in Table 6.2 [IEC03]. These can be used for quantitative or qualitative analysis, with the qualitative analysis mainly focussed on the determination of fault modes, failure mechanisms, causes, effects and consequences of failures, as well as possible repair/recovery strategies. On the other hand, quantitative analysis aims at defining numerical reference data to be used as input parameters of reliability/availability models, but also to assess the improvement of the dependability of a system as a result of a change in design or manufacturing [Ber13].

The standard IEC 60300-3 proposes some guidelines for choosing a technique, but there is not a general rule for selecting the best method to assess the dependability of a specific system. In our research, Markov analysis has been used to evaluate the dependability improvement that can be achieved by implementing our proposed AMR sensor system. The Markov analysis is a stochastic technique for modelling the state transitions of a system in which its future state only depends on the current state [IEC03], [Ber13]. This technique is a proper tool for modelling complex systems involving repair, redundancy and fault-tolerance, so it is widely used to quantify system dependability in terms of performance, availability, reliability, maintainability and safety [Fuq03], [Son16]. 
Table 6.2: Dependability analysis techniques IEC-60300-1-3 [IEC03]

\begin{tabular}{|c|c|c|}
\hline Technique & Qualitative analysis & Quantitative analysis \\
\hline Fault Tree Analysis & Fault combinations & $\begin{array}{l}\text { Calculation of system } \\
\text { reliability, availability }\end{array}$ \\
\hline Event tree analysis & Failure sequences & $\begin{array}{c}\text { Calculation of system } \\
\text { failure rates }\end{array}$ \\
\hline $\begin{array}{l}\text { Reliability block } \\
\text { diagram analysis }\end{array}$ & Success paths & $\begin{array}{l}\text { Calculation of system } \\
\text { reliability, availability }\end{array}$ \\
\hline Markov analysis & Failure sequences & $\begin{array}{l}\text { Calculation of system } \\
\text { reliability, availability }\end{array}$ \\
\hline Petri net analysis & Failure sequences & $\begin{array}{l}\text { provide the system } \\
\text { description for Markov }\end{array}$ \\
\hline $\begin{array}{l}\text { Failure modes and } \\
\text { effects analysis }\end{array}$ & Effect of failures (FMEA) & $\begin{array}{c}\text { Calculation of system } \\
\text { failure rates }\end{array}$ \\
\hline
\end{tabular}

The Markov model of a given system consists of a state transition diagram that includes the possible states and the possible transition paths between these states, as shown in Figure 6.8 for a system with two states $(S 1, S 0)$. A common practice used for reliability modeling is to describe the probability of transitioning from state to state with a transition rate related to the elapsed time before the system moves from the originating state to the target of the transition. For electronic components, this transition rate is usually represented by the failure rate $(\lambda)$ of the components, which are assumed to fail according to an exponential distribution that results in a constant failure rate during their useful life. The system can be in only one state at a time, and from time to time it may transition from one state to another following one of the set of inter-state transitions. A state can be a non-failed state (represented below by a white circle) in which the system is operating correctly (either with full functionality or in some type of degraded mode); or a failed state that refers to a non functional system (represented below by a grey circle) [Fuq03], [Kim02].

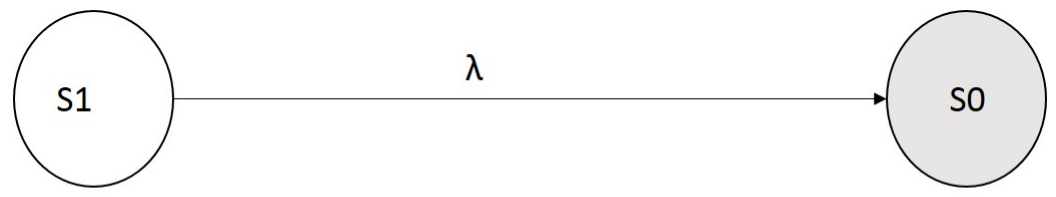

$\lambda$ : failure rate

Figure 6.8: Markov model of a system with a non-failed state (S1) and a failed state $(\mathrm{S} 0)$. 
The process of solving a Markov model by a state-space approach produces as output the probabilities of being in each of the states at a specific time $(t)$. The Markov model states can be described by differential equations that can be represented with a vector notation as shown in Equation (6.1) [Kha14].

$$
P^{\prime}(t)=P(t) * A
$$

where $P(t)$ is a $1 \times n$ row vector that refers to the probability of being in an each state at time $t$, and $A$ is a $n \times n$ transition matrix with $n$ equal to the number of states in the system. The solution of Equation (6.1) can be written as in Equation (6.2) and represents the probability of the states:

$$
P(t)=P(0) *\left[e^{A * t}\right]
$$

where $e^{A * t}$ is a $1 \times n$ vector and $P(0)$ is a $1 \times n$ initial probability row vector that includes the initial state of the system. The sum of the probabilities over non-failed states is defined as the system reliability, as shown in Equation (6.3).

$$
\operatorname{Reliability}(t)=\sum_{i} P_{i}(t)
$$

where $P_{i}$ represents the probability of the non-failed states of the system.

Equations (6.1), (6.2) and (6.3) are further used in the next section for dependability assessment purposes.

\subsubsection{Dependability assessment of the proposed AMR sen- sor system}

The Markov analysis technique has been used to perform a dependability assessment focussed on the dependability attributes reliability, safety, maintainability and availability. For comparison purposes, besides our AMR sensor system, a single AMR sensor, Dual Modular Redundancy (DMR) and Triple Modular Redundancy (TMR) have also been considered in the assessment. The schemes of these systems are shown in Figure 6.9. All of them except the single AMR sensor use redundancy to guarantee that the system can continue its operation in case a catastrophic fault occurs. An important concept in a redundant system is coverage $(c)$, which means the probability of reconfiguring a system successfully if a fault occurs [Pen12]. Therefore we have included this parameter in the Markov models of our system as well for the systems with DMR and TMR. Regarding the failure rate $(\lambda)$, an AMR sensor consists of 8 magnetoresistances configured into two Wheatstone bridges, as detailed in chapter 2. Taking into account that the sensor fails if one of the resistances fail, the same failure rate can be assumed for the magnetoresistance elements and the sensor. In the following, the Markov models of the different systems are presented. 


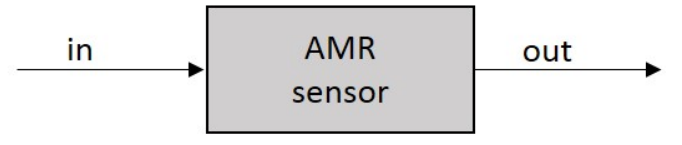

a. Single AMR sensor

范

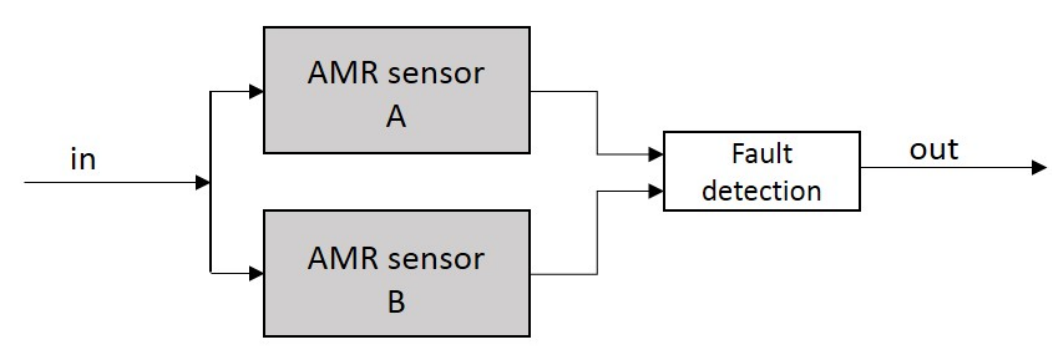

b. Dual Modular Redundancy (DMR)

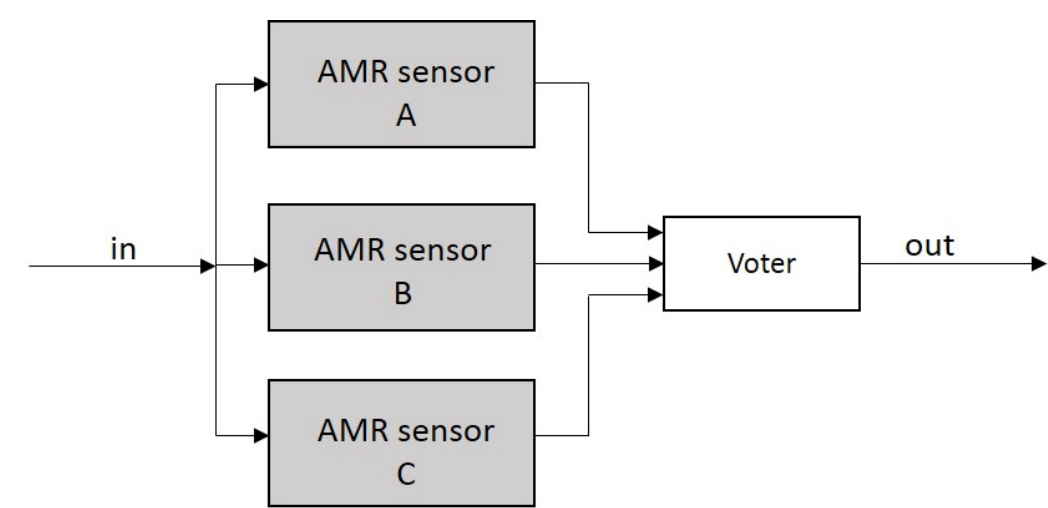

c. Triple Modular Redundancy (TMR)

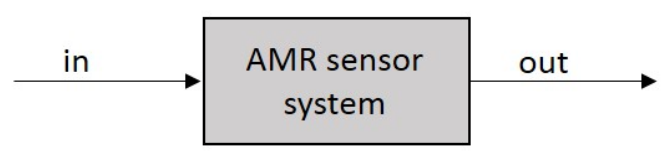

d. Our AMR sensor system

An AMR sensor for angle measurements consists of eight magnetoresistances configured into two Wheatstone bridges positioned at an angle of $45^{\circ}$ with respect to each other.

Figure 6.9: Different AMR sensor schemes. 
Single AMR sensor: this refers to only one AMR sensor as shown in Figure 6.9a. Because an AMR sensor is not considered redundant by default, its Markov model can be described as shown in Figure 6.8. The sensor starts in S1 at 0 hours and it moves to $\mathrm{S} 0$ once a fault condition occurs. The state transition matrix $A_{A M R}$ for this system is shown in Equation (6.4) and the initial state conditions $P[0]_{A M R}$ in Equation (6.5) in which is specified that the system starts in the non-failed state $S 1$. From these equations and Equations (6.2) and (6.3), the reliability of the system is derived which is presented in Equation (6.6) [Smi11].

$$
\begin{gathered}
A_{A M R}=\left[\begin{array}{cc}
-\lambda & 0 \\
0 & \lambda
\end{array}\right] \\
P[0]_{A M R}=\left[P_{1}(0) \quad P_{0}(0)\right]=\left[\begin{array}{ll}
1 & 0
\end{array}\right] \\
\text { Reliability }{ }_{A M R}(t)=P_{1}(t)=e^{-\lambda * t}
\end{gathered}
$$

System with sensors in Dual Modular Redundancy (DMR) approach: this includes two AMR sensors as well as a module for fault-detection and reconfiguration as shown in Figure 6.9b. The sensors are in hot standby configuration with dynamic redundancy that has been explained in chapter 2. The system remains functional as long as one of the two sensors is in operation.

The Markov model of the sensors is presented in Figure 6.10. In this case, taking into account that the sensors have the same failure probability, a reduced Markov model has been considered in which some states are combined [Fuq03]. The system starts fully operational (both sensors functional) in state $S 2$, then it moves to state $S 1$ if one sensor fails, but the faulty condition is correctly handled, so the system can continue in operation with the other sensor (successful fault-detection and faultreconfiguration assumed). Otherwise, it moves to the failed state SO. From state $S 1$, the system goes to state $S 0$ once the remaining sensor fails as well. The probability of transitioning from the state $S 2$ to $S 1$ or $S 0$ includes $2^{*} \lambda$ because there are two functional AMR sensors that can fail, while from the state $S 1$ to $S 0$ only one sensor is in operation. The coverage factor $c$ is defined as the probability that the system is able to handle a fault condition while it is in state $S 2$. 


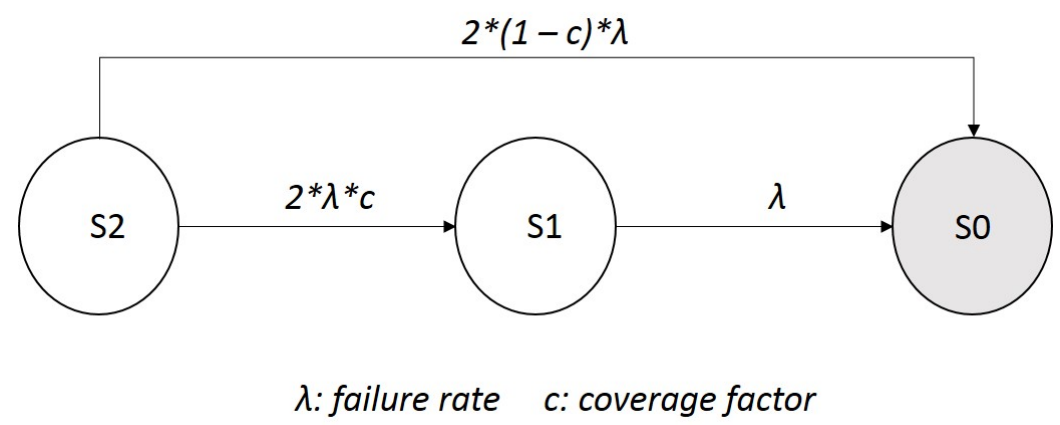

Figure 6.10: Reduced Markov model of the sensors with Dual Modular Redundancy (DRM) in which S2 and $S 1$ are non-failed states while $S 0$ is a failed state.

Equation (6.7) shows the transitioning matrix $A_{2 A M R}$ that corresponds to the Markov model shown in Figure 6.10. The initial state conditions are as presented in Equation (6.8) because the system starts fully operational in state S2.

$$
\begin{gathered}
A_{2 A M R}=\left[\begin{array}{ccc}
-2 * \lambda & 2 * \lambda * c & 2 * \lambda *(1-c) \\
0 & -\lambda & \lambda \\
0 & 0 & 0
\end{array}\right] \\
P[0]_{2 A M R}=\left[\begin{array}{lll}
P_{2}(0) & P_{1}(0) & P_{0}(0)
\end{array}\right]=\left[\begin{array}{lll}
1 & 0 & 0
\end{array}\right]
\end{gathered}
$$

The probabilities of the sensors being in a state at time $t$ are calculated using the Equations (6.2), (6.7) and (6.8). Then, based on Equation (6.3) it can be derived that the reliability of the sensors configured in the DMR approach is written by Equation (6.9):

$$
\text { Reliability }_{2 A M R}(t)=P_{2}(t)+P_{1}(t)=2 * c * e^{-\lambda * t}+(1-2 * c) * e^{-2 * \lambda * t}
$$

In order to obtain the reliability of the complete system, the reliability of the module for fault-detection and reconfiguration $\left(R_{d}\right)$ should be included as shown in Equation (6.10).

$$
\text { Reliability }_{\text {systemwithDMR }}=\text { Reliability }_{2 A M R} * R_{d}
$$

System with sensors in Triple Modular Redundancy (TMR) set-up: this system includes 3 AMR sensors and a voter module, as shown in Figure 6.9c. In this case, the system is considered functional as long as at least 2 out of the 3 AMR sensors are operational. A TMR system is usually aimed to handle only the first failure, while it does not operate after two failures. The features required to make it work 


\subsection{Dependability assessment}

after two failures may increase its complexity such that it degrades the ability to survive the first failure [Ham03]. This type of system is more often used because of its excellent coverage factor $(c)$ of the first failure, rather than because it can operate after two failures.

Figure 6.11 shows the reduced Markov model of the AMR sensors with TMR. As it was done for the system with DMR, in this case it is also considered that the sensors have the same failure probability, so a reduced Markov model has been used [Fuq03]. The model starts in S2 with 3 functional AMR sensors. At the moment that one of the sensors fails, the system moves to state $S 1$ if the faulty condition is correctly handled or to the failed state $S 0$ in case the system cannot continue in operation. As explained for the DMR system, the coverage factor $c$ represents the probability that the system can handle successfully a faulty condition in state S2. The system goes from state $S 1$ to $S 0$ in the case that only one AMR sensor is functional. The probabilities of transitioning from state $S 2$ and state $S 1$ are multiplied by 3 and 2 respectively due to the amount of functional sensors at these states.

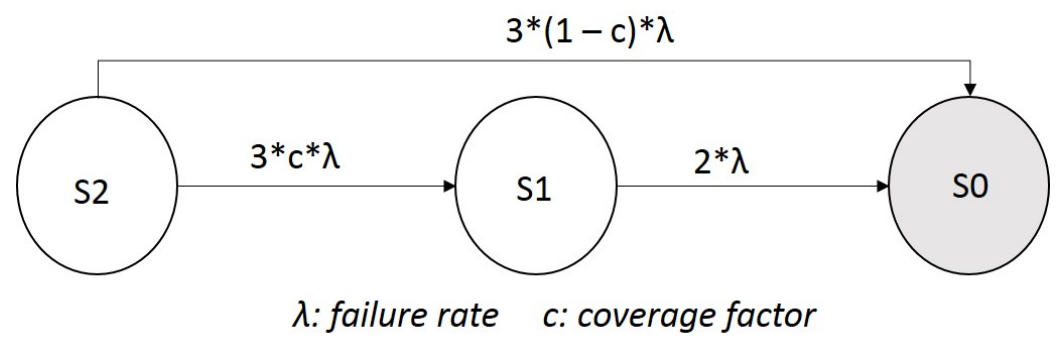

Figure 6.11: Reduced Markov model of the sensors configured with Triple Modular Redundancy (TRM) in which $S 2$ and $S 1$ are non-failed states while $S 0$ is a failed state.

Equation (6.11) presents the state transition matrix $\left(A_{3 A M R}\right)$ that corresponds to the sensors configured with TMR. The initial state conditions are equal to the conditions presented in Equation (6.8) for the system with DMR. Combining the last two equations and Equation (6.2) the probabilities of the states of the Markov model shown in Figure 6.11 are calculated in order to determine the reliability regarding the sensors based on Equation (6.3), which is given by Equation (6.12).

$$
A_{3 A M R}=\left[\begin{array}{ccc}
-3 * \lambda & 3 \lambda c & 3 * \lambda(1-c) \\
0 & -2 \lambda & 2 \lambda \\
0 & 0 & 0
\end{array}\right]
$$

$$
\text { Reliability }_{3 A M R}(t)=P_{2}(t)+P_{1}(t)=3 * c * e^{-2 * \lambda * t}+(1-3 * c) * e^{-3 * \lambda * t}
$$

To calculate the reliability of the system with sensors in TMR configuration Equation (6.12) should be multiplied by the reliability of the voter module $\left(R_{v}\right)$ as indicated 
in Equation (6.13).

$$
\text { Reliability }_{\text {systemwithTMR }}=\text { Reliability }_{3 A M R} * R_{v}
$$

Our AMR sensor system: this system has been detailed in section 6.3. The Markov model of the system is shown in Figure 6.12 in which it starts fully functional in state S2. From state $S 2$ it moves to state $S 1$ if a faulty condition is successfully handled so the sensor can continue in operation (successful fault-reconfiguration), otherwise, it goes to state $S 0$. The system moves from state $S 1$ to state $S O$ in the case it is not functional anymore. As mentioned in the previous systems, the coverage factor $c$ denotes the probability that the system is capable of transitioning from state $S 2$ to $S 1$. Compared to the DMR and TMR systems, the probabilities of transitioning between states only includes one $\lambda$ because there is only one AMR sensor. Instead of using physical redundancy as in DMR and TRM, our system applies analytical redundancy to guarantee the sensor continues in operation despite the occurrence of a catastrophic fault in any of its magnetoresistances. An important remark is that the failure rate in the Markov model of our system includes the failure rate of the AMR sensor itself and the digital unit as well.

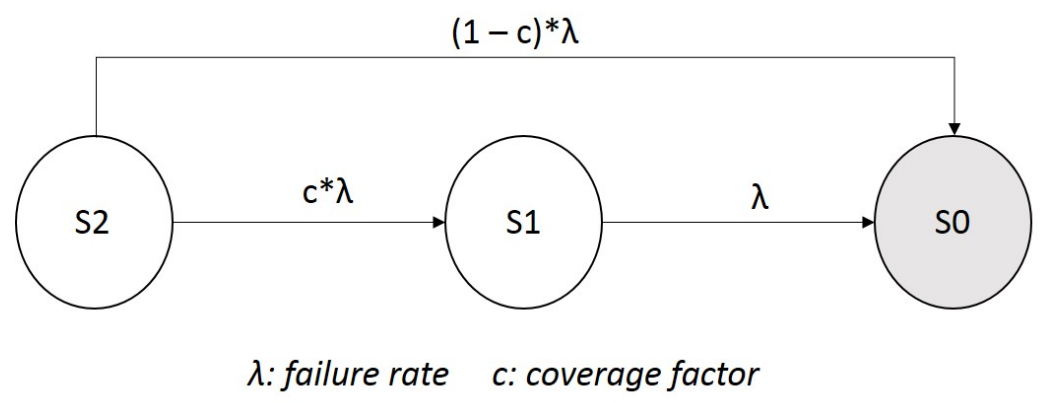

Figure 6.12: Markov model of our system in which $S 2$ and $S 1$ are non-failed states while $S 0$ is a failed state.

Equation (6.14) shows the transitioning matrix $\left(A_{A M R+}\right)$ for this system, while the initial state conditions are as shown in Equation (6.8) for the DRM system. From these equations and Equation (6.2), the probabilities of the states are obtained and subsequently the system reliability based on Equation (6.3). The system reliability is described by Equation (6.15).

$$
\begin{gathered}
A_{A M R+}=\left[\begin{array}{ccc}
-\lambda & c * \lambda & (1-c) * \lambda \\
0 & -\lambda & \lambda \\
0 & 0 & 0
\end{array}\right] \\
\text { Reliability }_{A M R+}(t)=P_{2}(t)+P_{1}(t)=e^{-\lambda * t}+(c * \lambda * t) * e^{-\lambda * t}
\end{gathered}
$$


Equation (6.15) has been obtained considering that the failure rate $(\lambda)$ is the same after performing the reconfiguration is required for the sensor to continue its operation. However, one can also consider that the failure rate has increased for the reconfigured system, in which case the Markov model is as depicted in Figure 6.13. The failure rate between the states $S 1$ and $S 0$ is now $\lambda 1$ instead of $\lambda$ as in Figure 6.12. Note that the following holds if $\lambda \neq \lambda 1$.

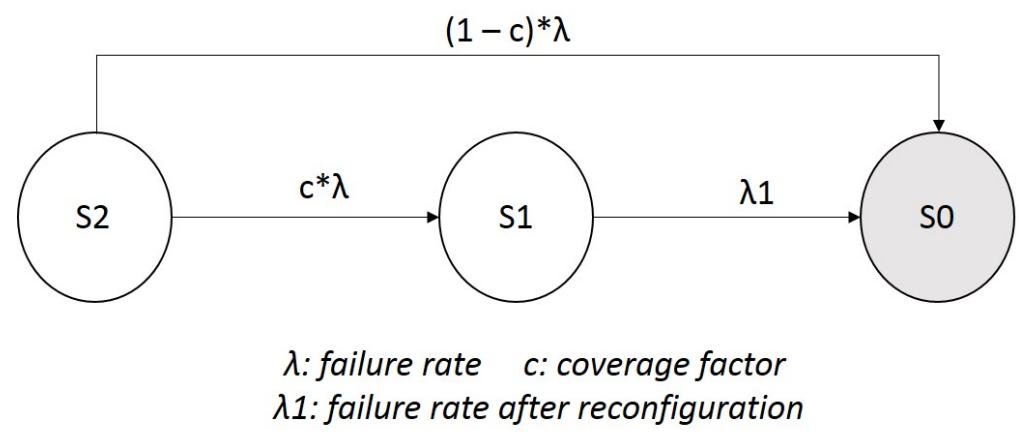

Figure 6.13: Markov model of our system in which $S 2$ and $S 1$ are non-failed states while $S 0$ is a failed state.

The transitioning matrix that corresponds to the Markov model presented in Figure 6.13 is presented in Equation (6.16), while the resulting reliability is described by Equation (6.17). This equation is expected to be better adjusted to a real-life scenario.

$$
A_{A M R+a}=\left[\begin{array}{ccc}
-\lambda & c * \lambda & (1-c) * \lambda \\
0 & -\lambda 1 & \lambda 1 \\
0 & 0 & 0
\end{array}\right]
$$

Reliability $_{A M R+a}(t)=P_{2}(t)+P_{1}(t)=e^{-\lambda * t}+\frac{c * \lambda}{(\lambda 1-\lambda)} * e^{-\lambda * t}-\frac{c * \lambda}{(\lambda 1-\lambda)} * e^{-\lambda 1 * t}$

In order to verify the reliability performance of the four systems presented above, the following parameter values have been used. These have been defined taking into consideration the values often considered for typical automotive electronic components [Ham03]. The failure rate $\lambda$ used for the AMR sensor, the system with DMR and the system with TMR is $1.10^{-7} / \mathrm{hr}$ that corresponds to the failure rate of the sensor. In the case of our system, $\lambda$ is defined as $6.10^{-7} / \mathrm{hr}$ to include the failure rate of the digital unit as well $\left(1.10^{-7} / \mathrm{hr}+5 \cdot 10^{-7} / \mathrm{hr}\right)$. The failure rate $\lambda 1$ shown in Figure 6.13 is set to be $7.2 .10^{-7} / \mathrm{hr}$. The coverage factor for the system with DMR and our system is assumed to be $95 \%$, while for the system with TMR it is $99.99 \%$. In general, it is considered that the $2 / 3$ voting results in TMR systems provide nearly a perfect coverage for the first failure. The reliability of the module 
for fault detection and reconfiguration in the system with DMR $\left(R_{d}\right)$ and the voter module in TMR $\left(R_{v}\right)$ is assumed to be 1 (fault-free). This is because we are interested in the reliability performance resulting from the redundancy of the AMR sensor.

Figure 6.14 shows the reliability results of the four systems that have been calculated with the Equations (6.3), (6.10), (6.13) and (6.17) for a period of 1000k hours. In the first 200k hours TMR shows the best values due to the higher coverage factor is used compared with the other systems. However, the TMR performance decreases faster than DMR or our system (AMR+), which is justified by the fact that more modules also means more probabilities of fails. Overall, our system provides the best reliability during the period of time considered while only one AMR sensor shows the lowest reliability values.

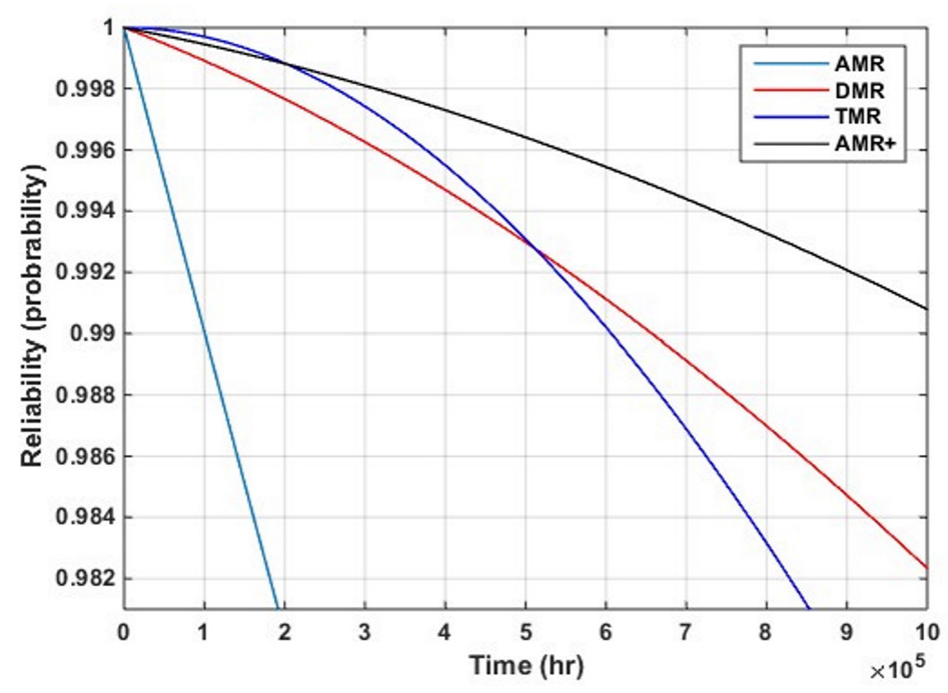

Figure 6.14: Reliability of an AMR sensor, system with DRM, system with TRM and our system $(\mathrm{AMR}+)$ for a period of 1000k hours.

Now, if one takes into consideration the failure rate goal of $1.10^{-9} / \mathrm{hr}$ for automotive applications as well as a demanded mission time of 15 years, this results in a reliability requirement of 0.999 as shown in Equation 6.18.

$$
\text { Reliability }[131400]=e^{-1.10^{-9} \cdot(131400)}=0.999
$$

Figure 6.15 shows the first 200k hours of the results presented in Figure 6.14. Our system and the TMR system show reliability values of 0.992 and 0.994 respectively at $135 \mathrm{k}$ hours, which means both satisfy the reliability requirement. However, for TMR we are not considering the reliability of the voter module, which means that in reality a lower reliability value for the system with TMR can be expected. 


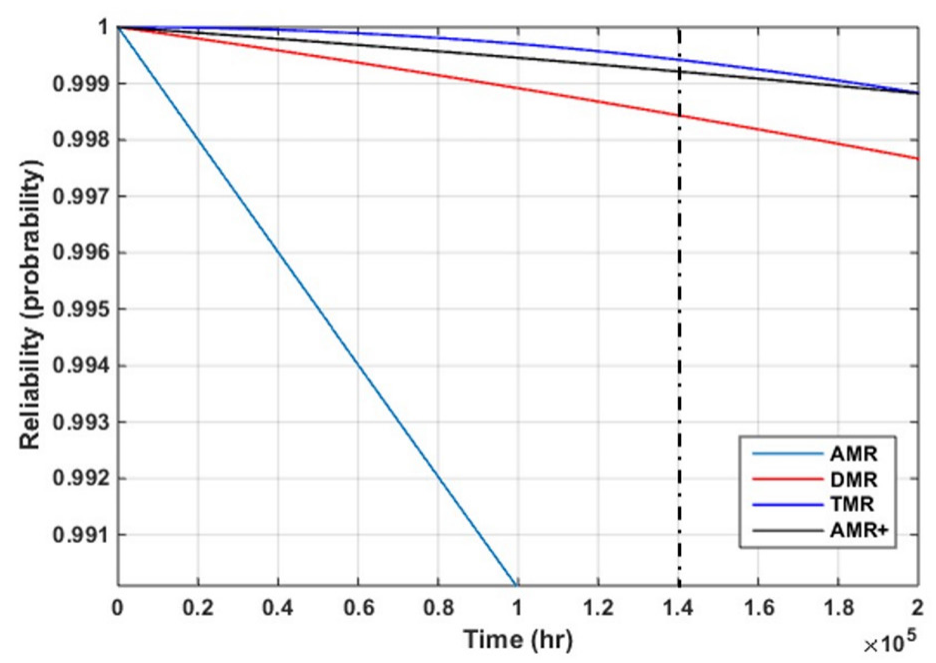

Figure 6.15: Reliability of an AMR sensor, DMR system, TMR system and our system $(\mathrm{AMR}+)$ for a period of $200 \mathrm{k}$ hours.

With respect to safety, the standard ISO 26262 that is focussed on functional safety for electronic systems used in automotive applications specifies four ASIL levels (A to D) linked to maximum failure rates, as presented in chapter 2. Level $\mathrm{D}$ demands the lowest failure rate $\left(<10^{-8}\right)$ and it is usually the target level for safety-critical applications. The failure rate is a metric for reliability and a failure rate of $1.10^{-8} / \mathrm{hr}$ implies a reliability of 0.998 for a mission time of 15 years. From the reliability values shown in Figure 6.15 can be concluded that our system, as well as the system with DMR and TMR satisfy the level D requirement. However, a small failure rate is not the only requirement for safety. So far, automotive sensors should be fail-operational, meaning they should be able to tolerate one catastrophic fault. This is the case for our system and the system with DRM and TMR. However, after tolerating a catastrophic fault it is important that the sensor is replaced within a limited period of time to avoid that it represents a risk for the users of the car.

Regarding the dependability attribute maintainability, a system is considered to be maintainable in case it can continue in operation after being repaired. However, as mentioned in chapter 2, a repair in the traditional sense is not feasible for most modern embedded systems. Instead, maintainability is achieved by methods aimed to guarantee the correct functionality of the system. In our AMR sensor system, the fault-tolerance module allows the sensor to continue its operation despite a catastrophic fault. Additionally, the performance monitoring module is aimed to keep the sensor performance over time despite aging effects. In this way, it is avoided that undesired parameters such as the offset voltage lead to a system malfunction for being out of the allowed range. Although the systems with DMR and TMR are also maintainable, they are usually focussed on catastrophic faults only. Aging compensation is more complicated in these systems as actions are required for each sensor.

In terms of availability, the system downtime is usually of particular interest. However, this parameter is linked to the actual implementation of the fault-tolerant 
systems, and hence it is difficult to provide specific values. In our system, it is proposed to implement the fault-detection and fault-location part of the fault-tolerant system in the analog domain aiming for a short downtime. Although the maximum downtime allowed depends on the application in which the sensor is used, a short downtime is always desired, especially in safety-critical applications.

\subsection{Conclusions}

The dependability requirements of automotive applications are continuously increasing, which implies that the requirements for electronic components used in cars are rising as well. Therefore it is vital to embrace strategies that allow the components to satisfy the requirements demanded of them. In this chapter, we have proposed an architecture for the AMR sensor system, which includes the methods proposed in previous chapters to handle catastrophic faults and to keep the sensor performance over time. The system is capable of compensating in real-time the sensor outputs for the undesired parameters offset voltage and amplitude imbalance, using factors that are updated as required based on monitoring of the angle error. It includes a manager to control all the operations, while the standard IEEE 1687 has been proposed to connect the internal system modules aimed to improve the dependability of the AMR sensor, as well as to connect the AMR sensor system with other clusters that are parts of a network for dependability and/or fault-management implemented in the car.

Regarding the dependability attributes the following remarks can be made. The reliability of our AMR sensor system, as well as an AMR sensor and systems with DMR (Dual Modular Redundancy) and TMR (Triple Modular Redundancy), have been calculated using values for the failure rates and the coverage factors that are often considered for automotive electronic components. The results obtained shows that our system can be considered the most reliable among all. This is because the proposed fault-tolerance method is based on analytical redundancy, so additional sensors are not required unlike systems with DMR or TMR. This also represents an advantage in terms of space and production costs, both sensitive aspects in automotive applications. In terms of safety, our AMR sensor system is fail-operational and results show it can satisfy the ASIL level D, which is usually demanded in safety-critical applications. The maintainability is enhanced by allowing the system to undergo modifications to keep the sensor performance over time, as well as by guaranteeing that the sensor continues its operation despite catastrophic faults.

Compared to our proposed AMR sensor system, a system with TMR can also satisfy the dependability requirements of reliability and safety in automotive applications, but at a higher price in terms of space and manufacturing costs. Regarding maintainability, a system based on physical redundancy will demand more system resources to keep the sensor performance over time. Our proposed system can provide the required failure rate while demanding fewer resources of the system to maintain the sensor performance over time, which is always desirable as the processing resources are limited during the sensor lifetime. 


\section{References}

[ASS11] ASSET. "IEEE P1687 Internal JTAG (IJTAG) Tutorial". 2011.

[Avi04] A. Avizienis, J. C. Laprie, B. Randell, and C. Landwehr. "Basic concepts and taxonomy of dependable and secure computing". IEEE Transactions on Dependable and Secure Computing, volume 1(1), pp. 11-33, Jan 2004.

[Ber13] S. Bernardi, J. Merseguer, and D. Petriu. "Dependability Analysis Techniques". Model-Driven Dependability Assessment of Software Systems, pp. 73-90, 2013.

[Buj04] G. Buja, S. Castellan, R. Menis, and A. Zuccollo. "Dependability of safety-critical systems". In IEEE International Conference on Industrial Technology, IEEE ICIT '04., volume 3, pp. 1561-1566, Dec 2004.

[Fuq03] N. Fuqua. "The applicability of Markov Analysis Methods to Reliability, Maintainability and Safety". Selected topics in assurance related technologies, volume 10(2), p. 6, 2003.

[Ham03] R. Hammett and P. Babcock. "Achieving 10-9 Dependability with Driveby-Wire Systems". SAE Technical Paper 2003-01-1290, pp. 157-170, March 2003.

[Ibr18] A. Ibrahim. Test Standards Reuse for Structured and Cost-Efficient Dependability Management of System-on-Chips. Ph.D. thesis, University of Twente, Enschede, April 2018.

[IEC03] IEC International Electrotechnical Commission. "International standard IEC 60300-3-1 dependability management". 2003.

[IEE14] IEEE. "IEEE Standard for Access and Control of Instrumentation Embedded within a Semiconductor Device". IEEE Std 1687-2014, pp. 1-283, Dec 2014 .

[Ise02] R. Isermann, R. Schwarz, and S. Stolzl. "Fault-tolerant drive-by-wire systems". IEEE Control Systems, volume 22, pp. 64-81, Oct 2002.

[Joh11] M. A. Johar, R. Freier, and A. Koenig. "Adding self-x capabilities to AMR sensors as a first step towards dependable embedded systems". pp. 41-46, July 2011.

[Jut13] A. Jutman, S. Devadze, and K. Shibin. "Effective Scalable IEEE 1687 Instrumentation Network for Fault Management". IEEE Design Test, volume 30(5), pp. 26-35, Oct 2013.

[Ker10] H. G. Kerkhoff and J. Wan. "Dependable digitally-assisted mixed-signal IPs based on integrated self-test amp; self-calibration". In 16th International Mixed-Signals, Sensors and Systems Test Workshop (IMS3TW), pp. 1-6, June 2010. 
[Kha14] M. A. Khan. On improving dependability of analog and mixed-signal SoCs: a system-level approach. Ph.D. thesis, University of Twente, Enschede, Nov 2014.

[Kim02] K. H. Kim, J. Hyung-Joon, and L. Keyseo. "The design and evaluation of all voting triple modular redundancy system". Proceedings of the Annual Reliability and Maintainability Symposium, jan 2002.

[NXP11] NXP. "KMZ49 datasheet". NXP semiconductors, 2011.

[Pen12] J.-J. Peng, Y.-P. Liu, and Y.-Y. Chen. "A dependability model for TMR system". International Journal of Automation and Computing, volume 9(3), pp. 315-324, Jun 2012.

[Pim04] J. Pimentel. "An Architecture for a Safety-Critical Steer-by-Wire System". SAE Technical Paper, March 2004.

[Sch15] W. Scheibenzuber, F. Moissl, and J. Nimler. "Dual-sensor package technology supporting ASIL-D in safety-related automotive applications". In 17th International Conference on Sensors and Measurement Technology, pp. 469-471, May 2015.

[Shi14] K. Shibin, S. Devadze, and A. Jutman. "Asynchronous Fault Detection in IEEE P1687 Instrument Network". 2014 IEEE 23rd North Atlantic Test Workshop, pp. 73-78, May 2014.

[Sin11] P. Sinha. "Architectural design and reliability analysis of a fail-operational brake-by-wire system from ISO 26262 perspectives". Reliability Engineering and System Safety, volume 96, pp. 1349 - 1359, 2011.

[Smi11] D. Smith. "Reliability, Maintainability and Risk". Elsevier, 2011.

[Son16] K. Son, D. Kim, and C.Kim. "Study on the systematic approach of Markov modeling for dependability analysis of complex fault-tolerant features with voting logics". Reliability Engineering 83 System Safety, volume 150, pp. 44 $-57,2016$.

[Tre01] C. Treutler. "Magnetic sensors for automotive applications". Sensors and Actuators A : Physical, volume 91(1-2), pp. 2-6, 2001.

[Zam17a] A. Zambrano and H. G. Kerkhoff. "A dependable AMR sensor system for automotive applications". In International Test Conference in Asia (ITC-Asia), pp. 59-64. Sept 2017.

[Zam17b] A. Zambrano and H. G. Kerkhoff. "Improving the dependability of AMR sensors used in automotive applications". In 22nd IEEE European Test Symposium (ETS), pp. 1-2. May 2017. 


\section{Chapter 7}

\section{Conclusions and Recommendations}

\subsection{Introduction}

The automotive industry is relying more and more on electronic systems to further improve safety, comfort, efficiency as well as the performance of the cars. These systems demand reliable, and low-cost sensors that are capable of operating for a long time in a highly dependable manner; this is especially important for safety-critical applications. This research focussed on Anisotropic Magnetoresistance (AMR) sensors for angle measurements, which are often used in automotive, in applications such as fuel level detection, headlight adjustment and steering control of which the last one is classified as safety-critical. The goal of this thesis was to propose a system that allows AMR sensors for angle measurements to satisfy the continuously increasing dependability and accuracy requirements demanded by automotive applications. In the remainder of this chapter, the thesis contributions are summarized and discussed, the general conclusions are presented, and finally suggestions for future work are given.

\subsection{Contributions}

Each contribution addresses a research question specified in chapter 1 for the development of the proposed system. The original contributions of this thesis are listed as follows.

\subsubsection{Aging effects on AMR sensors for angle measurements}

Although wearing and aging effects impact the performance of AMR sensors, so far it is possible to satisfy the accuracy requirements without the necessity of aging compensation. However, it is expected that this will change in the near future as a better performance over the sensor lifetime will be required. Therefore, in order to have a better understanding of the aging effects on AMR sensors, as to the best of our knowledge there has been little published data on this issue, it was decided to 
perform a set of aging tests as detailed in chapter 3 .

A set of commercial AMR sensors KMZ49 from NXP were submitted to accelerated degradation tests in which the stress conditions were defined based on the expected operating conditions of the sensor. This allows simulating the performance degradation of the sensor in real-life in a more realistic way. The parameters: offset voltage, the amplitude of the sensor outputs, amplitude imbalance between them, bridge resistances and angle errors have been investigated. The results show that the sensor performance drifts over time; being the offset voltage the most significant source of angle error at the start of the sensor's life, but it also shows the largest drift caused by aging effects. The trend of the drift of the offset voltage can be described as higher at the start but then slowing down over time, which is most likely linked to a stress relaxation effect in the sensor. The undesired parameters amplitude imbalance between the sinusoidal signals at the sensor outputs and additional harmonics present smaller and more stable values over time and are hence considered to be a second-order effect concerning the angle error [Zam15a, Zam16a].

\subsubsection{Aging compensation in AMR sensors}

So far, the sensor outputs are mainly compensated for offset voltage using factors calculated at the start of the sensor's life. Although the drift obtained during our aging tests allows complying with the current requirement of an angle accuracy better than $1^{\circ}$ over the sensor lifetime, this will change in the future when an accuracy requirement better than $0.1^{\circ}$ over the sensor lifetime can be expected. Therefore it is important to embrace strategies that allow the sensor to handle its performance degradation over time without disturbing its normal operation. In chapter 4 the addition of self-X properties for this purpose has been proposed.

For self-monitoring, a set of equations aimed to calculate the maximum angle error online, resulting from the undesired parameters offset voltage, amplitude imbalance and additional harmonics have been proposed. Taking into account that the angle error is a critical parameter in the performance of the sensor, it is a suitable parameter to determine whether aging compensation is required. In this case, the self-calibration property allows calculating online the compensation factors for offset voltage and amplitude imbalance. These self-X properties have been defined based on the geometrical characteristics of the sensor outputs. The functionality of the self-monitoring property has been verified in MATLAB using data obtained from an analytical model of the sensor but also with data measured in commercial sensors. In case of self-calibration, an FPGA implementation was additionally included [Zam15c, Zam16c, Zam16b].

\subsubsection{Fault-tolerant system for AMR sensors}

The sensor operation should be guaranteed despite the occurrence of a catastrophic fault. Therefore, chapter 5 presents a fault-tolerant system aimed to keep the AMR sensor in operation despite a catastrophic fault in its bridge resistances or 


\subsection{Conclusions}

its connections to the power supply or ground. A fault condition is handled via fault detection, location, reconfiguration and recovery. Fault-detection and faultlocation are performed by verifying the output voltages of the Wheatstone bridges in the sensor. Fault-reconfiguration in case of a faulty bridge resistance is executed by analytical redundancy based on the characteristics of the output voltages of the non-faulty half-bridges, which can be combined in order to recover the sensor operation. In the event of a broken bridge supply line or broken bridge ground connection, fault-reconfiguration is performed by switching over to a backup power supply that should be available in a dependable system. Our system can tolerate a faulty half-bridge with one faulty resistance (open or short condition) or two faulty resistances (one in open condition and the other in short condition); it is not the same for faulty resistances in two half-bridges, in which case the sensor cannot be longer used. In the event that a faulty half-bridge and a broken bridge connection to the power supply or ground occurs at the same time, the fault-tolerant system first handles the broken connection and then the faulty half-bridge. The system functionality has been verified in MATLAB with data obtained from an analytical model of an AMR sensor but also from commercial sensors [Zam15b].

\subsubsection{Architecture of the proposed AMR sensor system}

Chapter 6 presents the architecture of the proposed AMR sensor system, which includes the self-X properties and the fault-tolerant method defined in chapter 4 and 5 respectively. A manager to handle all the activities of the system has also been included. The IEEE 1687 standard is considered for the connection of the internal modules of the AMR sensor, as well as for the communication with other components that are part of a dependability network implemented in the car. The sensor outputs are compensated in real-time with factors that are only updated when it is required based on the self-X properties. Our fault-tolerance module allows guaranteeing the correct service of the sensor despite the occurrence of a catastrophic fault, which might (with no fault-tolerance measures) in principle causes the sensor to suddenly stop working [Zam17b], [Zam17a].

\subsection{Conclusions}

This thesis focussed on improving the dependability of AMR sensors used for angle measurements. Besides performance degradation, these sensors can also be affected by catastrophic faults that in principle cause the sensor to suddenly stop working. From the results obtained in our aging tests it can be concluded that although the undesired parameters included in the sensor outputs drift over time, the sensor performance remains within the tolerance band currently permitted to guarantee an angle accuracy better than $1^{\circ}$ over the sensor lifetime. However, the tolerance band for drifting will get smaller in the future as an accuracy requirement better than $0.1^{\circ}$ over the sensor lifetime can be expected, especially with the current trend of autonomous cars. Therefore, though until now the sensor outputs are mainly compensated for the offset voltage using factors calculated at the start of the sensor 
life, in the future aging compensation will also be required.

In this research, the addition of self-X properties to allow the AMR sensor to handle the performance degradation over time has been proposed. By self-monitoring of the maximum angle error, it is determined whether aging compensation is required in which case self-calibration allows updating the compensation factors for offset voltage and amplitude imbalance without disturbing the normal operation of the sensor. The self-X properties are executed based on digital signal processing of the sensor outputs. Although the compensation factors cannot be calculated in real-time, this does not represent a problem because in chapter 3 it has been concluded that the undesired parameters in the sensor outputs drift slowly over time. In fact, taking into consideration that processing resources are limited during the sensor lifetime, we suggest it is better to compensate in real-time the sensor outputs but calculate the compensation factors when it is actually required.

Additionally, a fault-tolerant system capable of tolerating faulty bridge resistances as well as broken bridge connections to the power supply or ground in AMR sensors has been proposed. Faulty resistances are handled by usage of analytical redundancy without adding extra sensors as is usually done nowadays. This represents an advantage in terms of physical space and production cost, both critical factors for mass production in the automotive industry. In case of broken bridge connections to the power supply or ground, one can switch-over to a backup power supply that should be included in a dependable system.

A dependability assessment focussed on the attributes reliability, safety, maintainability, and availability has been performed in which the failure rates and the coverage factors were defined based on values often considered for typical automotive electronic components. Besides our AMR sensor system, three other systems with one AMR sensor, two AMR sensors configured in DMR (Dual Modular Redundancy) and three AMR sensors in TMR (Triple Modular Redundancy) have been included for comparison purposes. Based on the results that have been obtained, it can be shown that our system is the most reliable among all, as well as it can fulfil the requirement of a failure rate of $1.10^{-9} / \mathrm{h}$ that is the goal for automotive applications. In terms of safety, it can satisfy the ASIL level D that is usually applied for safety-critical applications and also it is fail-operational as required for automotive sensors. The maintainability is improved by the fault-tolerant system that allows the sensor to continue its operation despite the occurrence of a catastrophic fault as well as by the self-X properties aimed for the sensor to keep its performance over time. Regarding availability, it is proposed to implement the fault-detection and fault-location steps of the fault-tolerant system in the analog domain. The goal is to have a short downtime as this it is always desired.

The results obtained for the system with the sensors configured in TMR show that with this system it is also possible to satisfy the dependability requirements of reliability and safety in automotive applications. However, in terms of maintainability, this kind of system will demand more resources to keep the performance 
over time compared to our system as it is required actions for each sensor. This is especially important with the current trend in automotive of including more and more X-by-wire systems that demand dependable sensors but in which processing resources are limited.

\subsection{Recommendations for future work}

The research work presented in this thesis focussed on improving the dependability of AMR sensors used for angle measurements in automotive applications. An AMR sensor system has been proposed which includes self-X properties as well as a faulttolerant method based on digital signal processing of the sensor outputs. These have been validated in MATLAB using as input, data obtained from an analytical model of the sensor, but also with data collected from commercial sensors. Although the self-calibration property has also been implemented in FPGA, the following step for further validation will be to build a prototype to implement the complete system using either a microcontroller or an FPGA for digital processing. These prototypes can also be used in the validation of an X-by-wire system in which the sensor will be integrated.

Additionally, a further investigation can be made regarding the inclusion of the proposed system in a fault-management architecture. Fault-management is aimed to handle catastrophic faults but also performance degradation. The IEEE 1687 interface allows sending information concerning the sensor fault-condition as well as its performance over time to other components of an X-by-wire system in which the AMR sensor is integrated. Fault-management will become increasingly important in the future, in particular with the current trend of autonomous cars.

Finally, the aging tests that have been executed during our research allow having a better understanding of the aging effects on AMR sensors. This can be the foundation of a new research direction focussed on the development of degradation models of the performance of AMR sensors. 


\section{List of Publications}

[Zam15a] A. Zambrano and H. G. Kerkhoff. "Determination of the aging offset voltage of AMR sensors based on accelerated degradation test". In 20th IEEE International Mixed-Signals Testing Workshop (IMSTW), pp. 1-5, June 2015.

[Zam15b] A. Zambrano and H. G. Kerkhoff. "Fault-tolerant system for catastrophic faults in AMR sensors". In 21st IEEE International On-Line Testing Symposium (IOLTS), pp. 65-70, July 2015.

[Zam15c] A. Zambrano and H. G. Kerkhoff. "Online digital offset voltage compensation method for AMR sensors". In proceedings of IEEE International Instrumentation and Measurement Technology Conference (I2MTC), pp. 1512-1515, May 2015. ISSN 1091-5281.

[Zam16a] A. Zambrano and H. G. Kerkhoff. "Determination of the drift of the maximum angle error in AMR sensors due to aging". In 21st IEEE International Mixed-Signal Testing Workshop (IMSTW), pp. 1-5, July 2016.

[Zam16b] A. Zambrano and H. G. Kerkhoff. "Online digital compensation Method for AMR sensors". In proceedings of IFIP/IEEE International Conference on Very Large Scale Integration (VLSI-SoC), pp. 1-6, Sept 2016.

[Zam16c] A. Zambrano and H. G. Kerkhoff. "Online monitoring of the maximum angle error in AMR sensors". In 22nd IEEE International Symposium on On-Line Testing and Robust System Design (IOLTS), pp. 211-212, July 2016.

[Zam17a] A. Zambrano and H. G. Kerkhoff. "A dependable AMR sensor system for automotive applications". In International Test Conference in Asia (ITC-Asia), pp. 59-64, Sept 2017.

[Zam17b] A. Zambrano and H. G. Kerkhoff. "Improving the dependability of AMR sensors used in automotive applications". In 22nd IEEE European Test Symposium (ETS), pp. 1-2, May 2017. 


\section{Appendix A \\ Virtual Instruments (VI) \\ developed in LabVIEW for the \\ aging tests}




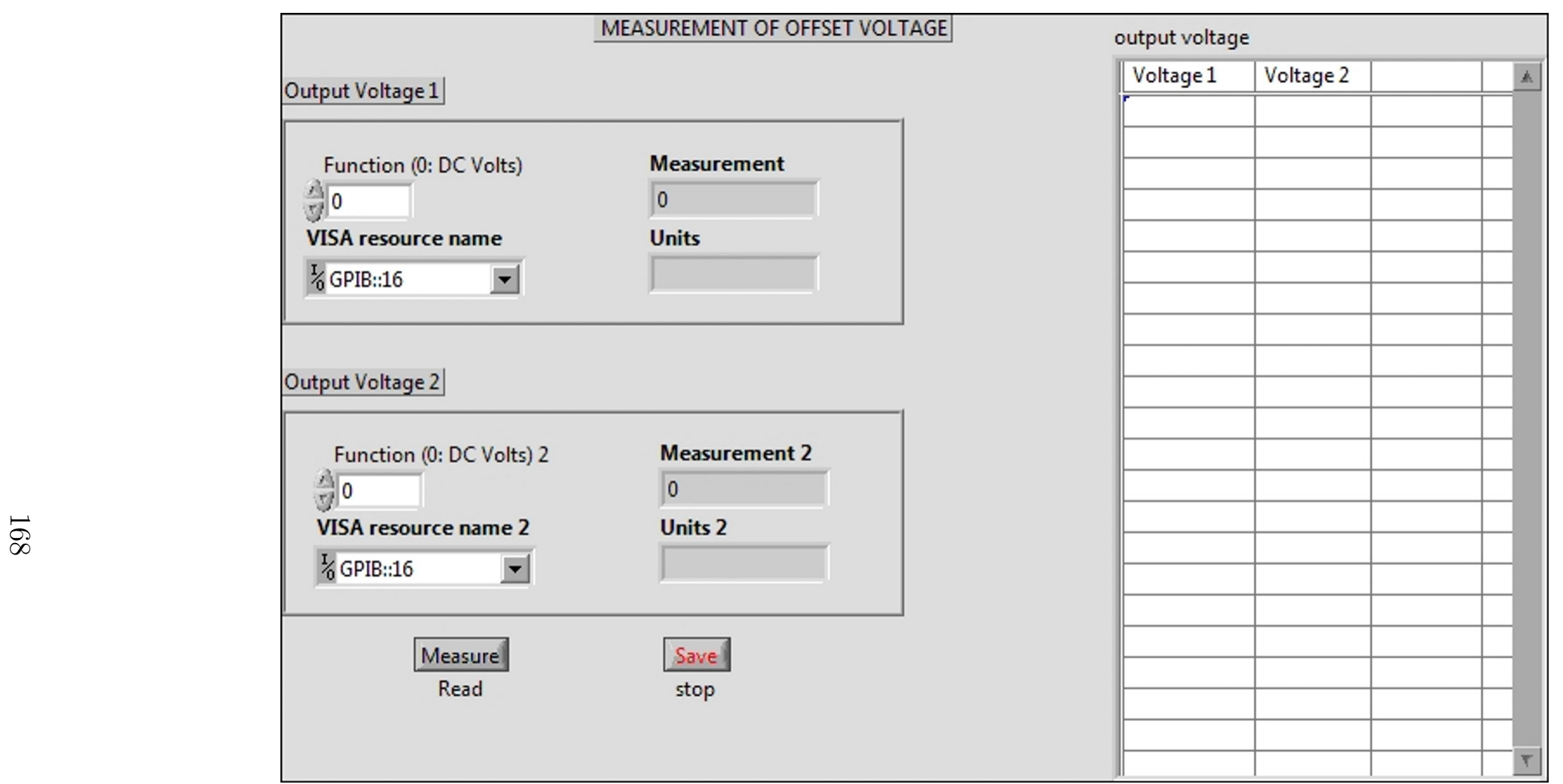

Figure A.1: Front panel of the VI developed in LabVIEW for the first aging test. 


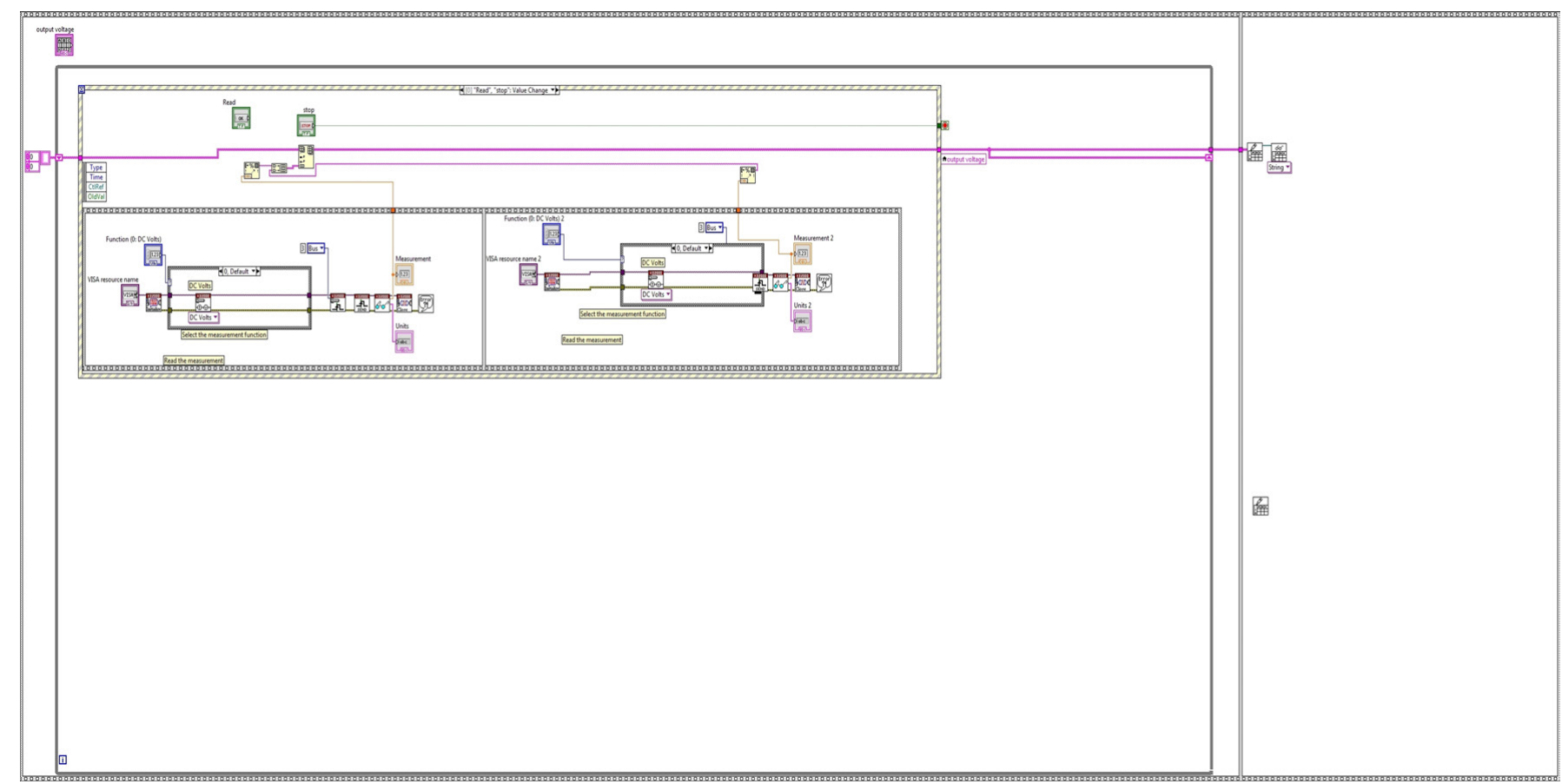

Figure A.2: Part of the block diagram of the VI developed in LabVIEW for the first aging test. 


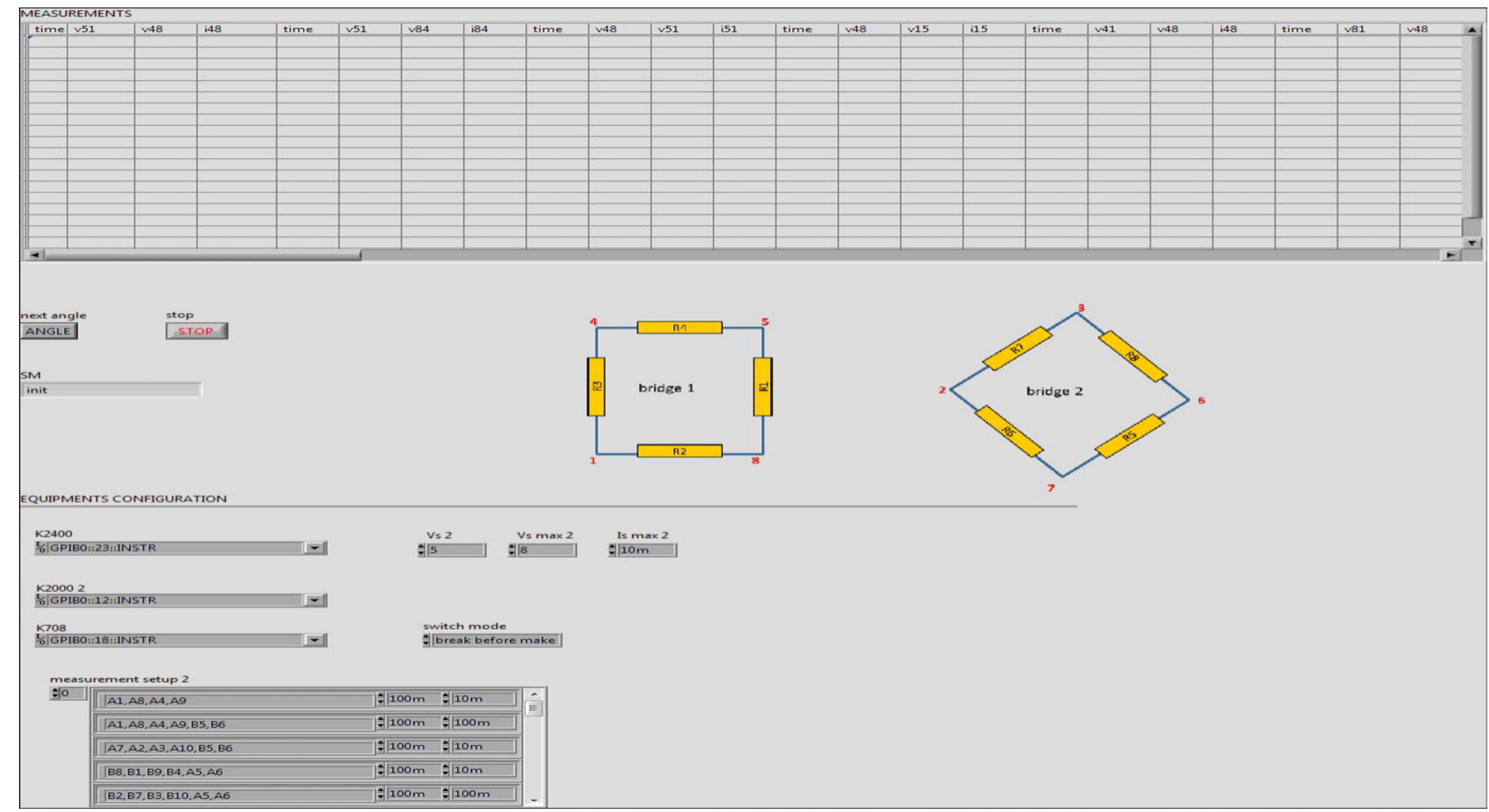

Figure A.3: Front panel of the VI developed in LabVIEW for the second aging test. 


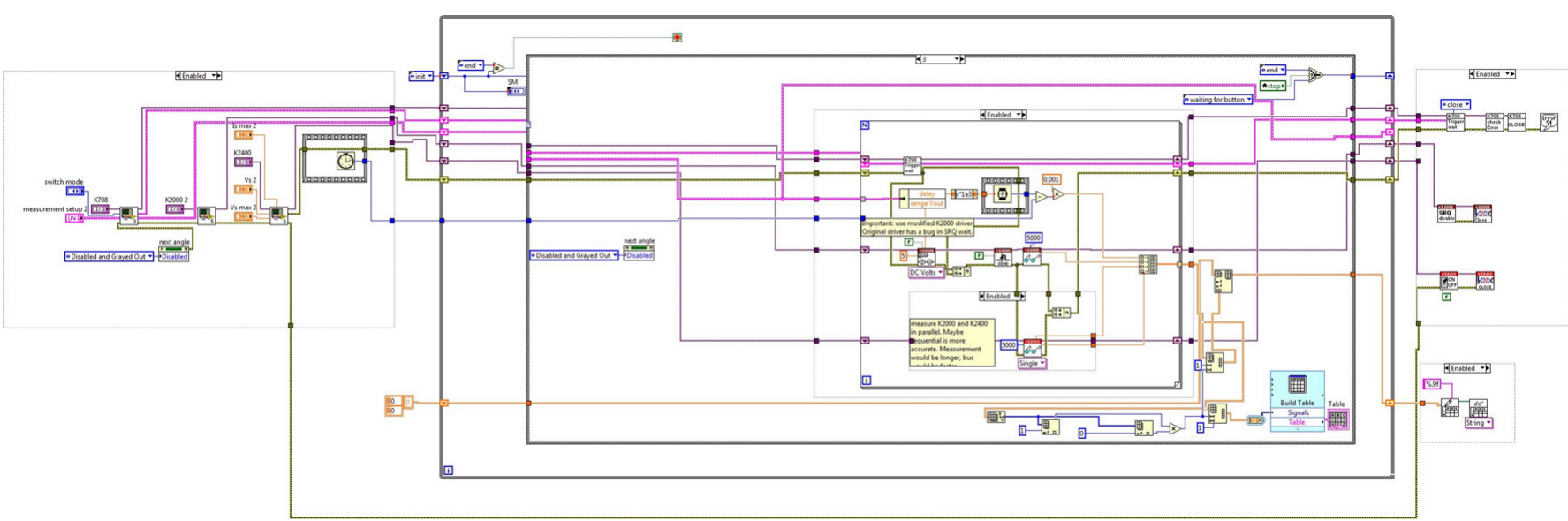

Figure A.4: Part of the block diagram of the VI developed in LabVIEW for the second aging test. 
This page intentionally left blank. 


\section{Appendix B Analytical model of an AMR sensor for angle measurements implemented in MATLAB}




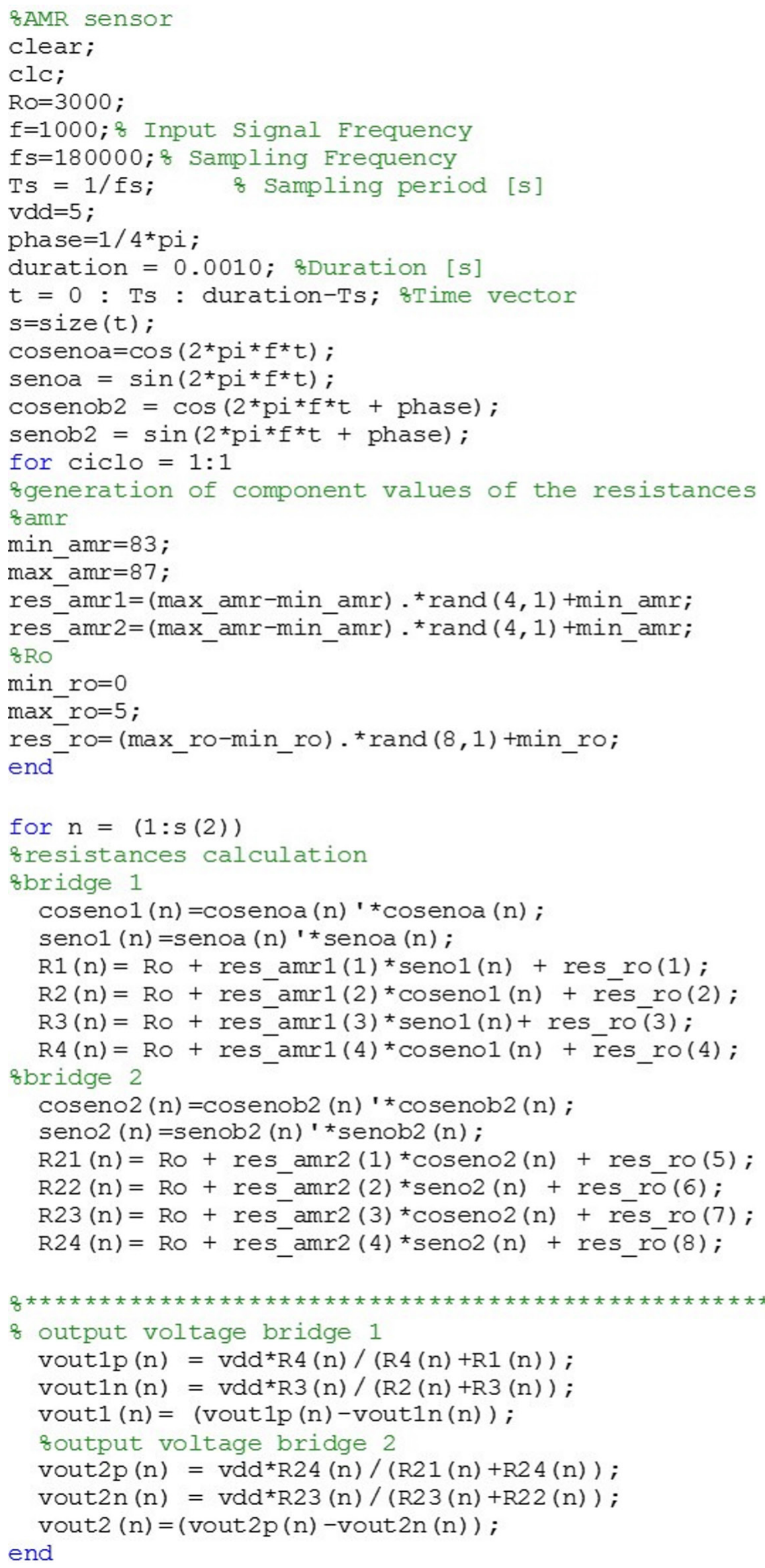




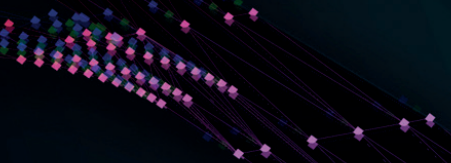

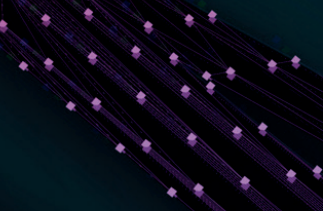

$\therefore 0^{\circ} \quad 0^{\circ} \quad 0^{\circ}, \ldots$
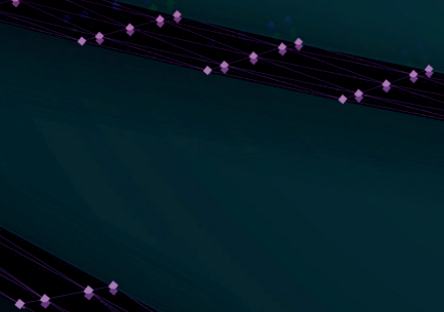

$+\ldots$
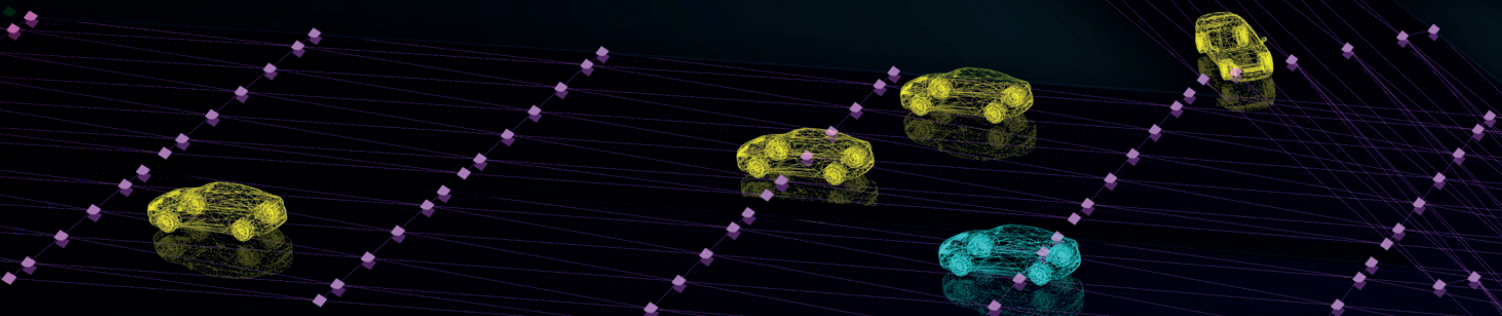

$+5$
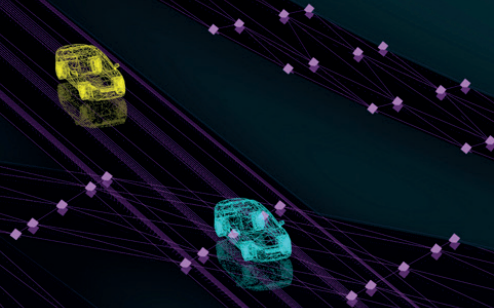

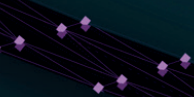

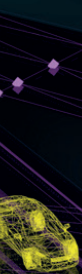

